\title{
The Stabilites of Carbenes: Independent Measures for Singlets and Triplets
}

\author{
Scott Gronert, ${ }^{\dagger}$ James R. Keeffe, ${ }^{\ddagger}$ and Rory A. More O'Ferrall ${ }^{\S}$ \\ ${ }^{\dagger}$ Department of Chemistry, Virginia Commonwealth University, 1001 W. Main St., Richmond, \\ Virginia 23284. "Department of Chemistry and Biochemistry, San Francisco State University, \\ 1600 Holloway Avenue, San Francisco, California 94132. ${ }^{\$}$ School of Chemistry and Chemical \\ Biology, University College Dublin, Belfield, Dublin 4, Ireland.
}

\section{Supporting Information}

$\underline{\text { Table of Contents }}$

1.

2.

3.

4.

5.

6.

3. Frisch, M. J.; Trucks, G. W.; Schlegel, H. B.; Scuseria, G. E.; Robb, M. A.; Cheeseman, J. R.; Montgomery, J. A.; Vreven, T.; Kudin, K. N.; Burant, J. C.; Millam, J. M.; Iyengar, S. S.; Tomasi, J.; Barone, V.; Mennucci, B.; Cossi, M.; Scalmani, G.; Rega, N.; Petersson, G. A.; Nakatsuji, H.; Hada, M.; Ehara, M.; Toyota, K.; Fukuda, R.; Hasegawa, J.; Ishida, M.; Nakajima, T.; Honda, Y.; Kitao, O.; Nakai, H.; Klene, M.; Li, X.; Knox, J. E.; Hratchian, H. P.; Cross, J. B.; Adamo, C.; Jaramillo, J.; Gomperts, R.; Stratmann, R. E.; Yazyev, O.; Austin, A. J.; Cammi, R.; Pomelli, C.; Ochterski, J. W.; Ayala, P. Y.; Morokuma, K.; Voth, G. A.; Salvador, P.; Dannenberg, J. J.; Zakrzewski, V. G.; Dapprich, S.; Daniels, A. D.; Strain, M. C.; Farkas, O.; Malick, D. K.; Rabuck, A. D.; Raghavachari, K.; Foresman, J. B.; Ortiz, J. V.; Cui, Q.; Baboul, A. G.; Clifford, S.; Cioslowski, J.; Stefanov, B. B.; Liu, G.; Liashenko, A.; Piskorz, P.; Komaromi, I.; Martin, R. L.; Fox, D. J.; Keith, T.; Al-Laham, M. A.; Peng, C. Y.; Nanayakkara, A.; Challacombe, M.; Gill, P. M. W.; Johnson, B.; Chen, W.; Wong, M. W.; Gonzalez, C.; Pople, J. A. Gaussian 03, Revision B04; Gaussian, Inc.: Pittsburgh, PA, 2003. 
Table S1. G3MP2 enthalpies and free energies of carbocations and related species (hartrees). ${ }^{a}$ Point groups ${ }^{\mathrm{b}}$ and selected geometric data

\begin{tabular}{|c|c|c|c|}
\hline Carbocation and Precursors (Point Group) & $\mathrm{H}(298 \mathrm{~K}, 1 \mathrm{~atm} .)^{\mathrm{c}}$ & $\mathrm{G}(298 \mathrm{~K}, 1 \mathrm{~atm} .)^{\mathrm{c}}$ & Geometric Feature $(s)^{\mathrm{d}}$ \\
\hline $\begin{array}{l}\text { water }\left(\mathrm{C}_{2 \mathrm{v}}\right) \\
\text { dihydrogen } \\
\text { hydride }^{-}\end{array}$ & $\begin{array}{l}-76.338150 \\
-1.166608 \\
333.1 \mathrm{kcal} / \mathrm{mol}^{\mathrm{e}}\end{array}$ & $\begin{array}{l}-76.359525 \\
-1.181367\end{array}$ & $\begin{array}{l}d=0.967, \Phi=104.0 \\
d=0.738\end{array}$ \\
\hline $\begin{array}{l}\text { methyl }{ }^{+}\left(\mathrm{D}_{3 \mathrm{~h}}\right) \\
\text { methanol }\left(\mathrm{C}_{\mathrm{s}}\right) \\
{ }^{1} \mathrm{CH}_{2}\left(\mathrm{C}_{2 \mathrm{v}}\right) \\
{ }^{3} \mathrm{CH}_{2}\left(\mathrm{C}_{2 \mathrm{v}}\right)\end{array}$ & $\begin{array}{l}-39.389408 \\
-115.546805 \\
-39.062446 \\
-39.077421\end{array}$ & $\begin{array}{l}-39.410565 \\
-115.573786 \\
-39.083873 \\
-39.099613\end{array}$ & $\begin{array}{l}d(C H)=1.088 \\
d=1.109, \Phi=102.1^{\mathrm{f}} \\
d=1.077, \Phi=131.6^{\mathrm{f}}\end{array}$ \\
\hline $\begin{array}{l}\text { ethyl }{ }^{+}\left(C_{2 v}\right)^{g} \\
\text { ethane }\left(D_{3 d}\right) \\
{ }^{1} \text { methylcarbene }\left(C_{s}\right) \\
{ }^{3} \text { methylcarbene }\left(C_{s}\right)\end{array}$ & $\begin{array}{l}-78.685172 \\
-79.645106 \\
-78.309638 \\
-78.314313\end{array}$ & $\begin{array}{l}-78.712034 \\
-79.670991 \\
-78.335971 \\
-78.342715\end{array}$ & $\begin{array}{l}\Phi=104.4 \\
\Phi=131.5\end{array}$ \\
\hline $\begin{array}{l}\text { allyl }{ }^{+}\left(\mathrm{C}_{2 \mathrm{v}}\right) \\
\text { propene }\left(\mathrm{C}_{1} / \mathrm{C}_{\mathrm{s}}\right) \\
{ }_{\text {vinylcarbene }}\left(\mathrm{C}_{1}\right) \\
{ }^{3} \text { vinylcarbene }\left(\mathrm{C}_{1} / \mathrm{C}_{\mathrm{s}}\right)\end{array}$ & $\begin{array}{l}-116.726685 \\
-117.665968 \\
-116.339787 \\
-116.352932\end{array}$ & $\begin{array}{l}-116.755305 \\
-117.696070 \\
-116.369397 \\
-116.383133\end{array}$ & $\begin{array}{l}\Phi(C C C)=117.6, d(C 1 C 3)=2.365 \\
d(C=C)=1.337 \\
d(C=C)=1.363 \\
d(C=C)=1.369\end{array}$ \\
\hline $\begin{array}{l}\text { propargyl }{ }^{+}\left(\mathrm{C}_{2 \mathrm{v}}\right) \\
\text { propyne }\left(\left(\mathrm{C}_{3 \mathrm{v}}\right)\right. \\
{ }^{1} \text { ethynylcarbene }\left(\mathrm{C}_{1} / \mathrm{C}_{\mathrm{s}}\right) \\
{ }^{3} \text { ethynylcarbene }\left(\mathrm{C}_{1} / \mathrm{C}_{2}\right)\end{array}$ & $\begin{array}{l}-115.472843 \\
-116.438306 \\
-115.118567 \\
-115.136804\end{array}$ & $\begin{array}{l}-115.500946 \\
-116.466220 \\
-115.146896 \\
-115.166782\end{array}$ & $\begin{array}{l}\mathrm{d}(\mathrm{C} \equiv \mathrm{C})=1.232 \\
\mathrm{~d}(\mathrm{C} \equiv \mathrm{C})=1.218 \\
\mathrm{~d}(\mathrm{C} \equiv \mathrm{C})=1.237 \\
\mathrm{~d}(\mathrm{C} \equiv \mathrm{C})=1.266\end{array}$ \\
\hline
\end{tabular}


Table S1. G3MP2 enthalpies and free energies of carbocations and related species (hartrees). ${ }^{a}$ Point groups $^{\mathrm{b}}$ and selected geometric data.

\begin{tabular}{|c|c|c|c|}
\hline Carbocation and Precursors (Point Group) & $\mathrm{H}(298 \mathrm{~K}, 1 \mathrm{~atm} .)^{\mathrm{c}}$ & $\mathrm{G}(298 \mathrm{~K}, 1 \mathrm{~atm} .)^{\mathrm{c}}$ & Geometric Feature $(\mathrm{s})^{\mathrm{d}}$ \\
\hline diethynylCH ${ }^{+}\left(\mathrm{C}_{2 \mathrm{v}}\right)$ & -191.522795 & -191.556352 & $\mathrm{~d}(\mathrm{C}-\mathrm{C})=1.229,1.379, \Phi=122.6$ \\
\hline diethynylmethane $\left(\mathrm{C}_{2 \mathrm{v}}\right)$ & -192.455962 & -192.489501 & $\mathrm{~d}(\mathrm{C}-\mathrm{C})=1.219,1.466, \Phi=112.6$ \\
\hline carbene, singlet $\left(\mathrm{C}_{2 \mathrm{v}}\right)$ & -191.164154 & -191.197553 & $\mathrm{~d}(\mathrm{C}-\mathrm{C})=1.233,1.375, \Phi=120.7$ \\
\hline carbene, triplet $\left(\mathrm{C}_{2 \mathrm{v}} / \mathrm{D}_{\infty \mathrm{h}}\right)$ & -191.184226 & -191.214080 & $\mathrm{~d}(\mathrm{C}-\mathrm{C})=1.219,1.315, \Phi=179.9$ \\
\hline cyclopropyl $^{+}\left(\mathrm{C}_{2 \mathrm{v}}\right)^{\mathrm{h}}$ & -116.671950 & -116.700693 & $\mathrm{~d}(\mathrm{C}-\mathrm{C})=1.529,1.423,1.423$ \\
\hline cyclopropane $\left(\mathrm{D}_{3 \mathrm{~h}}\right)$ & -117.651296 & -117.678193 & $\mathrm{~d}(\mathrm{C}-\mathrm{C})=1.501$ \\
\hline${ }^{1}$ cyclopropylidene $\left(\mathrm{C}_{2 \mathrm{v}}\right)$ & -116.329949 & -116.357737 & $\mathrm{~d}\left(\mathrm{CH}_{2}-\mathrm{CH}_{2}\right)=1.482$ \\
\hline${ }^{3}$ cyclopropylidene $\left(\mathrm{C}_{2 \mathrm{v}}\right)$ & -116.303364 & -116.331868 & $\mathrm{~d}\left(\mathrm{CH}_{2}-\mathrm{CH}_{2}\right)=1.595$ \\
\hline cyclopropylcarbinyl $^{+}$, bisected $\left(\mathrm{C}_{1} / \mathrm{C}_{\mathrm{s}}\right)$ & -155.971663 & -156.004676 & $\mathrm{~d}\left(\mathrm{CH}-\mathrm{CH}_{2}\right)=1.646$ \\
\hline methylcyclopropane $\left(\mathrm{C}_{1} / \mathrm{C}_{\mathrm{s}}\right)$ & -156.886817 & -156.918983 & $\mathrm{~d}\left(\mathrm{CH}-\mathrm{CH}_{2}\right)=1.502$ \\
\hline${ }^{1}$ cyclopropylcarbene, anti $\left(\mathrm{C}_{1} / \mathrm{C}_{\mathrm{s}}\right)$ & -155.568634 & -155.600468 & $\mathrm{~d}\left(\mathrm{CH}-\mathrm{CH}_{2}\right)=1.566$ \\
\hline${ }^{3}$ cyclopropylcarbene $\left(\mathrm{C}_{1}\right)$ & -155.558226 & -155.591165 & $\mathrm{~d}\left(\mathrm{CH}-\mathrm{CH}_{2}\right)=1.513$ \\
\hline cyclobutyl $^{+}\left(\mathrm{C}_{1} / \mathrm{C}_{\mathrm{s}}\right)$ & -155.973935 & -156.006101 & $\mathrm{~d}(\mathrm{C} 1-\mathrm{C} 3)=1.649$ \\
\hline cyclobutane $\left(\mathrm{D}_{2 \mathrm{~d}}\right)$ & -156.886113 & -156.916095 & $\mathrm{~d}(\mathrm{C} 1-\mathrm{C} 3)=2.14, \Theta(\mathrm{CCCC})=21.7$ \\
\hline${ }^{1}$ cyclobutylidene $\left(\mathrm{C}_{1} / \mathrm{C}_{\mathrm{s}}\right)$ & -155.573496 & -155.604680 & $\mathrm{~d}(\mathrm{C} 1-\mathrm{C} 3)=1.711$ \\
\hline${ }^{3}$ cyclobutylidene $\left(\mathrm{C}_{1} / \mathrm{C}_{2 c}\right)$ & -155.556857 & -155.589091 & $\mathrm{~d}(\mathrm{C} 1-\mathrm{C} 3)=2.06$, flat ring \\
\hline isobutyl $^{+}$ & NA & NA & rearranges to $t$-butyl ${ }^{+}$ \\
\hline isobutane $\left(\mathrm{C}_{3 \mathrm{v}}\right)$ & -158.112675 & -158.146020 & $\mathrm{~d}(\mathrm{C}-\mathrm{C})=1.526$ \\
\hline${ }^{1}$ 2-methylpropylidene $\left(\mathrm{C}_{1}\right)$ & -156.778872 & -156.813984 & $\begin{array}{l}\mathrm{d}(\mathrm{C} 1-\mathrm{C} 2)=1.455, \mathrm{~d}\left(\mathrm{C} 2-\mathrm{CH}_{3}\right)= \\
1.515,1.577, \Phi(\mathrm{C} 1 \mathrm{CC})=80.5,117.7\end{array}$ \\
\hline${ }^{3}$ 2-methylpropylidene $\left(\mathrm{C}_{1}\right)$ & -156.779857 & -156.815193 & $\mathrm{~d}(\mathrm{C} 1-\mathrm{C} 2)=1.481, \Phi(\mathrm{HC} 1 \mathrm{C} 2)=131.5$ \\
\hline cyclopentanyl $^{+}\left(\mathrm{C}_{1} / \mathrm{C}_{2}\right)$ & -195.230433 & -195.264036 & $\mathrm{~d}(\mathrm{C} 2-\mathrm{H})=1.094,1.126$ \\
\hline cyclopentane $\left(\mathrm{C}_{2}\right)$ & -196.149476 & -196.185264 & $\mathrm{~d}(\mathrm{C}-\mathrm{H})=1.094-1.097$ \\
\hline
\end{tabular}



${ }^{1}$ cyclopentylidene $\left(\mathrm{C}_{1} / \mathrm{C}_{2}\right)$
$-194.838704$
$-194.872042$
$\mathrm{d}(\mathrm{C} 2-\mathrm{H})=1.094,1.113$
${ }^{3}$ cyclopentylidene $\left(\mathrm{C}_{1} / \mathrm{C}_{2}\right)$
$-194.822892$
$-194.857226$
$\mathrm{d}(\mathrm{C} 2-\mathrm{H})=1.094,1.100$

Table S1. G3MP2 enthalpies and free energies of carbocations and related species (hartrees). ${ }^{a}$ Point groups $^{\mathrm{b}}$ and selected geometric data.

\begin{tabular}{|c|c|c|c|}
\hline Carbocation and Precursors (Point Group) & $\mathrm{H}(298 \mathrm{~K}, 1 \mathrm{~atm} .)^{\mathrm{c}}$ & $\mathrm{G}(298 \mathrm{~K}, 1 \mathrm{~atm} .)^{\mathrm{c}}$ & Geometric Feature(s) ${ }^{\mathrm{d}}$ \\
\hline cyclohexadienyl $^{=}\left(\mathrm{C}_{2 \mathrm{v}}\right)$ & -232.103032 & -232.136479 & $\mathrm{~d}(\mathrm{HC}-\mathrm{H})=1.109, \Phi(\mathrm{HCH})=100.6$ \\
\hline cyclohexa-1,3-diene $\left(\mathrm{C}_{2}\right)$ & -232.976477 & -233.011170 & $\mathrm{~d}(\mathrm{C}=\mathrm{C}) 1.353$ \\
\hline cyclohexa-1,4-diene $\left(\mathrm{D}_{2 \mathrm{~h}}\right)$ & -232.976695 & -232.010494 & $\mathrm{~d}(\mathrm{C}=\mathrm{C}) 1.343$ \\
\hline${ }^{1}$ cyclohexa-2,4-dienylidene $\left(\mathrm{C}_{1} / \mathrm{C}_{\mathrm{s}}\right)$ & -231.677009 & -231.710628 & $\mathrm{~d}(\mathrm{C}=\mathrm{C})=1.382,1.357$ \\
\hline${ }^{3}$ cyclohexa-2,4-dienylidene $\left(\mathrm{C}_{1} / \mathrm{C}_{\mathrm{s}}\right)$ & -231.680697 & -231.715976 & $\mathrm{~d}(\mathrm{C}=\mathrm{C})=1.409,1.341$ \\
\hline${ }^{1}$ cyclohexa-2,5-dienylidene $\left(\mathrm{C}_{2 \mathrm{v}}\right)$ & -231.673546 & -2231.707632 & $\mathrm{~d}(\mathrm{C}=\mathrm{C})=1.363$ \\
\hline 6,6-dimethylcyclohexa-2,4-dienyl ${ }^{+}\left(\mathrm{C}_{2 \mathrm{v}}\right)$ & -310.577328 & -310.617208 & $\mathrm{~d}(\mathrm{C}-\mathrm{Me})=1.569, \Phi(\mathrm{MeCMe})=107.3$ \\
\hline 6,6-dimethyl-1,3-cyclohexadiene $\left(\mathrm{C}_{1}\right)$ & -311.450117 & -311.490462 & $\mathrm{~d}(\mathrm{C}-\mathrm{Me})=1.527,1.535$ \\
\hline${ }^{1} 6,6$-dimethylcyclohexa-2,4-dien-1-ylidene $\left(C_{s}\right)$ & -310.144203 & -310.185296 & $\mathrm{~d}(\mathrm{C}-\mathrm{Me})=1.550$ \\
\hline${ }^{3} 6,6$-dimethylcyclohexa-2,4-dien-1-ylidene $\left(C_{s}\right)$ & -310.152664 & -310.194298 & $\mathrm{~d}(\mathrm{C}-\mathrm{Me})=1.539$ \\
\hline phenonium $^{+\mathrm{i}}\left(\mathrm{C}_{\mathrm{s}} / \mathrm{C}_{2 \mathrm{v}}\right)$ & -309.383355 & -309.421655 & $\mathrm{~d}(\mathrm{C} 1-\mathrm{C} 3=\mathrm{d}(\mathrm{C} 2-\mathrm{C} 3)=1.625$ \\
\hline spiro[2.5]octa-4,6-diene $\left(\mathrm{C}_{1}\right)$ & -310.225925 & -310.264568 & $\mathrm{~d}(\mathrm{C} 1-\mathrm{C} 3)=1.504, \mathrm{~d}(\mathrm{C} 2-\mathrm{C} 3)=1.516$ \\
\hline${ }^{1}$ spiro[2.5] octa-4,6-dien-8-ylidene $\left(\mathrm{C}_{\mathrm{s}}\right)$ & -308.945403 & -308.983587 & $\mathrm{~d}(\mathrm{C} 1-\mathrm{C} 3)=\mathrm{d}(\mathrm{C} 2-\mathrm{C} 3)=1.594$ \\
\hline${ }^{3}$ spiro[2.5] octa-4,6-dien-8-ylidene $\left(\mathrm{C}_{\mathrm{s}}\right)$ & -308.933579 & -308.973014 & $\mathrm{~d}(\mathrm{C} 1-\mathrm{C} 3)=\mathrm{d}(\mathrm{C} 2-\mathrm{C} 3)=1.527$ \\
\hline spiro[2.5] octa-4,7-diene $\left(\mathrm{C}_{1} \mathrm{C}_{2 \mathrm{v}}\right)$ & -310.227307 & -310.266052 & $\mathrm{~d}(\mathrm{C} 1-\mathrm{C} 3)=\mathrm{d}(\mathrm{C} 2-\mathrm{C} 3)=1.517$ \\
\hline${ }^{1}$ spiro[2.5] octa-4,7-dien-6-ylidene $\left(\mathrm{C}_{2 \mathrm{v}}\right)$ & -308.931445 & -308.969189 & $\mathrm{~d}(\mathrm{C} 1-\mathrm{C} 3)=\mathrm{d}(\mathrm{C} 2-\mathrm{C} 3)=1.555$ \\
\hline${ }^{3}$ spiro[2.5] octa-4,7-dien-6-ylidene $\left(\mathrm{C}_{2 \mathrm{v}}\right)$ & -308.932797 & -308.971599 & $\mathrm{~d}(\mathrm{C} 1-\mathrm{C} 3)=\mathrm{d}(\mathrm{C} 2-\mathrm{C} 3)=1.528$ \\
\hline
\end{tabular}

${ }^{\mathrm{a} O n e}$ hartree $=627.51 \mathrm{kcal} / \mathrm{mol}$. GAUSSIAN 03, Revision B.04, Frisch, M. J, et al., Gaussian, Inc., Pittsburgh PA, 2003. The G3 method uses a composite "...recipe involving a variety of different models with the purpose of providing accurate themochemical data." See Hehre, W. J. A Guide to Molecular Mechanics and Quantum Chemical Calculations; Wavefunction, Inc.: Irvine, CA, 2003, p 252. Results are estimated to be accurate within about $2 \mathrm{kcal} / \mathrm{mol}$. ${ }^{\mathrm{b}} \mathrm{A}$ slash indicates the species has virtually the higher symmetry. ${ }^{\mathrm{c}} \mathrm{A}$ scaling factor of 0.9135 was used for vibrations. ${ }^{\mathrm{d}}$ Distances $(\mathrm{d})$ are in $\AA$; angles $(\Phi)$ and dihedrals $(\Theta)$ are in degrees. ${ }^{\mathrm{e}}$ This is the experimental electronic energy of the hydride ion. ${ }^{\mathrm{f}}$ The experimental values for the angles are $\sim 102^{\circ}$ for the singlet and $\sim 137^{\circ}$ for the triplet. See (a) Hoffmann, R.; Zeiss, G. D.; Van Dine, G. W. J. Am. Chem. Soc. 1968, 90, 1485. (b) Shavitt, I. Tetrahedron 1985, 41, 1531. Also See Moss, R. A.; .Platz, 
M. S.; Jones, M., Jr., Eds., Reactive Intermediate Chemistry; Wiley-Interscience: New York, 2004, p 277. This volume has five chapters covering various aspects of carbene chemistry. ${ }^{\mathrm{g}}$ The ethyl cation is a symmetrical hydrogen-bridged structure. Some of the data listed here were reported by Keeffe, J. R.; More O'Ferrall, R. A. ARKIVOC 2008, 183, and in Gronert, S.; Keeffe, J. R.; More O'Ferrall, R. A. J. Org. Chem. 2009, 74, 5250. h Cyclopropyl+ is calculated to be a stable speicies at HF/6-31G*. At HF/6-311+G** it is a transition state, iv $=284$ $\mathrm{cm}^{-1}$. At MP2/6-31G* and at MP2/6-311+G** it is also a transition state, $\mathrm{iv}=543 \mathrm{~cm}^{-1}$, shown by an irc calculation in the latter case to

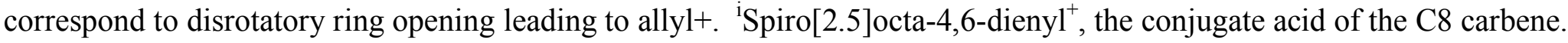


Table S2. Calculated G3MP2 enthalpies and free energies for singlet and triplet substituted arylcarbenes, benzylic cations, and toluenes, 298K.

Substituent (point group)

Enthalpy

$\underline{\text { meta-substituents }}$

$\mathrm{NH}_{2}{ }^{\mathrm{d}}$

singlet carbene $(\mathrm{C} 1)$

triplet carbene $(\mathrm{C} 1)$

cation $(\mathrm{C} 1)$

toluene $(\mathrm{C} 1)$

$\mathrm{OH}$

singlet carbene, syn (Cs)

triplet carbene, syn $(\mathrm{Cs})$

cation, syn (Cs)

toluene, syn $(\mathrm{C} 1)$

$\mathrm{SH}$

singlet carbene, syn $(\mathrm{C} 1 / \mathrm{Cs})$

triplet carbene, gauche $(\mathrm{C} 1)$

cation, anti (C1/Cs)

toluene, gauche $(\mathrm{C} 1)$

$\mathrm{CH}_{3}$

singlet carbene (Cs)

triplet carbene (Cs)

cation (Cs)

toluene $(\mathrm{Cs})$

$\mathrm{H}$

singlet carbene $(\mathrm{C} 1 / \mathrm{Cs})$

triplet carbene $(\mathrm{Cs})$

cation $(\mathrm{C} 1 / \mathrm{C} 2 \mathrm{v})$

toluene (Cs)

$\mathrm{Cl}$

singlet carbene (Cs)

triplet carbene $(\mathrm{Cs})$

cation (Cs)

toluene $(\mathrm{C} 1)$
$-325.016804$

$-325.019243$

$-325.441365$

$-326.341067$

$-344.883001$

$-344.885711$

$-345.297740$

$-346.207573$

$-667.491099$

$-667.493694$

$-667.904223$

$-668.816601$

$-308.971987$

$-308.974587$

$-309.390434$

$-310.297361$

$-269.733859$

$-269.737248$

$-270.148084$

$-271.058922$

$-728.893955$

$-728.897886$

$-729.301667$

$-730.221177$
Free Energy

$-325.055682$

$-325.059673$

$-325,480079$

$-326.382783$

$-344 / 921265$

$-344.925546$

$-345.336303$

$-346.248307$

$-667.531105$

$-667.536125$

$-667.944852$

$-668.860424$

$-309.009895$

$-309.014026$

$-309.428700$

$-310.335106$

$-269.769278$

$-269.774168$

$-270.183853$

$-271.097378$

$-728.932818$

$-728.938297$

$-729.340830$

$-730.263382$ 
Table S2. Calculated G3MP2 enthalpies and free energies for singlet and triplet substituted arylcarbenes, benzylic cations, and toluenes, 298K. ${ }^{\text {a }}$

Substituent (point group)

F

singlet carbene (Cs)

triplet carbene $(\mathrm{Cs})$

cation (Cs)

toluene $(\mathrm{C} 1)$

$\mathrm{CHO}$

singlet carbene $(\mathrm{C} 1 / \mathrm{Cs})$

triplet carbene $\mathrm{C} 1 / \mathrm{Cs}$ )

cation (Cs)

toluene $(\mathrm{C} 1)$
Enthalpy

$-368.893713$

$-368.898044$

$-369.300176$

$-370.220844$

$-382.920172$

$-382.923739$

$-383.324922$

$-384.247069$

$-606.474676$

$-606.479487$

$-606.877086$

$-607.802891$

$-361.859893$

$-361.864759$

$-362.256674$

$-363.189248$ toluene $(\mathrm{C} 1)$

singlet carbene (C1Cs)

triplet carbene (C1Cs)

cation (C1Cs)

$\mathrm{NO}_{2}$

singlet carbene $(\mathrm{C} 1 / \mathrm{Cs})$

triplet carbene $(\mathrm{Cs})$

cation $(\mathrm{C} 1 / \mathrm{Cs})$

toluene $(\mathrm{C} 1)$
$-474.021024$

$-474.026409$

$-474.417238$

$-475.350737$

para-substituents

$\mathrm{NH}_{2}{ }^{\mathrm{d}}$

singlet carbene (C1)

triplet carbene $(\mathrm{C} 1)$

cation $(\mathrm{C} 1 / \mathrm{C} 2 \mathrm{v})$

toluene $(\mathrm{C} 1 / \mathrm{Cs})$
$-325.026133$

$-325.019682$

$-325.473764$

$-326.340208$
Free Energy

$-368.931277$

$-368.937103$

$-369.338025$

$-370.261652$

$-382.960662$

$=382.965726$

$-383.365857$

$-384.291173$

$-606.521184$

$-606.527324$

$-606.922894$

$-607.852198$

$-361.899855$

$-361.906302$

$-362.297040$

$-363.232025$

$-474.063302$

$-474.070345$

$-474.459727$

$-475.395962$
$-325.064051$

$-325.060163$

$-325.512243$

$-326.382712$ 
Table S2. Calculated G3MP2 enthalpies and free energies for singlet and triplet substituted arylcarbenes, benzylic cations, and toluenes, 298K. ${ }^{\mathrm{a}}$

Substituent (point group)

$\mathrm{OH}$

singlet carbene, anti $(\mathrm{C} 1 / \mathrm{Cs})$

triplet carbene, anti $(\mathrm{Cs})$

cation (Cs)

toluene $(\mathrm{C} 1)$

$\mathrm{SH}$

singlet carbene, anti (C1/Cs)

triplet carbene, gauche $(\mathrm{C} 1)$

cation $(\mathrm{C} 1 / \mathrm{Cs})$

toluene, gauche $(\mathrm{C} 1)$

$\mathrm{CH}_{3}$

singlet carbene $(\mathrm{C} 1)$

triplet carbene $((\mathrm{C} 1)$

cation $(\mathrm{C} 1 / \mathrm{Cs})$

toluene $(\mathrm{C} 1 / \mathrm{C} 2 \mathrm{v})$

$\mathrm{CH}_{2} \mathrm{~F}$

singlet carbene, syn $(\mathrm{C} 1 / \mathrm{Cs})$

triplet carbene, gauche $(\mathrm{C} 1)$

cation, syn (Cs)

toluene, gauche $(\mathrm{C} 1)$

$\mathrm{H}$

singlet carbene $(\mathrm{C} 1 / \mathrm{Cs})$

triplet carbene $(\mathrm{Cs})$

cation $(\mathrm{C} 1 / \mathrm{C} 2 \mathrm{v})$

toluene (Cs)

$\mathrm{Cl}$

singlet carbene (Cs)

triplet carbene (Cs)

cation $(\mathrm{Cs} / \mathrm{C} 2 \mathrm{v})$

toluene $(\mathrm{C} 1 / \mathrm{Cs})$
Enthalpy

$-344.887961$

$-344.885256$

$-345.317642$

$-346.206695$

$-667.494670$

$-667.494602$

$-667.924423$

$-668.817201$

$-308.972498$

$-308.973748$

$-309.395811$

$-310.295312$

$-408.125396$

$-408.127774$

$-408.542081$

$-409.449969$

$-269.733859$

$-269.737248$

$-270.148084$

$-271.058922$

$-728.895960$

$-728.898186$

$-729.311071$

$-730.220808$

$-368.897063$

$-368.897421$

$-369.310824$

$-370.220126$
Free Energy

$-344.925854$

$-344.925206$

$-345.355767$

$-346.247340$

$-667.534761$

$-667.537070$

$-667.964242$

$-668.857514$

$-309.013834$

$-309.016508$

$-309.436744$

$-310.338750$

$-408.167374$

$-408.171544$

$-408.583810$

$-409.495981$

$-269.769278$

$-269.774168$

$-270.183853$

$-271.097378$

$-728.934772$

$-728.938625$

$-729.350189$

$-730.263099$

$-368.934525$

$-368.938625$

$-369.348669$

$-370.261662$ 
Table S2. Calculated G3MP2 enthalpies and free energies for singlet and triplet substituted arylcarbenes, benzylic cations, and toluenes, 298K. ${ }^{\mathrm{a}}$

Substituent (point group)

$\mathrm{CHO}$

singlet carbene, syn $(\mathrm{C} 1 / \mathrm{Cs})$

triplet carbene, syn $(\mathrm{C} 1 / \mathrm{Cs})$

cation $(\mathrm{C} 1 / \mathrm{Cs})$

toluene $(\mathrm{C} 1)$

$\mathrm{CF}_{3}$

singlet carbene $(\mathrm{C} 1 / \mathrm{Cs})$

triplet carbene $(\mathrm{C} 1)$

cation $(\mathrm{C} 1 / \mathrm{Cs})$

toluene $(\mathrm{ClCs})$

$\mathrm{CN}$

singlet carbene $(\mathrm{C} 1 / \mathrm{Cs})$

triplet carbene $(\mathrm{Cs})$

cation $(\mathrm{Cs} / \mathrm{C} 2 \mathrm{v})$

toluene $(\mathrm{C} 1 / \mathrm{Cs})$
Enthalpy

$-382.918336$

$-382.925543$

$-383.323626$

$-384.247410$

$-606.473877$

$-606.479914$

$-606.876315$

$-607.803920$

$-361.859551$

$-361.866549$

$-362.258230$

$-363.189357$
Free Energy

$-382.959036$

$-382.967482$

$-383.364817$

$-384.290470$

$-606.520520$

$-606.527560$

$-606.919153$

$-607.849109$

$-361.899589$

$-361.908096$

$-362.298644$

$-363.232975$

$\mathrm{NO}_{2}$

singlet carbene (Cs)

$-474.020145$

$-474.062878$

$-474.028074$

$-474.071911$

$-474.416035$

$-474.456412$

$-475.350963$

toluene $(\mathrm{C} 1 / \mathrm{Cs})$

$\underline{\text { miscellaneous arylcarbenes, arylcarbenium ions, and hydrogen adducts }}$

${ }^{1}$ methylphenylcarbene $\left(\mathrm{C}_{1}\right)$

${ }^{3}$ methylphenylcarbene $\left(\mathrm{C}_{\mathrm{s}}\right)$

1-phenylethyl ${ }^{+}\left(\mathrm{C}_{1} / \mathrm{C}_{\mathrm{s}}\right)$

ethylbenzene $\left(\mathrm{C}_{1}\right)$

${ }^{1}$ diphenylcarbene $\left(\mathrm{C}_{1} / \mathrm{C}_{2}\right)$

${ }^{3}$ diphenylcarbene $\left(\mathrm{C}_{1} / \mathrm{C}_{2}\right)$

diphenylmethyl ${ }^{+}\left(\mathrm{C}_{1} / \mathrm{C}_{2}\right)$

diphenylmethane $\left(\mathrm{C}_{1} / \mathrm{C}_{2}\right)$

${ }^{1}$ cycloheptatrienylCH ${ }^{+}(\mathrm{C} 1 / \mathrm{Cs})^{\mathrm{e}}$

${ }^{3}$ cycloheptatrienylCH$^{+}(\mathrm{C} 1 / \mathrm{Cs})^{\mathrm{e}}$

cycloheptatrienyl- $\mathrm{CH}_{2}{ }^{2+}(\mathrm{C} 1 / \mathrm{C} 2 \mathrm{v})$

$\left.\mathrm{Tr}^{+}-\mathrm{CH}_{3}(\mathrm{Cl} / \mathrm{Cs})\right)$
$-308.976428$

$-308.977731$

$-309.398541$

$-310.291540$

$-500.401930$

$-500.397719$

$-500.828936$

$-501.706233$

$-308.063758$

$-308.078349$

$-308.282367$

$-309.402742$
$-309.016947$

$-309.019702$

$-309.438369$

$-310.332061$

$-500.449636$

$-500.447984$

$-500.876368$

$-501.755817$

$-308.102283$

$-308.117701$

$-308.320943$

$-308.444426$ 
Table S2. Calculated G3MP2 enthalpies and free energies for singlet and triplet substituted arylcarbenes, arylcarbenium ions, and hydrogen adducts, 298K.

\section{Substituent (point group)}

Enthalpy

$-231.152477$

$-231.110355$

$-232.414305$

cyclopentadienyl- $\mathrm{CH}_{2}$ (fulvene), $\left(\mathrm{C}_{2 \mathrm{v}}\right)-231.771867$

${ }^{1}$ cyclopropenyl- $\mathrm{CH}^{+}(\mathrm{C} 2 \mathrm{v})^{\mathrm{g}}$

${ }^{3}$ cyclopropenyl- $\mathrm{CH}^{+}(\mathrm{Cs})^{\mathrm{g}}$

cyclopropenyl- $\mathrm{CH}_{2}{ }^{2+}$, bisected $\left(\mathrm{C}_{2 \mathrm{v}}\right)$

cyclopropenyl ${ }^{+}-\mathrm{CH}_{3}(\mathrm{C} 1 / \mathrm{Cs})$

${ }^{1}$ cyclopropenylidene $\left(\mathrm{C}_{2 \mathrm{v}}\right)^{\mathrm{h}}$

${ }^{3}$ cyclopropenylidene $\quad\left(\mathrm{C}_{2 \mathrm{v}}\right)^{\mathrm{h}}$

cyclopropenyl $^{+}\left(\mathrm{D}_{3 \mathrm{~h}}\right)$

cyclopropene $\left(\mathrm{C}_{2 \mathrm{v}}\right)$
$-153.474208$

$-153.441209$

$-153.559305$

$-154.773389$

$-115.155559$

$-115.041309$

$-115.514741$

$-116.400192$
Free Energy

$-231.186034$

$-231.145881$

$-232.450668$

$-231.805762$

$-153.504721$

$-153.472758$

$-153.589789$

$-154,806584$

$-115.182369$

$-115.069307$

$-115.541773$

$-116.428330$

an hartrees; 1 hartree $=627.51 \mathrm{kcal} / \mathrm{mol}$. See also Gronert, S.; Keeffe, J. R.; More O'Ferrall, R. A. J. Org. Chem. 2009, 74, 5250. ${ }^{\mathrm{b}}$ A slash indicates the structure has virtually the higher symmetry. In all the carbenes the carbenic $\mathrm{C}-\mathrm{H}$ bond is coplanar with the ring to within 0.5 degree, usually to within 0.1 degree. ${ }^{\mathrm{c}}$ The term syn indicates that the substituent group is coplanar with the ring, and oriented toward the $\mathrm{CH}$ hydrogen in the most stable structure as is the case for singlet and triplet $m-\mathrm{CH}=\mathrm{O}(\mathrm{C}=\mathrm{O}$ bond $)$, and the $\mathrm{C}-\mathrm{F}$ bond in $\mathrm{m}-\mathrm{CH}_{2} \mathrm{~F}$ singlet and cation, and for $p-\mathrm{O}-\mathrm{H}$ (triplet and singlet) and $\mathrm{S}-\mathrm{H}$ (singlet). The term anti indicates the substituent's orientation is coplanar with the ring and oriented preferentially away from the $\mathrm{CH}$ hydrogen as for singlet and triplet $m-\mathrm{OH}$, singlet $m-\mathrm{SH}$, and $m-\mathrm{CH}_{2} \mathrm{~F}$, cation, and for singlet and triplet $p-\mathrm{CH}=\mathrm{O}(\mathrm{C}=\mathrm{O})$ bond $)$. Gauche indicates neither syn nor anti as, for example, the triplet $m$-SH, the $m-\mathrm{C}-\mathrm{F}$ bond in $\mathrm{CH}_{2} \mathrm{~F}$, toluene, and for the $\mathrm{S}-\mathrm{H}$ bond in the $p$-triplet. ${ }^{\mathrm{d}} \mathrm{The} \mathrm{NH}_{2}$ group is pyramidal except in the cations where it is coplanar with the ring. ${ }^{\mathrm{e}}$ The carbenic $\mathrm{C}-\mathrm{H}$ unit is perpendicular to the ring in the singlet, and in the ring plane in the triplet. ${ }^{\mathrm{f}}$ The carbenic $\mathrm{C}-\mathrm{H}$ unit is bent and in the ring plane in the singlet, and perpendicular to the ring in the triplet. ${ }^{\mathrm{g}}$ The carbenic C-C-H unit is linear in the singlet, and is bent and in the ring plane in the triplet. ${ }^{\mathrm{h}}$ Bond lengths in the singlet are $1.329,1.427,1.427 \AA$, averaging $1.394 \pm 0.043$. In the triplet they are $1.337,1.457,1.457$, averaging $1.417 \pm 0.053 \AA$. In the triplet, there is a dramatic drop in the population of the beta spin electron on one of the carbons pi p-orbital. A node bisects the carbenic carbon and the shorter bond, with the unpaired electron of the pi-system localized on two of the carbons. 
Table S3. Calculated G3MP2 enthalpies and free energies for acyclic heteroatom- and cyano-substituted carbenes $\left(\mathrm{X}-\mathrm{CH}, \mathrm{X}_{2} \mathrm{C}\right)$, their conjugate acid carbocations $\left(\mathrm{X}-\mathrm{CH}_{2}{ }^{+}\right.$, $\underline{X}_{2} C^{+}$, and the hydrogenation products of the singlet carbenes, $298 \mathrm{~K} .^{a}$

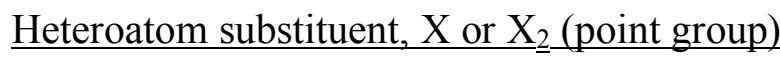

amino

carbene, singlet $\left(\mathrm{C}_{\mathrm{s}}\right)$

carbene, triplet $\left(\mathrm{C}_{1} / \mathrm{C}_{\mathrm{s}}\right)^{\mathrm{c}}$

cation $\left(\mathrm{C}_{2 \mathrm{v}}\right)$

aminomethane $\left(\mathrm{C}_{\mathrm{s}}\right)$

diamino

carbene, singlet $\left(\mathrm{C}_{2 \mathrm{v}}\right)$

carbene, triplet $\left(\mathrm{C}_{1}\right)$

cation $\left(\mathrm{C}_{2 \mathrm{v}}\right)$

diaminomethane $\left(\mathrm{C}_{1}\right)$

hydroxy

carbene, singlet, anti $\left(\mathrm{C}_{\mathrm{s}}\right)$

carbene, singlet, syn $\left(\mathrm{C}_{\mathrm{s}}\right)$

carbene, triplet $\left(\mathrm{C}_{1}\right)$

cation $\left(\mathrm{C}_{\mathrm{s}}\right)$

methanol $\left(\mathrm{C}_{\mathrm{s}}\right)$

methoxy

carbene, singlet $\left(\mathrm{C}_{1} / \mathrm{C}_{\mathrm{s}}\right)$

carbene, triplet $\left(\mathrm{C}_{1}\right)$

cation $\left(\mathrm{C}_{1} / \mathrm{C}_{\mathrm{s}}\right)$

methoxymethane $\left(\mathrm{C}_{1} / \mathrm{C}_{2 \mathrm{v}}\right)$

methoxy, methyl ${ }^{\mathrm{d}}$

carbene, singlet $\left(\mathrm{C}_{1} / \mathrm{C}_{\mathrm{s}}\right)$

carbene, triplet $\left(\mathrm{C}_{1}\right)$

cation $\left(\mathrm{C}_{1} / \mathrm{C}_{\mathrm{s}}\right)$

methoxyethane $\left(\mathrm{C}_{1}\right)$

dimethoxy

carbene, singlet $\left(\mathrm{C}_{1} / \mathrm{C}_{2 \mathrm{v}}\right)$

carbene, triplet $\left(\mathrm{C}_{1}\right)$

cation $\left(\mathrm{C}_{1} / \mathrm{C}_{\mathrm{s}}\right)$

dimethoxymethane $\left(\mathrm{C}_{1}\right)$

fluoro

carbene, singlet $\left(\mathrm{C}_{\mathrm{s}}\right)$

carbene, triplet $\left(\mathrm{C}_{\mathrm{s}}\right)$

cation $\left(\mathrm{C}_{2 \mathrm{v}}\right)$

fluoromethane $\left(\mathrm{C}_{3 \mathrm{v}}\right)$
Enthalpy

$-94.418137$

$-94.363160$

$-94.803643$

$-95.680373$

$-149.735223$

$-149.643828$

$-150.132983$

$-150.957875$

$-114.265735$

$-114.258381$

$-114.224891$

$-114.617745$

$-115.546803$

$-153.486245$

$-153.441506$

$-153.856324$

$-154.764705$

$-192.732530$

$-192.680467$

$-193.118961$

$-194.002110$

$-267.898677$

$-267.803324$

$-268.274132$

$-269.130363$

$-138.255744$

$-138.281045$

$-138.232451$

$-138.573018$

$-139.565138$
$-138.258692$

$-138.598051$

$-94.444005$

$-94.390627$

$-95.829102$

$-95.707627$

$-149.764784$

$-149.675665$

$-150.161555$

$-150.988365$

$-114.291250$

$-114.283931$

$-114.251949$

$-114.643599$

$-115.573787$

$-153.516175$

$-153.473048$

$-153.886692$

$-154.795430$

$-192.767564$

$-192.716627$

$-193.153791$

$-194.036497$

$-267.936473$

$-267.842607$

$-268.312094$

$-269.168036$

$-139.590375$ 
Table S3. Calculated G3MP2 enthalpies and free energies for acyclic heteroatom- and cyano-substituted carbenes $\left(\mathrm{X}-\mathrm{CH}, \mathrm{X}_{2} \mathrm{C}\right)$, their conjugate acid carbocations $\left(\mathrm{X}-\mathrm{CH}_{2}{ }^{+}\right.$, $\underline{X}_{2} \underline{C}^{+}$, and the hydrogenation products of the singlet carbenes, $298 \mathrm{~K}$. ${ }^{\text {a }}$

\begin{tabular}{|c|c|c|}
\hline 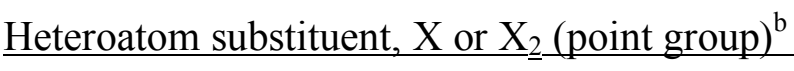 & Enthalpy & Free Energy \\
\hline \multicolumn{3}{|l|}{ difluoro } \\
\hline carbene, singlet $\left(\mathrm{C}_{2 \mathrm{v}}\right)$ & -237.472104 & -237.499393 \\
\hline carbene, triplet $\left(\mathrm{C}_{2 \mathrm{v}}\right)$ & -237.382459 & -237.410931 \\
\hline cation $\left(\mathrm{C}_{2 \mathrm{v}}\right)$ & -237.752071 & -2237.779496 \\
\hline difluoromethane $\left(\mathrm{C}_{2 \mathrm{v}}\right)$ & -238.733471 & -238.761428 \\
\hline \multicolumn{3}{|l|}{ phosphino } \\
\hline carbene, singlet $\left(\mathrm{C}_{1} / \mathrm{C}_{\mathrm{s}}\right)$ & -380.621801 & -380.650027 \\
\hline carbene, triplet $\left(\mathrm{C}_{1} / \mathrm{C}_{\mathrm{s}}\right)^{\mathrm{c}}$ & -380.600403 & -380.630063 \\
\hline cation $\left(\mathrm{C}_{1} / \mathrm{C}_{2 \mathrm{v}}\right)$ & -380.993967 & -381.022489 \\
\hline phosphinomethane $\left(\mathrm{C}_{\mathrm{s}}\right)$ & -381.922034 & -381.951323 \\
\hline \multicolumn{3}{|l|}{ sulfhydryl (thio) } \\
\hline carbene, singlet, anti $\left(\mathrm{C}_{\mathrm{s}}\right)$ & -436.878179 & -436.905308 \\
\hline carbene, singlet, syn $\left(\mathrm{C}_{\mathrm{s}}\right)$ & -436.876251 & -436.903405 \\
\hline carbene, triplet $\left(\mathrm{C}_{1}\right)$ & -436.845312 & -436.874047 \\
\hline cation $\left(\mathrm{C}_{\mathrm{s}}\right)$ & -437.239391 & -437.266785 \\
\hline methanethiol $\left(\mathrm{C}_{\mathrm{s}}\right)$ & -438.164939 & -438.193701 \\
\hline \multicolumn{3}{|l|}{ methylthio } \\
\hline carbene, singlet $\left(\mathrm{C}_{1} / \mathrm{C}_{\mathrm{s}}\right)$ & -476.117188 & -476.149135 \\
\hline carbene, triplet $\left(\mathrm{C}_{1}\right)$ & -476.077456 & -476.110665 \\
\hline cation $\left(\mathrm{C}_{1} / \mathrm{C}_{\mathrm{s}}\right)$ & -476.491272 & -476.524014 \\
\hline methylthiomethane $\left(\mathrm{C}_{1} / \mathrm{C}_{2 \mathrm{v}}\right)$ & -477.394868 & -477.427742 \\
\hline \multicolumn{3}{|l|}{ chloro } \\
\hline carbene, singlet $\left(\mathrm{C}_{\mathrm{s}}\right)$ & -498.252971 & -498.279628 \\
\hline carbene, triplet $\left(\mathrm{C}_{\mathrm{s}}\right)$ & -498.241909 & -498.269415 \\
\hline cation $\left(\mathrm{C}_{2 \mathrm{v}}\right)$ & -498.592261 & -498.618544 \\
\hline chloromethane $\left(\mathrm{C}_{3 \mathrm{v}}\right)$ & -499.568852 & -499.595425 \\
\hline \multicolumn{3}{|l|}{ dichloro } \\
\hline carbene, singlet $\left(\mathrm{C}_{2 \mathrm{v}}\right)$ & -957.437542 & -957.467576 \\
\hline carbene, triplet $\left(\mathrm{C}_{2 \mathrm{v}}\right)$ & -957.402860 & -957.433884 \\
\hline cation $\left(\mathrm{C}_{2 \mathrm{v}}\right)$ & -957.767645 & -957.797600 \\
\hline dichloromethane $\left(\mathrm{C}_{2 \mathrm{v}}\right)$ & -958.722710 & -958.753338 \\
\hline \multicolumn{3}{|l|}{ silylmethyl $\left(\mathrm{H}_{3} \mathrm{SiCH}_{2}\right)$} \\
\hline carbene, singlet ${ }^{\mathrm{e}}$ & NA & NA \\
\hline carbene, triplet $\left(\mathrm{C}_{1}\right)$ & -368.571863 & -368.606655 \\
\hline silylethene $\left(\mathrm{C}_{1} / \mathrm{C}_{\mathrm{s}}\right)^{\mathrm{e}}$ & -368.689821 & -368.723016 \\
\hline
\end{tabular}


cation $\left(\mathrm{C}_{1} / \mathrm{C}_{\mathrm{s}}\right)^{\mathrm{e}}$

silylethane $\left(\mathrm{C}_{1} / \mathrm{C}_{\mathrm{s}}\right)$
$-368.983056$

$-369.899776$
$-369.015720$

$-369.933734$

Table S3. Calculated G3MP2 enthalpies and free energies for acyclic heteroatom- and cyano-substituted carbenes $\left(\mathrm{X}-\mathrm{CH}, \mathrm{X}_{2} \mathrm{C}\right)$, their conjugate acid carbocations $\left(\mathrm{X}-\mathrm{CH}_{2}{ }^{+}\right.$, $\underline{\mathrm{X}}_{2} \underline{C}^{+}$, and the hydrogenation products of the singlet carbenes, $298 \mathrm{~K}$.

Heteroatom substituent, $\mathrm{X}$ or $\mathrm{X}_{2}$ (point group) $^{\mathrm{b}} \quad$ Enthalpy

silyl $\left(\mathrm{H}_{3} \mathrm{Si}\right)$

carbene, singlet $\left(\mathrm{C}_{1}\right)$

carbene, singlet ts $\left(\mathrm{C}_{1}\right)^{\mathrm{f}}$

carbene, triplet $\left(\mathrm{C}_{1} / \mathrm{C}_{\mathrm{s}}\right)$

cation $\left(\mathrm{C}_{1} / \mathrm{C}_{\mathrm{s}}\right)^{\mathrm{g}}$

silylmethane $\left(\mathrm{C}_{3 \mathrm{v}}\right)$

disilyl $\left(\mathrm{H}_{3} \mathrm{Si}\right)_{2}$

carbene, singlet $\left(\mathrm{C}_{1} / \mathrm{C}_{\mathrm{s}}\right)$

carbene, triplet $\left(\mathrm{C}_{1} / \mathrm{D}_{3} \mathrm{~h}\right)$

cation $^{\mathrm{h}}\left(\mathrm{C}_{2 \mathrm{v}}\right)$

disilylmethane $\left(\mathrm{C}_{1} / \mathrm{C}_{2 \mathrm{v}}\right)$

nitroso $(\mathrm{O}=\mathrm{N})^{\mathrm{i}}$

carbene, singlet $\left(\mathrm{C}_{\infty \mathrm{v}}\right)$

carbene, triplet, syn $\left(\mathrm{C}_{\mathrm{s}}\right)$

cation $\left(\mathrm{C}_{2 \mathrm{v}}\right)$

nitrosomethane $\left(\mathrm{C}_{1} / \mathrm{C}_{\mathrm{s}}\right)$

diaminomethyl $^{+}$

carbene, singlet $\left(\mathrm{C}_{1}\right)$

carbene, singlet, ts $\left(\mathrm{C}_{2}\right)^{\mathrm{j}}$

carbene, triplet $\left(\mathrm{C}_{1} / \mathrm{C}_{\mathrm{s}}\right)$

dication $\left(\mathrm{C}_{1} / \mathrm{C}_{2 \mathrm{v}}\right)$

1,1-diaminoethyl ${ }^{+}\left(\mathrm{C}_{1} / \mathrm{C}_{\mathrm{s}}\right)$

imino $(\mathrm{HN}=)^{\mathrm{k}}$

carbene, singlet $\left(\mathrm{C}_{\infty \mathrm{v}}\right)$

carbene, triplet $\left(\mathrm{C}_{\mathrm{s}}\right)$

cation $\left(\mathrm{C}_{\infty \mathrm{v}}\right)$

formaldimine $\left(\mathrm{C}_{\mathrm{s}}\right)$

oxo $(\mathrm{O}=)^{1}$

carbene, singlet $\left(\mathrm{C}_{\infty \mathrm{v}}\right)$

carbene, triplet $\left(\mathrm{C}_{\infty \mathrm{v}}\right)$

cation $\left(\mathrm{C}_{\infty \mathrm{v}}\right)$

formaldehyde $\left(\mathrm{C}_{2 \mathrm{v}}\right)$

cyano

carbene, singlet $\left(\mathrm{C}_{\mathrm{s}}\right)$
$-329.318535$

$-329.319669$

$-329.347351$

$-329.756741$

$-330.672257$

$-619.583421$

$-619.618423$

$-620.039501$

$-620.931153$

$-168.361078$

$-168.264792$

$-168.623743$

$-169.562238$

$-188.025054$

$-188.013890$

$-188.044379$

$-188.169612$

$-189.383023$

$-93.272356$

$-93.102080$

$-93.564035$

$-94.475420$

$-113.185455$

$-112.965319$

$-113.409011$

$-114.348622$

$-131.204112$
Free Energy

$-329.348210$

$-329.348245$

$-329.378466$

$-329.787215$

$-330.702415$

$-619.620268$

$-619.658157$

$-620.073111$

$-620.968131$

$-168.386568$

$-168.293841$

$-168.651761$

$-169.591776$

$-188.058079$

$-188.046179$

$-188.077590$

$-188.204254$

$-189.416879$

$-93.295578$

$-93.127896$

$-93.587267$

$-94.501158$

$-113.207857$

$-112.988898$

$-113.431831$

$-114.373393$

$-131.231848$ 
carbene, triplet $\left(\mathrm{C}_{\mathrm{s}}\right)$

$-131.219901$

$-131.248381$

cation $\left(\mathrm{C}_{2 \mathrm{v}}\right)$

$-131.510055$

$-131.537901$

acetonitrile $\left(\mathrm{C}_{\mathrm{s}} / \mathrm{C}_{3 \mathrm{v}}\right)$

$-132.540533$

$-132.569076$

Table S3. Calculated G3MP2 enthalpies and free energies for acyclic heteroatom- and cyano-substituted carbenes $\left(\mathrm{X}-\mathrm{CH}, \mathrm{X}_{2} \mathrm{C}\right)$, their conjugate acid carbocations $\left(\mathrm{X}-\mathrm{CH}_{2}{ }^{+}\right.$, $\underline{X}_{2} \underline{C}^{+}$, and the hydrogenation products of the singlet carbenes, $298 \mathrm{~K} .{ }^{a}$

Heteroatom substituent, $\mathrm{X}$ or $\mathrm{X}_{2}$ (point group)

Enthalpy

Free Energy

dicyano

carbene, singlet $\left(\mathrm{C}_{2 \mathrm{v}}\right)$

$-223.334049$

$-223.366463$

carbene, triplet $\left(\mathrm{C}_{2 \mathrm{v}} / \mathrm{D}_{\infty \mathrm{h}}\right)$

$-223.352783$

$-223.383524$

cation $\left(\mathrm{C}_{\mathrm{s}} / \mathrm{C}_{2 \mathrm{v}}\right)$

$-223.618017$

$-223.651786$

dicyanomethane $\left(\mathrm{C}_{2 \mathrm{v}}\right)$

$-224.651325$

$-224.684100$

nitro

carbene, singlet ${ }^{\mathrm{m}}$

carbene, triplet $\left(\mathrm{C}_{1} \mathrm{C}_{\mathrm{s}}\right)$

cation $\left(\mathrm{C}_{1} \mathrm{C}_{\mathrm{s}}\right)^{\mathrm{n}}$

$-243.360457$

$-243.391741$

$-243.700451$

$-243.730423$

nitromethane $\left(\mathrm{C}_{1} \mathrm{C}_{\mathrm{s}}\right)$

$-244.699114$

$-244.732671$

dinitro

carbene, singlet $\left(\mathrm{C}_{1}\right)^{\mathrm{n}}$

$-447.655041$

$-447.694872$

carbene, triplet $\left(\mathrm{C}_{1} / \mathrm{C}_{2}\right)$

$-447.623663$

$-447.662894$

cation $\left(\mathrm{C}_{1}\right)^{\mathrm{n}}$

$-447.946742$

$-447.984000$

dintromethane $\left(\mathrm{C}_{1} / \mathrm{C}_{\mathrm{s}}\right)$

$-448.970014$

$-449.009531$

amino, cyano

carbene, singlet $\left(\mathrm{C}_{1} / \mathrm{C}_{\mathrm{s}}\right)$

$-186.553559$

$-186.584431$

carbene, triplet $\left(\mathrm{C}_{1} / \mathrm{C}_{\mathrm{s}}\right)$

$-186.501973$

$-186.535445$

cation $\left(\mathrm{C}_{\mathrm{s}}\right)$

$-186.900293$

$-186.931462$

aminocyanomethane $\left(\mathrm{C}_{1}\right)$

$-187.804322$

$-187.836384$

amino, nitro

carbene, singlet $\left(\mathrm{C}_{1}\right)$

$-298.719662$

$-298.753902$

carbene, triplet $\left(\mathrm{C}_{1}\right)$

$-298.653582$

$-298.689412$

cation $\left(\mathrm{C}_{1} / \mathrm{C}_{\mathrm{s}}\right)$

$-299.056169$

$-299.089655$

aminonitromethane $\left(\mathrm{C}_{1} / \mathrm{C}_{\mathrm{s}}\right)$

$-299.978719$

$-300.013659$

${ }^{\mathrm{a}}$ In hartrees; 1 hartree $=627.51 \mathrm{kcal} / \mathrm{mol} .{ }^{\mathrm{b}} \mathrm{A}$ slash indicates the structure has virtually the higher symmetry. ${ }^{\mathrm{c}}$ These triplets have pyramidal $\mathrm{NH}_{2}\left(\mathrm{PH}_{2}\right)$ group. The $\mathrm{C}-\mathrm{H}$ bond is perpendicular and anti to the $\mathrm{NH}_{2}\left(\mathrm{PH}_{2}\right)$ plane. ${ }^{\mathrm{d}}$ These species have two substituents, methoxy and methyl. ${ }^{\mathrm{e}}$ singlet silymethylcarbene rearranges to silylethene upon optimization at the MP2 level. The silylethyl cation is a symmetrical, silyl-bridged structure. ${ }^{\mathrm{f}}$ This is the transition state for rotation about the Si-C bond. $\Delta H^{\ddagger}=-0.7 \mathrm{kcal} / \mathrm{mol}$ and $\Delta G^{\ddagger}=0.0 \mathrm{kcal} / \mathrm{mol}$. ${ }^{\mathrm{g}} \mathrm{The}$ silylmethyl cation, $\mathrm{H}_{3} \mathrm{SiCH}_{2}{ }^{+}$, rearranges to the methylsilyl cation, $\mathrm{CH}_{3} \mathrm{SiH}_{2}{ }^{+}$, upon optimization. ${ }^{\mathrm{h}}$ The cation rearranges to a symmetrical $\left(\mathrm{C}_{2 \mathrm{v}}\right)$ bridged structure, a hydrogen bridging the two silicons. A second cation, $\mathrm{H}_{3} \mathrm{Si}-\mathrm{CH}_{2}-\mathrm{SiH}_{2}{ }^{+}$, is $10.6 \mathrm{kcal} / \mathrm{mol}$ less stable. ${ }^{\mathrm{i}}$ The singlet carbene is hydrogen cyanide- $N$-oxide, 
$\mathrm{O}-\mathrm{N} \equiv \mathrm{C}-\mathrm{H}$. The cation is $\mathrm{O}=\mathrm{N}=\mathrm{CH}_{2}{ }^{+}$. ${ }^{\mathrm{j}}$ This ts has a linear $\mathrm{CCH}$ structure, and corresponds to 2,2-diaminovinyl cation, iv $=623 \mathrm{~cm}^{-1}$ (CCH bending). ${ }^{\mathrm{k}} \mathrm{The}$ singlet carbene is hydrogen isocyanide, $\mathrm{HN} \equiv \mathrm{C}$. The cation is protonated hydrogen cyanide, $\mathrm{HN} \equiv \mathrm{CH}^{+}$. ${ }^{1}$ The singlet carbene is carbon monoxide. See also Gronert, S.; Keeffe, J. R.; More O'Ferrall, R. A. J. Org. Chem. 2009, 74, 5250. ${ }^{\mathrm{m}}$ This structure is that of nitrosocarbaldehyde $\left(\mathrm{C}_{1}\right)$. The $\mathrm{C}-\mathrm{N}-\mathrm{O}$ angle is $110.0^{\circ}$, and the $\mathrm{O}-\mathrm{C}-\mathrm{N}-\mathrm{O}$ dihedral is $25.7^{\circ}$. ${ }^{\mathrm{n}} \mathrm{An}$ oxygen from a nitro group partially bridges to the carbon in the cations, and in the dinitro singlet. 
Table S4. Calculated G3MP2 enthalpies and free energies for miscellaneous 1,3diheterocyclic carbenes, their $\mathrm{C} 2$ conjugate acids (carbene- $\mathrm{CH}^{\overline{ }}{ }^{\overline{ }}$ ), the $\mathrm{C} 2$ hydrogenation products of the carbenes (carbene- $\mathrm{CH}_{2}$ ), and their 4,5-dihydro analogs (carbeneH 2 298K.

Carbene, (point group) $)^{b}$ Enthalpy Free Energy

Set $1, X=Y=N H$

1. imidazol-2-ylidene, singlet $\left(\mathrm{C}_{2 \mathrm{v}}\right)$

$-225.820192$

$-225.850622$

1. triplet, cis $\left(\mathrm{C}_{1} \mathrm{C}_{\mathrm{s}}\right)^{\mathrm{c}}$

$-225.683581$

$-225.716425$

triplet ts $\left(\mathrm{C}_{2 \mathrm{v}}\right)^{\mathrm{c}}$

$-225.653707$

$-225.687258$

imidazole $\left(\mathrm{C}_{\mathrm{s}}\right)$

$-225.861555$

$-225.892512$

1-CH $\mathbf{C H}^{+}$(imidazolium +$)\left(\mathrm{C}_{2 \mathrm{v}}\right)^{\mathrm{c}, \mathrm{d}}$

$-226.218697$

$-226.249074$

1-CH $\mathbf{C H}_{2}$ cis-1,3-diazacyclopent-4-ene $)\left(\mathrm{C}_{1} / \mathrm{C}_{\mathrm{s}}\right)^{\mathrm{d}} \quad-227.010068$

$-227.042386$

1-C $\mathrm{CH}_{2}$ trans-1,3-diaza-cyclopent-4-ene) $\left(\mathrm{C}_{1}\right)^{\mathrm{d}} \quad-227.010676$

$-227.042947$

1 $\mathbf{H}_{2}$. 4,5-dihydroimidazol-2-ylidene, singlet $\left(\mathrm{C}_{1} / \mathrm{C}_{2}\right)^{\mathrm{e}}-227.000781$

$-227.033920$

$1 \mathrm{H} 2$ triplet $\left(\mathrm{C}_{1}\right)$

$-226.885642$

triplet ts $\left(\mathrm{C}_{1} / \mathrm{C}_{2}\right)^{\mathrm{f}}$

$-227.001779$

$-226.919711$

$-227.033594$

$\mathbf{1} \mathbf{H}_{2}-\mathbf{C H}^{+}\left(\mathrm{C}_{2}\right)$

$-227.403599$

$-227.436440$

$\mathbf{1 H}_{2}-\mathbf{N H}^{+}\left(\mathrm{C}_{1}\right)$

$-227.325872$

$-227.358944$

$\mathbf{1 H}_{\mathbf{2}}-\mathbf{C H}_{2}$, (cis-1,3-diazacyclopentane) $\left(\mathrm{C}_{\mathrm{s}}\right)^{\mathrm{g}} \quad-228.216362$

$-228.250115$

$\mathbf{1 H}_{2}-\mathbf{C H}_{2}$, (trans-1,3-diazacyclopentane) $\left(\mathrm{C}_{1} / \mathrm{C}_{2}\right)^{\mathrm{g}}-228.215533$

$-228.249829$

Set 2, $X=Y=O$

2. 1,3-dioxol-2-ylidene, singlet $\left(\mathrm{C}_{2 \mathrm{v}}\right)$

2. triplet $\left(\mathrm{C}_{1}\right)$

2- $\mathbf{C H}^{+}\left(\mathrm{C}_{2 \mathrm{v}}\right)$

$\mathbf{2}-\mathbf{O H}^{+}\left(\mathrm{C}_{1} / \mathrm{C}_{\mathrm{s}}\right)^{\mathrm{h}}$

2- $\mathbf{C H}_{2}$ (1,3-dioxole) $\left(\mathrm{C}_{1} / \mathrm{C}_{\mathrm{s}}\right)^{\mathrm{I}}$

2- $\mathbf{C H}_{2}$, ts $\left(\mathrm{C}_{2 \mathrm{v}}\right)^{\mathrm{I}}$
$-265.519783$

$-265.381822$

$-265.871359$

$-265.778152$

$-266.740024$

$-266.739573$

2 $\mathbf{H}_{2}$. 4,5-dihydro-1,3-dioxol-2-ylidene $\left(\mathrm{C}_{2} / \mathrm{C}_{2 \mathrm{v}}\right)$

$2 \mathbf{H}_{2}-\mathbf{C H}^{+}\left(\mathrm{C}_{1} / \mathrm{C}_{2 \mathrm{v}}\right)$

$\mathbf{2} \mathbf{H}_{2}-\mathbf{C H}_{2}$ (1,3-dioxolane) $\left(\mathrm{C}_{2}\right)^{\mathrm{j}}$

$2 \mathrm{H}_{2}-\mathbf{O H}^{+}$
$-266.720970$

$-267.085185$

$-267.948326$

$\mathrm{NA}^{\mathrm{k}}$
$-265.549762$

$-265.415100$

$-265.901403$

$-265.811179$

$-266.772172$

$-267.769771$

$-266.753058$

$-267.117807$

$-267.982335$

$\mathrm{NA}^{\mathrm{k}}$

Set 3, $X=O, Y=N H$

3. 1-oxa-3-azacyclopent-4-ene-2-ylidene,

$-245.668231$

$-245.699084$ singlet $\left(\mathrm{C}_{1} / \mathrm{C}_{\mathrm{s}}\right)$

3. triplet $\left(\mathrm{C}_{1}\right)$

$-245.529957$

$-245.563568$

3- $\mathbf{C H}^{+}\left(\mathrm{C}_{1} / \mathrm{C}_{\mathrm{s}}\right)$

$-246.046399$

$-246.077237$

3-OH ${ }^{+}\left(\mathrm{C}_{1} / \mathrm{C}_{\mathrm{s}}\right)^{1}$

$-245.934554$

$-245.966623$

3- $\mathbf{C H}_{2}$ (1-oxa-3-aza-cyclopent-4-ene) $\left(\mathrm{C}_{1}\right)^{\mathrm{m}}$

$-246.876764$

$-246.908697$ 
Table S4. Calculated G3MP2 enthalpies and free energies for miscellaneous 1,3diheterocyclic carbenes, their $\mathrm{C} 2$ conjugate acids (carbene- $\mathrm{CH}^{\overline{ }}{ }^{\overline{ }}$ ), the $\mathrm{C} 2$ hydrogenation products of the carbenes (carbene- $\mathrm{CH}_{2} 2$, and their 4,5-dihydro analogs (carbene ${ }_{2} 2 \underline{298 K}{ }^{a}$

Carbene, (point group) $)^{\mathrm{b}}$ Enthalpy Free Energy

3H 2 . 1-oxa-3-azacyclopentane-2-ylidene $\left(\mathrm{C}_{1}\right)^{\mathrm{n}} \quad-246.860054$ $-246.893580$ $3 \mathbf{H}_{2}-\mathbf{C H}^{+}\left(\mathrm{C}_{1} / \mathrm{C}_{\mathrm{s}}\right)$ $-247.246818$ $-247.279694$ $\mathbf{3 H}_{2}-\mathbf{O H}^{+}\left(\mathrm{C}_{1}\right)^{\mathrm{O}}$ $-247.152554$ $-247.185718$ $\mathbf{3 H}_{2}-\mathbf{C H}_{2}$ (1-oxa-3-azacyclopentane) $\left(\mathrm{C}_{1}\right)^{\mathrm{p}}$ $-248.084336$ $-248.117893$

Set $\mathbf{4}, \mathrm{X}=\mathrm{Y}=\mathrm{PH}$

4. 1,3-diphosphol-2-ylidene, singlet, cis

$-798.232022$

$-798.267028$ $\left(\mathrm{C}_{1} / \mathrm{C}_{\mathrm{s}}\right)^{\mathrm{q}}$

4, triplet, cis $\left(\mathrm{C}_{1} / \mathrm{C}_{\mathrm{s}}\right)^{\mathrm{q}}$

$-798.195012$

$-798.231277$

4, singlet, trans $\left(\mathrm{C}_{1} / \mathrm{C}_{2}\right)^{\mathrm{q}}$

$-798.237437$

$-798.272789$

4, triplet, trans $\left(\mathrm{C}_{1} / \mathrm{C}_{2}\right)^{\mathrm{q}}$

$-798.195832$

$-798.232195$

4-CH $\mathbf{H}^{+}$, trans $\left(\mathrm{C}_{1} / \mathrm{C}_{2}\right)^{\mathrm{q}}$

$-798.624664$

$-798.659885$

4-CH', cis, ts $\left(\mathrm{C}_{\mathrm{s}}\right)^{\mathrm{q}}$

$-798.618174$

$-798.652924$

4- $\mathrm{CH}_{2}$, cis $\left(\mathrm{C}_{\mathrm{s}}\right)^{\mathrm{q}}$

$-799.515194$

$-799.551134$

4-CH $\mathrm{CH}_{2}$, trans $\left(\mathrm{C}_{1}\right)^{\mathrm{q}}$

$-799.515650$

$-799.549970$

4H $\mathbf{H}_{2}$ 4,5-dihydro-1,3-diphospholane-

$-799.429888$

$-799.466193$

2-ylidene, singlet, cis $\left(\mathrm{C}_{1}\right)^{\mathrm{q}, \mathrm{r}}$

$\mathbf{4} \mathbf{H}_{2}$, triplet, cis $\left(\mathrm{C}_{1}\right)^{\mathrm{q}, \mathrm{r}}$

$-799.403330$

$-799.440624$

$\mathbf{4} \mathbf{H}_{2}$, singlet, trans $\left(\mathrm{C}_{1} / \mathrm{C}_{2}\right)^{\mathrm{q}}$

$\mathbf{4} \mathbf{H}_{2}$, triplet, trans $\left(\mathrm{C}_{1} / \mathrm{C}_{2}\right)^{\mathrm{q}}$

$-799.427012$

$-799.472891$

$-799.401923$

$-799.439494$

$\mathbf{4} \mathbf{H}_{2}-\mathbf{C H}^{+}$, cis $\left(\mathrm{C}_{1}\right)^{\mathrm{q}, \mathrm{r}}$

$-799.821593$

$-799.858274$

$\mathbf{4} \mathbf{H}_{2}-\mathbf{C H}^{+}$, trans $\left(\mathrm{C}_{1} / \mathrm{C}_{2}\right)^{\mathrm{q}}$

$-799.827474$

$-799.864200$

$\mathbf{4} \mathbf{H}_{2}-\mathbf{C H}_{2}$, cis $\left(\mathrm{C}_{1}\right)^{\mathrm{q}, \mathrm{r}}$

$-800.723018$

$-800.760129$

$\mathbf{4} \mathbf{H}_{2}-\mathbf{C H}_{2}$, trans $\left(\mathrm{C}_{1}\right)^{\mathrm{q}, \mathrm{r}}$

$-800.719621$

$-800.756648$

Set 5, $X=Y=S$

5. 1,3-dithiol-2-ylidene, singlet $\left(\mathrm{C}_{2 \mathrm{v}}\right)$

5, triplet $\left(\mathrm{C}_{\mathrm{s}}\right)$

5- $\mathbf{C H}^{+}\left(\mathrm{C}_{2 \mathrm{v}}\right)$

5- $\mathbf{C H}_{2}\left(\mathrm{C}_{\mathrm{s}}\right)$

$\mathbf{5} \mathbf{H}_{2}$ 1,3-dithiolan-2-ylidene, singlet $\left(\mathrm{C}_{2}\right)$

$\mathbf{4} \mathbf{H}_{2}$ triplet $\left(\mathrm{C}_{1}\right)$

$4 \mathbf{H}_{2}-\mathbf{C H}^{+}\left(\mathrm{C}_{2}\right)$

$\mathbf{4} \mathrm{H}_{2}-\mathbf{C H}_{2}\left(\mathrm{C}_{2}\right)$
$-910.768219$

$-910.677468$

$-911.141533$

$-911.991562$

$-911.955024$

$-911.879860$

$-912.329966$

$-913.196354$
$-910.801497$

$-910.714564$

$-911.174828$

$-912.026526$

$-911.989273$

$-911.917052$

$-912.364793$

$-913.233456$ 
Table S4. Calculated G3MP2 enthalpies and free energies for miscellaneous 1,3diheterocyclic carbenes, their $\mathrm{C} 2$ conjugate acids (carbene- $\mathrm{CH}^{\overline{ }}{ }^{\overline{ }}$ ), the $\mathrm{C} 2$ hydrogenation products of the carbenes (carbene- $\mathrm{CH}_{2}$ ), and their 4,5-dihydro analogs (carbeneH $\underline{2}_{2} \underline{298 K}^{\mathrm{a}}$

Carbene, (point group) $)^{b}$ Enthalpy Free Energy

Set 6, $\mathrm{X}=\mathrm{Y}=\mathrm{NCH}_{3}$

6. $N, N$ '-dimethyl-2-imidazol-2-ylidene

$-304.276053$

$-304.315097$ singlet $\left(\mathrm{C}_{1} / \mathrm{C}_{2 \mathrm{v}}\right)$

6. triplet $\left(\mathrm{C}_{1} \mathrm{C}_{\mathrm{s}}\right)$

$-304.136531$

$-304.176819$

6-CH $\mathbf{C H}^{+}\left(\mathrm{C}_{1} / \mathrm{C}_{2 \mathrm{v}}\right)$

$-304.687816$

$=304.727437$

6- $\mathrm{CH}_{2} \operatorname{cis}\left(\mathrm{C}_{1} / \mathrm{C}_{\mathrm{s}}\right)$

$-305.466380$

$-305.505389$

Set 7, 4,5-dimethyl, $\mathrm{X}=\mathrm{Y}=\mathrm{NH}$

7. 4,5-dimethylimidazol-2-ylidene, singlet $\left(\mathrm{C}_{1} / \mathrm{C}_{2 \mathrm{v}}\right) \quad-304.297092$

$-304.336101$

7. triplet $\left(\mathrm{C}_{1} \mathrm{C}_{\mathrm{s}}\right)$

$-304.163859$

$-304.204163$

7-CH' $\left(\mathrm{C}_{1} / \mathrm{C}_{2 \mathrm{v}}\right)$

$-304.707585$

$-304.746958$

7- $\mathbf{C H}_{2}\left(\mathrm{C}_{1} / \mathrm{C}_{\mathrm{s}}\right)$

$-305.490261$

$-305.529965$

Set 8, 4,5-difluoro, $\mathrm{X}=\mathrm{Y}=\mathrm{NH}$

8. 4,5-difluoroimidazol-2-ylidene, singlet $\left(\mathrm{C}_{2 \mathrm{v}}\right) \quad-424.115589$

$-424.150520$

8. triplet, trans $\mathrm{C}-\mathrm{F}$ bonds $\left(\mathrm{C}_{1} / \mathrm{C}_{2}\right)$

$-424.010714$

$-424.048324$

8. triplet, ts $\left(\mathrm{C}_{2 \mathrm{v}}\right)^{\mathrm{s}}$

$-423.965863$

$-424.003280$

8- $\mathbf{C H}^{+}\left(\mathrm{C}_{2 \mathrm{v}}\right)$

$-424.496013$

$-424.530746$

8- $\mathrm{CH}_{2} \operatorname{cis}\left(\mathrm{C}_{1} / \mathrm{C}_{\mathrm{s}}\right)^{\mathrm{t}}$

$-425.321175$

$-425.357596$

Set 9, 4,5-dimethyl, $\mathrm{X}=\mathrm{Y}=\mathrm{NCH}_{3}$

9. 4,5,N,N'-tetramethylimidazol-2-

$-382.753558$

$-382.799488$

ylidene, singlet $\left(\mathrm{C}_{1} / / \mathrm{C}_{2 \mathrm{v}}\right)$

9. triplet, trans, twisted ring $\left(\mathrm{C}_{1} / \mathrm{C}_{\mathrm{s}}\right)^{\mathrm{t}}$

$-382.616075$

$-382.663618$

8-CH $\mathbf{H}^{+}\left(\mathrm{C}_{1} / / \mathrm{C}_{2 \mathrm{v}}\right)$

$-383.175874$

$-383.222420$

$\mathbf{8}-\mathrm{CH}_{2}$, trans, twisted ring $(\mathrm{C} 1)^{\mathrm{t}}$

$-383.943265$

$-383.989279$

Set 10, 4,5-disilyl, $X=Y=\mathrm{SiH}_{3}$

10. $4,5, N, N$ '-tetrasilylimmidazol-2-

$-1386.894560$

$-1386.950521$

ylidene, singlet $\left(\mathrm{C}_{1} / / \mathrm{C}_{2 \mathrm{v}}\right)$

10. triplet, trans, twisted ring $\left(\mathrm{C}_{1}\right)^{\mathrm{t}}$

$-1386.762263$

$-1386.822519$

10- $\mathbf{C H}^{+}\left(\mathrm{C}_{1}\right)$

$-1387.306083$

$-1387.361728$

10- $\mathrm{CH}_{2}$, cis $\left(\mathrm{C}_{1}\right)^{\mathrm{t}}$

$-1388.088095$

$-1389.147253$ 
Table S4. Calculated G3MP2 enthalpies and free energies for miscellaneous 1,3diheterocyclic carbenes, their $\mathrm{C} 2$ conjugate acids (carbene- $\mathrm{CH}^{\overline{ }}{ }^{\overline{ }}$ ), the $\mathrm{C} 2$ hydrogenation products of the carbenes (carbene- $\mathrm{CH}_{2}$ ), and their 4,5-dihydro analogs (carbeneH 2 298K.

Carbene, (point group) $)^{b}$ Enthalpy Free Energy

Set 11, 3-membered ring, $\mathrm{X}=\mathrm{Y}=\mathrm{NH}$

$\begin{array}{lll}\text { 11. 1,2-diaziridin-3-ylidene, singlet, trans }\left(\mathrm{C}_{2}\right)^{\mathrm{t}} & -148.432232 & -148.459403 \\ \text { 11. singlet, ts }\left(\mathrm{C}_{2 \mathrm{v}}\right)^{\mathrm{u}} & -148.372102 & -148.399243 \\ \text { 11. triplet, trans }\left(\mathrm{C}_{2}\right)^{\mathrm{t}} & -148.344558 & -148.372626 \\ \text { 11-CH } & -148.755714 & -148.784703 \\ \left.\text { 11-CHa } \mathbf{C H}^{+} \text {, cis }\left(\mathrm{C}_{\mathrm{s}}\right)^{\mathrm{t}} / \mathrm{C} 2\right)^{\mathrm{t}} & -148.747795 & -148.776897 \\ \text { 11-CH } & -148.713598 & -148.741614 \\ \text { 11-C } \mathbf{C H}_{2} \text {, trans }\left(\mathrm{C}_{2}\right)^{\mathrm{t}} & -149.701610 & -149.729031\end{array}$

Set 12, 4-membered ring, $\mathrm{X}=\mathrm{Y}=\mathrm{NH}$

12. 1,313-diazetidin-2-ylidine, singlet, trans $\left(\mathrm{C}_{1} / \mathrm{C}_{2}\right)^{\mathrm{t}}-187.720838$

$-187.751699$

12. singlet, cis, ts $\left(\mathrm{C}_{1} / \mathrm{C}_{\mathrm{s}}\right)^{\mathrm{t}, \mathrm{w}}$

$-187.720976$

$-187.751203$

12. triplet, trans $\left(\mathrm{C}_{2}\right)^{\mathrm{t}}$

$-187.620713$

$-187.651523$

12-C $\mathbf{H}^{+}$, trans $\left(\mathrm{C}_{1} \mathrm{C}_{2}\right)^{\mathrm{t}}$

$-188.113494$

12- $\mathrm{CH}_{2}$, cis, $\left(\mathrm{C}_{\mathrm{s}}\right)^{\mathrm{t}, \mathrm{x}}$

$-188.949105$

$-188.144773$

$-188.979806$

Set 13, 4-membered ring, $\mathrm{X}=\mathrm{NH}, \mathrm{Y}=\mathrm{CH}$ or $\mathrm{CH}_{2}$

13. azet-2-ylidene, singlet $\left(\mathrm{C}_{1}\right)^{\mathrm{y}}$

13. triplet $\left(\mathrm{C}_{1} / \mathrm{C}_{\mathrm{s}}\right)$

13- $\mathbf{C H}^{+}\left(\mathrm{C}_{1} / \mathrm{C}_{\mathrm{s}}\right)$

13- $\mathrm{CH}_{2}\left(\mathrm{C}_{1}\right)^{\mathrm{y}}$

13H $\mathrm{H}_{2}$ azetidin-2-ylidene, singlet $\left(\mathrm{C}_{1} / \mathrm{C}_{\mathrm{s}}\right)$

$\mathbf{1 3} \mathbf{H}_{2}$ triplet $\left(\mathrm{C}_{1}\right)^{\mathrm{y}}$

$13 \mathrm{H}_{2}-\mathbf{C H}^{+}\left(\mathrm{C}_{1} / \mathrm{C}_{\mathrm{s}}\right)$

$13 \mathrm{H}_{2}-\mathrm{CH}_{2}\left(\mathrm{C}_{1}\right)^{\mathrm{y}}$
$-170.423677$

$-170.361113$

$-170.787658$

$-171.703968$

$-171.665555$

$-171.590799$

$-172.063261$

$-172.917912$
$-170.453310$

$-170.392383$

$-170.817874$

$-171.733986$

$-171.695976$

$-171.622536$

$-172.093642$

$-172.948878$

Set 14, 5-membered ring, $X=\mathrm{NH}, \mathrm{Y}=\mathrm{CH}$

14. imidazole-5-ylidene, singlet $\left(\mathrm{C}_{\mathrm{s}}\right)$

14. triplet $\left(\mathrm{C}_{1}\right)$

14- $\mathbf{C H}^{+}$(imidazolium $\left.{ }^{+}\right)\left(\mathrm{C}_{2 \mathrm{v}}\right)$

14- $\mathrm{CH}_{2}\left(\mathrm{C}_{1}\right)$
$-225.789219$

$-225.685194$

$-226.218697$

$-226.961892$
$-225.820249$

$-225.718225$

$-226.249074$

$-226.994637$

${ }^{\mathrm{a}}$ In hartrees; 1 hartree $=627.51 \mathrm{kcal} / \mathrm{mol} .{ }^{\mathrm{b}} \mathrm{A}$ slash indicates the structure has virtually the higher symmetry. ${ }^{\mathrm{c}}$ The descriptors cis and trans refer to the two $\mathrm{N}-\mathrm{H}$ bonds. The triplet ts has iv $=285$ $\mathrm{cm}^{-1}$ corresponding to planarization of the ring (wobbling about the H-N-C-N-H moiety). ${ }^{\mathrm{d}}$ Protonation at $\mathrm{C} 2$ of the carbene gives the same conjugate acid as protonation at $\mathrm{N} 1$ of imidazole, namely, the imidazolium ion. The descriptors cis and trans refer to the two N-H 
bonds. The ring skeletons have an envelope shape. ${ }^{\mathrm{e}}$ The torsional angle about the $\mathrm{C} 4-\mathrm{C} 5$ bond is $21.5^{\circ}$. ${ }^{\mathrm{f}}$ This ts, iv $=98 \mathrm{~cm}^{-1}$, involves torsion about the C4-C5 bond. ${ }^{\mathrm{g}}$ The descriptors cis and trans refer to the two N-H bonds. The ring skeleton of the cis isomer has an envelope shape. The torsional angle about the $\mathrm{C} 4-\mathrm{C} 5$ bond in the trans isomer is $38.4^{\mathrm{o}}$. ${ }^{\mathrm{h}}$ Protonation at oxygen results in $\mathrm{C}-\mathrm{O}$ cleavage giving $(\mathrm{HO}-\mathrm{CH}=\mathrm{CH}-\mathrm{C} \equiv \mathrm{O})^{+}$in which the separated $\mathrm{C}$ and $\mathrm{O}$ are $2.600 \AA$ apart. ${ }^{\mathrm{i}}$ The stable form has an envelope-shaped ring skeleton. The ts is planar, $\mathrm{iv}=110 \mathrm{~cm}^{-1}$. ${ }^{\mathrm{j}}$ The torsional angle about $\mathrm{C} 4-\mathrm{C} 5$ is $40.5^{\circ}$. ${ }^{\mathrm{k}}$ The oxygen-protonated conjugate acid did not converge. It was undergoing fragmentation to a complex between protonated acetalehyde and carbon monoxide. 'The $\mathrm{C} 2-\mathrm{O}$ bond is somewhat elongated at $1.481 \AA$ while the $\mathrm{C} 2=\mathrm{N}$ bond is short at $1.323 \AA$. ${ }^{\mathrm{m}}$ The ring skeleton has an envelope shape with the $\mathrm{CH}_{2}$ unit out of the plane. ${ }^{\mathrm{n}}$ The torsional angle about $\mathrm{C} 4-\mathrm{C} 5$ is $12.8^{\circ}$. ${ }^{\circ}$ The ring skeleton has the envelope shape with $\mathrm{C} 5$ out of the plane. The $\mathrm{C} 2-\mathrm{O}$ bond is elongated to $1.520 \AA$ and the $\mathrm{C} 2=\mathrm{N}$ is short at $1.299 \AA .{ }^{\mathrm{p}} \mathrm{The}$ ring is completely buckled. The torsional angle about $\mathrm{C} 4-\mathrm{C} 5$ is $15.4^{\mathrm{o}}$. ${ }^{\mathrm{q}} \mathrm{The}$ descriptors cis and trans refer to the two P-H bonds. In series $\mathbf{4}$ and $\mathbf{4} \mathbf{H}_{\mathbf{2}}$ the phosphorus atoms are pyramidal, more so in the triplet than the singlet carbenes. The carbenium ions also have pyramidal phosphorus atoms, and those in the hydrogenation products are most pyramidal of all with bond angles less than $100^{\circ}$. The cis carbenium ion, $4-\mathbf{C H}^{+}$, is a transition state (ts), $\mathrm{i} v=360 \mathrm{~cm}^{-1}$, inversion about phosphorus, $\Delta H^{\ddagger}=4.1 \mathrm{kcal} / \mathrm{mol}$ (from the trans isomer). ${ }^{\mathrm{r}}$ The two phosphorus atoms in these species are not identical. For the singlet, cis carbene and the cis carbenium ion (ts) one phosphorus is virtually planar, the other decisively pyramidal. In the others both phosphorus

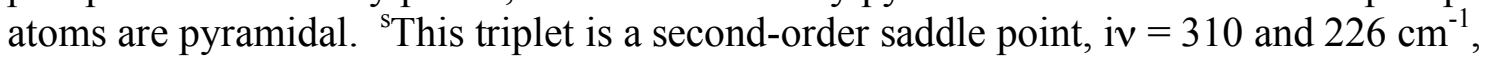
corresponding, respectively, to symmetric and asymmetric, pyramidalization at the nitrogens. Its $\mathrm{C} 4-\mathrm{N}$ and $\mathrm{C} 5-\mathrm{N}$ bonds are short at $1.318 \AA$, and the $\mathrm{C} 2-\mathrm{N}$ bonds long at $1.598 \AA$. 'The nitrogens are pyramidal in these species; cis and trans refer to the orientation of the N-H bonds. In the singlet the carbenic angle is $65.8^{\circ}$, and the N-N distance is $1.508 \AA$. In the triplet these values are $71.6^{\circ}$ and $1.629 \AA$. " Transition state for flattening the nitrogen valences; $i v=1313$, $1099 \mathrm{~cm}^{-1}$, corresponding to asymmetric and symmetric inversion at the nitrogens, respectively. ${ }^{\mathrm{v}}$ A stable structure at $\mathrm{HF} / 6-31 \mathrm{G}^{*}$, but a transition state for disrotatory ring opening/closing at the MP2 level of theory. The term exo, exo indicates that the coplanar $\mathrm{N}-\mathrm{H}$ bonds are directed away from each other. ${ }^{\mathrm{w}}$ Transition state for inversion at one nitrogen, $\mathrm{iv}=260 \mathrm{~cm}^{-1}$. The ring is folded. ${ }^{\mathrm{x}}$ The ring is folded, and the N-H hydrogens are in pseudo-equatorial positions. ${ }^{\mathrm{y}}$ The ring is folded and the nitrogen is pyramidal. 
Table S5. G3MP2 cartesian coordinates for compounds in this study: 91 sets of singlet and tripet carbenes, their conjugate acid carbenium ions, and their hydrogenation products.

\section{miscellaneous carbene sets}

carbene, singlet

$\begin{array}{rrrr}\mathrm{H} & 0.000000 & 0.862238 & -0.523024 \\ \mathrm{C} & 0.000000 & 0.000000 & 0.174341 \\ \mathrm{H} & 0.000000 & -0.862238 & -0.523024\end{array}$

carbene, triplet

$\begin{array}{rrrr}\mathrm{H} & 0.000000 & 0.982420 & -0.331211 \\ \mathrm{C} & 0.000000 & 0.000000 & 0.110404 \\ \mathrm{H} & 0.000000 & -0.982420 & -0.331211\end{array}$

methylt

$\begin{array}{cccc}\mathrm{H} & 0.000000 & 1.088256 & 0.000000 \\ \mathrm{C} & 0.000000 & 0.000000 & 0.000000 \\ \mathrm{H} & 0.942457 & -0.544128 & 0.000000 \\ \mathrm{H} & -0.942457 & -0.544128 & 0.000000\end{array}$

methane

$\begin{array}{cccc}\mathrm{H} & 0.628989 & 0.628989 & 0.628989 \\ \mathrm{C} & 0.000000 & 0.000000 & 0.000000 \\ \mathrm{H} & -0.628989 & -0.628989 & 0.628989 \\ \mathrm{H} & 0.628989 & -0.628989 & -0.628989 \\ \mathrm{H} & -0.628989 & 0.628989 & -0.628989\end{array}$

methylcarbene, singlet

H $0.642644 \quad 0.084212$

C $0.594625 \quad 0.021248$

1.088060

$\mathrm{H} 1.028370 \quad 0.953400$

$-0.026953$

C -0.854314

$-0.190034$

$-0.407828$

H 1.192475

$-0.847522$

$-0.035579$

$\mathrm{H}-1.305352$

0.822627

$-0.305958$

methylcarbene, triplet
$\mathrm{H} 0.453670 \quad-1.023029$
0.885452
C $-0.049474 \quad-0.615418$
0.000000
$\mathrm{H} 0.453670$
$-1.023029$
$-0.885452$
C -0.049474
0.859926
0.000000
$\mathrm{H}-1.073573$
$-0.997648$
0.000000
$\mathrm{H} \quad 0.759921$
1.576657
0.000000

ethylt, symmetrically H-bridged ion
$\mathrm{H} 0.000002$
0.000000
1.044690
C -0.690209
0.000000
$-0.062470$ 


$\begin{array}{lccc}\mathrm{H} & -1.244834 & -0.935696 & -0.073763 \\ \mathrm{C} & 0.690208 & 0.000000 & -0.062469 \\ \mathrm{H} & -1.244834 & 0.935696 & -0.073763 \\ \mathrm{H} & 1.244834 & -0.935696 & -0.073764 \\ \mathrm{H} & 1.244834 & 0.935696 & -0.073764 \\ & & \\ \text { ethane } \\ \mathrm{H} \quad 0.000000 & 1.018689 & 1.157275 \\ \mathrm{C} & 0.000000 & 0.000000 & 0.762189 \\ \mathrm{H} & 0.882211 & -0.509345 & 1.157275 \\ \mathrm{C} & 0.000000 & 0.000000 & -0.762189 \\ \mathrm{H}-0.882211 & -0.509345 & 1.157275 \\ \mathrm{H} & 0.882211 & 0.509345 & -1.157275 \\ \mathrm{H}-0.882211 & 0.509345 & -1.157275 \\ \mathrm{H} & 0.000000 & -1.018689 & -1.157275\end{array}$

dimethylcarbene, singlet

$\begin{array}{lrrr}\text { H } & -0.876222 & 1.214375 & -0.816643 \\ \text { C } & 0.000000 & 1.220860 & -0.149777 \\ \text { H } & 0.876222 & 1.214375 & -0.816643 \\ \text { C } & 0.000000 & 0.000000 & 0.703596 \\ \text { H } & 0.000000 & 2.150168 & 0.421162 \\ \text { C } & 0.000000 & -1.220860 & -0.149777 \\ \text { H } & 0.876222 & -1.214375 & -0.816643 \\ \text { H } & -0.876222 & -1.214375 & -0.816643 \\ \text { H } & 0.000000 & -2.150168 & 0.421162\end{array}$

dimethylcarbene, triplet

$\begin{array}{cccc}\mathrm{H} & 1.496391 & -0.744967 & -0.885353 \\ \mathrm{C} & 1.341346 & -0.113076 & 0.000000 \\ \mathrm{H} & 1.496391 & -0.744967 & 0.885353 \\ \mathrm{C} & 0.000000 & 0.505866 & 0.000000 \\ \mathrm{H} & 2.123105 & 0.650792 & 0.000000 \\ \mathrm{C} & -1.341346 & -0.113076 & 0.000000 \\ \mathrm{H} & -1.496391 & -0.744966 & 0.885354 \\ \mathrm{H} & -1.496391 & -0.744968 & -0.885353 \\ \mathrm{H} & -2.123105 & 0.650792 & -0.000001\end{array}$

isopropyl+

$\begin{array}{llll}\mathrm{H} & 2.035511 & 0.348745 & -0.591284\end{array}$

C $1.277159 \quad-0.196331 \quad-0.021389$

$\mathrm{H} 1.241144-1.263120 \quad-0.238951$

C $0.000002 \quad 0.462386 \quad-0.000038$

$\mathrm{H} 1.596946 \quad-0.072394 \quad 1.042106$

C $-1.277180 \quad-0.196286 \quad 0.021396$

$\mathrm{H} 0.000066 \quad 1.555072 \quad-0.000030$

$\mathrm{H}-1.241120 \quad-1.263047 \quad 0.239162$

$\mathrm{H}-1.596964 \quad-0.072717 \quad-1.042125$ 


\begin{tabular}{|c|c|c|c|}
\hline $\mathrm{H}$ & -2.035472 & 0.348844 & 0.591308 \\
\hline \multicolumn{4}{|c|}{ propane } \\
\hline $\mathrm{H}$ & -1.303387 & -0.904599 & 0.883524 \\
\hline C & -1.266727 & -0.260173 & 0.000000 \\
\hline $\mathrm{H}$ & -1.303321 & -0.904699 & -0.883454 \\
\hline $\mathrm{C}$ & 0.000000 & 0.587968 & 0.000000 \\
\hline $\mathrm{H}$ & -2.166508 & 0.361265 & -0.000069 \\
\hline $\mathrm{C}$ & 1.266727 & -0.260173 & 0.000000 \\
\hline $\mathrm{H}$ & 0.000000 & 1.245164 & 0.876738 \\
\hline $\mathrm{H}$ & 0.000000 & 1.245167 & -0.876737 \\
\hline $\mathrm{H}$ & 1.303320 & -0.904701 & -0.883452 \\
\hline $\mathrm{H}$ & 1.303388 & -0.904597 & 0.883525 \\
\hline $\mathrm{H}$ & 2.166508 & 0.361265 & -0.000071 \\
\hline
\end{tabular}

isopropylcarbene, singlet, (2-methylpropylidene)

$\begin{array}{llcc}\mathrm{C} & -0.124061 & -0.184920 & 0.429721 \\ \mathrm{C} & 0.739663 & -1.088262 & -0.316389 \\ \mathrm{H} & 1.547355 & -1.392571 & 0.377703 \\ \mathrm{C} & -1.506809 & 0.086264 & -0.126348 \\ \mathrm{C} & 0.965313 & 0.843751 & -0.062807 \\ \mathrm{H} & -0.113954 & -0.208386 & 1.524952 \\ \mathrm{H} & 0.487005 & 1.587855 & -0.696427 \\ \mathrm{H} & 1.717886 & 0.359808 & -0.728306 \\ \mathrm{H} & 1.484793 & 1.279773 & 0.787763 \\ \mathrm{H} & -2.194427 & -0.712375 & 0.165367 \\ \mathrm{H} & -1.461869 & 0.110375 & -1.217714 \\ \mathrm{H} & -1.911426 & 1.034525 & 0.241599\end{array}$

isopropylcarbene, triplet, ( 2 , methylpropylidene)

$\begin{array}{llll}\text { C } 0.010431 & 0.128874 & -0.346649\end{array}$

$\begin{array}{lll}\text { C } 0.373358 & 1.470006 & 0.167274\end{array}$

$\mathrm{H} \quad 1.296144 \quad 2.035075 \quad 0.110550$

C $-1.401057 \quad-0.259951 \quad 0.099481$

C $1.032463 \quad-0.927800 \quad 0.092901$

$\mathrm{H} 0.016276 \quad 0.164119 \quad-1.448166$

$\mathrm{H} 1.042910 \quad-1.013801 \quad 1.182930$

$\mathrm{H} 2.041215 \quad-0.666957-0.238800$

$\mathrm{H} \quad 0.777141-1.905147 \quad-0.328367$

$\mathrm{H}-2.136337 \quad 0.477568 \quad-0.231363$

$\mathrm{H}-1.450161 \quad-0.324173 \quad 1.190044$

$\mathrm{H}-1.678362-1.233451 \quad-0.314866$

isobutyl+, NA, structure rearranges to t-butyl+

isobutane
$\mathrm{H}-0.885472$
$1.984741 \quad 0.261334$
C 0.000000
$1.450293-0.096216$ 


$\begin{array}{llcc}\mathrm{H} & 0.000000 & 1.494137 & -1.190832 \\ \mathrm{C} & 0.000000 & 0.000000 & 0.376871 \\ \mathrm{H} & 0.885472 & 1.984741 & 0.261334 \\ \mathrm{C} & -1.255990 & -0.725146 & -0.096216 \\ \mathrm{H} & 0.000000 & 0.000000 & 1.475163 \\ \mathrm{C} & 1.255990 & -0.725146 & -0.096216 \\ \mathrm{H} & -1.293960 & -0.747068 & -1.190832 \\ \mathrm{H} & -2.161572 & -0.225529 & 0.261334 \\ \mathrm{H} & -1.276100 & -1.759212 & 0.261334 \\ \mathrm{H} & 1.293960 & -0.747068 & -1.190832 \\ \mathrm{H} & 1.276100 & -1.759212 & 0.261334 \\ \mathrm{H} & 2.161572 & -0.225529 & 0.261334\end{array}$

cyclopropanylcarbene, singlet, anti
$\mathrm{H}-0.639338$
1.247946
$-1.057633$
C -0.845480
0.724720
$-0.133530$
C -0.846369
$-0.723962$
$-0.134221$
C 0.394724
$-0.000949$
0.487663
$\mathrm{H}-1.437864$
1.258215
0.601071
$\mathrm{H}-0.640946$
$-1.246469$
$-1.058898$
$\mathrm{H}-1.439727$
$-1.257333$
0.599685
C 1.517839
$-0.000053$
$-0.372951$
$\mathrm{H} 0.401706$
$-0.001357$
1.578584
H 2.431883
0.000463
0.255426

cyclopropanylcarbene, singlet, syn
$\mathrm{H}-0.094785$
1.517542
$-0.698598$
C -0.476403
0.864144
0.077989
C -1.006708
$-0.471562$
$-0.294331$
C 0.309268
$-0.463793$
0.440409
$\mathrm{H}-0.847992$
1.378264
0.955896
$\mathrm{H}-0.980108$
$-0.733700$
$-1.347339$
H -1.864505
$-0.846742$
0.255892
C 1.534147
$-0.039856$
$-0.131636$
$\mathrm{H} 0.268325$
$-0.683936$
1.505103
H 1.357239
0.034976
$-1.225536$

cyclopropanylcarbene, singlet, rot. Ts
$\mathrm{H}-0.731103$
1.315666
$-1.027223$
C -0.826835
0.785742
$-0.083754$
C -0.880887
$-0.733425$
$-0.141874$
C 0.338424
$-0.037959$
0.375492
$\mathrm{H}-1.446582$
1.290146
0.650908
$\mathrm{H}-0.806932$
$-1.197592$
$-1.120337$
$\mathrm{H}-1.533719$
$-1.251318$
0.552634
C 1.695024
$-0.166870$
$-0.179199$
$\mathrm{H} 0.502003$
$-0.112294$
1.461991
H 2.061982
0.870460
$-0.341961$ 


\begin{tabular}{|c|c|c|c|}
\hline $\mathrm{H}$ & -0.572226 & 1.322255 & -1.030708 \\
\hline & -0.788925 & 0.788272 & -0.112369 \\
\hline & -0.887375 & -0.700990 & -0.173393 \\
\hline & 0.327005 & -0.065746 & 0.467447 \\
\hline & -1.422254 & 1.322939 & 0.587499 \\
\hline & -0.738545 & -1.180153 & -1.134375 \\
\hline & -1.594479 & -1.201506 & 0.478838 \\
\hline & 1.610290 & -0.142253 & -0.205113 \\
\hline & 0.350323 & -0.117574 & 1.556242 \\
\hline & 2.411210 & 0.578336 & -0.316932 \\
\hline
\end{tabular}

cyclopropylcarbinylt, bisected

$\begin{array}{llcc}\mathrm{H} & 1.451712 & 1.259082 & 0.641677 \\ \mathrm{C} & 0.945183 & 0.707569 & -0.145089 \\ \mathrm{C} & 0.945303 & -0.707284 & -0.145738 \\ \mathrm{C} & -0.375335 & -0.000488 & 0.535973 \\ \mathrm{H} & 0.714559 & 1.258720 & -1.049986 \\ \mathrm{H} & 1.452184 & -1.259352 & 0.640407 \\ \mathrm{H} & 0.714899 & -1.257581 & -1.051205 \\ \mathrm{H} & -0.393402 & -0.001006 & 1.618841 \\ \mathrm{C} & -1.509796 & 0.000115 & -0.206528 \\ \mathrm{H} & -1.481250 & 0.000710 & -1.294107 \\ \mathrm{H} & -2.490833 & -0.000038 & 0.262660\end{array}$

\begin{tabular}{lcc}
\multicolumn{3}{l}{ cyclopropylcarbinylt, perp ts } \\
H 1.419593 & 1.236784 & 0.819703 \\
C 0.930029 & 0.778519 & -0.034179 \\
C 1.000871 & -0.739268 & -0.113946 \\
C -0.282062 & -0.020070 & 0.206171 \\
H 0.968362 & 1.350535 & -0.956011 \\
H 1.506951 & -1.261205 & 0.690274 \\
H 1.035424 & -1.224616 & -1.084199 \\
H -0.703481 & -0.241866 & 1.266646 \\
C -1.624262 & 0.000637 & -0.120639 \\
H -2.213718 & -0.916318 & -0.077914 \\
H -2.160588 & 0.937776 & -0.282942
\end{tabular}

methylcyclopropane

$\begin{array}{llcc}\mathrm{H} & 0.745647 & -1.258665 & -1.079421 \\ \mathrm{C} & 0.950079 & -0.752582 & -0.140984 \\ \mathrm{C} & 0.950074 & 0.752585 & -0.140982 \\ \mathrm{C} & -0.179383 & -0.000003 & 0.501601 \\ \mathrm{H} & 1.662291 & -1.266500 & 0.495719 \\ \mathrm{H} & 0.745637 & 1.258670 & -1.079417 \\ \mathrm{H} & 1.662283 & 1.266506 & 0.495722 \\ \mathrm{H} & -0.184313 & -0.000006 & 1.589208\end{array}$




\begin{tabular}{|c|c|c|c|}
\hline $\mathrm{C}$ & -1.544296 & 0.000000 & -0.136256 \\
\hline $\mathrm{H}$ & -2.117818 & -0.886251 & 0.152567 \\
\hline $\mathrm{H}$ & -1.454821 & -0.000085 & -1.227087 \\
\hline $\mathrm{H}$ & -2.117755 & 0.886334 & 0.152436 \\
\hline \multicolumn{4}{|c|}{ cyclohexa-2,4-dienylidene, singlet } \\
\hline $\mathrm{C}$ & 1.163881 & -0.761271 & 0.000093 \\
\hline $\mathrm{C}$ & -0.095707 & -1.556688 & -0.000025 \\
\hline $\mathrm{C}$ & -1.254937 & -0.718596 & -0.000118 \\
\hline $\mathrm{C}$ & -1.215611 & 0.662440 & 0.000097 \\
\hline $\mathrm{H}$ & -2.237679 & -1.189812 & -0.000262 \\
\hline $\mathrm{H}$ & -2.149938 & 1.225582 & 0.000382 \\
\hline C & 0.009862 & 1.403217 & -0.000002 \\
\hline C & 1.183470 & 0.721883 & -0.000147 \\
\hline $\mathrm{H}$ & -0.018537 & 2.489144 & -0.000030 \\
\hline $\mathrm{H}$ & 2.137201 & 1.247876 & -0.000180 \\
\hline $\mathrm{H}$ & 1.761359 & -1.139195 & 0.850561 \\
\hline $\mathrm{H}$ & 1.761846 & -1.139513 & -0.849860 \\
\hline \multicolumn{4}{|c|}{ cyclohexa-2,4-dienylidene-to-benzene, singlet, ts } \\
\hline $\mathrm{C}$ & 1.098249 & -0.835783 & 0.020484 \\
\hline $\mathrm{C}$ & -0.193878 & -1.547305 & -0.092626 \\
\hline $\mathrm{C}$ & -1.287059 & -0.617515 & -0.026475 \\
\hline $\mathrm{C}$ & -1.151575 & 0.757609 & 0.033277 \\
\hline $\mathrm{H}$ & -2.298599 & -1.020236 & -0.073466 \\
\hline $\mathrm{H}$ & -2.040740 & 1.385768 & 0.087878 \\
\hline $\mathrm{C}$ & 0.122105 & 1.391408 & 0.004372 \\
\hline $\mathrm{C}$ & 1.245393 & 0.618091 & -0.059938 \\
\hline $\mathrm{H}$ & 0.185469 & 2.475962 & -0.013477 \\
\hline $\mathrm{H}$ & 2.234193 & 1.067593 & -0.126064 \\
\hline $\mathrm{H}$ & 0.938858 & -1.096854 & 1.115790 \\
\hline $\mathrm{H}$ & 1.981411 & -1.411261 & -0.265227 \\
\hline \multicolumn{4}{|c|}{ cyclohexa-2,4-dienylidene, } \\
\hline $\mathrm{H}$ & -1.975935 & -0.887827 & 0.872969 \\
\hline C & -1.356748 & -0.625949 & 0.000005 \\
\hline $\mathrm{H}$ & -1.975957 & -0.887837 & -0.872940 \\
\hline C & -0.092043 & -1.393933 & -0.000006 \\
\hline $\mathrm{C}$ & 1.161552 & -0.909340 & -0.000001 \\
\hline $\mathrm{C}$ & 1.340794 & 0.488063 & 0.000002 \\
\hline $\mathrm{H}$ & 2.022613 & -1.571947 & 0.000001 \\
\hline $\mathrm{C}$ & 0.193784 & 1.336783 & 0.000000 \\
\hline $\mathrm{H}$ & 2.337493 & 0.915218 & 0.000005 \\
\hline C & -1.055418 & 0.853080 & -0.000005 \\
\hline $\mathrm{H}$ & 0.347782 & 2.412576 & 0.000005 \\
\hline $\mathrm{H}$ & -1.907524 & 1.527594 & -0.000011 \\
\hline
\end{tabular}




$\begin{array}{lcr}\text { cyclohexa-2, } & \text {-dienylidene, singlet } \\ \text { C } 0.000000 & 0.000000 & 1.588386 \\ \text { C } 0.000000 & 1.208191 & 0.784522 \\ \text { C } 0.000000 & 1.242927 & -0.578007 \\ \text { H } 0.000000 & 2.162480 & 1.311476 \\ \text { H } 0.000000 & 2.188652 & -1.120614 \\ \text { C } 0.000000 & 0.000000 & -1.378229 \\ \text { H } 0.866603 & 0.000000 & -2.060420 \\ \text { H }-0.866603 & 0.000000 & -2.060420 \\ \text { C } 0.000000 & -1.242927 & -0.578007 \\ \text { C } 0.000000 & -1.208191 & 0.784522 \\ \text { H } 0.000000 & -2.188652 & -1.120614 \\ \text { H } 0.000000 & -2.162480 & 1.311476\end{array}$

cyclohexa-2,5-dienylidene, triplet

$\begin{array}{llcr}\mathrm{C} & 0.000000 & 0.000000 & 1.459911 \\ \mathrm{C} & 0.000000 & 1.241963 & 0.796252 \\ \mathrm{C} & 0.000000 & 1.257142 & -0.547611 \\ \mathrm{H} & 0.000000 & 2.167630 & 1.364327 \\ \mathrm{H} & 0.000000 & 2.202718 & -1.083394 \\ \mathrm{C} & 0.000000 & 0.000000 & -1.367965 \\ \mathrm{H} & 0.868240 & 0.000000 & -2.048619 \\ \mathrm{H} & -0.868240 & 0.000000 & -2.048619 \\ \mathrm{C} & 0.000000 & -1.257142 & -0.547611 \\ \mathrm{C} & 0.000000 & -1.241963 & 0.796252 \\ \mathrm{H} & 0.000000 & -2.202718 & -1.083394 \\ \mathrm{H} & 0.000000 & -2.167630 & 1.364327\end{array}$

benzenonium+

$\begin{array}{cccc}\mathrm{H} & 0.853560 & 0.000000 & -2.102107 \\ \mathrm{C} & 0.000000 & 0.000000 & -1.393602 \\ \mathrm{H} & -0.853560 & 0.000000 & -2.102107 \\ \mathrm{C} & 0.000000 & 1.250301 & -0.632191 \\ \mathrm{C} & 0.000000 & -1.250301 & -0.632191 \\ \mathrm{H} & 0.000000 & -2.189348 & -1.180801 \\ \mathrm{C} & 0.000000 & -1.237553 & 0.742519 \\ \mathrm{H} & 0.000000 & -2.161791 & 1.311027 \\ \mathrm{C} & 0.000000 & 0.000000 & 1.413244 \\ \mathrm{C} & 0.000000 & 1.237553 & 0.742519 \\ \mathrm{H} & 0.000000 & 0.000000 & 2.501959 \\ \mathrm{H} & 0.000000 & 2.189348 & -1.180801 \\ \mathrm{H} & 0.000000 & 2.161791 & 1.311027\end{array}$

cyclohexa-1,3-diene

$\begin{array}{llll}\mathrm{H} & 0.019551 & 1.268477 & -2.048796 \\ \mathrm{C} & 0.341311 & 0.682964 & -1.181330 \\ \mathrm{H} & 1.429650 & 0.545383 & -1.278942 \\ \mathrm{C} & -0.341311 & -0.682964 & -1.181330\end{array}$




$\begin{array}{llcc}\mathrm{C} & 0.055589 & 1.417795 & 0.100698 \\ \mathrm{H} & -0.019551 & -1.268477 & -2.048796 \\ \mathrm{H}-1.429650 & -0.545383 & -1.278942 \\ \mathrm{C}-0.055589 & -1.417795 & 0.100698 \\ \mathrm{H} & 0.011201 & 2.504418 & 0.095651 \\ \mathrm{C} & 0.055589 & -0.728849 & 1.253342 \\ \mathrm{H}-0.011201 & -2.504418 & 0.095651 \\ \mathrm{C} & -0.055589 & 0.728849 & 1.253342 \\ \mathrm{H} & 0.219629 & -1.245752 & 2.195830 \\ \mathrm{H} & -0.219629 & 1.245752 & 2.195830 \\ & & & \\ \mathrm{CyClohexa-1}, 4-\mathrm{diene} & \\ \mathrm{C} & 0.000009 & -0.669330 & 1.248521 \\ \mathrm{C} & -0.000016 & -1.500332 & 0.000000 \\ \mathrm{H} & 0.000027 & -1.203071 & 2.197416 \\ \mathrm{H} & 0.871259 & -2.172454 & 0.000000 \\ \mathrm{H} & -0.871331 & -2.172403 & 0.000000 \\ \mathrm{C} & 0.000009 & 0.669330 & -1.248521 \\ \mathrm{C} & 0.000009 & -0.669330 & -1.248521 \\ \mathrm{H} & 0.000027 & 1.203071 & -2.197416 \\ \mathrm{H} & 0.000027 & -1.203071 & -2.197416 \\ \mathrm{C} & -0.000016 & 1.500332 & 0.000000 \\ \mathrm{C} & 0.000009 & 0.669330 & 1.248521 \\ \mathrm{H} & 0.000027 & 1.203071 & 2.197416 \\ \mathrm{H} & -0.871331 & 2.172403 & 0.000000 \\ \mathrm{H} & 0.871259 & 2.172454 & 0.000000\end{array}$

6,6-dimethylcyclohexa-2,4-dienylidene, singlet

$\begin{array}{lll}\text { C }-1.656446 & -0.039433 & -1.251767\end{array}$

C $-0.742311 \quad-0.034033 \quad-0.000070$

$\begin{array}{lll}\text { C } 0.036629 & 1.233215 & -0.000444\end{array}$

C $-0.042509 \quad-1.355875 \quad 0.000010$

C $-1.655417 \quad-0.039077 \quad 1.252419$

$\mathrm{H}-2.291132 \quad 0.852932 \quad 1.255581$

$\mathrm{H}-1.059669 \quad-0.046016 \quad 2.169320$

$\mathrm{H}-2.277732 \quad-0.935203 \quad 1.233121$

$\mathrm{H}-1.061475 \quad-0.046687 \quad-2.169171$

$\mathrm{H}-2.292111 \quad 0.852613 \quad-1.254674$

$\mathrm{H}-2.278819-0.935500-1.231701$

C $1.392911 \quad 1.218266 \quad-0.000154$

$\mathrm{H}-0.505158 \quad 2.180684 \quad-0.000900$

H $1.974076 \quad 2.136361 \quad-0.000229$

$\begin{array}{lll}\text { C } 2.063981 & -0.045886 & 0.000047\end{array}$

C $1.380218 \quad-1.245994 \quad-0.000262$

H $3.155112 \quad-0.046160 \quad 0.000359$

$\mathrm{H} 1.974566-2.160123 \quad-0.000380$ 


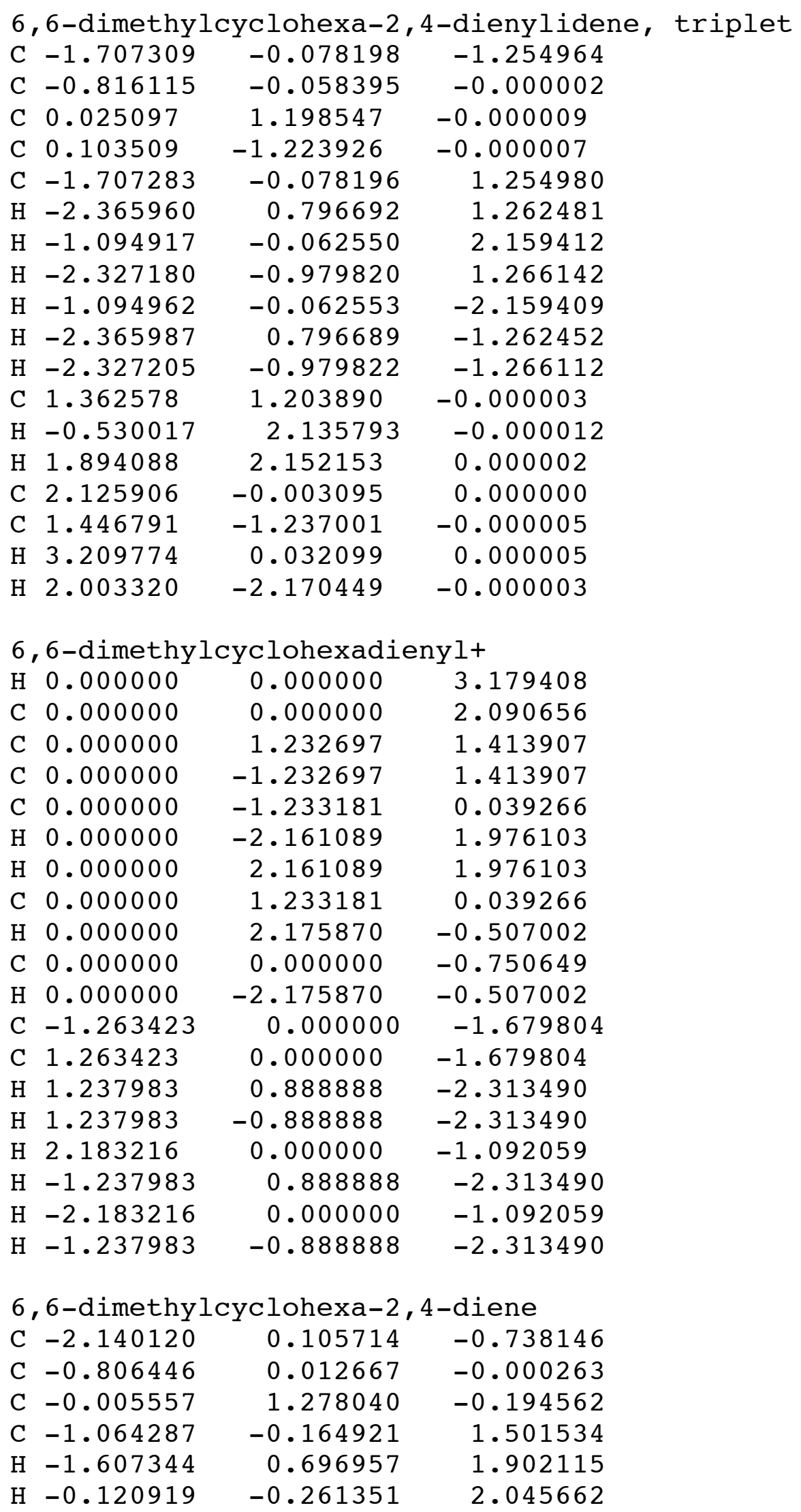




\begin{tabular}{|c|c|c|c|}
\hline $\mathrm{H}$ & -1.666250 & -1.063189 & 1.6 \\
\hline $\mathrm{H}$ & -1.983672 & 0.257786 & -1.810702 \\
\hline & -2.737616 & 0.941656 & -0.358564 \\
\hline & -2.721000 & -0.813126 & -0.602839 \\
\hline & 1.340931 & 1.270722 & -0. \\
\hline $\mathrm{H}$ & -0.548383 & 2.217320 & -0.300277 \\
\hline $\mathrm{H}$ & 1.902617 & 2.198552 & -0.213239 \\
\hline C & 2.066049 & 0.018805 & 0.066186 \\
\hline C & 1.431763 & -1.158552 & -0.095014 \\
\hline $\mathrm{H}$ & 3.117250 & 0.053222 & 0.342247 \\
\hline $\mathrm{H}$ & 1.964692 & -2.099400 & 0.023808 \\
\hline $\mathrm{C}$ & -0.002451 & -1.174980 & -0.544509 \\
\hline & -0.023277 & -1.142543 & -1.646550 \\
\hline & -0.495391 & -2.110855 & -0.252332 \\
\hline
\end{tabular}

\begin{tabular}{lcc}
\multicolumn{3}{l}{ spiro[2.5]octa-4,6-dien-8-ylidene, singlet } \\
C -1.346558 & 0.006843 & 0.000000 \\
C -0.041451 & 0.611724 & 0.000000 \\
C -1.241732 & -1.415381 & 0.000000 \\
C -0.040619 & -2.112144 & 0.000000 \\
H -2.158814 & -2.006215 & 0.000000 \\
C 1.210091 & -1.444431 & 0.000000 \\
H -0.045620 & -3.202869 & 0.000000 \\
C 1.223997 & -0.075470 & 0.000000 \\
H 2.135303 & -2.014339 & 0.000000 \\
H 2.164291 & 0.477593 & 0.000000 \\
C -0.040619 & 2.034459 & 0.718460 \\
C -0.040619 & 2.034459 & -0.718460 \\
H -0.971080 & 2.206442 & 1.243378 \\
H 0.876031 & 2.246300 & 1.257440 \\
H -0.971080 & 2.206442 & -1.243378 \\
H 0.876031 & 2.246300 & -1.257440
\end{tabular}

\begin{tabular}{|c|c|c|}
\hline$c-0.130470$ & -1.227687 & -0.000001 \\
\hline 0.718927 & -0.047606 & -0.000001 \\
\hline-1.475480 & -1.245874 & 0.000000 \\
\hline-2.159450 & -0.015917 & 0.000001 \\
\hline-2.027185 & -2.181807 & 0.000000 \\
\hline-1.411330 & 1.194883 & 0.000000 \\
\hline-3.243540 & 0.009953 & 0.000002 \\
\hline-0.069105 & 1.203335 & -0.000002 \\
\hline-1.949476 & 2.138948 & 0.000003 \\
\hline 0.483466 & 2.141080 & -0.000003 \\
\hline 2.053018 & -0.078097 & -0.743864 \\
\hline 2.053017 & -0.078096 & 0.743866 \\
\hline 2.294746 & -0.999617 & -1.262542 \\
\hline 2.336246 & 0.830703 & -1.265312 \\
\hline
\end{tabular}




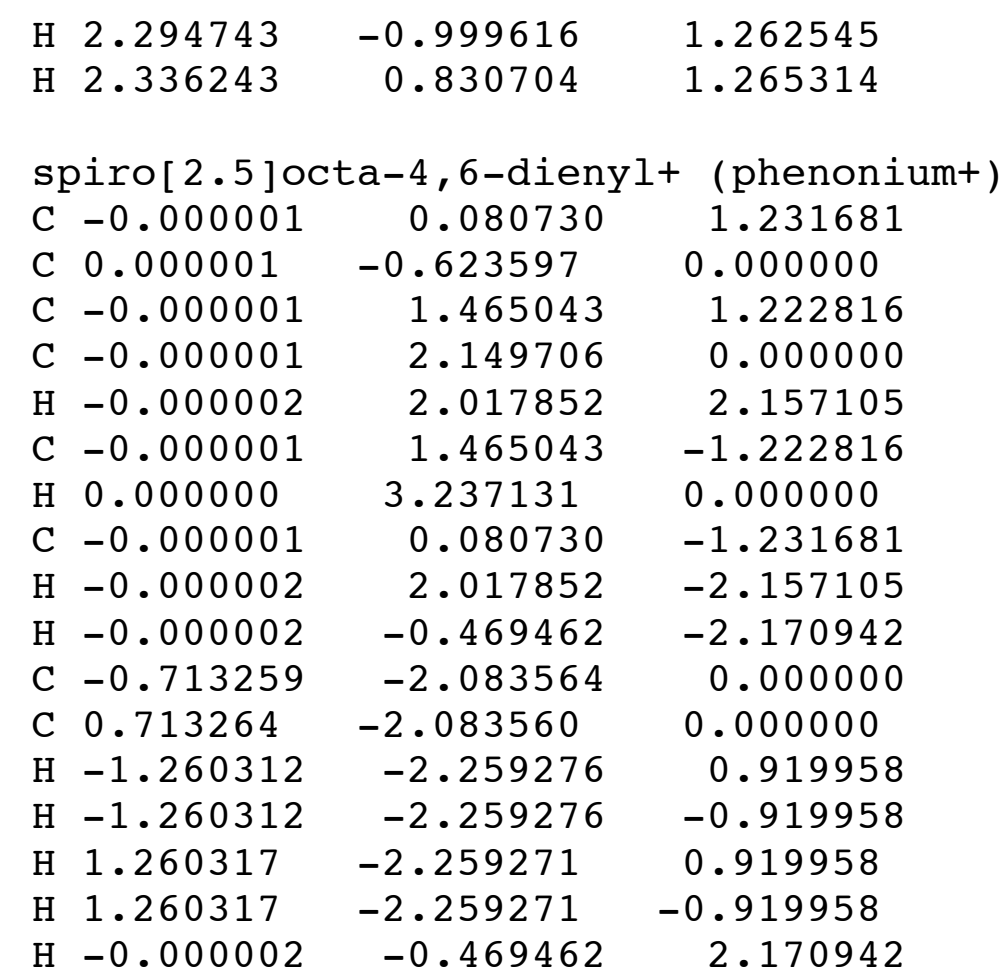

\begin{tabular}{|c|c|c|c|}
\hline $\mathrm{C}$ & 0.715180 & 0.028429 & -0.135903 \\
\hline $\mathrm{C}$ & -1.446355 & -1.178470 & 0.107839 \\
\hline C & -2.077003 & 0.002586 & 0.260299 \\
\hline $\mathrm{H}$ & -1.948773 & -2.112994 & 0.347326 \\
\hline $\mathrm{C}$ & -1.404741 & 1.254007 & -0.076085 \\
\hline $\mathrm{H}$ & -3.092498 & 0.040343 & 0.647502 \\
\hline $\mathrm{C}$ & -0.069373 & 1.277026 & -0.270862 \\
\hline $\mathrm{H}$ & -1.988425 & 2.169040 & -0.140956 \\
\hline $\mathrm{H}$ & 0.452674 & 2.212182 & -0.470161 \\
\hline $\mathrm{C}$ & 2.191686 & 0.052368 & -0.418906 \\
\hline C & 1.716823 & -0.038321 & 1.000752 \\
\hline $\mathrm{H}$ & 2.631147 & -0.831055 & -0.873155 \\
\hline $\mathrm{H}$ & 2.626013 & 0.989938 & -0.754024 \\
\hline $\mathrm{H}$ & 1.816053 & -0.988487 & 1.517371 \\
\hline $\mathrm{H}$ & 1.820276 & 0.834952 & 1.637085 \\
\hline C & -0.067008 & -1.217135 & -0.492970 \\
\hline $\mathrm{H}$ & 0.481919 & -2.108370 & -0.165900 \\
\hline $\mathrm{H}$ & -0.153635 & -1.288485 & -1.590077 \\
\hline
\end{tabular}

$\begin{array}{lcc}\text { spiro[2.5]octa-4,7-dien-6-ylidene, singlet } \\ \text { H } 1.261639 & 0.915554 & -2.229412 \\ \text { C } 0.730582 & 0.000000 & -1.994345 \\ \text { C } 0.000000 & 0.000000 & -0.621596 \\ \text { C }-0.730582 & 0.000000 & -1.994345 \\ \text { H } 1.261639 & -0.915554 & -2.229412\end{array}$




$\begin{array}{cccc}\mathrm{C} & 0.000000 & 1.231337 & 0.129774 \\ \mathrm{C} & 0.000000 & -1.231337 & 0.129774 \\ \mathrm{H} & -1.261639 & 0.915554 & -2.229412 \\ \mathrm{H} & -1.261639 & -0.915554 & -2.229412 \\ \mathrm{H} & 0.000000 & -2.172768 & -0.424416 \\ \mathrm{C} & 0.000000 & -1.199192 & 1.500625 \\ \mathrm{H} & 0.000000 & 2.172768 & -0.424416 \\ \mathrm{C} & 0.000000 & 1.199192 & 1.500625 \\ \mathrm{H} & 0.000000 & -2.159641 & 2.017972 \\ \mathrm{C} & 0.000000 & 0.000000 & 2.304577 \\ \mathrm{H} & 0.000000 & 2.159641 & 2.017972\end{array}$

spiro[2.5] octa-4,7-dien-6-ylidene, triplet

$\begin{array}{llcc}\mathrm{H} & 1.265246 & 0.913752 & -2.230004 \\ \mathrm{C} & 0.743374 & 0.000000 & -1.963623 \\ \mathrm{C} & 0.000000 & 0.000000 & -0.627488 \\ \mathrm{C} & -0.743374 & 0.000000 & -1.963623 \\ \mathrm{H} & 1.265246 & -0.913752 & -2.230004 \\ \mathrm{C} & 0.000000 & 1.248065 & 0.156069 \\ \mathrm{C} & 0.000000 & -1.248065 & 0.156069 \\ \mathrm{H} & -1.265246 & 0.913752 & -2.230004 \\ \mathrm{H} & -1.265246 & -0.913752 & -2.230004 \\ \mathrm{H} & 0.000000 & -2.188410 & -0.391744 \\ \mathrm{C} & 0.000000 & -1.238562 & 1.502466 \\ \mathrm{H} & 0.000000 & 2.188410 & -0.391744 \\ \mathrm{C} & 0.000000 & 1.238562 & 1.502466 \\ \mathrm{H} & 0.000000 & -2.167396 & 2.065259 \\ \mathrm{C} & 0.000000 & 0.000000 & 2.166495 \\ \mathrm{H} & 0.000000 & 2.167396 & 2.065259\end{array}$

\begin{tabular}{|c|c|c|c|}
\hline $\mathrm{H}$ & 2.355886 & -0.913054 & -1.264842 \\
\hline $\mathrm{C}$ & 2.078131 & 0.000001 & -0.747036 \\
\hline C & 0.756786 & 0.000000 & -0.000003 \\
\hline C & 2.078072 & -0.000001 & 0.747149 \\
\hline $\mathrm{H}$ & 2.355885 & 0.913058 & -1.264839 \\
\hline C & -0.039996 & -1.242979 & -0.000072 \\
\hline $\mathrm{C}$ & -0.039996 & 1.242979 & -0.000072 \\
\hline $\mathrm{H}$ & 2.355775 & -0.913059 & 1.264978 \\
\hline $\mathrm{H}$ & 2.355778 & 0.913055 & 1.264981 \\
\hline $\mathrm{H}$ & 0.507490 & -2.185715 & -0.000168 \\
\hline C & -1.380613 & -1.248742 & -0.000067 \\
\hline $\mathrm{H}$ & 0.507490 & 2.185716 & -0.000167 \\
\hline $\mathrm{C}$ & -1.380613 & 1.248742 & -0.000066 \\
\hline C & -2.212846 & 0.000000 & 0.000129 \\
\hline $\mathrm{H}$ & -1.910269 & -2.199710 & -0.000147 \\
\hline $\mathrm{H}$ & -1.910269 & 2.199709 & -0.000147 \\
\hline & -2.885453 & 0.000000 & 0.871596 \\
\hline
\end{tabular}




$$
\begin{array}{lcc}
\text { H }-2.885866 & 0.000000 & -0.871014 \\
\text { vinylcarbene, singlet, anti } \\
\text { H }-1.077205 & -1.281730 & -0.111567 \\
\text { C }-1.093479 & -0.205915 & 0.023792 \\
\text { C } 0.073914 & 0.484789 & -0.107881 \\
\text { H }-2.023971 & 0.263884 & 0.334094 \\
\text { C } 1.188882 & -0.394634 & -0.031676 \\
\text { H } 0.078351 & 1.573291 & -0.019971 \\
\text { H } 2.006925 & 0.139116 & 0.492035
\end{array}
$$

vinylcarbene, singlet, rotational ts

$\mathrm{H} 1.362268-1.245210 \quad-0.068062$

$\begin{array}{lll}\text { C } 1.186877 & -0.179082 & 0.012364\end{array}$

$\begin{array}{lll}\text { C }-0.054123 & 0.334989 & 0.012225\end{array}$

$\begin{array}{llll}\mathrm{H} & 2.052261 & 0.468311 & 0.060150\end{array}$

C $-1.370586 \quad-0.188876 \quad-0.166184$

$\mathrm{H}-0.180699 \quad 1.431117 \quad 0.041761$

$\mathrm{H}-1.806836 \quad-0.456409 \quad 0.815719$

$\begin{array}{lcc}\text { vinylcarbene, triplet, anti } \\ \text { H }-1.299176 & -1.255713 & 0.000000 \\ \text { C }-1.173304 & -0.180045 & 0.000000 \\ \text { C } 0.072259 & 0.386486 & -0.000001 \\ \text { H }-2.068013 & 0.428263 & 0.000001 \\ \text { C } 1.256224 & -0.311817 & 0.000000 \\ \text { H } 0.141030 & 1.476587 & 0.000001 \\ \text { H } 2.295090 & -0.016887 & 0.000002\end{array}$

$\begin{array}{lcc}\text { vinylcarbene, triplet, syn } \\ \text { H }-1.128553 & -1.325169 & -0.000005 \\ \text { C }-1.116526 & -0.241623 & -0.000001 \\ \text { C } 0.060569 & 0.459974 & 0.000001 \\ \text { H }-2.073126 & 0.264838 & 0.000005 \\ \text { C } 1.311292 & -0.110398 & -0.000003 \\ \text { H } 0.011516 & 1.547335 & 0.000001 \\ \text { H } 1.658155 & -1.134723 & 0.000015\end{array}$

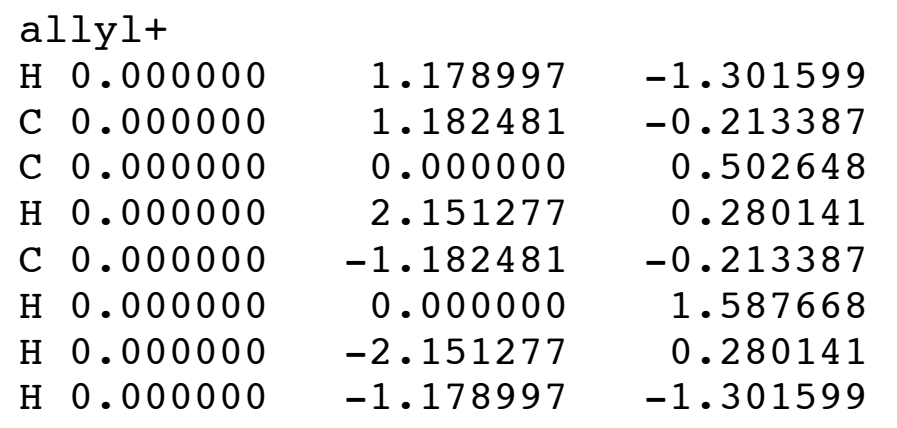




$\begin{array}{lcc}\text { propene } & & \\ \text { H } 1.805488 & 0.142331 & -0.879917 \\ \text { C } 1.230458 & -0.164128 & 0.000000 \\ \text { H } 1.159716 & -1.254923 & -0.000912 \\ \text { C }-0.130219 & 0.461369 & -0.000010 \\ \text { H } 1.804793 & 0.140898 & 0.880875 \\ \text { C }-1.278865 & -0.222075 & 0.000001 \\ \text { H }-0.164239 & 1.550111 & -0.000014 \\ \text { H }-1.293126 & -1.308306 & -0.000018 \\ \text { H }-2.240879 & 0.278891 & 0.000039\end{array}$

ethynylcarbene, singlet

\begin{tabular}{|c|c|c|}
\hline Н 2.306162 & 0.142479 & 0.000030 \\
\hline 1.251814 & -0.026425 & 0.000007 \\
\hline 0.016171 & 0.039806 & -0.000015 \\
\hline-1.337797 & -0.168152 & -0.000005 \\
\hline-1.887288 & 0.786145 & 0.000052 \\
\hline
\end{tabular}

ethynylcarbene, triplet

\begin{tabular}{|c|c|c|}
\hline H 2.297084 & 0.166347 & 0.129566 \\
\hline 1.263854 & -0.037212 & -0.039232 \\
\hline 0.000006 & 0.019048 & 0.000033 \\
\hline-1.263865 & -0.037375 & 0.039119 \\
\hline-2.297055 & 0.166890 & -0.129089 \\
\hline
\end{tabular}

ethynylCH2+ (propargyl+)

$\mathrm{H} 0.000000 \quad 0.000000 \quad 2.418196$

C $0.000000 \quad 0.000000 \quad 1.340013$

$\begin{array}{lll}\text { C } 0.000000 & 0.000000 & 0.108023\end{array}$

C $0.000000 \quad 0.000000 \quad-1.248665$

$\mathrm{H} 0.000000 \quad 0.936921 \quad-1.807213$

$\mathrm{H} 0.000000-0.936921 \quad-1.807213$

\begin{tabular}{|c|c|c|}
\hline & & \\
\hline $\mathrm{H} 0.000000$ & 0.000000 & 2.498858 \\
\hline 0.000000 & 0.000000 & 1.432831 \\
\hline 0.000000 & 0.000000 & 0.214800 \\
\hline 0.000000 & 0.000000 & -1.246063 \\
\hline 0.000000 & 1.021388 & -1.636088 \\
\hline-0.884548 & -0.510694 & -1.636088 \\
\hline 0.884548 & -0.510694 & -1.636088 \\
\hline
\end{tabular}

cyclopropanylidene, singlet
C 0.000000
0.000000
0.991622

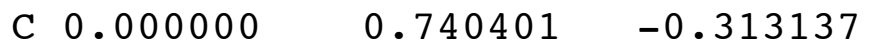
$\mathrm{H}-0.909176 \quad 1.289322 \quad-0.548022$
C $0.000000 \quad-0.740401 \quad-0.313137$
$\mathrm{H} 0.909176 \quad 1.289322 \quad-0.548022$ 


$\begin{array}{llll}\mathrm{H} & -0.909176 & -1.289322 & -0.548022 \\ \mathrm{H} & 0.909176 & -1.289322 & -0.548022\end{array}$

cyclopropanylidene, triplet

$\begin{array}{cccc}\text { C } & 0.000000 & 0.000000 & 0.938530 \\ \mathrm{C} & 0.000000 & 0.797518 & -0.264916 \\ \mathrm{H} & -0.913768 & 1.276696 & -0.613045 \\ \mathrm{C} & 0.000000 & -0.797518 & -0.264916 \\ \mathrm{H} & 0.913768 & 1.276696 & -0.613045 \\ \mathrm{H} & -0.913768 & -1.276696 & -0.613045 \\ \mathrm{H} & 0.913768 & -1.276696 & -0.613045\end{array}$

\begin{tabular}{|c|c|c|c|}
\hline \multicolumn{4}{|c|}{ cyclopropyl+ } \\
\hline $\mathrm{H}$ & -0.000480 & -1.301611 & 1.178409 \\
\hline C & -0.000032 & -0.213415 & 1.182348 \\
\hline C & -0.000032 & -0.213415 & -1.182348 \\
\hline $\mathrm{C}$ & -0.000032 & 0.502812 & 0.000000 \\
\hline $\mathrm{H}$ & 0.000601 & 0.279774 & 2.151284 \\
\hline $\mathrm{H}$ & -0.000480 & -1.301611 & -1.178409 \\
\hline $\mathrm{H}$ & 0.000601 & 0.279774 & -2.151284 \\
\hline & 0.000330 & 1.587783 & 0.000000 \\
\hline
\end{tabular}

cyclopropane

$\begin{array}{lllc}\mathrm{C} & 0.000000 & 0.866820 & 0.000000 \\ \mathrm{H} & 0.000000 & 1.455591 & 0.910104 \\ \mathrm{C}-0.750688 & -0.433410 & 0.000000 \\ \mathrm{H} & 0.000000 & 1.455591 & -0.910104 \\ \mathrm{H} & -1.260579 & -0.727796 & 0.910104 \\ \mathrm{H} & -1.260579 & -0.727796 & -0.910104 \\ \mathrm{C} & 0.750688 & -0.433410 & 0.000000 \\ \mathrm{H} & 1.260579 & -0.727796 & 0.910104 \\ \mathrm{H} & 1.260579 & -0.727796 & -0.910104\end{array}$

cyclobutanylidene, singlet
C 0.000000
$-0.812718$
$-0.481257$
C 1.122027
$-0.213450$
0.206680
H 1.205729
$-0.166180$
1.300880
C 0.000000
0.871391
$-0.183030$
H 2.085805
$-0.198229$
$-0.298662$
C -1.122027
$-0.213450$
0.206680
$\mathrm{H} 0.000000$
1.365163
$-1.146380$
$\mathrm{H} 0.000000$
1.573024
0.647499
$\mathrm{H}-1.205725$
$-0.166180$
1. 300880
H -2.085807
$-0.198229$
$-0.298659$

cyclobutanylidene, triplet
C 0.000000
$-1.117792$
$-0.000071$
C -1.129723
$-0.132052$
0.000033 


\begin{tabular}{|c|c|c|c|}
\hline $\mathrm{H}$ & -1.770855 & -0.131380 & -0.888165 \\
\hline $\mathrm{C}$ & 0.000000 & 0.944564 & -0.000030 \\
\hline $\mathrm{H}$ & -1.770683 & -0.131420 & 0.888358 \\
\hline $\mathrm{C}$ & 1.129723 & -0.132052 & 0.000033 \\
\hline $\mathrm{H}$ & 0.000000 & 1.574881 & 0.891444 \\
\hline $\mathrm{H}$ & 0.000000 & 1.574714 & -0.891619 \\
\hline $\mathrm{H}$ & 1.770855 & -0.131380 & -0.888165 \\
\hline $\mathrm{H}$ & 1.770683 & -0.131420 & 0.888358 \\
\hline \multicolumn{4}{|c|}{ cyclobutyl+ } \\
\hline $\mathrm{H}$ & -0.000058 & 1.647481 & 0.465302 \\
\hline C & 0.000093 & 0.864922 & -0.287993 \\
\hline C & -1.186112 & -0.143013 & 0.255860 \\
\hline C & 1.186069 & -0.143080 & 0.255907 \\
\hline C & -0.000078 & -0.782651 & -0.205882 \\
\hline $\mathrm{H}$ & -0.000095 & -1.226419 & -1.199799 \\
\hline $\mathrm{H}$ & 0.000157 & 1.200453 & -1.318256 \\
\hline $\mathrm{H}$ & 2.085540 & -0.252425 & -0.341321 \\
\hline $\mathrm{H}$ & 1.332040 & 0.052919 & 1.313806 \\
\hline $\mathrm{H}$ & -2.085880 & -0.252203 & -0.340925 \\
\hline $\mathrm{H}$ & -1.331537 & 0.053121 & 1.313835 \\
\hline \multicolumn{4}{|c|}{ cyclobutane } \\
\hline $\mathrm{C}$ & 0.000000 & 1.071275 & 0.147380 \\
\hline C & 1.071275 & 0.000000 & -0.147380 \\
\hline $\mathrm{H}$ & 1.344096 & 0.000000 & -1.207067 \\
\hline $\mathrm{C}$ & 0.000000 & -1.071275 & 0.147380 \\
\hline $\mathrm{H}$ & 1.986456 & 0.000000 & 0.451294 \\
\hline C & -1.071275 & 0.000000 & -0.147380 \\
\hline $\mathrm{H}$ & 0.000000 & -1.344096 & 1.207067 \\
\hline $\mathrm{H}$ & 0.000000 & -1.986456 & -0.451294 \\
\hline $\mathrm{H}$ & 0.000000 & 1.986456 & -0.451294 \\
\hline $\mathrm{H}$ & 0.000000 & 1.344096 & 1.207067 \\
\hline $\mathrm{H}$ & -1.344096 & 0.000000 & -1.207067 \\
\hline $\mathrm{H}$ & -1.986456 & 0.000000 & 0.451294 \\
\hline
\end{tabular}

cyclopentanylidene, singlet

$\begin{array}{llcc}\text { C } & 0.733029 & 0.957673 & -0.215594 \\ \mathrm{C} & 1.152803 & -0.485595 & 0.108304 \\ \mathrm{H} & 2.088932 & -0.844905 & -0.328642 \\ \mathrm{C} & -0.000012 & -1.415937 & -0.000009 \\ \mathrm{H} & 1.282702 & -0.585699 & 1.209233 \\ \mathrm{C} & -1.152814 & -0.485572 & -0.108298 \\ \mathrm{C} & -0.733007 & 0.957689 & 0.215591 \\ \mathrm{H} & 0.813395 & 1.132851 & -1.294984 \\ \mathrm{H} & 1.341746 & 1.712921 & 0.289066 \\ \mathrm{H} & -1.282757 & -0.585674 & -1.209221 \\ \mathrm{H} & -2.088933 & -0.844867 & 0.328682\end{array}$




$\begin{array}{lllr}\mathrm{H}-1.341708 & 1.712947 & -0.289073 \\ \mathrm{H}-0.813368 & 1.132876 & 1.294979\end{array}$

cyclopentanylidene, triplet

$\begin{array}{cccc}\text { C } & 0.729651 & -0.911253 & 0.238668 \\ \mathrm{C} & 1.250692 & 0.495625 & -0.128901 \\ \mathrm{H} & 2.060228 & 0.833713 & 0.526663 \\ \mathrm{C} & 0.000000 & 1.299919 & 0.000000 \\ \mathrm{H} & 1.641037 & 0.509163 & -1.156721 \\ \mathrm{C} & -1.250691 & 0.495625 & 0.128902 \\ \mathrm{C} & -0.729651 & -0.911253 & -0.238668 \\ \mathrm{H} & 0.758277 & -1.033384 & 1.326787 \\ \mathrm{H} & 1.324673 & -1.715484 & -0.204176 \\ \mathrm{H} & -1.641037 & 0.509163 & 1.156721 \\ \mathrm{H} & -2.060228 & 0.833714 & -0.526662 \\ \mathrm{H} & -1.324673 & -1.715483 & 0.204176 \\ \mathrm{H} & -0.758278 & -1.033384 & -1.326787\end{array}$

cyclopentyl+

$-0.000253$

C -0.000136

C -1.185180

C -0.735865

2.337538

0.000016

$1.245856-0.000010$

0.436557

$-0.101627$

$-0.999155 \quad 0.216515$

C 0.736104

$-0.998993$

$-0.216509$

C 1.185072

0.436825

0.101606

$\mathrm{H}-1.329580$

0.538277

$-1.213217$

$\mathrm{H}-2.099778$

0.847976

0.336349

$\mathrm{H}-0.830188$

$-1.172624$

1.293207

$\mathrm{H}-1.340237$

$-1.746323$

$-0.298795$

$\mathrm{H} \quad 0.830475$

$-1.172452$

$-1.293199$

H 1.340649

$-1.746012$

0.298814

H 2.099631

0.848457

$-0.336252$

H 1.329307

0.538622

1.213230

cyclopentane

C 0.000000

0.000000

1.298624

C 0.000000

1.234235

0.366021

$\mathrm{H}-0.998655$

1.683573

0.337772

C 0.342408

0.682353

$-1.021064$

H 0.691885

2.014051

0.697838

C -0.342408

$-0.682353$

$-1.021064$

H 1.426574

0.547662

$-1.118543$

H 0.016459

1.337508

$-1.834980$

C 0.000000

$-1.234235$

0.366021

$\mathrm{H}-0.877146$

0.004754

1.952302

H 0.877146

$-0.004754$

1.952302

$\mathrm{H}-1.426574$

$-0.547662$

$-1.118543$

$\mathrm{H}-0.016459$

$-1.337508$

$-1.834980$ 


$\begin{array}{llll}\mathrm{H} & -0.691885 & -2.014051 & 0.697838 \\ \mathrm{H} & 0.998655 & -1.683573 & 0.337772\end{array}$

phenylcarbene sets

\begin{tabular}{|c|c|c|}
\hline $\mathrm{H} \quad 0.837508$ & -2.164679 & 0.000000 \\
\hline C 0.285621 & -1.228504 & -0.000001 \\
\hline-1.042291 & 1.235405 & 0.000000 \\
\hline 1.041499 & -0.032758 & 0.000001 \\
\hline-1.103321 & -1.196885 & 0.000001 \\
\hline-1.762928 & 0.035609 & 0.000000 \\
\hline 0.345744 & 1.201690 & 0.000000 \\
\hline 2.477524 & -0.172873 & -0.000001 \\
\hline-1.678123 & -2.119040 & 0.000000 \\
\hline-2.850327 & 0.063647 & 0.000000 \\
\hline H 0.919007 & 2.126999 & 0.000001 \\
\hline-1.571955 & 2.184413 & -0.000001 \\
\hline 2.892805 & 0.858554 & 0.000001 \\
\hline
\end{tabular}

phenylcarbene, triplet, G3
$\mathrm{H} \mathrm{2.144621}$
0.887634
0.000000
C 1.215002
0.326277
0.000000
C -1.160476
$-1.080725$
0.000000
C 0.000000
1.028101
0.000000
C 1.229524
$-1.039506$
0.000000
C 0.045919
$-1.756569$
0.000000
C -1.192213
0.285962
0.000000
C -0.023672
2.436238
0.000000
$\mathrm{H} 2.179744$
$-1.566025$
0.000000
$\mathrm{H} 0.064333$
$-2.841915$
$\mathrm{H}-2.143614$
0.810816
0.000000
$\mathrm{H}-2.092767$
$-1.638517$
0.000000
$\mathrm{H}-0.836828$
3. 149344
0.000000
0.000000

benzyl+
C -2.345471
0.000000
$-0.000002$
C -0.973350
0.000000
$-0.000001$
C 1.798984
0.000000
0.000003
C -0.258792
$-1.245203$
$-0.000002$
C -0.258791
1.245204
$-0.000001$
C 1.119284
1.232445
0.000000
C 1.119284
$-1.232444$
0.000000
$\mathrm{H}-0.814708$
$-2.180063$
$-0.000012$
$\mathrm{H}-0.814708$
2.180063
$-0.000012$
H 1.681769
2. 160671
0.000002 


\begin{tabular}{|c|c|c|c|}
\hline $\mathrm{H}$ & 1.681768 & -2.160671 & 0.000002 \\
\hline $\mathrm{H}$ & 2.886975 & 0.000000 & 0.000003 \\
\hline & -2.913993 & 0.927303 & 0.000018 \\
\hline & -2.913993 & -0.927304 & 0.000017 \\
\hline & nzylt, per & ts & \\
\hline & -2.371531 & -0.000005 & 0.000007 \\
\hline & -0.950219 & -0.000013 & -0.000015 \\
\hline C & 1.820751 & 0.000013 & 0.000006 \\
\hline & -0.267627 & -1.235424 & 0.000001 \\
\hline & -0.267650 & 1.235412 & -0.000004 \\
\hline C & 1.125081 & 1.207681 & -0.000002 \\
\hline C & 1.125103 & -1.207669 & 0.000001 \\
\hline $\mathrm{H}$ & -0.801766 & -2.180642 & -0.000024 \\
\hline $\mathrm{H}$ & -0.801803 & 2.180623 & 0.000011 \\
\hline & 1.660961 & 2.152512 & -0.000007 \\
\hline $\mathrm{H}$ & 1.661000 & -2.152490 & 0.000010 \\
\hline $\mathrm{H}$ & 2.906291 & 0.000023 & 0.000005 \\
\hline & -2.954050 & 0.000011 & 0.926873 \\
\hline & -2.954082 & -0.000006 & -0.926839 \\
\hline
\end{tabular}

\begin{tabular}{|c|c|c|c|}
\hline \multicolumn{4}{|c|}{ toluene } \\
\hline $\mathrm{C}$ & -0.031126 & 2.417647 & 0.000000 \\
\hline C & 0.008507 & 0.912745 & 0.000000 \\
\hline $\mathrm{C}$ & 0.006381 & -1.900630 & 0.000000 \\
\hline $\mathrm{C}$ & 0.007243 & 0.195996 & 1.201554 \\
\hline $\mathrm{C}$ & 0.007243 & 0.195996 & -1.201554 \\
\hline $\mathrm{C}$ & 0.007243 & -1.198309 & -1.204938 \\
\hline $\mathrm{C}$ & 0.007243 & -1.198309 & 1.204938 \\
\hline $\mathrm{H}$ & 0.011874 & 0.737000 & 2.146329 \\
\hline $\mathrm{H}$ & 0.011874 & 0.737000 & -2.146329 \\
\hline $\mathrm{H}$ & 0.011111 & -1.736688 & -2.149649 \\
\hline $\mathrm{H}$ & 0.011111 & -1.736688 & 2.149649 \\
\hline $\mathrm{H}$ & 0.009217 & -2.987618 & 0.000000 \\
\hline $\mathrm{H}$ & -1.062531 & 2.786079 & 0.000000 \\
\hline $\mathrm{H}$ & 0.465472 & 2.825044 & -0.884895 \\
\hline & 0.465472 & 2.825044 & 0.884895 \\
\hline
\end{tabular}

phenylmethylcarbene, singlet
$\mathrm{H} 0.078315$
$-2.328066$
$-0.117863$
C 0.443842
$-1.306178$
$-0.060527$
C 1.313315
1.343749
$-0.045184$
C -0.508403
$-0.267462$
$-0.160798$
C 1.791475
$-1.025884$
0.132581
C 2.229045
0.301033
0.122446
C -0.045591
1.069226
$-0.150679$
C -1.886630
$-0.684895$
$-0.317312$ 


$\begin{array}{cccc}\mathrm{H} & 2.509565 & -1.834254 & 0.243664 \\ \mathrm{H} & 3.288100 & 0.523854 & 0.229279 \\ \mathrm{H} & -0.748555 & 1.888654 & -0.274324 \\ \mathrm{H} & 1.665125 & 2.372167 & -0.072171 \\ \mathrm{C}-2.882500 & 0.252629 & 0.247428 \\ \mathrm{H}-3.106437 & -0.236366 & 1.212563 \\ \mathrm{H}-3.825599 & 0.232956 & -0.305722 \\ \mathrm{H}-2.587825 & 1.287748 & 0.476842\end{array}$

phenylmethylcarbene, triplet

\begin{tabular}{|c|c|c|c|}
\hline $\mathrm{H}$ & 0.201362 & -2.343588 & 0.000000 \\
\hline C & 0.519362 & -1.305000 & 0.000000 \\
\hline $\mathrm{C}$ & 1.303803 & 1.339747 & 0.000000 \\
\hline C & -0.459249 & -0.299427 & -0.000001 \\
\hline $\mathrm{C}$ & 1.846336 & -0.986280 & 0.000000 \\
\hline $\mathrm{C}$ & 2.253911 & 0.336843 & -0.000001 \\
\hline & -0.030108 & 1.037214 & 0.000002 \\
\hline $\mathrm{C}$ & -1.831231 & -0.624089 & 0.000002 \\
\hline $\mathrm{H}$ & 2.588720 & -1.779641 & 0.000000 \\
\hline $\mathrm{H}$ & 3.310986 & 0.583922 & -0.000002 \\
\hline $\mathrm{H}$ & -0.767984 & 1.834684 & 0.000003 \\
\hline $\mathrm{H}$ & 1.616858 & 2.380389 & 0.000001 \\
\hline & -3.062729 & 0.187470 & -0.000001 \\
\hline & -3.122564 & 0.830867 & 0.887562 \\
\hline & -3.945805 & -0.457020 & -0.000451 \\
\hline & -3.122139 & 0.831519 & -0.887120 \\
\hline
\end{tabular}

phenylmethylcarbenium+

$\begin{array}{llcc}\text { H } & 0.703168 & 1.931663 & -0.000008 \\ \text { C } & -0.007076 & 1.111185 & -0.000001 \\ \text { C } & -1.855772 & -1.033494 & 0.000001 \\ \text { C } & 0.446942 & -0.243719 & -0.000045 \\ \text { C }-1.362711 & 1.368911 & -0.000005 \\ \text { C }-2.280636 & 0.303189 & 0.000017 \\ \text { C }-0.503090 & -1.312955 & -0.000005 \\ \text { C } 1.793722 & -0.582479 & -0.000002 \\ \text { H } & -1.725272 & 2.392111 & 0.000020 \\ \text { H } & -3.345995 & 0.522150 & 0.000034 \\ \text { H } & -0.147323 & -2.341028 & -0.000021 \\ \text { H }-2.585328 & -1.837142 & 0.000020 \\ \text { H } 2.022523 & -1.650476 & 0.000026 \\ \text { C } 2.963712 & 0.304865 & 0.000003 \\ \text { H } & 3.586531 & 0.060207 & -0.871555 \\ \text { H } 3.585615 & 0.061296 & 0.872523 \\ \text { H } 2.735535 & 1.368201 & -0.000824 \\ & & \\ \text { ethylbenzene } & & \\ \text { H }-0.269174 & 2.146733 & -0.310393\end{array}$




\begin{tabular}{|c|c|c|c|}
\hline C & 0.260241 & 1.202338 & -0.191858 \\
\hline C & 1.624666 & -1.205740 & 0.095339 \\
\hline $\mathrm{C}$ & -0.439661 & -0.000004 & -0.343638 \\
\hline C & 1.624660 & 1.205744 & 0.095334 \\
\hline C & 2.310799 & 0.000005 & 0.241770 \\
\hline C & 0.260247 & -1.202342 & -0.191852 \\
\hline & -1.924484 & -0.000008 & -0.592127 \\
\hline $\mathrm{H}$ & 2.153178 & 2.149997 & 0.202737 \\
\hline $\mathrm{H}$ & 3.375123 & 0.000008 & 0.462882 \\
\hline $\mathrm{H}$ & -0.269163 & -2.146740 & -0.310383 \\
\hline $\mathrm{H}$ & 2.153189 & -2.149990 & 0.202747 \\
\hline $\mathrm{H}$ & -2.196239 & -0.879240 & -1.187555 \\
\hline $\mathrm{C}$ & -2.717846 & 0.000006 & 0.716318 \\
\hline $\mathrm{H}$ & -2.473401 & -0.883595 & 1.312197 \\
\hline $\mathrm{H}$ & -3.795605 & 0.000001 & 0.527453 \\
\hline $\mathrm{H}$ & -2.473405 & 0.883623 & 1.312175 \\
\hline $\mathrm{H}$ & -2.196241 & 0.879209 & -1.187575 \\
\hline \multicolumn{4}{|c|}{ diphenylcarbene, singlet } \\
\hline C & -1.213380 & -0.601528 & 0.055090 \\
\hline $\mathrm{C}$ & 0.000000 & -1.372115 & 0.000001 \\
\hline $\mathrm{C}$ & 1.213380 & -0.601527 & -0.055086 \\
\hline $\mathrm{C}$ & -3.681134 & 0.744872 & 0.133208 \\
\hline $\mathrm{C}$ & -1.339428 & 0.644919 & 0.720107 \\
\hline C & -2.369521 & -1.165857 & -0.536037 \\
\hline $\mathrm{C}$ & -3.573552 & -0.475149 & -0.544272 \\
\hline $\mathrm{C}$ & -2.571530 & 1.287566 & 0.785959 \\
\hline $\mathrm{H}$ & -0.473219 & 1.064955 & 1.225122 \\
\hline $\mathrm{H}$ & -2.272258 & -2.143151 & -1.001889 \\
\hline $\mathrm{H}$ & -4.445441 & -0.901826 & -1.033831 \\
\hline $\mathrm{H}$ & -2.668226 & 2.224808 & 1.328947 \\
\hline $\mathrm{H}$ & -4.636873 & 1.262604 & 0.168297 \\
\hline $\mathrm{C}$ & 3.681134 & 0.744873 & -0.133212 \\
\hline C & 2.369524 & -1.165857 & 0.536036 \\
\hline $\mathrm{C}$ & 1.339426 & 0.644921 & -0.720102 \\
\hline $\mathrm{C}$ & 2.571528 & 1.287568 & -0.785957 \\
\hline C & 3.573554 & -0.475149 & 0.544268 \\
\hline $\mathrm{H}$ & 2.272263 & -2.143151 & 1.001887 \\
\hline $\mathrm{H}$ & 0.473215 & 1.064957 & -1.225114 \\
\hline $\mathrm{H}$ & 2.668222 & 2.224811 & -1.328945 \\
\hline $\mathrm{H}$ & 4.445445 & -0.901827 & 1.033823 \\
\hline $\mathrm{H}$ & 4.636873 & 1.262605 & -0.168304 \\
\hline \multicolumn{4}{|c|}{ diphenylcarbene, triplet } \\
\hline $\mathrm{C}$ & 1.316974 & -0.448226 & -0.017727 \\
\hline $\mathrm{C}$ & 0.000014 & -0.974938 & 0.000162 \\
\hline $\mathrm{C}$ & -1.316970 & -0.448240 & 0.017879 \\
\hline & 3.909144 & 0.546219 & -0.089545 \\
\hline
\end{tabular}




\begin{tabular}{|c|c|c|c|}
\hline C & 2.385836 & -1.197384 & 0.480925 \\
\hline C & 1.586874 & 0.808740 & -0.570528 \\
\hline $\mathrm{C}$ & 2.864502 & 1.294051 & -0.594136 \\
\hline C & 3.658629 & -0.703068 & 0.443066 \\
\hline $\mathrm{H}$ & 2.186447 & -2.177458 & 0.904269 \\
\hline $\mathrm{H}$ & 0.770175 & 1.389143 & -0.989320 \\
\hline $\mathrm{H}$ & 3.058272 & 2.272800 & -1.024380 \\
\hline $\mathrm{H}$ & 4.477914 & -1.296976 & 0.838394 \\
\hline $\mathrm{H}$ & 4.922159 & 0.935902 & -0.115194 \\
\hline $\mathrm{C}$ & -3.909152 & 0.546176 & 0.089364 \\
\hline C & -1.586958 & 0.808697 & 0.570691 \\
\hline $\mathrm{C}$ & -2.385748 & -1.197388 & -0.480952 \\
\hline $\mathrm{C}$ & -3.658551 & -0.703085 & -0.443260 \\
\hline C & -2.864591 & 1.294002 & 0.594127 \\
\hline $\mathrm{H}$ & -0.770321 & 1.389094 & 0.989615 \\
\hline $\mathrm{H}$ & -2.186287 & -2.177441 & -0.904311 \\
\hline $\mathrm{H}$ & -4.477774 & -1.296986 & -0.838727 \\
\hline $\mathrm{H}$ & -3.058432 & 2.272734 & 1.024380 \\
\hline $\mathrm{H}$ & -4.922174 & 0.935851 & 0.114878 \\
\hline \multicolumn{4}{|c|}{ benzhydryl+ } \\
\hline $\mathrm{H}$ & 2.239570 & 2.294877 & -0.614661 \\
\hline $\mathrm{C}$ & 2.391327 & 1.259645 & -0.316593 \\
\hline $\mathrm{C}$ & 2.745745 & -1.409099 & 0.476245 \\
\hline $\mathrm{C}$ & 1.270431 & 0.467866 & 0.055312 \\
\hline $\mathrm{C}$ & 3.655179 & 0.695688 & -0.352871 \\
\hline $\mathrm{C}$ & 3.832392 & -0.636777 & 0.041580 \\
\hline $\mathrm{C}$ & 1.468885 & -0.873621 & 0.483246 \\
\hline $\mathrm{C}$ & 0.000000 & 1.079450 & 0.000000 \\
\hline $\mathrm{H}$ & 4.509337 & 1.289063 & -0.664655 \\
\hline $\mathrm{H}$ & 4.829812 & -1.069053 & 0.038582 \\
\hline $\mathrm{H}$ & 0.640038 & -1.438814 & 0.898054 \\
\hline $\mathrm{H}$ & 2.910730 & -2.423042 & 0.828420 \\
\hline $\mathrm{C}$ & -1.270431 & 0.467866 & -0.055312 \\
\hline $\mathrm{H}$ & 0.000000 & 2.172822 & 0.000000 \\
\hline $\mathrm{C}$ & -3.832392 & -0.636777 & -0.041580 \\
\hline C & -2.391327 & 1.259645 & 0.316594 \\
\hline $\mathrm{C}$ & -1.468885 & -0.873621 & -0.483246 \\
\hline $\mathrm{C}$ & -2.745745 & -1.409099 & -0.476245 \\
\hline $\mathrm{C}$ & -3.655179 & 0.695688 & 0.352871 \\
\hline $\mathrm{H}$ & -2.239570 & 2.294877 & 0.614661 \\
\hline $\mathrm{H}$ & -0.640038 & -1.438814 & -0.898054 \\
\hline $\mathrm{H}$ & -2.910730 & -2.423042 & -0.828419 \\
\hline $\mathrm{H}$ & -4.509337 & 1.289063 & 0.664654 \\
\hline $\mathrm{H}$ & -4.829812 & -1.069053 & -0.038582 \\
\hline \multicolumn{4}{|c|}{ diphenylmethane } \\
\hline & & 1.724489 & 1.490327 \\
\hline
\end{tabular}




$\begin{array}{llrr}\text { C } & 2.248130 & 0.909728 & 0.778338 \\ \mathrm{C} 2.567251 & -1.173851 & -1.038850 \\ \mathrm{C} & 1.249714 & -0.061869 & 0.669347 \\ \mathrm{C} & 3.397200 & 0.844639 & -0.012010 \\ \mathrm{C} & 3.560205 & -0.198543 & -0.921507 \\ \mathrm{C} & 1.421041 & -1.103410 & -0.251464 \\ \mathrm{H} & 4.163979 & 1.609599 & 0.084297 \\ \mathrm{H} & 4.453860 & -0.251882 & -1.538082 \\ \mathrm{H} & 0.641399 & -1.856138 & -0.355620 \\ \mathrm{H} & 2.686762 & -1.989440 & -1.748045 \\ \mathrm{C}-1.249718 & 0.059655 & 0.669503 \\ \mathrm{C}-3.560194 & 0.201586 & -0.920912 \\ \mathrm{C}-2.248265 & -0.912141 & 0.775476 \\ \mathrm{C}-1.420910 & 1.104082 & -0.248058 \\ \mathrm{C}-2.567111 & 1.177122 & -1.035222 \\ \mathrm{C}-3.397325 & -0.844445 & -0.014667 \\ \mathrm{H}-2.127210 & -1.729134 & 1.484920 \\ \mathrm{H} & -0.641167 & 1.857025 & -0.349876 \\ \mathrm{H}-2.686515 & 1.994929 & -1.741875 \\ \mathrm{H}-4.164204 & -1.609600 & 0.079259 \\ \mathrm{H}-4.453841 & 0.256958 & -1.537320 \\ \mathrm{C}-0.000015 & -0.002495 & 1.514664 \\ \mathrm{H} & 0.049632 & 0.873911 & 2.173537 \\ \mathrm{H}-0.049684 & -0.881065 & 2.170647\end{array}$

$\underline{\text { meta-substituted arylcarbene sets }}$

3-oxyphenylcarbene, singlet

\begin{tabular}{|c|c|c|c|}
\hline C & -2.384917 & -1.273229 & 0.000013 \\
\hline C & -1.243881 & -0.425546 & -0.000012 \\
\hline C & 1.116297 & 1.080125 & 0.000067 \\
\hline & -1.308041 & 1.002013 & -0.000036 \\
\hline$C$ & 0.030368 & -1.058344 & 0.000069 \\
\hline C & 1.287993 & -0.359303 & -0.000018 \\
\hline C & -0.123471 & 1.725541 & -0.000007 \\
\hline $\mathrm{H}$ & -2.280066 & 1.493243 & -0.000113 \\
\hline $\mathrm{H}$ & 0.040309 & -2.147556 & 0.000097 \\
\hline 0 & 2.422591 & -0.924394 & -0.000061 \\
\hline $\mathrm{H}$ & -0.150281 & 2.817199 & 0.000018 \\
\hline 1 & 2.03386 & 1.670285 & 0.000097 \\
\hline & -3 & -0.585550 & -0.00006 \\
\hline
\end{tabular}

3-oxyphenylcarbene-, triplet, G3
C 2.428054
1.167871
0.000000
C 1.197568
0.392322
0.000000
C -1.150379
$-1.077172$
0.000000
C $1.274628-1.006829$
0.000000
C 0.000000
1.055341
0.000000 


\begin{tabular}{|c|c|c|c|}
\hline C & -1.276026 & 0.362742 & 0.000000 \\
\hline $\mathrm{C}$ & 0.071484 & -1.685612 & 0.000000 \\
\hline $\mathrm{H}$ & 2.230658 & -1.523081 & 0.000000 \\
\hline & -0.036869 & 2.143180 & 0.000000 \\
\hline & -2.368814 & 0.964666 & 0.000000 \\
\hline $\mathrm{H}$ & 0.096178 & -2.779775 & 0.000000 \\
\hline & -2.075125 & -1.651684 & 0.000000 \\
\hline $\mathrm{H}$ & 3.463693 & 0.842042 & 0.000000 \\
\hline & -oxybenzyl, & er ptsons & \\
\hline & 1.200101 & 0.307367 & 0.000000 \\
\hline & -1.226553 & -1.009915 & 0.000000 \\
\hline $\mathrm{C}$ & 1.186226 & -1.147603 & 0.000000 \\
\hline $\mathrm{C}$ & 0.000000 & 1.071938 & 0.000000 \\
\hline C & -1.309558 & 0.450499 & 0.000000 \\
\hline $\mathrm{C}$ & -0.036763 & -1.766919 & 0.000000 \\
\hline $\mathrm{H}$ & 2.125674 & -1.693752 & 0.000000 \\
\hline $\mathrm{H}$ & 0.056238 & 2.159294 & 0.000000 \\
\hline $\mathrm{O}$ & -2.390040 & 1.065109 & 0.000000 \\
\hline $\mathrm{H}$ & -0.102387 & -2.852815 & 0.000000 \\
\hline $\mathrm{H}$ & -2.185238 & -1.528071 & 0.000000 \\
\hline $\mathrm{C}$ & 2.416814 & 0.930669 & 0.00 \\
\hline $\mathrm{H}$ & 3.344692 & 0.363285 & 0.000000 \\
\hline $\mathrm{H}$ & 2.499743 & 2.014970 & 0.000000 \\
\hline \multicolumn{4}{|c|}{ 3-oxyphenyltoluene- } \\
\hline C & -1.160256 & -0.199990 & -0.022132 \\
\hline C & 1.373577 & 0.976718 & 0.012635 \\
\hline $\mathrm{C}$ & -1.050339 & 1.198516 & -0.009420 \\
\hline C & -0.015477 & -0.991509 & -0.010272 \\
\hline $\mathrm{C}$ & 1.327865 & -0.465499 & 0.000447 \\
\hline $\mathrm{C}$ & 0.230006 & 1.764925 & 0.002604 \\
\hline $\mathrm{H}$ & -1.941974 & 1.825512 & -0.025700 \\
\hline $\mathrm{H}$ & -0.108247 & -2.079590 & -0.024089 \\
\hline $\mathrm{O}$ & 2.367453 & -1.201162 & 0.001296 \\
\hline $\mathrm{H}$ & 0.332837 & 2.853315 & 0.004951 \\
\hline $\mathrm{H}$ & 2.362181 & 1.436778 & 0.017663 \\
\hline C & -2.525421 & -0.845686 & 0.016227 \\
\hline $\mathrm{H}$ & -3.264914 & -0.244046 & -0.525980 \\
\hline $\mathrm{H}$ & -2.896316 & -0.966225 & 1.042605 \\
\hline $\mathrm{H}$ & -2.502922 & -1.841299 & -0.440364 \\
\hline \multicolumn{4}{|c|}{ 3-aminophenylcarbene, singlet } \\
\hline C & 2.385868 & -1.372424 & 0.007665 \\
\hline C & 1.276844 & -0.452296 & -0.000192 \\
\hline C & -1.013936 & 1.154208 & -0.007546 \\
\hline $\mathrm{C}$ & 1.392879 & 0.959844 & 0.003509 \\
\hline & -0.012841 & -1.029854 & -0.009436 \\
\hline
\end{tabular}




$\begin{array}{llll}\mathrm{C} & -1.166478 & -0.243162 & -0.001228 \\ \mathrm{C} & 0.250025 & 1.748312 & 0.005715 \\ \mathrm{H} & 2.382258 & 1.411526 & 0.011817 \\ \mathrm{H} & -0.068990 & -2.116725 & -0.012480 \\ \mathrm{~N} & -2.438035 & -0.816211 & -0.082655 \\ \mathrm{H} & 0.325198 & 2.832764 & 0.007583 \\ \mathrm{H} & -1.903275 & 1.783117 & -0.015246 \\ \mathrm{H} & -2.476849 & -1.774079 & 0.248530 \\ \mathrm{H} & -3.173682 & -0.260960 & 0.340398 \\ \mathrm{H} & 3.307436 & -0.749932 & 0.007073\end{array}$

3-aminophenylcarbene, triplet

$\begin{array}{llcl}\mathrm{C} & 2.406531 & -1.263627 & 0.004561 \\ \mathrm{C} & 1.250112 & -0.448720 & 0.000121 \\ \mathrm{C} & -1.016974 & 1.141723 & -0.005896 \\ \mathrm{C} & 1.370215 & 0.945861 & 0.006418 \\ \mathrm{C} & -0.021852 & -1.024659 & -0.007060 \\ \mathrm{C} & -1.152154 & -0.243390 & -0.008196 \\ \mathrm{C} & 0.236537 & 1.710357 & 0.004121 \\ \mathrm{H} & 2.353142 & 1.406376 & 0.014635 \\ \mathrm{H} & -0.111136 & -2.108438 & -0.014166 \\ \mathrm{~N} & -2.418779 & -0.817989 & -0.078443 \\ \mathrm{H} & 0.321931 & 2.793991 & 0.008871 \\ \mathrm{H} & -1.905254 & 1.768275 & -0.016284 \\ \mathrm{H} & -2.464057 & -1.771337 & 0.261802 \\ \mathrm{H} & -3.157626 & -0.250385 & 0.319309 \\ \mathrm{H} & 3.459958 & -1.017826 & 0.010512\end{array}$

3-aminobenzyl+

\begin{tabular}{|c|c|c|c|}
\hline C & 1.232685 & -0.378039 & 0.001082 \\
\hline $\mathrm{C}$ & -1.080592 & 1.130465 & -0.002095 \\
\hline $\mathrm{C}$ & 1.326584 & 1.056876 & 0.001418 \\
\hline C & -0.031584 & -1.039679 & 0.001591 \\
\hline C & -1.211916 & -0.287133 & 0.004383 \\
\hline $\mathrm{C}$ & 0.157872 & 1.786877 & 0.001774 \\
\hline $\mathrm{H}$ & 2.302874 & 1.534271 & 0.002296 \\
\hline $\mathrm{H}$ & -0.064727 & -2.127347 & 0.000296 \\
\hline $\mathrm{N}$ & -2.438792 & -0.854725 & -0.031015 \\
\hline $\mathrm{H}$ & 0.180230 & 2.872233 & 0.000348 \\
\hline $\mathrm{H}$ & -1.989030 & 1.731789 & -0.008699 \\
\hline $\mathrm{H}$ & -2.549594 & -1.853531 & 0.079813 \\
\hline $\mathrm{H}$ & -3.271942 & -0.298093 & 0.101540 \\
\hline $\mathrm{C}$ & 2.392762 & -1.111101 & -0.000709 \\
\hline $\mathrm{H}$ & 3.366554 & -0.627121 & -0.001091 \\
\hline & 2.382309 & -2.198716 & -0.002064 \\
\hline & & & \\
\hline & & -0.239185 & -0.01903 \\
\hline
\end{tabular}




$\begin{array}{llcc}\text { C } & 1.266777 & 1.076643 & 0.016611 \\ \mathrm{C}-1.152133 & 1.157914 & -0.014755 \\ \mathrm{C}-0.016828 & -0.964064 & 0.000547 \\ \mathrm{C} & 1.227138 & -0.322696 & 0.002827 \\ \mathrm{C} & 0.081578 & 1.806234 & -0.003171 \\ \mathrm{H}-2.072606 & 1.737312 & -0.034600 \\ \mathrm{H}-0.051632 & -2.053683 & -0.001877 \\ \mathrm{~N} & 2.413859 & -1.071477 & 0.089217 \\ \mathrm{H} & 0.123465 & 2.892981 & -0.004192 \\ \mathrm{H} & 2.227017 & 1.589045 & 0.027350 \\ \mathrm{H} & 2.329618 & -1.998915 & -0.314542 \\ \mathrm{H} & 3.215318 & -0.594654 & -0.311451 \\ \mathrm{C}-2.534021 & -0.955446 & 0.009762 \\ \mathrm{H}-2.866132 & -1.132743 & 1.038263 \\ \mathrm{H} & -2.469788 & -1.926723 & -0.488877 \\ \mathrm{H} & -3.308842 & -0.368679 & -0.491306\end{array}$

3-hydroxyphenylcarbene, singlet

$\begin{array}{cccc}\text { C } & 2.411535 & 1.305261 & 0.000000 \\ \mathrm{C} & 1.273300 & 0.420716 & 0.000000 \\ \mathrm{C} & -1.067913 & -1.124338 & 0.000000 \\ \mathrm{C} & 1.342994 & -0.992898 & 0.000000 \\ \mathrm{C} & 0.000000 & 1.039119 & 0.000000 \\ \mathrm{C} & -1.159798 & 0.274210 & 0.000000 \\ \mathrm{C} & 0.177871 & -1.752460 & 0.000000 \\ \mathrm{H} & 2.318770 & -1.473446 & 0.000000 \\ \mathrm{H} & -0.025272 & 2.127708 & 0.000000 \\ \mathrm{H} & 0.225033 & -2.838224 & 0.000000 \\ \mathrm{H} & -1.989040 & -1.701432 & 0.000000 \\ \mathrm{H} & 3.312607 & 0.654376 & 0.000000 \\ \mathrm{O}-2.420317 & 0.804692 & 0.000000 \\ \mathrm{H} & -2.347506 & 1.775816 & 0.000000\end{array}$

3-hydroxyphenylcarbene, triplet
C 2.438631
1.170709
C 1.253544
0.403865
0.000000
C -1.082293
$-1.102660$
0.000000
C 1.310863
$-0.993184$
0.000000
C 0.000000
1.031481
0.000000
C -1.143308
0.284808
0.000000
C 0.146968
$-1.717688$
0.000000
$\mathrm{H} 2.274103$
$-1.493626$
0.000000
$\mathrm{H}-0.048287$
2. 118382
0.000000
$\mathrm{H} 0.193600$
$-2.803260$
0.000000
$\mathrm{H}-2.007600$
$-1.668320$
0.000000
$\mathrm{H} 3.482061$
0.886185
0.000000
O -2.392265
0.836368
0.000000
0.000000 


\begin{tabular}{|c|c|c|c|}
\hline $\mathrm{H}$ & -2.302188 & 1.805708 & 0.000000 \\
\hline \multicolumn{4}{|c|}{ 3-hydroxybenzyl+ } \\
\hline $\mathrm{C}$ & 1.234838 & 0.328655 & 0.000000 \\
\hline C & -1.151188 & -1.073789 & 0.000000 \\
\hline C & 1.252262 & -1.107153 & 0.000000 \\
\hline $\mathrm{C}$ & 0.000000 & 1.052783 & 0.000000 \\
\hline C & -1.190899 & 0.342228 & 0.000000 \\
\hline C & 0.052787 & -1.789642 & 0.000000 \\
\hline $\mathrm{H}$ & 2.204980 & -1.630887 & 0.000000 \\
\hline $\mathrm{H}$ & 0.014649 & 2.141128 & 0.000000 \\
\hline & 0.029412 & -2.874790 & 0.000000 \\
\hline & -2.105381 & -1.597230 & 0000 \\
\hline 0 & -2.421519 & 0.875983 & 0.000000 \\
\hline $\mathrm{H}$ & -2.392731 & 1.852 & 0000 \\
\hline C & 2.430276 & 1.003527 & 0.00 \\
\hline $\mathrm{H}$ & 3.378872 & 0.471767 & 0.000000 \\
\hline & 2.473909 & 2.09 & 0.0 \\
\hline \multicolumn{4}{|c|}{ 3-hydroxytoluene } \\
\hline C & -1.200553 & -0.219713 & -0.016739 \\
\hline C & 1.309735 & 1.0497 & 0.0 \\
\hline $\mathrm{C}$ & -1.109075 & 1.174092 & -0.007983 \\
\hline $\mathrm{C}$ & -0.021288 & -0.972043 & -0.009270 \\
\hline C & 1.222984 & -0.343156 & 0.0 \\
\hline $\mathrm{C}$ & 0.138518 & 1.799839 & 0.00 \\
\hline $\mathrm{H}$ & -2.017015 & 1.772923 & -0.017068 \\
\hline $\mathrm{H}$ & -0.077383 & -2.061301 & -0.019538 \\
\hline $\mathrm{H}$ & 0.199646 & 2.885364 & 0.003940 \\
\hline $\mathrm{H}$ & 2.289877 & 1.517026 & 0.013016 \\
\hline 0 & 2.407211 & -1.040589 & 0.002410 \\
\hline $\mathrm{H}$ & 2.201810 & -1.991941 & -0.004418 \\
\hline C & -2.538223 & -0.909822 & 0.012387 \\
\hline $\mathrm{H}$ & -3.318454 & -0.269066 & -0.406741 \\
\hline $\mathrm{H}$ & -2.8 & $-1 \cdot 1$ & 36905 \\
\hline $\mathrm{H}$ & -2.517708 & -1.838799 & -0.564581 \\
\hline \multicolumn{3}{|c|}{ 3-sulfhyrylphenylcarbene, } & singlet \\
\hline $\mathrm{C}$ & -2.513589 & -1.698375 & -0.000149 \\
\hline C & -1.581072 & -0.596175 & -0.000047 \\
\hline C & 0.397533 & 1.382978 & 0.000284 \\
\hline $\mathrm{C}$ & -1.937056 & 0.773072 & 0.000218 \\
\hline C & -0.210462 & -0.943364 & 0.000107 \\
\hline C & 0.782372 & 0.033263 & -0.000085 \\
\hline C & -0.949806 & 1.750082 & -0.000341 \\
\hline $\mathrm{H}$ & -2.989895 & 1.047004 & -0.000067 \\
\hline & 0.024492 & -2.005393 & -0.000320 \\
\hline S & 2.515111 & -0.331286 & -0.000068 \\
\hline & -1.213258 & 2.804488 & -0.000080 \\
\hline
\end{tabular}




\begin{tabular}{|c|c|c|c|}
\hline $\mathrm{H}$ & 1.161788 & 2.158095 & 0.000242 \\
\hline $\mathrm{H}$ & 2.377311 & -1.664344 & 0.000824 \\
\hline & -3.529730 & -1.248157 & 0.000572 \\
\hline \multicolumn{4}{|c|}{ 3-sulfhydrylphenylcarbene, } \\
\hline & 2.551904 & -1.590163 & 0.009949 \\
\hline & 1.562007 & -0.589525 & 0.003886 \\
\hline & -0.405672 & 1.374880 & -0.020032 \\
\hline & 1.907005 & 0.769474 & -0.003723 \\
\hline C & 0.201009 & -0.931938 & 0.020697 \\
\hline & -0.763568 & 0.034422 & 0.008786 \\
\hline $\mathrm{C}$ & 0.928451 & 1.725448 & -0.006921 \\
\hline $\mathrm{H}$ & 2.954761 & 1.055395 & -0.004403 \\
\hline $\mathrm{H}$ & -0.076363 & -1.981165 & 0.059015 \\
\hline S & -2.468719 & -0.477501 & -0.055222 \\
\hline $\mathrm{H}$ & 1.203551 & 2.776387 & -0.016932 \\
\hline & -1.171708 & 2.143507 & -0.053175 \\
\hline $\mathrm{H}$ & -2.929841 & 0.433608 & 3269 \\
\hline $\mathrm{H}$ & 3.632287 & -1.543307 & 0.009920 \\
\hline \multicolumn{4}{|c|}{ 3-sulfhydrylbenzyl+ } \\
\hline $\mathrm{C}$ & -1.544362 & -0.518836 & -0.000074 \\
\hline C & 0.452682 & 1.390192 & 0.000019 \\
\hline C & -1.900470 & 0.870159 & -0.000113 \\
\hline $\mathrm{C}$ & -0.172874 & -0.925392 & 0.000293 \\
\hline C & 0.838561 & 0.027603 & 0.000097 \\
\hline C & -0.888798 & 1.806403 & 0.000105 \\
\hline $\mathrm{H}$ & -2.948046 & 1.160901 & -0.000282 \\
\hline $\mathrm{H}$ & 0.062336 & -1.988549 & 0.000857 \\
\hline$S$ & 2.506290 & -0.515510 & -0.000157 \\
\hline $\mathrm{H}$ & -1.114638 & 2.868366 & 0.000189 \\
\hline $\mathrm{H}$ & 1.225933 & 2.156877 & -0.000173 \\
\hline $\mathrm{H}$ & 3.056359 & 0.706276 & 0.000657 \\
\hline C & -2.533858 & -1.471210 & -0.000080 \\
\hline $\mathrm{H}$ & -2.301935 & -2.533992 & 0.000138 \\
\hline $\mathrm{H}$ & -3.585945 & -1.195244 & -0.000353 \\
\hline \multicolumn{4}{|c|}{ 3-sulfhydryltoluene } \\
\hline C & 1.524404 & -0.401083 & -0.015826 \\
\hline C & -0.617278 & 1.417328 & 0.028664 \\
\hline C & 1.760802 & 0.978207 & -0.005009 \\
\hline C & 0.203115 & -0.854923 & -0.025509 \\
\hline $\mathrm{C}$ & -0.867575 & 0.043107 & 0.013365 \\
\hline C & 0.698025 & 1.878523 & 0.010527 \\
\hline $\mathrm{H}$ & 2.784434 & 1.347531 & -0.011534 \\
\hline $\mathrm{H}$ & 0.006074 & -1.924975 & -0.066626 \\
\hline S & -2.559238 & -0.507867 & -0.054320 \\
\hline & 0.892470 & 2.948226 & 0.019029 \\
\hline
\end{tabular}




\begin{tabular}{|c|c|c|c|}
\hline & -1.446526 & 2.118718 & 0.069106 \\
\hline $\mathrm{H}$ & -2.411740 & -1.551460 & 0.774843 \\
\hline & 2.668304 & -1.379282 & 0.002773 \\
\hline & 2.379210 & -2.333079 & -0.446513 \\
\hline & 2.997967 & -1.580316 & 1.027559 \\
\hline & 3.527140 & -0.990030 & -0.550670 \\
\hline \multicolumn{4}{|c|}{ 3-methylphenylcarbene, singlet } \\
\hline & -2.400884 & 1.376453 & 0.000000 \\
\hline & -1.295814 & 0.449749 & 0.000000 \\
\hline & 0.985701 & -1.167203 & 0.000000 \\
\hline & -1.416263 & -0.962700 & 0.000000 \\
\hline & 0.000000 & 1.012966 & 0.000000 \\
\hline $\mathrm{C}$ & 1.150240 & 0.224007 & 0.000000 \\
\hline $\mathrm{C}$ & -0.282418 & -1.761011 & 0.000000 \\
\hline $\mathrm{H}$ & -2.409170 & -1.408359 & 0.000000 \\
\hline & 0.062145 & 2.099840 & 0.000000 \\
\hline & -0.367909 & -2.844708 & 0.000000 \\
\hline $\mathrm{H}$ & 1.869178 & -1.804143 & 0.000000 \\
\hline $\mathrm{H}$ & -3.326327 & 0.759647 & 0.000000 \\
\hline & 2.518300 & 0.851408 & 0.000000 \\
\hline $\mathrm{H}$ & 3.302651 & 0.090435 & 0.000000 \\
\hline $\mathrm{H}$ & 2.658129 & 1.482635 & 0.882303 \\
\hline $\mathrm{H}$ & 2.658129 & 1.482635 & -0.882303 \\
\hline \multicolumn{4}{|c|}{ 3-methylphenylcarbene, triplet } \\
\hline $\mathrm{C}$ & -2.431834 & 1.247100 & 0.000000 \\
\hline $\mathrm{C}$ & -1.277641 & 0.437129 & 0.000000 \\
\hline $\mathrm{C}$ & 0.999609 & -1.145049 & 0.000000 \\
\hline $\mathrm{C}$ & -1.382529 & -0.963775 & 0.000000 \\
\hline C & 0.000000 & 1.006711 & 0.000000 \\
\hline $\mathrm{C}$ & 1.132337 & 0.232471 & 0.000000 \\
\hline $\mathrm{C}$ & -0.254944 & -1.730505 & 0.000000 \\
\hline $\mathrm{H}$ & -2.365434 & -1.426499 & 0.000000 \\
\hline $\mathrm{H}$ & 0.085182 & 2.091230 & 0.000000 \\
\hline $\mathrm{H}$ & -0.340885 & -2.813771 & 0.000000 \\
\hline $\mathrm{H}$ & 1.887697 & -1.771517 & 0.000000 \\
\hline $\mathrm{H}$ & -3.486414 & 1.006622 & 0.000000 \\
\hline & 2.494433 & 0.877658 & 0.000000 \\
\hline $\mathrm{H}$ & 3.285485 & 0.124295 & 0.000000 \\
\hline $\mathrm{H}$ & 2.628889 & 1.509600 & 0.882414 \\
\hline $\mathrm{H}$ & 2.628889 & 1.509600 & -0.882414 \\
\hline \multicolumn{4}{|c|}{ 3-methylbenzyl+ } \\
\hline C & -1.265926 & 0.336312 & 0.000000 \\
\hline C & 1.078667 & -1.121252 & 0.000000 \\
\hline C & -1.327500 & -1.097733 & 0.000000 \\
\hline & 0.000000 & 1.009749 & 0.000000 \\
\hline
\end{tabular}




\begin{tabular}{|c|c|c|c|}
\hline C & 1.182303 & 0.287808 & 0.000000 \\
\hline C & -0.149289 & -1.809568 & 0.000000 \\
\hline $\mathrm{H}$ & -2.294430 & -1.595271 & 0.000000 \\
\hline & 0.015863 & 2.098972 & 0.000000 \\
\hline & -0.156764 & -2.895119 & 0.000000 \\
\hline $\mathrm{H}$ & 1.997718 & -1.705567 & 0.000000 \\
\hline & 2.519116 & 0.974211 & 0.000000 \\
\hline & 3.335405 & 0.250282 & 0.000000 \\
\hline & 2.624612 & 1.608101 & 0.884168 \\
\hline & 2.624612 & 1.608101 & -0.884168 \\
\hline & -2.426188 & 1.068366 & 0.000000 \\
\hline $\mathrm{H}$ & -3.401657 & 0.587173 & 0.000000 \\
\hline $\mathrm{H}$ & -2.412458 & 2.155976 & 0.000000 \\
\hline \multicolumn{4}{|c|}{ 3-methyltoluene } \\
\hline C & -1.222446 & 0.267502 & 0.000000 \\
\hline C & 1.208235 & -1.130730 & 0.000000 \\
\hline C & -1.208235 & -1.130730 & 0.000000 \\
\hline $\mathrm{C}$ & 0.000000 & 0.945892 & 0.000000 \\
\hline $\mathrm{C}$ & 1.222446 & 0.267502 & 0.000000 \\
\hline C & 0.000000 & -1.825604 & 0.000000 \\
\hline $\mathrm{H}$ & -2.148865 & -1.678160 & 0.000000 \\
\hline $\mathrm{H}$ & 0.000000 & 2.036775 & 0.000000 \\
\hline $\mathrm{H}$ & 0.000000 & -2.913123 & 0.000000 \\
\hline $\mathrm{H}$ & 2.148865 & -1.678159 & 0.000000 \\
\hline C & 2.517459 & 1.036204 & 0.000000 \\
\hline $\mathrm{H}$ & 3.376071 & 0.359667 & 0.000000 \\
\hline $\mathrm{H}$ & 2.596312 & 1.678974 & 0.882484 \\
\hline $\mathrm{H}$ & 2.596312 & 1.678974 & -0.882484 \\
\hline $\mathrm{C}$ & -2.517459 & 1.036204 & 0.000000 \\
\hline $\mathrm{H}$ & -3.376071 & 0.359666 & 0.000000 \\
\hline $\mathrm{H}$ & -2.596311 & 1.678974 & -0.882484 \\
\hline $\mathrm{H}$ & -2.596311 & 1.678974 & 0.882484 \\
\hline \multicolumn{4}{|c|}{ 3-fluorophenylcarbene, } \\
\hline C & 2.417000 & 1.297067 & 0.000000 \\
\hline C & 1.264792 & 0.424361 & 0.000000 \\
\hline $\mathrm{C}$ & -1.091297 & -1.108500 & 0.000000 \\
\hline C & 1.319175 & -0.991094 & 0.000000 \\
\hline C & 0.000000 & 1.057330 & 0.000000 \\
\hline $\mathrm{C}$ & -1.145316 & 0.283047 & 0.000000 \\
\hline C & 0.152328 & -1.746455 & 0.000000 \\
\hline $\mathrm{H}$ & 2.291029 & -1.479444 & 0.000000 \\
\hline $\mathrm{H}$ & -0.047547 & 2.141796 & 0.000000 \\
\hline $\mathrm{H}$ & 0.194395 & -2.832055 & 0.000000 \\
\hline $\mathrm{H}$ & -2.019518 & -1.672824 & 0.000000 \\
\hline $\mathrm{H}$ & 3.308967 & 0.634301 & 0.000000 \\
\hline & -2.358602 & 0.879299 & 0.000000 \\
\hline
\end{tabular}


3-fluorophenylcarbene, triplet

$\begin{array}{cccc}\text { C } & 2.437960 & 1.165770 & 0.000000 \\ \text { C } & 1.247477 & 0.410169 & 0.000000 \\ \text { C } & -1.104310 & -1.086225 & 0.000000 \\ \text { C } & 1.287708 & -0.992367 & 0.000000 \\ \text { C } & 0.000000 & 1.047549 & 0.000000 \\ \text { C }-1.129019 & 0.290710 & 0.000000 \\ \text { C } & 0.126263 & -1.713322 & 0.000000 \\ \text { H } & 2.248069 & -1.498676 & 0.000000 \\ \text { H } & -0.072327 & 2.129540 & 0.000000 \\ \text { H } & 0.167956 & -2.798582 & 0.000000 \\ \text { H }-2.035459 & -1.641115 & 0.000000 \\ \text { H } & 3.479534 & 0.875227 & 0.000000 \\ \text { F }-2.331583 & 0.911100 & 0.000000\end{array}$

3-fluorobenzyl+

$\begin{array}{cccc}\text { C } & 1.228193 & 0.322240 & 0.000000 \\ \mathrm{C} & -1.181305 & -1.059697 & 0.000000 \\ \mathrm{C} & 1.224521 & -1.113103 & 0.000000 \\ \mathrm{C} & 0.000000 & 1.060545 & 0.000000 \\ \mathrm{C} & -1.176268 & 0.346806 & 0.000000 \\ \mathrm{C} & 0.018419 & -1.786688 & 0.000000 \\ \mathrm{H} & 2.170159 & -1.649813 & 0.000000 \\ \mathrm{H} & -0.008361 & 2.147550 & 0.000000 \\ \mathrm{H} & -0.012311 & -2.871650 & 0.000000 \\ \mathrm{H} & -2.142530 & -1.569547 & 0.000000 \\ \mathrm{~F} & -2.344160 & 0.981032 & 0.000000 \\ \mathrm{C} & 2.427090 & 0.992645 & 0.000000 \\ \mathrm{H} & 3.374454 & 0.458166 & 0.000000 \\ \mathrm{H} & 2.472135 & 2.079518 & 0.000000\end{array}$

3-fluorotoluene

$\begin{array}{cccc}\text { C } & 1.198915 & -0.200710 & -0.008355 \\ \text { C } & -1.350708 & 1.006417 & 0.004117 \\ \text { C } & 1.063998 & 1.191341 & -0.004263 \\ \text { C } & 0.042254 & -0.988360 & -0.005435 \\ \text { C } & -1.200102 & -0.373663 & 0.000480 \\ \text { C } & -0.197398 & 1.788209 & 0.001419 \\ \mathrm{H} & 1.954861 & 1.815066 & -0.008163 \\ \mathrm{H} & 0.096351 & -2.074019 & -0.010251 \\ \mathrm{H} & -0.284899 & 2.871490 & 0.002014 \\ \mathrm{H} & -2.344937 & 1.441370 & 0.006682 \\ \mathrm{~F} & -2.312332 & -1.152802 & 0.001053 \\ \mathrm{C} & 2.554140 & -0.855585 & 0.006276 \\ \mathrm{H} & 2.749971 & -1.332927 & 0.971707\end{array}$




\begin{tabular}{llcc} 
H & 3.345038 & -0.123148 & -0.172765 \\
H & 2.628006 & -1.628498 & -0.764135 \\
\multicolumn{3}{c}{ 3-chlorophenylcarbene, singlet } \\
C -1.594082 & -2.572448 & 0.000000 \\
C & -0.535514 & -1.587095 & 0.000000 \\
C & 1.364371 & 0.478593 & 0.000000 \\
C & 0.849029 & -1.879451 & 0.000000 \\
C & -0.946057 & -0.234424 & 0.000000 \\
C & 0.000000 & 0.780533 & 0.000000 \\
C & 1.785949 & -0.853792 & 0.000000 \\
H & 1.168738 & -2.919305 & 0.000000 \\
H & -2.011304 & -0.022583 & 0.000000 \\
H 2.849985 & -1.074259 & 0.000000 \\
H 2.090628 & 1.287078 & 0.000000 \\
H & -1.095302 & -3.565389 & 0.000000 \\
C1 -0.502642 & 2.441351 & 0.000000
\end{tabular}

3-chlorophenylcarbene, triplet

$\begin{array}{llcc}\text { C } & -1.479643 & -2.603282 & 0.000000 \\ \text { C } & -0.525666 & -1.567538 & 0.000000 \\ \text { C } & 1.353161 & 0.488658 & 0.000000 \\ \text { C } & 0.848995 & -1.849584 & 0.000000 \\ \text { C } & -0.933619 & -0.226083 & 0.000000 \\ \text { C } & 0.000000 & 0.765959 & 0.000000 \\ \text { C } & 1.760223 & -0.831282 & 0.000000 \\ \text { H } & 1.181071 & -2.883494 & 0.000000 \\ \text { H } & -1.991705 & 0.013873 & 0.000000 \\ \text { H } & 2.822232 & -1.058878 & 0.000000 \\ \text { H } & 2.074965 & 1.297909 & 0.000000 \\ \text { H } & -1.382187 & -3.680182 & 0.000000 \\ \text { C } 1-0.520299 & 2.426452 & 0.000000\end{array}$

3-chlorobenzyl+

$\begin{array}{llcc}\text { C } & -0.492528 & -1.539049 & 0.000000 \\ \text { C } & 1.373639 & 0.508808 & 0.000000 \\ \text { C } & 0.906378 & -1.852934 & 0.000000 \\ \text { C } & -0.939094 & -0.177451 & 0.000000 \\ \text { C } & 0.000000 & 0.834701 & 0.000000 \\ \text { C } & 1.822661 & -0.821047 & 0.000000 \\ \text { H } & 1.224164 & -2.892894 & 0.000000 \\ \text { H } & -2.003075 & 0.048680 & 0.000000 \\ \text { H } & 2.889047 & -1.024332 & 0.000000 \\ \text { H } & 2.098419 & 1.320687 & 0.000000 \\ \text { C } & -1.416539 & -2.555655 & 0.000000 \\ \text { H } & -1.110758 & -3.599541 & 0.000000 \\ \text { H } & -2.485417 & -2.353589 & 0.000000 \\ \text { C } 1-0.478793 & 2.477456 & 0.000000\end{array}$


3-chlorotoluene

$\begin{array}{llcc}\text { C } & 1.512552 & -0.390439 & -0.016595 \\ \mathrm{C} & -0.654840 & 1.408802 & 0.008226 \\ \mathrm{C} & 1.729838 & 0.991526 & -0.007720 \\ \mathrm{C} & 0.197380 & -0.864530 & -0.009849 \\ \mathrm{C} & -0.867304 & 0.032638 & 0.000325 \\ \mathrm{C} & 0.656602 & 1.880662 & 0.002473 \\ \mathrm{H} & 2.748323 & 1.374045 & -0.016370 \\ \mathrm{H} & -0.002090 & -1.933353 & -0.019523 \\ \mathrm{H} & 0.838797 & 2.952314 & 0.004473 \\ \mathrm{H} & -1.499140 & 2.091395 & 0.013045 \\ \mathrm{C} & 2.667488 & -1.355166 & 0.012132 \\ \mathrm{H} & 2.961584 & -1.585007 & 1.041503 \\ \mathrm{H} & 3.539922 & -0.937478 & -0.497165 \\ \mathrm{H} & 2.406833 & -2.297242 & -0.477130 \\ \mathrm{C} 1-2.496737 & -0.581508 & 0.001012\end{array}$

3-formylphenylcarbene, singlet

\begin{tabular}{|c|c|c|c|}
\hline C & -2.739696 & -1.421204 & 0.000000 \\
\hline & -1.640890 & -0.480704 & 0.000000 \\
\hline C & 0.621149 & 1.190747 & 0.000000 \\
\hline & -1.774099 & 0.928544 & 0.000000 \\
\hline & -0.338209 & -1.026661 & 0.000000 \\
\hline$C$ & 0.781213 & -0.201968 & 0.000000 \\
\hline$C$ & -0.653687 & 1.755065 & 0.000000 \\
\hline $\mathrm{H}$ & -2.773583 & 1.359926 & -0.000001 \\
\hline $\mathrm{H}$ & -0.247938 & -2.111890 & 0.000001 \\
\hline C & 2.144314 & -0.786032 & 0.000000 \\
\hline $\mathrm{H}$ & -0.766866 & 2.835909 & 0.000001 \\
\hline $\mathrm{H}$ & 1.512993 & 1.813383 & 0.000000 \\
\hline 0 & 3.170107 & -0.116896 & -0.000001 \\
\hline $\mathrm{H}$ & 2.185041 & -1.893608 & 0.000002 \\
\hline & -3.671077 & -0.815281 & 0.000000 \\
\hline
\end{tabular}

3-formylphenylcarbene, triplet

$\begin{array}{llcc}\text { C } & 2.739610 & -1.340512 & 0.000000 \\ \text { C } 1.617956 & -0.490695 & 0.000000 \\ \text { C }-0.610791 & 1.197597 & 0.000000 \\ \text { C } 1.756461 & 0.905770 & 0.000000 \\ \text { C } & 0.314109 & -1.012303 & 0.000000 \\ \text { C }-0.760452 & -0.178936 & 0.000000 \\ \text { C } & 0.659640 & 1.728925 & 0.000000 \\ \mathrm{H} & 2.754742 & 1.334840 & 0.000000 \\ \mathrm{H} & 0.175618 & -2.091845 & 0.000000 \\ \mathrm{C}-2.149481 & -0.767370 & 0.000001 \\ \mathrm{H} & 0.799196 & 2.805982 & 0.000000 \\ \mathrm{H}-1.498006 & 1.822754 & 0.000001\end{array}$




\begin{tabular}{|c|c|c|c|}
\hline 0 & -3.160481 & -0.137227 & -0.000002 \\
\hline $\mathrm{H}$ & -2.153043 & -1.883894 & 0.000004 \\
\hline & 3.803035 & -1.144877 & -0.000002 \\
\hline \multicolumn{4}{|c|}{ 3-formylbenzyl+ } \\
\hline $\mathrm{C}$ & 1.602913 & -0.404790 & 0.000001 \\
\hline & -0.685547 & 1.166517 & -0.000001 \\
\hline & 1.712833 & 1.026140 & -0.000002 \\
\hline $\mathrm{C}$ & 0.310723 & -1.028625 & 0.000000 \\
\hline $\mathrm{C}$ & -0.818291 & -0.239012 & 0.000004 \\
\hline $\mathrm{C}$ & 0.567007 & 1.797353 & 0.000003 \\
\hline $\mathrm{H}$ & 2.699368 & 1.484690 & 0.000000 \\
\hline $\mathrm{H}$ & 0.239040 & -2.116127 & 0.000003 \\
\hline $\mathrm{C}$ & -2.198528 & -0.830109 & 0.000001 \\
\hline $\mathrm{H}$ & 0.629148 & 2.881161 & -0.000001 \\
\hline $\mathrm{H}$ & -1.601583 & 1.756109 & -0.000001 \\
\hline $\mathrm{O}$ & -3.189131 & -0.120075 & -0.000003 \\
\hline $\mathrm{H}$ & -2.258534 & -1.932975 & 0.000004 \\
\hline $\mathrm{C}$ & 2.739708 & -1.174936 & -0.000002 \\
\hline $\mathrm{H}$ & 2.690260 & -2.261577 & 0.000000 \\
\hline $\mathrm{H}$ & 3.730456 & -0.725911 & -0.000002 \\
\hline \multicolumn{4}{|c|}{ 3-formyltoluene } \\
\hline C & -1.559498 & -0.314760 & -0.014864 \\
\hline $\mathrm{C}$ & 0.815831 & 1.210490 & 0.006231 \\
\hline $\mathrm{C}$ & -1.597595 & 1.084487 & -0.007671 \\
\hline C & -0.309485 & -0.939637 & -0.010533 \\
\hline $\mathrm{C}$ & 0.868130 & -0.187748 & -0.000782 \\
\hline C & -0.422804 & 1.841149 & 0.001822 \\
\hline $\mathrm{H}$ & -2.561787 & 1.590345 & -0.014992 \\
\hline $\mathrm{H}$ & -0.249191 & -2.028584 & -0.019626 \\
\hline C & 2.174316 & -0.877923 & -0.002315 \\
\hline $\mathrm{H}$ & -0.481205 & 2.926819 & 0.002860 \\
\hline $\mathrm{H}$ & 1.746175 & 1.772044 & 0.010843 \\
\hline $\mathrm{O}$ & 3.259180 & -0.305934 & 0.006341 \\
\hline $\mathrm{H}$ & 2.122162 & -1.987092 & -0.011917 \\
\hline C & -2.829626 & -1.121274 & 0.013349 \\
\hline $\mathrm{H}$ & -3.180090 & -1.265926 & 1.040621 \\
\hline & -2.678170 & -2.109522 & -0.428635 \\
\hline $\mathrm{H}$ & -3.626947 & -0.619313 & -0.541302 \\
\hline
\end{tabular}

3-trifluoromethylphenylcarbene, singlet
C -3.008655
$-1.742959$
0.000000
C -2.105765
$-0.611761$
0.000000
C -0.201843
1.450842
$-0.000005$
C -2.504715
0.744911
0.000004
C -0.724040
$-0.909614$
$-0.000006$
C 0.212113
0.113460
$-0.000009$ 


\begin{tabular}{|c|c|c|c|}
\hline C & -1.560603 & 1.766479 & 0.000001 \\
\hline $\mathrm{H}$ & -3.567423 & 0.979811 & 0.000008 \\
\hline $\mathrm{H}$ & -0.429859 & -1.954563 & -0.000012 \\
\hline $\mathrm{C}$ & 1.682302 & -0.167117 & -0.000001 \\
\hline $\mathrm{H}$ & -1.871389 & 2.807612 & 0.000002 \\
\hline $\mathrm{H}$ & 0.543052 & 2.243441 & -0.000011 \\
\hline $\mathrm{H}$ & -4.036745 & -1.321428 & 0.000008 \\
\hline $\mathrm{F}$ & 2.279574 & 0.374971 & 1.086074 \\
\hline $\mathrm{F}$ & 2.279648 & 0.375344 & -1.085849 \\
\hline $\mathrm{F}$ & 1.955178 & -1.485907 & -0.000215 \\
\hline
\end{tabular}

3-trifluoromethylphenylcarbene, triplet
C 3.031217
$-1.647715$
$-0.000001$
C 2.083254
$-0.607570$
$-0.000003$
C 0.200580
1.448707
$-0.000074$
C 2.477824
0.737382
0.000049
C 0.707834
$-0.892726$
$-0.000074$
C -0.200030
0.120615
$-0.000097$
C 1.545525
1.741942
0.000010
$\mathrm{H} 3.537031$
0.978708
H 0.380446
$-1.926385$
0.000102
C -1.668945
$-0.168841$
$-0.000132$
H 1.871958
2.777578
$-0.000001$
$\mathrm{H}-0.540731$
2.241391
0.000024
H 4.112497
$-1.647433$
$-0.000158$
F -2.275927
0.368078
0.000042
F -2.275399
0.366116
$-1.084415$
F -1.940313
$-1.491375$
1.085672
$-0.001116$

3-trifluoromethylbenzyl+
C 2.068617
$-0.539168$
0.000000
C 0.149388
1.464503
0.000001
C 2.469992
0.837311
0.000002
C 0.678163
$-0.890344$
$-0.000002$
C -0.259184
0.115743
$-0.000006$
C 1.505416
1.825327
$-0.000004$
$\mathrm{H} 3.529092$
1.085601
0.000002
$\mathrm{H} \quad 0.378878$
$-1.935548$
$-0.000004$
C -1.739694
$-0.180516$
0.000000
H 1.785481
2.874071
0.000001
$\mathrm{H}-0.611455$
2.243533
$-0.000003$
F -2.308495
0.360785
0.360717
$-1.088348$
F -2.308473
-1.501534
-1.528294
1.088392
F -1.969931
$-0.000039$
C 3.021937
$-1.528294$
0.000000
H 2.748093
$-1.293611$
$-0.000002$
H 4.084198
0.000002 
3-trifluoromethyltoluene

$\begin{array}{llcc}\text { C } & -2.034598 & -0.428253 & 0.012837 \\ \text { C }-0.027589 & 1.549494 & -0.032273 \\ \text { C }-2.363929 & 0.932179 & 0.022311 \\ \text { C }-0.684793 & -0.786367 & -0.020802 \\ \text { C } & 0.306120 & 0.195209 & -0.044776 \\ \text { C }-1.372210 & 1.911556 & -0.000063 \\ \text { H } & -3.411167 & 1.227414 & 0.050660 \\ \text { H } & -0.401725 & -1.836067 & -0.032652 \\ \text { C } & 1.742706 & -0.212652 & -0.001647 \\ \text { H } & -1.648322 & 2.962732 & 0.007925 \\ \text { H } & 0.751740 & 2.304915 & -0.053302 \\ \text { F } & 2.182536 & -0.356071 & 1.271523 \\ \text { F } & 2.547048 & 0.700236 & -0.593222 \\ \text { F } & 1.952706 & -1.396481 & -0.622833 \\ \text { C }-3.109156 & -1.481334 & -0.006772 \\ \text { H }-2.751407 & -2.417096 & 0.430184 \\ \text { H } & -3.431162 & -1.693642 & -1.031502 \\ \text { H } & -3.987878 & -1.156406 & 0.556584\end{array}$

3-cyanophenylcarbene, singlet

$\begin{array}{llcc}\mathrm{C} & -2.409345 & -1.722287 & 0.000000 \\ \mathrm{C} & -1.469155 & -0.618847 & 0.000000 \\ \mathrm{C} & 0.506513 & 1.381063 & 0.000001 \\ \mathrm{C} & -1.821360 & 0.751346 & -0.000002 \\ \mathrm{C} & -0.101840 & -0.966091 & 0.000002 \\ \mathrm{C} & 0.878188 & 0.026877 & 0.000002 \\ \mathrm{C} & -0.842383 & 1.738656 & 0.000000 \\ \mathrm{H} & -2.875174 & 1.023233 & -0.000004 \\ \mathrm{H} & 0.158775 & -2.021231 & 0.000003 \\ \mathrm{H} & -1.116980 & 2.789937 & -0.000001 \\ \mathrm{H} & 1.279128 & 2.146206 & 0.000003 \\ \mathrm{H} & -3.423287 & -1.268547 & -0.000001 \\ \mathrm{C} & 2.269597 & -0.325282 & 0.000003 \\ \mathrm{~N} & 3.416607 & -0.608887 & -0.000004\end{array}$

3-cyanophenylcarbene, triplet

$\begin{array}{llcc}\text { C } & 2.434043 & -1.620832 & 0.000000 \\ \text { C } & 1.446890 & -0.616953 & 0.000000 \\ \text { C } & -0.511903 & 1.372295 & 0.000001 \\ \text { C } & 1.789651 & 0.744410 & -0.000001 \\ \text { C } & 0.086122 & -0.951775 & 0.000001 \\ \text { C } & -0.856502 & 0.033255 & 0.000000 \\ \text { C } & 0.825742 & 1.713163 & 0.000000 \\ \text { H } & 2.839462 & 1.023495 & -0.000001 \\ \text { H } & -0.209410 & -1.996059 & 0.000002 \\ \text { H } & 1.113751 & 2.759872 & -0.000001 \\ \text { H } & -1.284107 & 2.133927 & 0.000001\end{array}$




\begin{tabular}{|c|c|c|c|}
\hline $\mathrm{H}$ & 3.514388 & -1.580255 & 0.000000 \\
\hline $\mathrm{C}$ & -2.268726 & -0.336603 & 0.000001 \\
\hline & -3.377999 & -0.623249 & -0.000002 \\
\hline \multicolumn{4}{|c|}{ 3-cyanobenzyl+ } \\
\hline $\mathrm{C}$ & 1.435527 & -0.546042 & -0.000001 \\
\hline C & -0.559382 & 1.386912 & -0.000004 \\
\hline C & 1.785506 & 0.843753 & 0.000004 \\
\hline $\mathrm{C}$ & 0.061862 & -0.951791 & -0.000007 \\
\hline C & -0.924133 & 0.018874 & -0.000008 \\
\hline C & 0.782644 & 1.793838 & 0.000002 \\
\hline $\mathrm{H}$ & 2.834258 & 1.132613 & 0.000009 \\
\hline $\mathrm{H}$ & -0.196208 & -2.008978 & -0.000010 \\
\hline $\mathrm{H}$ & 1.022226 & 2.852734 & 0.000006 \\
\hline $\mathrm{H}$ & -1.349258 & 2.136049 & -0.000005 \\
\hline $\mathrm{C}$ & -2.310028 & -0.346314 & -0.000008 \\
\hline $\mathrm{N}$ & -3.453135 & -0.646284 & 0.000014 \\
\hline C & 2.426210 & -1.499540 & 0.000003 \\
\hline $\mathrm{H}$ & 2.192904 & -2.562245 & 0.000002 \\
\hline $\mathrm{H}$ & 3.478775 & -1.224321 & 0.000008 \\
\hline \multicolumn{4}{|c|}{ 3-cyanotoluene } \\
\hline $\mathrm{C}$ & -1.411572 & -0.430740 & -0.015034 \\
\hline C & 0.725489 & 1.412370 & 0.007682 \\
\hline $\mathrm{C}$ & -1.647642 & 0.949616 & -0.007521 \\
\hline $\mathrm{C}$ & -0.090381 & -0.881459 & -0.009294 \\
\hline C & 0.971047 & 0.033291 & 0.000828 \\
\hline $\mathrm{C}$ & -0.592242 & 1.860770 & 0.002328 \\
\hline $\mathrm{H}$ & -2.672871 & 1.314941 & -0.015472 \\
\hline $\mathrm{H}$ & 0.125607 & -1.947703 & -0.017894 \\
\hline $\mathrm{H}$ & -0.797760 & 2.927967 & 0.003857 \\
\hline $\mathrm{H}$ & 1.557023 & 2.111435 & 0.012828 \\
\hline $\mathrm{C}$ & 2.321064 & -0.450015 & 0.000663 \\
\hline $\mathrm{N}$ & 3.433978 & -0.849600 & 0.001173 \\
\hline $\mathrm{C}$ & -2.556316 & -1.406900 & 0.011168 \\
\hline $\mathrm{H}$ & -3.399596 & -1.038337 & -0.578783 \\
\hline $\mathrm{H}$ & -2.910727 & -1.565634 & 1.034741 \\
\hline $\mathrm{H}$ & -2.256205 & -2.377066 & -0.392413 \\
\hline
\end{tabular}

3-nitrophenylcarbene, singlet
C 1.800205
$\begin{array}{lll}-0.586339 & -0.000008\end{array}$
C -0.157058
1.436808
0.000016
C 2.165715
C 0.429567
0.780160
0.000011
C -0.512796
$-0.923593$
$-0.000013$
C 1.196846
0.089990
0.000003
$\mathrm{H} 3.221989$
1.777631
0.000019
H 0.142419
1.041666
0.000016
$-1.969598$
$-0.000028$ 


$\begin{array}{clcr}\mathrm{H} & 1.481808 & 2.825983 & 0.000031 \\ \mathrm{H} & -0.933447 & 2.194507 & 0.000022 \\ \mathrm{~N} & -1.944383 & -0.258500 & 0.000000 \\ \mathrm{O} & -2.237798 & -1.462353 & 0.000059 \\ \mathrm{O} & -2.759715 & 0.676358 & -0.000062 \\ \mathrm{C} & 2.732322 & -1.698575 & -0.000031 \\ \mathrm{H} & 3.749200 & -1.251587 & -0.000005\end{array}$

3-nitrophenylcarbene, triplet

\begin{tabular}{|c|c|c|c|}
\hline C & -0.238123 & -1.858574 & 0.000000 \\
\hline $\mathrm{C}$ & 1.376049 & 0.426919 & 0.000000 \\
\hline C & 1.163128 & -1.951665 & 0.000000 \\
\hline $\mathrm{C}$ & -0.813127 & -0.582387 & 0.000000 \\
\hline $\mathrm{C}$ & 0.000000 & 0.508969 & 0.000000 \\
\hline $\mathrm{C}$ & 1.945615 & -0.829467 & 0.000000 \\
\hline $\mathrm{H}$ & 1.625873 & -2.934606 & 0.000000 \\
\hline $\mathrm{H}$ & -1.890002 & -0.460881 & 0.000000 \\
\hline $\mathrm{H}$ & 3.026833 & -0.927197 & 0.000000 \\
\hline $\mathrm{H}$ & 1.974203 & 1.329433 & 0.000000 \\
\hline $\mathrm{N}$ & -0.624412 & 1.847536 & 0.000000 \\
\hline $\mathrm{O}$ & -1.862196 & 1.907941 & 0.000000 \\
\hline 0 & 0.129951 & 2.831221 & 0.000000 \\
\hline $\mathrm{C}$ & -1.049240 & -3.011472 & 0.000000 \\
\hline $\mathrm{H}$ & -0.813876 & -4.066735 & 0.000000 \\
\hline
\end{tabular}

3-nitrobenzyl+

\begin{tabular}{|c|c|c|}
\hline-1.765038 & -0.513270 & -0.000001 \\
\hline 0.209743 & 1.447771 & 0.000001 \\
\hline-2.129298 & 0.873341 & 0.000001 \\
\hline-0.386362 & -0.904731 & -0.000001 \\
\hline 0.558290 & 0.089130 & -0.000002 \\
\hline-1.138759 & 1.836940 & 0.000002 \\
\hline-3.181154 & 1.150426 & 0.000001 \\
\hline-0.091877 & -1.951418 & -0.000001 \\
\hline-1.392056 & 2.892534 & 0.000004 \\
\hline 1.005952 & 2.188583 & 0.000003 \\
\hline 1.993069 & -0.279816 & 0.000000 \\
\hline 2.245738 & -1.489094 & 0.000005 \\
\hline 2.800294 & 0.656361 & -0.000005 \\
\hline-2.743105 & -1.479559 & -0.000001 \\
\hline-2.494148 & -2.538619 & -0.000003 \\
\hline-3.799280 & -1.218656 & -0.000001 \\
\hline
\end{tabular}

3-nitrotoluene

$\begin{array}{lll}\text { C }-1.740546 & -0.392191 & -0.004711 \\ \text { C } 0.369713 & 1.486848 & 0.001455 \\ \text { C }-1.999025 & 0.985235 & -0.003271\end{array}$




$\begin{array}{llcc}\mathrm{C} & -0.413610 & -0.826367 & -0.002675 \\ \mathrm{C} & 0.608245 & 0.117784 & 0.000128 \\ \mathrm{C} & -0.957599 & 1.911342 & -0.000384 \\ \mathrm{H} & -3.030009 & 1.334459 & -0.006165 \\ \mathrm{H} & -0.164991 & -1.882579 & -0.004685 \\ \mathrm{H} & -1.178397 & 2.975438 & -0.000677 \\ \mathrm{H} & 1.199223 & 2.184321 & 0.002565 \\ \mathrm{C} & -2.874428 & -1.381030 & 0.005115 \\ \mathrm{H} & -2.505865 & -2.403067 & -0.108660 \\ \mathrm{H} & -3.576665 & -1.179427 & -0.808911 \\ \mathrm{H} & -3.432767 & -1.325050 & 0.944680 \\ \mathrm{~N} & 1.999299 & -0.354280 & 0.000242 \\ \mathrm{O} & 2.193313 & -1.580920 & -0.003516 \\ \mathrm{O} & 2.898920 & 0.501688 & 0.004294\end{array}$

para-substituted arylcarbene sets

4-oxyphenylcarbene-, singlet

\begin{tabular}{|c|c|c|c|}
\hline C & -2.878696 & 0.143051 & 0.000001 \\
\hline C & -1.488533 & 0.026923 & -0.000003 \\
\hline C & 1.440496 & -0.009933 & 0.000000 \\
\hline C & -0.724837 & -1.199658 & -0.000002 \\
\hline C & -0.691844 & 1.230480 & -0.000002 \\
\hline $\mathrm{C}$ & 0.672357 & 1.228332 & 0.000002 \\
\hline C & 0.639592 & -1.229505 & 0.000002 \\
\hline $\mathrm{H}$ & -1.287886 & -2.136286 & -0.000003 \\
\hline $\mathrm{H}$ & -1.254356 & 2.164441 & 0.000003 \\
\hline $\mathrm{H}]$ & .247984 & 2.153128 & 0.000001 \\
\hline $\mathrm{H}$ & 1.192926 & -2.168096 & 0.000006 \\
\hline $\mathrm{O}$ & 2.695667 & -0.030507 & -0.000001 \\
\hline & -3.275 & -0.907268 & 0.00000 \\
\hline
\end{tabular}

4-oxyphenylcarbene-, triplet
C 2.881528
0.090647
$-0.000052$
C 1.459445
0.057573
0.000000
C -1.448886
$-0.025208$
$-0.000010$
C 0.731291
$-1.148794$
$-0.000012$
C 0.663959
1.220158
0.000008
C -0.687466
1.210811
0.000000
C -0.619837
$-1.216428$
$-0.000002$
$\mathrm{H} 1.295504$
$-2.085351$
$-0.000040$
$\mathrm{H} 1.177893$
2. 184296
0.000004
H -1.263967
2.134715
0.000014
$\mathrm{H}-1.142442$
$-2.171856$
0.000007
O -2.694350
$-0.059787$
0.000013 


\begin{tabular}{|c|c|c|c|}
\hline & 3.607614 & -0.716060 & 0.000322 \\
\hline \multicolumn{4}{|c|}{ 4-oxyphenylbenzyl (p-quinomethide) } \\
\hline & 0.000000 & 0.000000 & -1.398304 \\
\hline $\mathrm{C}$ & 0.000000 & 0.000000 & 1.481212 \\
\hline $\mathrm{C}$ & 0.000000 & 1.246070 & -0.644812 \\
\hline C & 0.000000 & -1.246070 & -0.644812 \\
\hline $\mathrm{C}$ & 0.000000 & -1.251517 & 0.706791 \\
\hline $\mathrm{C}$ & 0.000000 & 1.251517 & 0.706791 \\
\hline $\mathrm{H}$ & 0.000000 & 2.180007 & -1.204586 \\
\hline $\mathrm{H}$ & 0.000000 & -2.180007 & -1.204586 \\
\hline $\mathrm{H}$ & 0.000000 & -2.175540 & 1.278461 \\
\hline $\mathrm{H}$ & 0.000000 & 2.175540 & 1.278461 \\
\hline $\mathrm{O}$ & 0.000000 & 0.000000 & 2.720516 \\
\hline $\mathrm{C}$ & 0.000000 & 0.000000 & -2.752254 \\
\hline $\mathrm{H}$ & 0.000000 & 0.926014 & -3.319775 \\
\hline 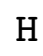 & 0.000000 & -0.926014 & -3.319775 \\
\hline \multicolumn{4}{|c|}{ 4-oxytoluene- } \\
\hline $\mathrm{C}$ & -1.342716 & 0.000002 & -0.028161 \\
\hline $\mathrm{C}$ & 1.583584 & 0.000001 & 0.008098 \\
\hline $\mathrm{C}$ & -0.604150 & -1.193027 & -0.014512 \\
\hline C & -0.604149 & 1.193027 & -0.014510 \\
\hline C & 0.785308 & 1.202220 & -0.008702 \\
\hline $\mathrm{C}$ & 0.785305 & -1.202219 & -0.008700 \\
\hline $\mathrm{H}$ & -1.141727 & -2.146580 & -0.027525 \\
\hline $\mathrm{H}$ & -1.141727 & 2.146579 & -0.027523 \\
\hline $\mathrm{H}$ & 1.327221 & 2.148755 & -0.010122 \\
\hline $\mathrm{H}$ & 1.327219 & -2.148754 & -0.010121 \\
\hline 0 & 2.857022 & -0.000003 & 0.022210 \\
\hline $\mathrm{C}$ & -2.846322 & -0.000002 & 0.029793 \\
\hline $\mathrm{H}$ & -3.263177 & 0.883799 & -0.469143 \\
\hline $\mathrm{H}$ & -3.241908 & -0.000362 & 1.056693 \\
\hline n & -3.263229 & -0.883426 & -0.469772 \\
\hline
\end{tabular}

4-aminophenylcarbene, singlet

$\begin{array}{llcc}\text { C } & 2.949926 & -0.142876 & 0.012079 \\ \mathrm{C} & 1.527130 & -0.021332 & 0.001117 \\ \mathrm{C} & -1.304572 & 0.006249 & 0.000317 \\ \mathrm{C} & 0.797313 & 1.198120 & -0.001576 \\ \mathrm{C} & 0.771890 & -1.222783 & -0.000976 \\ \mathrm{C} & -0.607304 & -1.218477 & -0.008216 \\ \mathrm{C} & -0.581677 & 1.217971 & -0.008155 \\ \mathrm{H} & 1.349740 & 2.136266 & -0.001333 \\ \mathrm{H} & 1.331164 & -2.154746 & 0.000609 \\ \mathrm{H} & -1.168551 & -2.150761 & -0.011726 \\ \mathrm{H} & -1.125328 & 2.160850 & -0.012543 \\ \mathrm{H} & 3.354328 & 0.894094 & 0.012575\end{array}$




\begin{tabular}{|c|c|c|c|}
\hline & -2.678945 & 0.022787 & -0.058982 \\
\hline & -3.161889 & -0.820111 & 0.225248 \\
\hline & -3.143087 & 0.873661 & 0.232497 \\
\hline \multicolumn{4}{|c|}{ 4-aminophenylcarbene, triplet } \\
\hline & -2.920718 & 0.085303 & 0.012287 \\
\hline $\mathrm{C}$ & -1.511425 & 0.050426 & 0.002067 \\
\hline C & 1.300381 & -0.017384 & -0.005410 \\
\hline C & -0.803477 & -1.160272 & -0.000450 \\
\hline C & -0.746285 & 1.225086 & -0.000058 \\
\hline C & 0.614474 & 1.193268 & -0.008073 \\
\hline C & 0.559061 & -1.193026 & -0.008459 \\
\hline $\mathrm{H}$ & -1.356697 & -2.096007 & 0.001503 \\
\hline $\mathrm{H}$ & -1.259399 & 2.182719 & 0.002093 \\
\hline $\mathrm{H}$ & 1.178020 & 2.123835 & -0.014397 \\
\hline $\mathrm{H}$ & 1.077185 & -2.149688 & -0.014905 \\
\hline $\mathrm{H}$ & -3.665017 & -0.699437 & 0.013140 \\
\hline & 2.687759 & -0.048944 & -0.073180 \\
\hline & 3.148759 & 0.777609 & 0.288179 \\
\hline $\mathrm{H}$ & 3.110766 & -0.896827 & 0.285222 \\
\hline \multicolumn{4}{|c|}{ 4-aminobenzyl+ } \\
\hline $\mathrm{C}$ & -2.816118 & 0.000000 & 0.000003 \\
\hline C & -1.455662 & 0.000000 & -0.000002 \\
\hline $\mathrm{C}$ & 1.358983 & 0.000000 & 0.000004 \\
\hline C & -0.714659 & 1.240348 & 0.000003 \\
\hline & -0.714659 & -1.240348 & 0.000004 \\
\hline $\mathrm{C}$ & 0.644554 & -1.244237 & -0.000011 \\
\hline C & 0.644554 & 1.244237 & -0.000011 \\
\hline $\mathrm{H}$ & -1.263124 & 2.179382 & 0.000002 \\
\hline $\mathrm{H}$ & -1.263124 & -2.179381 & 0.000002 \\
\hline $\mathrm{H}$ & 1.201242 & -2.177716 & -0.000004 \\
\hline $\mathrm{H}$ & 1.201242 & 2.177716 & -0.000004 \\
\hline $\mathrm{N}$ & 2.682463 & 0.000000 & 0.000005 \\
\hline $\mathrm{H}$ & 3.216265 & 0.864208 & 0.000012 \\
\hline $\mathrm{H}$ & 3.216265 & -0.864208 & 0.000012 \\
\hline & -3.383979 & 0.926341 & 0.000004 \\
\hline $\mathrm{H}$ & -3.383979 & -0.926341 & 0.000005 \\
\hline \multicolumn{4}{|c|}{ 4-aminotoluene } \\
\hline $\mathrm{C}$ & 1.397145 & -0.000005 & -0.017831 \\
\hline C & -1.436308 & 0.000000 & 0.005142 \\
\hline C & 0.672516 & -1.196072 & -0.009740 \\
\hline C & 0.672518 & 1.196069 & -0.009744 \\
\hline $\mathrm{C}$ & -0.719397 & 1.202536 & 0.007541 \\
\hline $\mathrm{C}$ & -0.719403 & -1.202535 & 0.007540 \\
\hline $\mathrm{H}$ & 1.205377 & -2.145706 & -0.017858 \\
\hline $\mathrm{H}$ & 1.205376 & 2.145704 & -0.017863 \\
\hline
\end{tabular}




\begin{tabular}{|c|c|c|c|}
\hline $\mathrm{H}$ & -1.258053 & 2.148809 & 0.012507 \\
\hline & -1.258063 & -2.148805 & 0.012509 \\
\hline & 2.901982 & 0.000001 & 0.006770 \\
\hline & -2.839615 & 0.000000 & 0.093113 \\
\hline & 3.285474 & -0.000017 & 1.032875 \\
\hline & 3.304152 & 0.884365 & -0.495500 \\
\hline & 3.304155 & -0.884337 & -0.495549 \\
\hline & -3.262715 & -0.829686 & -0.310506 \\
\hline & -3.262710 & 0.829705 & -0.310472 \\
\hline \multicolumn{3}{|c|}{ 4-hydroxyphenylcarbene, } & singlet \\
\hline & -2.931660 & -0.163943 & 0.000000 \\
\hline & -1.504888 & -0.027834 & 0.000001 \\
\hline & 1.302995 & 0.021809 & -0.000002 \\
\hline & -0.789275 & 1.199969 & 0.000000 \\
\hline C & -0.748516 & -1.225038 & -0.000001 \\
\hline C & 0.635230 & -1.210309 & 0.000001 \\
\hline C & 0.591364 & 1.231585 & 0.000002 \\
\hline $\mathrm{H}$ & -1.350808 & 2.132368 & 0.000003 \\
\hline $\mathrm{H}$ & -1.302260 & -2.160094 & 0.000000 \\
\hline $\mathrm{H}$ & 1.204732 & -2.138370 & -0.000003 \\
\hline $\mathrm{H}$ & 1.151395 & 2.162044 & 0.000000 \\
\hline $\mathrm{O}$ & 2.658647 & 0.119366 & -0.000003 \\
\hline $\mathrm{H}$ & 3.043153 & -0.776115 & 0.000025 \\
\hline $\mathrm{H}$ & -3.346884 & 0.867795 & 0.000000 \\
\hline \multicolumn{3}{|c|}{ 4-hydroxyphenylcarbene, } & triplet \\
\hline C & 0.032814 & -2.901773 & 0.000000 \\
\hline $\mathrm{C}$ & 0.023682 & -1.490991 & 0.000000 \\
\hline C & 0.000000 & 1.299493 & 0.000000 \\
\hline $\mathrm{C}$ & -1.180730 & -0.765920 & 0.000000 \\
\hline $\mathrm{C}$ & 1.210687 & -0.752734 & 0.000000 \\
\hline $\mathrm{C}$ & 1.200305 & 0.614839 & 0.000000 \\
\hline $\mathrm{C}$ & -1.193699 & 0.594502 & 0.000000 \\
\hline $\mathrm{H}$ & -2.122963 & -1.307324 & 0.000000 \\
\hline $\mathrm{H}$ & 2.158955 & -1.282162 & 0.000000 \\
\hline $\mathrm{H}$ & 2.139913 & 1.164321 & 0.000000 \\
\hline $\mathrm{H}$ & -2.125793 & 1.150626 & 0.000000 \\
\hline $\mathrm{O}$ & -0.082787 & 2.661697 & 0.000000 \\
\hline $\mathrm{H}$ & 0.818724 & 3.028411 & 0.000000 \\
\hline $\mathrm{H}$ & -0.764893 & -3.631949 & 0.000000 \\
\hline \multicolumn{4}{|c|}{ 4-hydroxybenzyl+ } \\
\hline $\mathrm{C}$ & -0.006879 & -1.432223 & 0.000000 \\
\hline C & 0.000000 & 1.354761 & 0.000000 \\
\hline $\mathrm{C}$ & -1.247931 & -0.697931 & 0.000000 \\
\hline & 1.237143 & -0.704526 & 0.000000 \\
\hline & 1.241948 & 0.660947 & 0.000000 \\
\hline
\end{tabular}




\begin{tabular}{|c|c|c|c|}
\hline I & -1.243959 & 0.666533 & 0.000000 \\
\hline & -2.187117 & -1.246011 & 0.000000 \\
\hline 1 & 2.173740 & -1.257377 & 0.000000 \\
\hline & 2.176161 & 1.217467 & 0.000000 \\
\hline & -2.157074 & 1.253461 & 0.000000 \\
\hline & -0.080638 & 2.669483 & 0.000000 \\
\hline & 0.800275 & 3.100523 & 0.000000 \\
\hline & -0.005440 & -2.796628 & 0.000000 \\
\hline & 0.921619 & -3.364210 & 0.000000 \\
\hline & -0.931789 & -3.365311 & 0.000000 \\
\hline
\end{tabular}

\begin{tabular}{lcc}
\multicolumn{4}{l}{-hydroxytoluene } \\
C 1.377992 & 0.007414 & -0.016269 \\
C -1.435642 & -0.015142 & 0.004033 \\
C 0.663124 & -1.197125 & -0.009181 \\
C 0.649021 & 1.199031 & -0.009119 \\
C -0.745754 & 1.196873 & -0.002623 \\
C -0.727452 & -1.217772 & -0.002310 \\
H 1.205448 & -2.141055 & -0.017449 \\
H 1.175723 & 2.151601 & -0.017203 \\
H -1.292525 & 2.139055 & -0.003402 \\
H -1.280936 & -2.152390 & -0.002466 \\
O -2.807410 & -0.096766 & 0.008981 \\
H -3.167660 & 0.807236 & 0.011619 \\
C 2.882841 & 0.014013 & 0.015831 \\
H 3.280916 & 0.945600 & -0.395503 \\
H 3.292849 & -0.814445 & -0.568626 \\
H 3.260679 & -0.085231 & 1.039008 \\
& & \\
4-Sulfhydrylphenylcarbene & singlet \\
C -3.378286 & 0.136848 & 0.000000 \\
C -1.945433 & 0.018960 & 0.000000 \\
C 0.876623 & 0.003153 & 0.000000 \\
C -1.218395 & -1.198714 & 0.000000 \\
C -1.203147 & 1.223459 & 0.000000 \\
C 0.181895 & 1.223121 & 0.000000 \\
C 0.164733 & -1.210154 & 0.000000 \\
H -1.767137 & -2.138810 & 0.000000 \\
H -1.765352 & 2.153729 & 0.000000 \\
H 0.729883 & 2.162467 & 0.000000 \\
H 0.707680 & -2.152686 & 0.000000 \\
S 2.634161 & -0.095648 & 0.000000 \\
H 2.859866 & 1.225781 & 0.000000 \\
H -3.779455 & -0.900143 & 0.000000
\end{tabular}




\begin{tabular}{|c|c|c|c|}
\hline \multicolumn{4}{|c|}{ 4-sulfhydrylphenylcarbene, triplet } \\
\hline $\mathrm{C}$ & -3.335143 & 0.071648 & -0.001699 \\
\hline $\mathrm{C}$ & -1.930682 & 0.044856 & -0.001687 \\
\hline $\mathrm{C}$ & 0.866172 & -0.002970 & 0.010509 \\
\hline $\mathrm{C}$ & -1.222494 & -1.167578 & 0.009859 \\
\hline $\mathrm{C}$ & -1.180583 & 1.232292 & -0.007888 \\
\hline $\mathrm{C}$ & 0.181372 & 1.203884 & -0.017853 \\
\hline $\mathrm{C}$ & 0.142532 & -1.183858 & 0.021914 \\
\hline $\mathrm{H}$ & -1.772299 & -2.104614 & 0.021218 \\
\hline $\mathrm{H}$ & -1.703642 & 2.183959 & -0.020471 \\
\hline $\mathrm{H}$ & 0.734651 & 2.138939 & -0.045094 \\
\hline $\mathrm{H}$ & 0.667441 & -2.134712 & 0.055675 \\
\hline S & 2.634717 & -0.099877 & -0.044589 \\
\hline $\mathrm{H}$ & 2.869994 & 1.038353 & 0.623012 \\
\hline $\mathrm{H}$ & -4.078658 & -0.713537 & 0.000167 \\
\hline & -sulfhydry & enzyl+ & \\
\hline $\mathrm{C}$ & 1.868043 & -0.001507 & 0.000000 \\
\hline $\mathrm{C}$ & -0.942937 & 0.008468 & 0.000000 \\
\hline $\mathrm{C}$ & 1.132690 & -1.238681 & 0.000000 \\
\hline $\mathrm{C}$ & 1.139469 & 1.239472 & 0.000000 \\
\hline C & -0.227270 & 1.241375 & 0.000000 \\
\hline $\mathrm{C}$ & -0.233169 & -1.229393 & 0.000000 \\
\hline $\mathrm{H}$ & 1.676862 & -2.180393 & 0.000000 \\
\hline $\mathrm{H}$ & 1.689597 & 2.177908 & 0.000000 \\
\hline $\mathrm{H}$ & -0.769976 & 2.182737 & 0.000000 \\
\hline $\mathrm{H}$ & -0.788308 & -2.163981 & 0.000000 \\
\hline S & -2.646867 & -0.084351 & 0.000000 \\
\hline $\mathrm{H}$ & -2.887807 & 1.235992 & 0.000000 \\
\hline C & 3.234091 & -0.002503 & 0.000000 \\
\hline $\mathrm{H}$ & 3.802839 & 0.923880 & 0.000000 \\
\hline $\mathrm{H}$ & 3.801158 & -0.929906 & 0.000000 \\
\hline & -sulfhydry] & oluene & \\
\hline $\mathrm{C}$ & -1.822906 & 0.000676 & -0.015029 \\
\hline $\mathrm{C}$ & 0.998769 & -0.001805 & -0.010683 \\
\hline C & -1.100453 & 1.199053 & -0.002795 \\
\hline $\mathrm{C}$ & -1.103833 & -1.198203 & -0.020416 \\
\hline $\mathrm{C}$ & 0.289654 & -1.205394 & -0.027401 \\
\hline C & 0.291797 & 1.204136 & 0.019940 \\
\hline $\mathrm{H}$ & -1.634832 & 2.147574 & 0.004147 \\
\hline $\mathrm{H}$ & -1.640082 & -2.145396 & -0.038076 \\
\hline $\mathrm{H}$ & 0.829504 & -2.148183 & -0.066772 \\
\hline $\mathrm{H}$ & 0.829586 & 2.148814 & 0.053551 \\
\hline $\mathrm{S}$ & 2.776672 & -0.059822 & 0.063373 \\
\hline $\mathrm{H}$ & 2.998550 & 0.923933 & -0.820687 \\
\hline $\mathrm{C}$ & -3.327190 & 0.004094 & 0.017709 \\
\hline $\mathrm{H}$ & -3.700121 & 0.029446 & 1.047169 \\
\hline
\end{tabular}




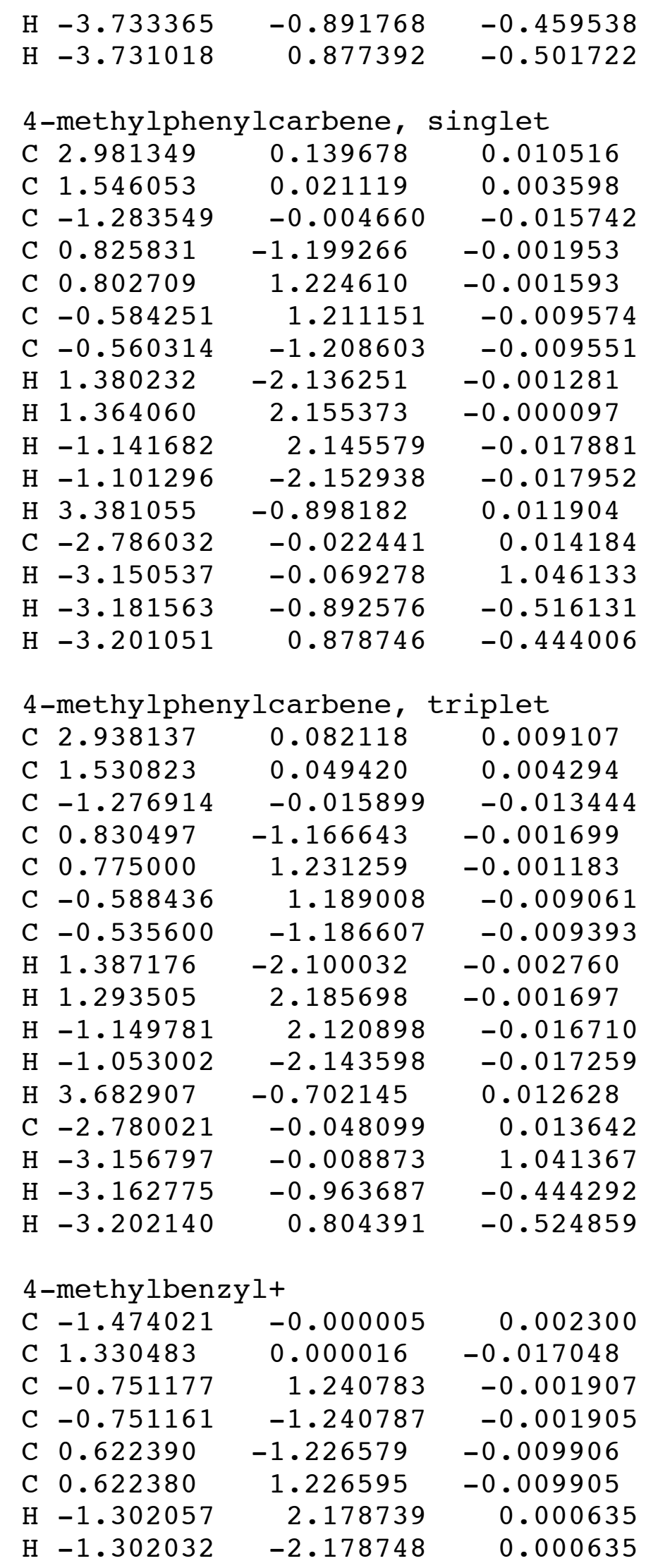




\begin{tabular}{|c|c|c|c|}
\hline $\mathrm{H}$ & 1.178755 & -2.159768 & -0.016695 \\
\hline $\mathrm{H}$ & 1.178729 & 2.159793 & -0.016696 \\
\hline & 2.820060 & 0.000000 & 0.010784 \\
\hline & 3.158501 & -0.000446 & 1.055581 \\
\hline & 3.230399 & 0.891574 & -0.467852 \\
\hline & 3.230349 & -0.891210 & -0.468584 \\
\hline & -2.843752 & -0.000008 & 0.008979 \\
\hline & -3.411925 & -0.927159 & 0.012313 \\
\hline & -3.411930 & 0.927138 & 0.012311 \\
\hline \multicolumn{4}{|c|}{ 4-methyltoluene } \\
\hline & -1.417640 & -0.000012 & 0.019718 \\
\hline & 1.417640 & 0.000042 & -0.019684 \\
\hline & -0.696692 & 1.198314 & 0.007021 \\
\hline & -0.696640 & -1.198323 & 0.007031 \\
\hline & 0.696680 & -1.198298 & -0.007070 \\
\hline C & 0.696647 & 1.198341 & -0.007058 \\
\hline & -1.232910 & 2.146045 & 0.015521 \\
\hline $\mathrm{H}$ & -1.232841 & -2.146064 & 0.015551 \\
\hline $\mathrm{H}$ & 1.232913 & -2.146023 & -0.015589 \\
\hline & 1.232828 & 2.146091 & -0.015548 \\
\hline $\mathrm{C}$ & 2.922485 & -0.000016 & 0.007304 \\
\hline $\mathrm{H}$ & 3.301052 & -0.000298 & 1.035096 \\
\hline & 3.325365 & 0.884993 & -0.492907 \\
\hline $\mathrm{H}$ & 3.325348 & -0.884787 & -0.493358 \\
\hline $\mathrm{C}$ & -2.922482 & -0.000025 & -0.007273 \\
\hline $\mathrm{H}$ & -3.325327 & -0.884882 & 0.493257 \\
\hline $\mathrm{H}$ & -3.301052 & -0.000114 & -1.035057 \\
\hline & -3.325366 & 0.884897 & 0.493101 \\
\hline \multicolumn{4}{|c|}{ 4-fluoromethylphenylcarbene, singlet } \\
\hline $\mathrm{C}$ & -3.396074 & -0.220745 & -0.000416 \\
\hline C & -1.957917 & -0.116923 & -0.000104 \\
\hline $\mathrm{C}$ & 0.826122 & 0.284844 & 0.000379 \\
\hline $\mathrm{C}$ & -1.061170 & -1.212128 & 0.000403 \\
\hline $\mathrm{C}$ & -1.410328 & 1.188538 & -0.000386 \\
\hline $\mathrm{C}$ & -0.039063 & 1.388071 & -0.000162 \\
\hline C & 0.313469 & -1.017050 & 0.000714 \\
\hline $\mathrm{H}$ & -1.467467 & -2.222146 & 0.000591 \\
\hline $\mathrm{H}$ & -2.108296 & 2.021561 & -0.000865 \\
\hline $\mathrm{H}$ & 0.369188 & 2.397671 & -0.000521 \\
\hline $\mathrm{H}$ & 0.999351 & -1.857367 & 0.001040 \\
\hline C & 2.310536 & 0.523510 & 0.001222 \\
\hline $\mathrm{H}$ & -3.630922 & -1.307386 & -0.000086 \\
\hline $\mathrm{F}$ & 3.011308 & -0.679748 & -0.001727 \\
\hline & 2.611344 & 1.086037 & 0.891875 \\
\hline & 2.611588 & 1.090664 & -0.886399 \\
\hline
\end{tabular}




\begin{tabular}{|c|c|c|c|}
\hline \multicolumn{4}{|c|}{ 4-fluoromethylphenylcarbene, triple } \\
\hline $\mathrm{C}$ & -3.324122 & -0.394699 & \\
\hline $\mathrm{C}$ & -1.935427 & -0.168782 & -0.013752 \\
\hline C & 0.819299 & 0.276893 & 0.068566 \\
\hline $\mathrm{C}$ & -1.410956 & 1.133273 & -0.050007 \\
\hline $\mathrm{C}$ & -1.028325 & -1.236115 & 0.060551 \\
\hline $\mathrm{C}$ & 0.317179 & -1.012374 & 0.105027 \\
\hline $\mathrm{C}$ & -0.062181 & 1.341120 & -0.012401 \\
\hline $\mathrm{H}$ & -2.090322 & 1.978562 & -0.117219 \\
\hline $\mathrm{H}$ & -1.414415 & -2.250942 & 0.083315 \\
\hline $\mathrm{H}$ & 1.007221 & -1.847937 & 0.155807 \\
\hline $\mathrm{H}$ & 0.321257 & 2.358961 & -0.053313 \\
\hline $\mathrm{C}$ & 2.297129 & 0.523917 & 0.164692 \\
\hline $\mathrm{H}$ & -4.168952 & 0.277996 & -0.115911 \\
\hline $\mathrm{F}$ & 3.012829 & -0.599979 & -0.252498 \\
\hline $\mathrm{H}$ & 2.598140 & 0.733902 & 1.197295 \\
\hline $\mathrm{H}$ & 2.596031 & 1.369872 & -0.462756 \\
\hline & fluoromet & lbenzylt & \\
\hline $\mathrm{C}$ & -0.481350 & -1.822049 & 0.000000 \\
\hline $\mathrm{C}$ & 0.000000 & 0.921817 & 0.000000 \\
\hline $\mathrm{C}$ & 0.865077 & -1.324732 & 0.000000 \\
\hline $\mathrm{C}$ & -1.582652 & -0.899468 & 0.000000 \\
\hline $\mathrm{C}$ & -1.333437 & 0.451037 & 0.000000 \\
\hline $\mathrm{C}$ & 1.093691 & 0.032070 & 0.000000 \\
\hline $\mathrm{H}$ & 1.693216 & -2.030271 & 0.000000 \\
\hline $\mathrm{H}$ & -2.600676 & -1.282455 & 0.000000 \\
\hline $\mathrm{H}$ & -2.157886 & 1.160207 & 0.000000 \\
\hline $\mathrm{H}$ & 2.100900 & 0.433073 & 0.000000 \\
\hline $\mathrm{C}$ & 0.247095 & 2.399987 & 0.000000 \\
\hline $\mathrm{F}$ & 1.602375 & 2.662489 & 0.000000 \\
\hline $\mathrm{H}$ & -0.205120 & 2.854382 & 0.890218 \\
\hline $\mathrm{H}$ & -0.205120 & 2.854382 & -0.890218 \\
\hline $\mathrm{C}$ & -0.713230 & -3.172918 & 0.000000 \\
\hline $\mathrm{H}$ & 0.105113 & -3.889225 & 0.000000 \\
\hline $\mathrm{H}$ & -1.722966 & -3.576963 & 0.000000 \\
\hline & fluoromet & Itoluene & \\
\hline $\mathrm{C}$ & -1.833828 & -0.085592 & 0.004975 \\
\hline $\mathrm{C}$ & 0.961859 & 0.273525 & 0.075868 \\
\hline $\mathrm{C}$ & -0.967790 & -1.181715 & 0.084456 \\
\hline C & -1.273217 & 1.194911 & -0.046058 \\
\hline $\mathrm{C}$ & 0.107931 & 1.375019 & -0.011184 \\
\hline $\mathrm{C}$ & 0.413443 & -1.009243 & 0.130277 \\
\hline $\mathrm{H}$ & -1.382176 & -2.187809 & 0.122674 \\
\hline $\mathrm{H}$ & -1.925869 & 2.063560 & -0.114190 \\
\hline $\mathrm{H}$ & 0.520842 & 2.381712 & -0.061440 \\
\hline $\mathrm{H}$ & 1.073019 & -1.869967 & 0.188595 \\
\hline
\end{tabular}




\begin{tabular}{|c|c|c|c|}
\hline & 2.446291 & 0.471028 & 0.180045 \\
\hline $\mathrm{F}$ & 3.126737 & -0.653280 & -0.296271 \\
\hline $\mathrm{H}$ & 2.757172 & 0.619033 & 1.220356 \\
\hline & 2.770154 & 1.337287 & -0.406315 \\
\hline & -3.323503 & -0.281795 & -0.079995 \\
\hline & -3.858511 & 0.573946 & 0.340683 \\
\hline & -3.633972 & -1.177296 & 0.465189 \\
\hline & -3.648408 & -0.397779 & -1.119411 \\
\hline \multicolumn{3}{|c|}{ 4-fluorophenylcarbene, } & singlet \\
\hline $\mathrm{C}$ & 2.919686 & 0.144188 & -0.000004 \\
\hline & 1.485773 & 0.021681 & 0.000001 \\
\hline $\mathrm{C}$ & -1.291157 & -0.006658 & 0.000000 \\
\hline $\mathrm{C}$ & 0.769760 & -1.203083 & 0.000002 \\
\hline C & 0.743307 & 1.227876 & 0.000001 \\
\hline $\mathrm{C}$ & -0.642983 & 1.225341 & 0.000001 \\
\hline C & -0.615688 & -1.226050 & 0.000000 \\
\hline & 1.326608 & -2.137828 & 0.000003 \\
\hline $\mathrm{H}$ & 1.306039 & 2.157132 & -0.000002 \\
\hline $\mathrm{H}$ & -1.230805 & 2.137666 & 0.000002 \\
\hline $\mathrm{H}$ & -1.185911 & -2.149675 & -0.000002 \\
\hline $\mathrm{F}$ & -2.639152 & -0.024004 & -0.000001 \\
\hline $\mathrm{H}$ & 3.324245 & -0.891030 & 0.000001 \\
\hline \multicolumn{3}{|c|}{ 4-fluorophenylcarbene, } & triplet \\
\hline $\mathrm{C}$ & 0.047159 & -2.882421 & 0.000000 \\
\hline $\mathrm{C}$ & 0.030024 & -1.472139 & 0.000000 \\
\hline C & 0.000000 & 1.287130 & 0.000000 \\
\hline $\mathrm{C}$ & -1.178866 & -0.759742 & 0.000000 \\
\hline C & 1.222423 & -0.733960 & 0.000000 \\
\hline $\mathrm{C}$ & 1.209846 & 0.631319 & 0.000000 \\
\hline C & -1.194839 & 0.607036 & 0.000000 \\
\hline $\mathrm{H}$ & -2.117673 & -1.306070 & 0.000000 \\
\hline $\mathrm{H}$ & 2.168983 & -1.265591 & 0.000000 \\
\hline $\mathrm{H}$ & 2.128417 & 1.208387 & 0.000000 \\
\hline $\mathrm{H}$ & -2.125627 & 1.164283 & 0.000000 \\
\hline $\mathrm{F}$ & -0.013750 & 2.639364 & 0.000000 \\
\hline $\mathrm{H}$ & -0.744826 & -3.618621 & 0.000000 \\
\hline \multicolumn{4}{|c|}{ 4-fluorobenzyl+ } \\
\hline $\mathrm{C}$ & 0.000000 & -1.412152 & 0.000000 \\
\hline $\mathrm{C}$ & 0.000000 & 1.339199 & 0.000000 \\
\hline C & 1.245606 & -0.690702 & 0.000000 \\
\hline $\mathrm{C}$ & -1.245606 & -0.690702 & 0.000000 \\
\hline $\mathrm{C}$ & -1.244284 & 0.680471 & 0.000000 \\
\hline $\mathrm{C}$ & 1.244284 & 0.680471 & 0.000000 \\
\hline $\mathrm{H}$ & 2.182012 & -1.243602 & 0.000000 \\
\hline & -2.182012 & -1.243602 & 0.000000 \\
\hline
\end{tabular}




\begin{tabular}{|c|c|c|c|}
\hline & -2.156311 & 1.268890 & 0.000000 \\
\hline & 2.156311 & 1.268890 & 0.000000 \\
\hline & 0.000000 & 2.654553 & 0.000000 \\
\hline & 0.000000 & -2.780529 & 0.000000 \\
\hline & 0.927007 & -3.348943 & 0.000000 \\
\hline & -0.927007 & -3.348943 & 0.000000 \\
\hline \multicolumn{4}{|c|}{ 4-fluorotoluene } \\
\hline & -1.360856 & 0.000027 & -0.016387 \\
\hline & 1.423695 & -0.000008 & 0.004168 \\
\hline & -0.642762 & 1.201293 & -0.009283 \\
\hline & -0.642790 & -1.201257 & -0.009293 \\
\hline & 0.751061 & -1.213470 & -0.002097 \\
\hline & 0.751087 & 1.213471 & -0.002105 \\
\hline & -1.180260 & 7433 & -0.017648 \\
\hline & -1.180301 & -2.147390 & -0.017675 \\
\hline & 1.315448 & -2.140844 & -0.002269 \\
\hline & 1.315501 & 2.14 & -0.002 \\
\hline $\mathrm{F}$ & 2.781003 & -0.00 & 0.009020 \\
\hline & -2.865862 & -0.000014 & 0.015489 \\
\hline & -3.270 & 0.8 & $-0.4 \varepsilon$ \\
\hline & -3.240851 & -0.000188 & 1.044329 \\
\hline & -3.269994 & -0.884350 & -0.484418 \\
\hline \multicolumn{4}{|c|}{ 4-chlorophenylcarbene, singlet } \\
\hline & 0.137340 & -3.360880 & 0.000000 \\
\hline $\mathrm{C}$ & 0.019703 & -1.92 & 0.000 \\
\hline $\mathrm{C}$ & 0.000000 & 0.871900 & 0.000000 \\
\hline & -1.200581 & -1.203363 & 0.000000 \\
\hline $\mathrm{C}$ & 1.226172 & -1.185030 & 0.000000 \\
\hline $\mathrm{C}$ & 1.226544 & 0.201906 & 0.000000 \\
\hline $\mathrm{C}$ & -1.217896 & 0.182722 & 0.000000 \\
\hline $\mathrm{H}$ & -2.138699 & -1.755012 & 0.000000 \\
\hline $\mathrm{H}$ & 2.155561 & -1.748124 & 0.000000 \\
\hline $\mathrm{H}$ & 2.151496 & 0.770597 & 0.000000 \\
\hline & -2.150492 & 0.739081 & 0.000000 \\
\hline $\mathrm{H}$ & -0.899342 & -3.761363 & 0.000000 \\
\hline & -0.015660 & 2.603246 & 0.00000 \\
\hline
\end{tabular}

4-chlorophenylcarbene, triplet
C 0.060185
$-3.315121$
0.000000
C 0.039040
$-1.909073$
0.000000
C 0.000000
0.865320
0.000000
C -1.173129
$-1.199846$
0.000000
C 1.229897
$-1.166532$
0.000000
C 1.209247
0.197355
0.000000
C -1.189649
0.165414
0.000000
$\mathrm{H}-2.111001$
$-1.747952$
0.000000 


\begin{tabular}{|c|c|c|}
\hline Н 2.179023 & -1.693770 & 0.000000 \\
\hline H 2.135923 & 0.762315 & 0.000000 \\
\hline-2.131802 & 0.704301 & 0.000000 \\
\hline-0.727793 & -4.055574 & 0.000000 \\
\hline $1-0.023406$ & 2.600328 & 0.00000 \\
\hline \multicolumn{3}{|c|}{ 4-chlorobenzyl+ } \\
\hline C 0.000000 & -1.847089 & 0.000000 \\
\hline C 0.000000 & 0.933095 & 0.000000 \\
\hline C 1.242137 & -1.124230 & 0.000000 \\
\hline C -1.242137 & -1.124230 & 0.000000 \\
\hline$C-1.237790$ & 0.247663 & 0.000000 \\
\hline C 1.237790 & 0.247663 & 0.0 \\
\hline H 2.180602 & -1.674147 & 0.000000 \\
\hline $\mathrm{H}-2.180602$ & -1.674147 & 0.000000 \\
\hline $\mathrm{H}-2.162699$ & 0.816 & 0000 \\
\hline H 2.162699 & 0.816327 & 0.000000 \\
\hline Cl 0.000000 & 2.622786 & 0.000000 \\
\hline C 0.000000 & -3.216 & 0.0 \\
\hline Н 0.927164 & -3.784789 & 0.00 \\
\hline $\mathrm{H}-0.927164$ & -3.784789 & 0.000000 \\
\hline \multicolumn{3}{|c|}{ 4-chlorotoluene } \\
\hline$C-1.802478$ & 0.000003 & -0.015865 \\
\hline C 0.998512 & 0.000001 & 0.0 \\
\hline C -1.082904 & -1.199578 & -0.009541 \\
\hline$C-1.082904$ & 1.199581 & -0.009542 \\
\hline C 0.310312 & 1.210684 & -0.0 \\
\hline C 0.310309 & -1.210683 & -0.003810 \\
\hline $\mathrm{H}-1.618456$ & -2.147029 & -0.017489 \\
\hline $\mathrm{H}-1.618453$ & 2.147033 & -0.017490 \\
\hline $\mathrm{H} \quad 0.859664$ & 2.147616 & -0.004112 \\
\hline $\mathrm{H} \quad 0.859661$ & -2.147615 & -0.004113 \\
\hline Cl 2.738592 & -0.000002 & 0.005976 \\
\hline$C-3.306830$ & -0.000002 & 0.017185 \\
\hline-3.679455 & -0.000150 & 1.046952 \\
\hline $\mathrm{H}-3.711561$ & -0.884649 & -0.481611 \\
\hline $\mathrm{H}-3.711566$ & 0.884784 & -0.481360 \\
\hline \multicolumn{3}{|c|}{ 4-formylphenylcarbene, singlet } \\
\hline C 3.322536 & -0.142979 & 0.000000 \\
\hline C 1.876554 & -0.088729 & 0.000000 \\
\hline C -0.907749 & 0.223966 & 0.000000 \\
\hline C 1.018485 & -1.218867 & 0.000000 \\
\hline C 1.293708 & 1.200292 & 0.000000 \\
\hline$C-0.086091$ & 1.358099 & 0.000000 \\
\hline$C-0.358882$ & -1.067665 & 0.000000 \\
\hline Н 1.461527 & -2.213070 & 0.000000 \\
\hline
\end{tabular}




\begin{tabular}{|c|c|c|c|}
\hline $\mathrm{H}$ & 1.968847 & 2.051801 & 0.000000 \\
\hline $\mathrm{H}$ & -0.534669 & 2.350130 & 0.000000 \\
\hline & -1.033722 & -1.919527 & 0.000000 \\
\hline & -2.382629 & 0.388307 & 0.000000 \\
\hline & -3.170941 & -0.549951 & 0.000000 \\
\hline & -2.745872 & 1.435779 & 0.000000 \\
\hline & 3.595823 & -1.220052 & 0.000000 \\
\hline \multicolumn{4}{|c|}{ 4-formylphenylcarbene, triplet } \\
\hline & -3.257302 & -0.195806 & -0.000002 \\
\hline & -1.860356 & -0.056391 & -0.000001 \\
\hline C & 0.892969 & 0.214415 & 0.000003 \\
\hline & -1.024324 & -1.192386 & 0.000002 \\
\hline $\mathrm{C}$ & -1.267666 & 1.216142 & -0.000002 \\
\hline C & 0.092055 & 1.338366 & 0.000000 \\
\hline $\mathrm{C}$ & 0.327599 & -1.050591 & 0.000003 \\
\hline $\mathrm{H}$ & -1.475136 & -2.180741 & 0.000003 \\
\hline & -1.906080 & 2.094097 & -0.000004 \\
\hline $\mathrm{H}$ & 0.548350 & 2.326645 & 0.000000 \\
\hline $\mathrm{H}$ & 0.988834 & -1.912361 & 0.000004 \\
\hline C & 2.386115 & 0.351726 & 0.000003 \\
\hline 0 & 3.160605 & -0.554152 & -0.000 \\
\hline $\mathrm{H}$ & 2.728936 & 1.414914 & 0.000014 \\
\hline & -3.904281 & -1.062193 & -0.000002 \\
\hline \multicolumn{4}{|c|}{ 4-formylbenzyl+ } \\
\hline C & -1.801476 & -0.101193 & -0.000001 \\
\hline C & 0.950935 & 0.245760 & 0.000005 \\
\hline $\mathrm{C}$ & -0.935801 & -1.247395 & 0.000002 \\
\hline $\mathrm{C}$ & -1.249112 & 1.224416 & -0.000001 \\
\hline C & 0.119515 & 1.383712 & 0.000002 \\
\hline $\mathrm{C}$ & 0.428731 & -1.063893 & 0.000006 \\
\hline $\mathrm{H}$ & -1.370272 & -2.244786 & 0.000003 \\
\hline $\mathrm{H}$ & -1.917683 & 2.082613 & -0.000003 \\
\hline $\mathrm{H}$ & 0.561022 & 2.377627 & 0.000002 \\
\hline $\mathrm{H}$ & 1.123075 & -1.899665 & 0.000009 \\
\hline C & 2.445649 & 0.407781 & 0.000004 \\
\hline $\mathrm{O}$ & 3.182667 & -0.566876 & -0.000009 \\
\hline $\mathrm{H}$ & 2.832460 & 1.441360 & -0.000001 \\
\hline $\mathrm{C}$ & -3.164058 & -0.271919 & -0.000005 \\
\hline $\mathrm{H}$ & -3.612359 & -1.263111 & -0.000003 \\
\hline $\mathrm{H}$ & -3.843883 & 0.577364 & -0.000006 \\
\hline \multicolumn{4}{|c|}{ 4-formyltoluene } \\
\hline C & -1.751274 & -0.069721 & -0.014973 \\
\hline C & 1.040927 & 0.219326 & 0.002279 \\
\hline C & -0.912100 & -1.194225 & -0.009916 \\
\hline & -1.166030 & 1.201105 & -0.008134 \\
\hline
\end{tabular}




$\begin{array}{cccc}\mathrm{C} & 0.218684 & 1.348810 & -0.002185 \\ \mathrm{C} & 0.469852 & -1.058403 & -0.003747 \\ \mathrm{H} & -1.355242 & -2.188479 & -0.018561 \\ \mathrm{H} & -1.803170 & 2.083356 & -0.015265 \\ \mathrm{H} & 0.663701 & 2.342979 & -0.001506 \\ \mathrm{H} & 1.125899 & -1.925078 & -0.004079 \\ \mathrm{C} & 2.508775 & 0.376221 & 0.005399 \\ \mathrm{O} & 3.304467 & -0.557281 & 0.005451 \\ \mathrm{H} & 2.871385 & 1.425656 & 0.008543 \\ \mathrm{C} & -3.246507 & -0.231953 & 0.015638 \\ \mathrm{H} & -3.604099 & -0.380724 & 1.040086 \\ \mathrm{H} & -3.560465 & -1.098672 & -0.572407 \\ \mathrm{H} & -3.747717 & 0.652245 & -0.386592\end{array}$

4-trifluoromethylphenylcarbene, singlet
C 3.957299
0.109060
0.000426
C 2.513420
0.011449
0.000060
C -0.282306
0.035300
$-0.000694$
C 1.779232
$-1.200526$
$-0.000141$
C 1.797404
1.229567
$-0.000139$
C 0.407656
1.249092
$-0.000485$
C 0.392917
$-1.192354$
$-0.000531$
H 2.319917
$-2.144802$
$-0.000047$
H 2.377404
2.148373
$-0.000032$
$\mathrm{H}-0.140902$
2.185077
$-0.000710$
$\mathrm{H}-0.173509$
$-2.119862$
$-0.000859$
0.002447
0.000005
C -1.781032
$-0.644288$
1.090824
$-0.657885$
$-1.082254$
1.235525
$-0.007449$
F -2.326463
$-0.933177$
0.000545

4-trifluoromethylphenylcarbene, triplet
C 3.903004
0.061772
0.024274
C 2.496184
0.042217
0.005628
C -0.276161
0.005570
$-0.038028$
C 1.791609
$-1.172596$
$-0.007493$
C 1.760293
1.237190
$-0.006177$
C 0.397606
1.213752
$-0.026070$
C 0.427447
$-1.184005$
$-0.027179$
H 2.343425
$-2.108035$
$-0.003917$
H 2.293065
2.182949
$-0.001441$
$\mathrm{H}-0.162279$
2.143241
$\mathrm{H}-0.107935$
$-2.127831$
$-0.041539$
C -1.769086
$-0.012865$
$-0.043462$
F -2.241462
$-0.007764$
0.000559
$-1.115080$
1.270620
F -2.276685
1.066236
$-0.598193$
F -2.303424
$-0.616598$ 


\begin{tabular}{|c|c|c|c|}
\hline \multicolumn{2}{|c|}{ Н 4.642489} & -0.727065 & 0.034824 \\
\hline \multicolumn{4}{|c|}{ 4-trifluoromethylbenzyl+ } \\
\hline & -2.438655 & -0.000010 & 0.000728 \\
\hline & 0.328746 & 0.000058 & -0.045254 \\
\hline ᄃ & -1.724168 & 1.244197 & -0.013006 \\
\hline C & -1.724115 & -1.244185 & -0.012994 \\
\hline $\mathrm{C}$ & -0.346299 & -1.234370 & -0.036197 \\
\hline & -0.346354 & 1.234449 & -0.036174 \\
\hline $\mathrm{H}$ & -2.277642 & 2.180515 & -0.005270 \\
\hline $\mathrm{H}$ & -2.277542 & -2.180531 & -0.005253 \\
\hline $\mathrm{H}$ & 0.219734 & -2.160311 & -0.053040 \\
\hline $\mathrm{H}$ & 0.219645 & 2.160410 & -0.052998 \\
\hline & 1.844259 & 0.000018 & 0.001749 \\
\hline $\mathrm{F}$ & 2.234413 & -0.000475 & 1.285559 \\
\hline $\mathrm{F}$ & 2.337692 & 1.090615 & -0.601130 \\
\hline $\mathrm{F}$ & 2.337571 & -1.090223 & -0.601899 \\
\hline $\mathrm{C}$ & -3.811873 & -0.000033 & 0.024958 \\
\hline n & -4.380279 & 0.927451 & 0.035458 \\
\hline & -4.380250 & -0.927534 & 0.035480 \\
\hline
\end{tabular}

4-trifluoromethyltoluene
C 2.402490
0.000009
0.017985
C -0.400080
0.000005
$-0.046752$
C 1.685478
$-1.201572$
$-0.000822$
C 1.685476
1.201588
$-0.000830$
C 0.293756
1.210042
$-0.027322$
C 0.293759
$-1.210032$
$-0.027328$
$\mathrm{H} 2.224651$
$-2.146702$
0.012217
H 2.224649
2.146717
0.012204
$\mathrm{H}-0.250608$
2.149596
$-0.043288$
$\mathrm{H}-0.250599$
$-2.149589$
$-0.043297$
C -1.892422
$-0.000001$
$-0.001091$
F -2.353812
$-0.000002$
1.272591
F -2.417574
$-1.090011$
$-0.606835$
F -2.417596
1.089996
C 3.906916
$-0.000010$
$-0.606838$
H 4.292349
$-0.000491$
0.007383
$\mathrm{H} 4.304080$
$-0.884955$
$-1.017594$
0.511489
H 4.304081
0.885398
0.510675

4-cyanophenylcarbene, singlet
C -3.265683
0.133050
$-0.000015$
C -1.822916
0.016785
C 0.978712
0.000918
C -1.105066
$-1.204625$
$-0.000004$
C -1.090202
1.226893
0.000013
C 0.297128
1.229473
0.000003
0.000002
0.000010 


\begin{tabular}{|c|c|c|c|}
\hline $\mathrm{C}$ & 0.281538 & -1.220433 & 0.000010 \\
\hline $\mathrm{H}$ & -1.658769 & -2.141443 & 0.000000 \\
\hline $\mathrm{H}$ & -1.658364 & 2.153172 & -0.000003 \\
\hline $\mathrm{H}$ & 0.862606 & 2.157209 & 0.000016 \\
\hline & 0.837127 & -2.154292 & 0.000015 \\
\hline C & 2.412492 & -0.010214 & 0.000012 \\
\hline & 3.595132 & -0.020267 & -0.000028 \\
\hline & -3.664543 & -0.903856 & -0.000022 \\
\hline \multicolumn{4}{|c|}{ 4-cyanophenylcarbene, triplet } \\
\hline C & 0.058807 & -3.207119 & 0.000000 \\
\hline C & 0.037958 & -1.802134 & 0.000000 \\
\hline $\mathrm{C}$ & 0.000000 & 0.964400 & 0.000000 \\
\hline $\mathrm{C}$ & -1.179150 & -1.099304 & 0.000000 \\
\hline C & 1.234347 & -1.066733 & 0.000000 \\
\hline $\mathrm{C}$ & 1.211566 & 0.295459 & 0.000000 \\
\hline C & -1.192556 & 0.264096 & 0.000000 \\
\hline $\mathrm{H}$ & -2.113802 & -1.652032 & 0.000000 \\
\hline $\mathrm{H}$ & 2.179870 & -1.599541 & 0.000000 \\
\hline $\mathrm{H}$ & 2.138122 & 0.860974 & 0.000000 \\
\hline $\mathrm{H}$ & -2.134189 & 0.804265 & 0.000000 \\
\hline C & -0.019816 & 2.417556 & 0.000000 \\
\hline $\mathrm{N}$ & -0.035601 & 3.562568 & 0.000000 \\
\hline $\mathrm{H}$ & -0.727726 & -3.948970 & 0.00000 \\
\hline \multicolumn{4}{|c|}{ 4-cyanobenzyl+ } \\
\hline $\mathrm{C}$ & 0.000000 & -1.750747 & 0.000000 \\
\hline C & 0.000000 & 1.025545 & 0.000000 \\
\hline $\mathrm{C}$ & 1.244394 & -1.035456 & 0.000000 \\
\hline $\mathrm{C}$ & -1.244393 & -1.035456 & 0.000000 \\
\hline $\mathrm{C}$ & -1.239049 & 0.340914 & 0.000000 \\
\hline $\mathrm{C}$ & 1.239051 & 0.340914 & 0.000000 \\
\hline $\mathrm{H}$ & 2.181058 & -1.588693 & 0.000000 \\
\hline $\mathrm{H}$ & -2.181057 & -1.588693 & 0.000000 \\
\hline $\mathrm{H}$ & -2.164831 & 0.909075 & 0.000000 \\
\hline $\mathrm{H}$ & 2.164832 & 0.909075 & 0.000000 \\
\hline $\mathrm{C}$ & 0.000000 & 2.452637 & 0.000000 \\
\hline $\mathrm{N}$ & -0.000003 & 3.637426 & 0.000000 \\
\hline $\mathrm{C}$ & 0.000000 & -3.124496 & 0.000000 \\
\hline $\mathrm{H}$ & 0.927635 & -3.692935 & 0.000000 \\
\hline $\mathrm{H}$ & -0.927634 & -3.692935 & 0.000000 \\
\hline \multicolumn{4}{|c|}{ 4-cyanotoluene } \\
\hline $\mathrm{C}$ & -1.695532 & 0.000004 & -0.015517 \\
\hline $\mathrm{C}$ & 1.112384 & 0.000000 & 0.002044 \\
\hline $\mathrm{C}$ & -0.978189 & 1.202096 & -0.009398 \\
\hline $\mathrm{C}$ & -0.978191 & -1.202092 & -0.009398 \\
\hline C & 0.412788 & -1.213802 & -0.003346 \\
\hline
\end{tabular}




\begin{tabular}{|c|c|c|c|}
\hline C & 0.412793 & 1.213802 & -0.003346 \\
\hline $\mathrm{H}$ & -1.517603 & 2.147100 & -0.017278 \\
\hline 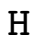 & -1.517607 & -2.147094 & -0.017282 \\
\hline 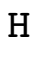 & 0.960375 & -2.152479 & -0.003322 \\
\hline $\mathrm{H}$ & 0.960382 & 2.152477 & -0.003325 \\
\hline $\mathrm{C}$ & 2.545786 & -0.000002 & 0.004984 \\
\hline N & 3.728125 & -0.000003 & 0.008149 \\
\hline C & -3.199369 & -0.000003 & 0.016955 \\
\hline $\mathrm{H}$ & -3.603972 & 0.885234 & -0.480620 \\
\hline 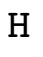 & -3.603982 & -0.885135 & -0.480798 \\
\hline \multicolumn{4}{|c|}{ 4-nitrophenylcarbene, singlet } \\
\hline $\mathrm{C}$ & 3.626243 & -0.134068 & -0.000002 \\
\hline C & 2.183243 & -0.017942 & 0.000000 \\
\hline $\mathrm{C}$ & -0.591498 & -0.002483 & 0.000000 \\
\hline $\mathrm{C}$ & 1.464023 & 1.202952 & 0.000008 \\
\hline $\mathrm{C}$ & 1.449332 & -1.227721 & -0.000008 \\
\hline $\mathrm{C}$ & 0.060650 & -1.234414 & -0.000007 \\
\hline $\mathrm{C}$ & 0.076205 & 1.222719 & 0.000007 \\
\hline $\mathrm{H}$ & 2.016535 & 2.140302 & 0.000014 \\
\hline $\mathrm{H}$ & 2.016352 & -2.154540 & -0.000014 \\
\hline $\mathrm{H}$ & -0.517327 & -2.151451 & -0.000012 \\
\hline $\mathrm{H}$ & -0.492430 & 2.145674 & 0.000012 \\
\hline $\mathrm{N}$ & -2.065248 & 0.008450 & 0.000000 \\
\hline $\mathrm{H}$ & 4.025023 & 0.902648 & 0.000009 \\
\hline 0 & -2.629503 & 1.114090 & -0.000019 \\
\hline O & -2.645572 & -1.088595 & 0.000019 \\
\hline \multicolumn{4}{|c|}{ 4-nitrophenylcarbene, triplet } \\
\hline $\mathrm{C}$ & -3.564984 & 0.066241 & 0.000000 \\
\hline C & -2.160076 & 0.043621 & 0.000000 \\
\hline $\mathrm{C}$ & 0.586657 & 0.002646 & 0.000000 \\
\hline $\mathrm{C}$ & -1.459872 & -1.176107 & 0.000004 \\
\hline $\mathrm{C}$ & -1.424354 & 1.240857 & -0.000005 \\
\hline $\mathrm{C}$ & -0.063512 & 1.220249 & -0.000004 \\
\hline $\mathrm{C}$ & -0.098054 & -1.194851 & 0.000004 \\
\hline $\mathrm{H}$ & -2.015284 & -2.109220 & 0.000009 \\
\hline $\mathrm{H}$ & -1.957667 & 2.186148 & -0.000009 \\
\hline $\mathrm{H}$ & 0.516446 & 2.135654 & -0.000008 \\
\hline $\mathrm{H}$ & 0.454863 & -2.126920 & 0.000007 \\
\hline $\mathrm{N}$ & 2.050952 & -0.019316 & 0.000000 \\
\hline $\mathrm{H}$ & -4.308347 & -0.718957 & 0.000003 \\
\hline 0 & 2.612315 & -1.126404 & -0.000011 \\
\hline $\mathrm{O}$ & 2.644995 & 1.070476 & 0.000012 \\
\hline \multicolumn{4}{|c|}{ 4-nitrobenzyl+ } \\
\hline $\mathrm{C}$ & 0.000000 & -2.110426 & 0.000000 \\
\hline & 0.000000 & 0.636853 & 0.000000 \\
\hline
\end{tabular}




$\begin{array}{llcc}\mathrm{C} & 1.245638 & -1.394420 & 0.000000 \\ \mathrm{C}-1.245638 & -1.394420 & 0.000000 \\ \mathrm{C}-1.241940 & -0.016489 & 0.000000 \\ \mathrm{C} & 1.241940 & -0.016489 & 0.000000 \\ \mathrm{H} & 2.182209 & -1.947888 & 0.000000 \\ \mathrm{H}-2.182209 & -1.947888 & 0.000000 \\ \mathrm{H}-2.155777 & 0.568457 & 0.000000 \\ \mathrm{H} & 2.155777 & 0.568456 & 0.000000 \\ \mathrm{~N} & 0.000000 & 2.118294 & 0.000000 \\ \mathrm{O} & 1.110343 & 2.669128 & 0.000000 \\ \mathrm{O}-1.110343 & 2.669128 & 0.000000 \\ \mathrm{C} & 0.000000 & -3.483146 & 0.000000 \\ \mathrm{H}-0.927545 & -4.052011 & 0.000000 \\ \mathrm{H} & 0.927544 & -4.052011 & 0.000000 \\ & & & \\ 4-\mathrm{nitrOtOluene} & & \\ \mathrm{C} & 2.063014 & 0.000044 & -0.015022 \\ \mathrm{C} & -0.717628 & 0.000007 & 0.000775 \\ \mathrm{C} & 1.345082 & 1.201896 & -0.009668 \\ \mathrm{C} & 1.345086 & -1.201865 & -0.009653 \\ \mathrm{C} & -0.046639 & -1.217664 & -0.004029 \\ \mathrm{C} & -0.046685 & 1.217653 & -0.004054 \\ \mathrm{H} & 1.883864 & 2.147064 & -0.017019 \\ \mathrm{H} & 1.883894 & -2.147017 & -0.017006 \\ \mathrm{H} & -0.607619 & -2.145290 & -0.004653 \\ \mathrm{H} & -0.607691 & 2.145264 & -0.004702 \\ \mathrm{~N}-2.184984 & -0.000007 & 0.003382 \\ \mathrm{O}-2.761919 & 1.099545 & 0.005039 \\ \mathrm{O}-2.761888 & -1.099572 & 0.004986 \\ \mathrm{C} & 3.566720 & -0.000020 & 0.017139 \\ \mathrm{H} & 3.971136 & 0.885668 & -0.479692 \\ \mathrm{H} & 3.971222 & -0.884639 & -0.481503 \\ \mathrm{H} & 3.936837 & -0.001093 & 1.047773\end{array}$

\section{nonbenzenoid arylcarbene sets}

tropylcarbene+, singlet, (cycloheptatrienylcarbene) +
C -2.676360
$-0.000041$
$-0.117112$
C -1.285377
$-0.000026$
0.004767
C -0.651871
$-1.276772$
$-0.018333$
C -0.651912
1.276743
$-0.018280$
C 0.699943
1.563068
0.000582
$\mathrm{H}-1.315812$
2.140303
$-0.048751$
$\mathrm{H}-1.315747$
$-2.140345$
$-0.048913$
C 0.699992
$-1.563057$
0.000640
$\mathrm{H} 0.938513$
2.624915
0.003232
$\mathrm{H} \quad 0.938594$
$-2.624896$
0.003289
C 1.808681
$-0.692467$
0.013775 


\begin{tabular}{|c|c|c|c|}
\hline $\mathrm{H}$ & 2.783901 & -1.173990 & 0.022562 \\
\hline & 1.808659 & 0.692514 & 0.013738 \\
\hline $\mathrm{H}$ & 2.783864 & 1.174068 & 0.022499 \\
\hline & -3.323842 & 0.000165 & 0.767428 \\
\hline \multicolumn{4}{|c|}{ tropylcarbenet, singlet, } \\
\hline C & -1.301362 & 0.000000 & -0.000025 \\
\hline & -0.647727 & -1.304340 & 0.000178 \\
\hline & -0.647727 & 1.304340 & 0.000179 \\
\hline & 0.693140 & 1.560978 & 0.000103 \\
\hline & -1.306175 & 2.169427 & 0.000373 \\
\hline $\mathrm{H}$ & -1.306175 & -2.169427 & 0.000373 \\
\hline $\mathrm{C}$ & 0.693140 & -1.560978 & 0.000103 \\
\hline $\mathrm{H}$ & 0.934300 & 2.622382 & 0.000250 \\
\hline $\mathrm{H}$ & 0.934300 & -2.622382 & 0.000251 \\
\hline $\mathrm{C}$ & 1.818452 & -0.684006 & -0.000145 \\
\hline $\mathrm{H}$ & 2.788786 & -1.173988 & -0.000296 \\
\hline C & 1.818452 & 0.684006 & -0.000146 \\
\hline $\mathrm{H}$ & 2.788786 & 1.173988 & -0.000297 \\
\hline C & -2.616138 & 0.000000 & -0.000272 \\
\hline $\mathrm{H}$ & -3.695200 & 0.000000 & -0.000503 \\
\hline \multicolumn{4}{|c|}{ tropylcarbenet, triplet } \\
\hline $\mathrm{C}$ & 2.671630 & -0.140342 & 0.000009 \\
\hline C & 1.265007 & -0.068748 & 0.000001 \\
\hline $\mathrm{C}$ & 0.578489 & -1.300067 & -0.000005 \\
\hline $\mathrm{C}$ & 0.689808 & 1.216459 & -0.000006 \\
\hline C & -0.622742 & 1.568518 & -0.000006 \\
\hline $\mathrm{H}$ & 1.402045 & 2.039443 & -0.000011 \\
\hline $\mathrm{H}$ & 1.224640 & -2.175606 & -0.000009 \\
\hline $\mathrm{C}$ & -0.759695 & -1.535629 & -0.000005 \\
\hline $\mathrm{H}$ & -0.811863 & 2.639902 & -0.000011 \\
\hline $\mathrm{H}$ & -1.044623 & -2.585416 & -0.000012 \\
\hline $\mathrm{C}$ & -1.832718 & -0.616320 & 0.000006 \\
\hline $\mathrm{H}$ & -2.825744 & -1.059117 & 0.000016 \\
\hline $\mathrm{C}$ & -1.770927 & 0.752401 & 0.000005 \\
\hline $\mathrm{H}$ & -2.719355 & 1.284047 & 0.000017 \\
\hline $\mathrm{H}$ & 3.461793 & 0.599120 & 0.000017 \\
\hline \multicolumn{3}{|c|}{ tropylcarbenet, triplet, } & $\mathrm{C} 2 \mathrm{v}, \mathrm{ts}$ \\
\hline $\mathrm{C}$ & 0.000000 & 0.000000 & 1.271561 \\
\hline $\mathrm{C}$ & 0.000000 & 1.262120 & 0.630164 \\
\hline $\mathrm{C}$ & 0.000000 & -1.262120 & 0.630164 \\
\hline $\mathrm{C}$ & 0.000000 & -1.554385 & -0.697152 \\
\hline $\mathrm{H}$ & 0.000000 & -2.111231 & 1.310037 \\
\hline $\mathrm{H}$ & 0.000000 & 2.111231 & 1.310037 \\
\hline C & 0.000000 & 1.554385 & -0.697152 \\
\hline & 0.000000 & -2.615946 & -0.934596 \\
\hline
\end{tabular}




\begin{tabular}{|c|c|c|c|}
\hline $\mathrm{H}$ & 0.000000 & 2.615946 & -0.934596 \\
\hline C & 0.000000 & 0.686045 & -1.808030 \\
\hline $\mathrm{H}$ & 0.000000 & 1.173029 & -2.780207 \\
\hline $\mathrm{C}$ & 0.000000 & -0.686045 & -1.808030 \\
\hline & 0.000000 & -1.173029 & -2.780207 \\
\hline C & 0.000000 & 0.000000 & 2.658024 \\
\hline & 0.000000 & 0.000000 & 3.732225 \\
\hline \multicolumn{4}{|c|}{ tropylCH2++ } \\
\hline $\mathrm{H}$ & 2.841584 & 1.174726 & -0.000027 \\
\hline & 1.856364 & 0.707250 & -0.000028 \\
\hline C & 1.856364 & -0.707250 & 0.000028 \\
\hline $\mathrm{C}$ & 0.766986 & 1.594962 & -0.000019 \\
\hline C & -0.598024 & 1.285693 & 0.000022 \\
\hline $\mathrm{H}$ & 1.015711 & 2.655702 & -0.000010 \\
\hline $\mathrm{H}$ & 2.841584 & -1.174726 & 0.000026 \\
\hline C & 0.766986 & -1.594962 & 0.000022 \\
\hline $\mathrm{C}$ & -1.217187 & 0.000000 & 0.000000 \\
\hline $\mathrm{H}$ & -1.274183 & 2.141770 & 0.000064 \\
\hline C & -0.598024 & -1.285693 & -0.000026 \\
\hline $\mathrm{H}$ & 1.015711 & -2.655702 & 0.000013 \\
\hline C & -2.627471 & 0.000000 & 0.000001 \\
\hline $\mathrm{H}$ & -1.274183 & -2.141770 & -0.000067 \\
\hline $\mathrm{H}$ & -3.201097 & -0.928033 & -0.000051 \\
\hline $\mathrm{H}$ & -3.201097 & 0.928032 & 0.000057 \\
\hline \multicolumn{4}{|c|}{ tropylCH $2++$, perp ts } \\
\hline C & -0.602845 & -1.278619 & -0.000001 \\
\hline $\mathrm{H}$ & -1.264962 & -2.147059 & -0.000002 \\
\hline C & 0.766599 & -1.577460 & -0.000001 \\
\hline $\mathrm{C}$ & -1.186556 & 0.000000 & 0.000000 \\
\hline $\mathrm{C}$ & -0.602845 & 1.278619 & 0.000000 \\
\hline $\mathrm{C}$ & -2.652339 & 0.000000 & 0.000001 \\
\hline $\mathrm{H}$ & 1.004577 & -2.640605 & -0.000002 \\
\hline C & 1.862551 & -0.701017 & 0.000001 \\
\hline $\mathrm{H}$ & 2.842496 & -1.177162 & 0.000002 \\
\hline $\mathrm{C}$ & 1.862551 & 0.701017 & 0.000001 \\
\hline $\mathrm{H}$ & -1.264962 & 2.147059 & -0.000001 \\
\hline $\mathrm{C}$ & 0.766599 & 1.577460 & -0.000001 \\
\hline $\mathrm{H}$ & 1.004577 & 2.640605 & -0.000002 \\
\hline $\mathrm{H}$ & 2.842495 & 1.177162 & 0.000002 \\
\hline $\mathrm{H}$ & -3.223253 & -0.000006 & 0.937320 \\
\hline $\mathrm{H}$ & -3.223255 & 0.000006 & -0.937316 \\
\hline \multicolumn{4}{|c|}{ tropylCH3+ } \\
\hline & -2.910510 & 1.176654 & 0.006295 \\
\hline C & -1.933540 & 0.697917 & 0.003097 \\
\hline & -0.830813 & 1.557558 & 0.001521 \\
\hline
\end{tabular}




\begin{tabular}{|c|c|c|c|}
\hline C & -1.933554 & -0.697888 & 0.003121 \\
\hline C & -0.830844 & -1.557553 & 0.001552 \\
\hline $\mathrm{H}$ & -2.910535 & -1.176602 & 0.006360 \\
\hline $\mathrm{H}$ & -1.064878 & 2.620361 & 0.005667 \\
\hline $\mathrm{C}$ & 0.531080 & 1.249195 & -0.007272 \\
\hline $\mathrm{C}$ & 0.531052 & -1.249213 & -0.007314 \\
\hline & -1.064929 & -2.620352 & 0.005702 \\
\hline $\mathrm{C}$ & 1.177763 & -0.000012 & -0.017018 \\
\hline $\mathrm{H}$ & 1.194200 & 2.112998 & -0.011329 \\
\hline C & 2.681393 & -0.000002 & 0.008206 \\
\hline $\mathrm{H}$ & 1.194154 & -2.113029 & -0.011440 \\
\hline $\mathrm{H}$ & 3.087968 & 0.886658 & -0.481713 \\
\hline $\mathrm{H}$ & 3.087896 & -0.886644 & -0.481804 \\
\hline $\mathrm{H}$ & 3.031409 & -0.000070 & 1.046896 \\
\hline \multicolumn{3}{|c|}{ cyclopentadienylcarbene- } & singlet \\
\hline $\mathrm{C}$ & -2.215191 & -0.178464 & 0.000006 \\
\hline C & -0.834527 & -0.040090 & -0.000002 \\
\hline $\mathrm{C}$ & 0.017055 & 1.145560 & -0.000014 \\
\hline $\mathrm{C}$ & 0.096521 & -1.161067 & -0.000008 \\
\hline $\mathrm{H}$ & -0.363547 & 2.165717 & 0.000018 \\
\hline $\mathrm{C}$ & 1.335640 & 0.759595 & 0.000009 \\
\hline $\mathrm{C}$ & 1.385312 & -0.687693 & 0.000002 \\
\hline $\mathrm{H}$ & -0.229369 & -2.198049 & 0.000009 \\
\hline $\mathrm{H}$ & 2.299809 & -1.278847 & 0.000008 \\
\hline $\mathrm{H}$ & 2.207040 & 1.413357 & 0.000000 \\
\hline $\mathrm{H}$ & -2.622796 & 0.870771 & 0.000005 \\
\hline \multicolumn{3}{|c|}{ cyclopentadienylcarbene- } & triplet \\
\hline $\mathrm{C}$ & 2.222947 & -0.000037 & -0.100658 \\
\hline $\mathrm{C}$ & 0.802154 & -0.000014 & -0.025949 \\
\hline C & -0.055694 & 1.142174 & -0.017401 \\
\hline C & -0.055722 & -1.142180 & -0.017382 \\
\hline $\mathrm{H}$ & 0.290393 & 2.173772 & -0.029439 \\
\hline $\mathrm{C}$ & -1.377277 & 0.713255 & 0.020459 \\
\hline C & -1.377296 & -0.713228 & 0.020460 \\
\hline $\mathrm{H}$ & 0.290332 & -2.173790 & -0.029390 \\
\hline $\mathrm{H}$ & -2.261101 & -1.348850 & 0.049846 \\
\hline $\mathrm{H}$ & -2.261066 & 1.348897 & 0.049927 \\
\hline & 2.986767 & 0.000147 & 0.681886 \\
\hline
\end{tabular}

$\begin{array}{lcc}\text { fulvene } & & \\ \text { C }-0.753883 & 0.000000 & 0.000001 \\ \text { C } 0.121629 & 1.176119 & 0.000000 \\ \text { C } 0.121629 & -1.176119 & 0.000000 \\ \text { H }-0.225329 & 2.203267 & 0.000002 \\ \text { C } 1.405490 & 0.733884 & -0.000001 \\ \text { C } 1.405490 & -0.733884 & 0.000000\end{array}$




\begin{tabular}{|c|c|c|c|}
\hline $\mathrm{H}$ & -0.225329 & -2.203267 & 0.000003 \\
\hline $\mathrm{H}$ & 2.298471 & -1.350102 & -0.000001 \\
\hline & 2.298471 & 1.350102 & 0.000000 \\
\hline & -2.101499 & 0.000000 & -0.000001 \\
\hline & -2.669710 & -0.925919 & -0.000001 \\
\hline & -2.669710 & 0.925919 & -0.000001 \\
\hline \multicolumn{4}{|c|}{ fulvene, er pts } \\
\hline & 0.000000 & 0.000000 & 0.745632 \\
\hline & 0.000000 & 1.167247 & -0.111592 \\
\hline & 0.000000 & -1.167247 & -0.111592 \\
\hline & 0.000000 & 2.184737 & 0.256265 \\
\hline C & 0.000000 & 0.697466 & -1.430929 \\
\hline $\mathrm{C}$ & 0.000000 & -0.697466 & -1.430929 \\
\hline & 0.000000 & -2.184737 & 0.256265 \\
\hline $\mathrm{H}$ & 0.000000 & -1.352824 & -2.295548 \\
\hline & 0.000000 & 1.352824 & -2.295548 \\
\hline $\mathrm{C}$ & 0.000000 & 0.000000 & 2.110658 \\
\hline $\mathrm{H}$ & 0.914221 & 0.000000 & 2.725542 \\
\hline & -0.914221 & 0.000000 & 2.725542 \\
\hline \multicolumn{4}{|c|}{ methylcyclopentadienide- } \\
\hline $\mathrm{C}$ & 0.663567 & -0.000003 & -0.030782 \\
\hline $\mathrm{C}$ & -0.172599 & 1.138790 & -0.011042 \\
\hline $\mathrm{C}$ & -0.172604 & -1.138793 & -0.011042 \\
\hline $\mathrm{H}$ & 0.171553 & 2.173927 & -0.017737 \\
\hline $\mathrm{C}$ & -1.515212 & 0.705736 & 0.014268 \\
\hline C & -1.515214 & -0.705731 & 0.014268 \\
\hline $\mathrm{H}$ & 0.171540 & -2.173933 & -0.017736 \\
\hline $\mathrm{H}$ & -2.396441 & -1.346912 & 0.006069 \\
\hline $\mathrm{H}$ & -2.396436 & 1.346921 & 0.006069 \\
\hline $\mathrm{C}$ & 2.163188 & -0.000001 & 0.017606 \\
\hline $\mathrm{H}$ & 2.580559 & -0.882746 & -0.487312 \\
\hline $\mathrm{H}$ & 2.581937 & -0.000146 & 1.038047 \\
\hline $\mathrm{H}$ & 2.580535 & 0.882901 & -0.487054 \\
\hline
\end{tabular}

$\begin{array}{lrr}\text { cyclopropenylcarbene+, } & \text { singlet } \\ \text { C } 0.000000 & 0.000000 & 0.386986 \\ \text { C } 0.000000 & 0.635725 & -1.004824 \\ \text { C } 0.000000 & -0.635725 & -1.004824 \\ \text { H } 0.000000 & 1.671886 & -1.317564 \\ \text { H } 0.000000 & -1.671886 & -1.317564 \\ \text { C } 0.000000 & 0.000000 & 1.613545 \\ \text { H } 0.000000 & 0.000000 & 2.689834\end{array}$

cyclopropenylcarbenet, triplet $\begin{array}{llll}\mathrm{H} & 1.738491 & -1.347969 & 0.000000\end{array}$

C $0.762172 \quad-0.878053 \quad 0.000000$ 


$\begin{array}{llrr}\text { C } & 0.000000 & 0.279476 & 0.000000 \\ \mathrm{C}-0.589792 & -0.979579 & 0.000000 \\ \mathrm{C}-0.067474 & 1.670052 & 0.000000 \\ \mathrm{H}-1.487304 & -1.586519 & 0.000000 \\ \mathrm{H}-0.880620 & 2.383115 & 0.000000\end{array}$

cyclopropenyl+CH2+
C 0.000000
0.000000
0.170281
C 0.000000
0.701836
$-1.010125$
C 0.000000
$-0.701836$
$-1.010125$
$\mathrm{H} 0.000000$
1.640171
$-1.572117$
$\mathrm{H} 0.000000$
$-1.640171$
$-1.572117$
C 0.000000
0.000000
1.643168
$\mathrm{H} 0.000000$
$-0.950878$
2. 192517
$\mathrm{H} 0.000000$
0.950878
2. 192517

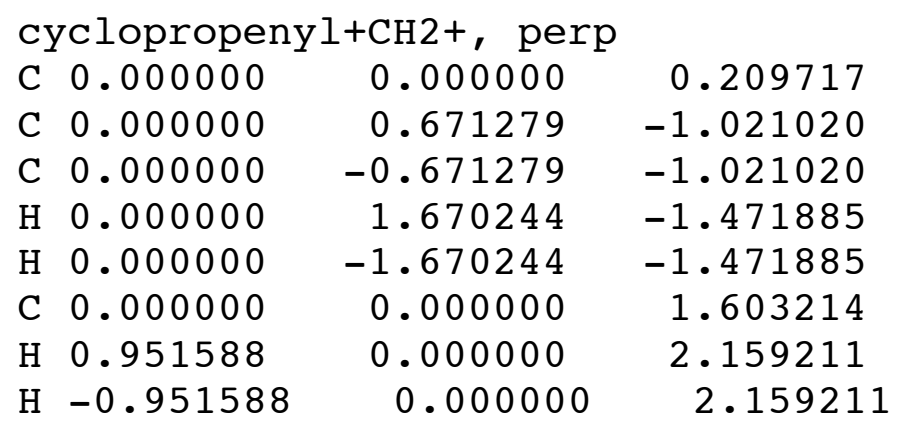

cyclopropenylCH3+

\begin{tabular}{|c|c|c|}
\hline C -0.123044 & -0.000001 & -0.012810 \\
\hline C 1.073435 & -0.681075 & 0.001589 \\
\hline 1.073434 & 0.681076 & 0.001587 \\
\hline 1.627260 & -1.612003 & 0.004078 \\
\hline 1.627260 & 1.612004 & 0.004073 \\
\hline-1.588362 & 0.000000 & -0.004837 \\
\hline-1.976 & 0.900321 & -0.482252 \\
\hline-1.913 & -0.000112 & 1.043382 \\
\hline-1.97 & -0.900211 & -0.482458 \\
\hline
\end{tabular}

cyclopropenylidene, singlet
C 0.000000
0.000000
0.938956
C 0.000000
0.664285
$-0.324393$
C 0.000000
$-0.664285$
$-0.324393$
$\mathrm{H} 0.000000$
$-1.596901$
$-0.870506$
$\mathrm{H} 0.000000$
1.596901
$-0.870506$

cyclopropenylidene, triplet
C 0.000000
0.000000
0.966529
C 0.000000
0.668641
$-0.327914$
C 0.000000
$-0.668641$
$-0.327914$ 


$\begin{array}{llrr}\mathrm{H} & 0.000000 & -1.562516 & -0.932103 \\ \mathrm{H} & 0.000000 & 1.562516 & -0.932103\end{array}$

\begin{tabular}{lrrr}
\multicolumn{4}{l}{ Cyclopropenium+ } \\
H 0.000000 & 0.000000 & 1.872746 \\
C & 0.000000 & 0.000000 & 0.789515 \\
C & 0.000000 & 0.683740 & -0.394757 \\
C & 0.000000 & -0.683740 & -0.394757 \\
H & 0.000000 & -1.621845 & -0.936374 \\
H & 0.000000 & 1.621845 & -0.936374
\end{tabular}

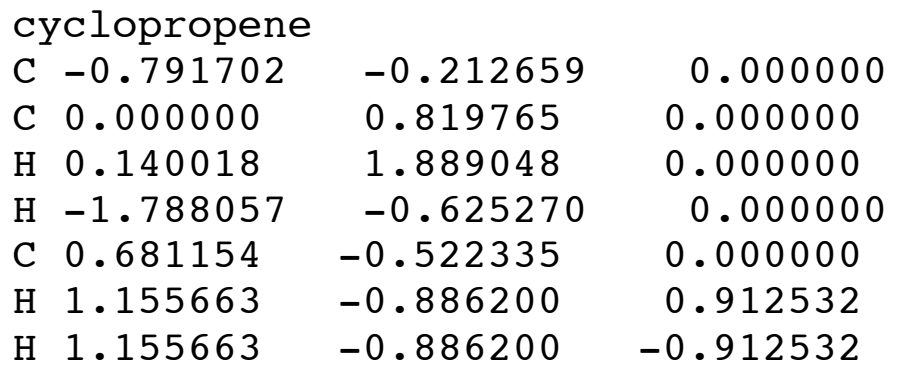

acyclic heteroatomcarbene (XY-C) sets

$\begin{array}{lcc}\text { aminocarbene, singlet } & \\ \mathrm{C} 0.062292 & 0.792190 & 0.000000 \\ \mathrm{~N} 0.062292 & -0.524892 & 0.000000 \\ \mathrm{H}-0.751328 & -1.142768 & 0.000000 \\ \mathrm{H} 0.949455 & -1.018070 & 0.000000 \\ \mathrm{H}-1.007919 & 1.081943 & 0.000000 \\ & & \\ \text { aminocarbene, } & \text { triplet } \\ \mathrm{H} 1.076072 & -0.831118 & -0.185777 \\ \mathrm{~N} 0.578175 & -0.000004 & 0.120534 \\ \mathrm{C}-0.772402 & 0.000010 & -0.173924 \\ \mathrm{H} 1.076081 & 0.831119 & -0.185735 \\ \mathrm{H}-1.564968 & -0.000035 & 0.571314 \\ & & \\ \text { aminocarbenium+, }\left(\mathrm{CH}_{2}=\mathrm{NH}_{2}{ }^{+}\right) \\ \mathrm{N} 0.000000 & 0.000000 & 0.602556 \\ \mathrm{H} 0.000000 & 0.869951 & 1.140851 \\ \mathrm{H} 0.000000 & -0.869951 & 1.140851 \\ \mathrm{C} 0.000000 & 0.000000 & -0.678939 \\ \mathrm{H} 0.000000 & 0.944175 & -1.212980 \\ \mathrm{H} 0.000000 & -0.944175 & -1.212980 \\ \text { aminomethane } & & \\ \mathrm{N}-0.051681 & -0.759433 & 0.000000 \\ \mathrm{H} 0.457708 & -1.100130 & 0.812232 \\ \mathrm{H} 0.457708 & -1.100130 & -0.812232\end{array}$




$\begin{array}{ccc}\mathrm{C}-0.051681 & 0.704304 & 0.000000 \\ \mathrm{H}-0.941900 & 1.176202 & 0.000000 \\ \mathrm{H}-0.592732 & 1.057132 & 0.880564 \\ \mathrm{H}-0.592732 & 1.057132 & -0.880564\end{array}$

diaminocarbene, singlet

c $0.000000-0.614141$

$\begin{array}{lll}\mathrm{N}-1.111427 & 0.141919 & 0.000067\end{array}$

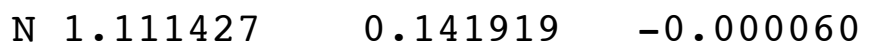

$\begin{array}{llll}\mathrm{H} & 2.009151 & -0.314562 & 0.000032\end{array}$

$\mathrm{H} 1.131514 \quad 1.163552 \quad 0.000114$

$\mathrm{H}-1.131514 \quad 1.163552 \quad-0.000063$

$\mathrm{H}-2.009151 \quad-0.314562 \quad 0.000000$

diaminocarbene, triplet

$\begin{array}{lll}\text { C }-0.001047 & 0.472099 & -0.165835\end{array}$

$\begin{array}{lll}\mathrm{N}-1.271454 & -0.024318 & 0.049577\end{array}$

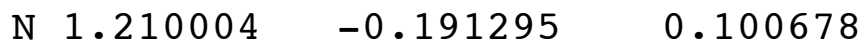

H $1.573688-0.708294 \quad-0.701576$

$\begin{array}{llll}\mathrm{H} & 1.928710 & 0.453522 & 0.416237\end{array}$

$\mathrm{H}-1.387975 \quad-0.504298 \quad 0.938687$

$\mathrm{H}-1.677989-0.564235 \quad-0.710131$

diaminocarbenium+

$\begin{array}{cccc}\mathrm{H} & 0.000000 & 0.000000 & 1.516239 \\ \mathrm{C} & 0.000000 & 0.000000 & 0.430729 \\ \mathrm{~N} & 0.000000 & 1.164447 & -0.175830 \\ \mathrm{~N} & 0.000000 & -1.164447 & -0.175830 \\ \mathrm{H} & 0.000000 & -1.263294 & -1.187448 \\ \mathrm{H} & 0.000000 & -2.021037 & 0.367954 \\ \mathrm{H} & 0.000000 & 2.021037 & 0.367954 \\ \mathrm{H} & 0.000000 & 1.263294 & -1.187448\end{array}$

diaminomethane

$\begin{array}{cccc}\mathrm{H} & 0.011391 & 1.095077 & 1.012849 \\ \mathrm{C} & 0.000748 & 0.545857 & 0.060350 \\ \mathrm{H}-0.040467 & 1.287494 & -0.742172 \\ \mathrm{~N}-1.161311 & -0.334191 & -0.108268 \\ \mathrm{~N} & 1.264683 & -0.157381 & -0.039646 \\ \mathrm{H} & 1.367279 & -0.791987 & 0.750611 \\ \mathrm{H} & 1.231164 & -0.745180 & -0.871632 \\ \mathrm{H} & -1.286551 & -0.900726 & 0.730077 \\ \mathrm{H} & -2.010907 & 0.221188 & -0.206434\end{array}$

hydroxycarbene, snglet, anti

$\begin{array}{lll}\mathrm{H} 1.240044 & 0.755196 & 0.000001\end{array}$

C $0.713167 \quad-0.225385 \quad 0.000000$

$\begin{array}{lll}0-0.552377 & 0.156300 & 0.000000\end{array}$ 


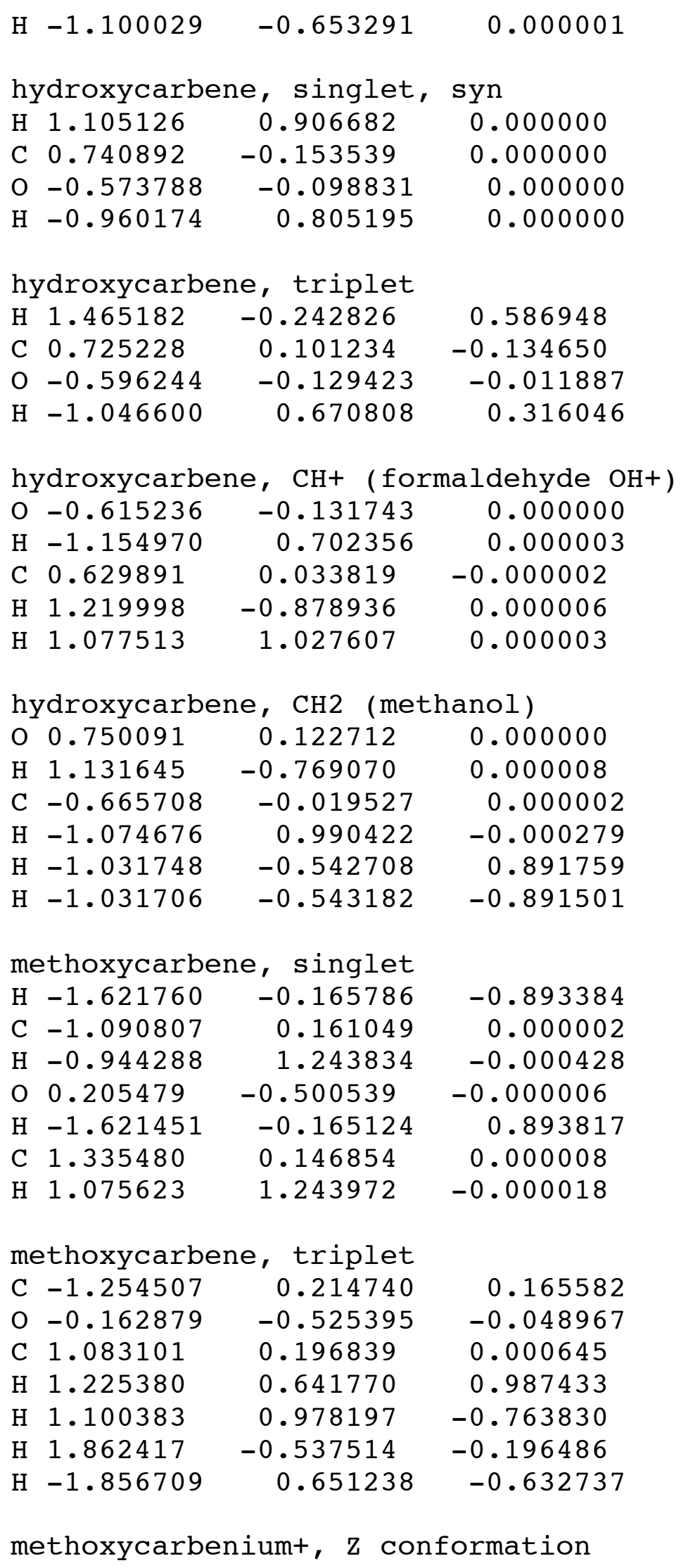




\begin{tabular}{|c|c|c|c|}
\hline $\mathrm{H}$ & 1.677743 & -0.232898 & 0.900347 \\
\hline $\mathrm{C}$ & 1.203663 & 0.149333 & 0.000000 \\
\hline & 1.093232 & 1.232555 & -0.000003 \\
\hline & -0.151406 & -0.479615 & 0.000000 \\
\hline & 1.677745 & -0.232903 & -0.900344 \\
\hline & -1.194861 & 0.204837 & 0.000000 \\
\hline & -1.160406 & 1.295729 & 0.000000 \\
\hline & -2.129883 & -0.350579 & 0.000001 \\
\hline \multicolumn{4}{|c|}{ dimethylether } \\
\hline $\mathrm{H}$ & 1.214027 & -0.840599 & -0.891754 \\
\hline C & 1.165542 & -0.199941 & 0.000000 \\
\hline $\mathrm{H}$ & 1.214057 & -0.840557 & 0.891783 \\
\hline O & 0.000000 & 0.600159 & 0.000000 \\
\hline $\mathrm{H}$ & 2.017696 & 0.480165 & -0.000030 \\
\hline $\mathrm{C}$ & -1.165542 & -0.199941 & 0.000000 \\
\hline & -1.214057 & -0.840556 & 0.891784 \\
\hline $\mathrm{H}$ & -1.214027 & -0.840600 & -0.891753 \\
\hline & -2.017696 & 0.480165 & -0.000032 \\
\hline
\end{tabular}

methoxymethylcarbene, singlet

$\begin{array}{cccc}\mathrm{H} & -2.265409 & -0.353090 & -0.892632 \\ \mathrm{C} & -1.792089 & 0.056510 & 0.000014 \\ \mathrm{H} & -1.812185 & 1.145348 & -0.000162 \\ \mathrm{O} & -0.413662 & -0.382779 & -0.000021 \\ \mathrm{H} & -2.265285 & -0.352798 & 0.892860 \\ \mathrm{C} & 0.481879 & 0.580862 & -0.000028 \\ \mathrm{C} & 1.826310 & -0.092492 & 0.000004 \\ \mathrm{H} & 2.378505 & 0.270290 & 0.873843 \\ \mathrm{H} & 2.378857 & 0.270746 & -0.873415 \\ \mathrm{H} & 1.798214 & -1.187550 & -0.000267\end{array}$

methoxymethylcarbene, triplet

$\begin{array}{lcr}\mathrm{H}-1.247369 & 1.401948 & -0.155341 \\ \mathrm{C}-1.610440 & 0.389754 & 0.045182 \\ \mathrm{H}-1.917663 & 0.309927 & 1.090277 \\ \mathrm{O}-0.593044 & -0.582932 & -0.256297 \\ \mathrm{H}-2.450058 & 0.160457 & -0.609728 \\ \mathrm{C} 0.550816 & -0.430715 & 0.437038 \\ \mathrm{C} 1.751978 & 0.276375 & -0.085082 \\ \mathrm{H} 2.557820 & 0.229342 & 0.649455 \\ \mathrm{H} 1.542263 & 1.334478 & -0.291947 \\ \mathrm{H} 2.105237 & -0.185181 & -1.015168 \\ & & \\ 1-\text { methoxyethyl+ } & \\ \mathrm{H}-2.303649 & -0.367908 & -0.899171 \\ \mathrm{C}-1.865519 & 0.057212 & 0.000000 \\ \mathrm{H}-1.880341 & 1.146230 & -0.000013\end{array}$




\begin{tabular}{llrr}
$\mathrm{O}$ & -0.457689 & -0.416808 & 0.000000 \\
$\mathrm{H}$ & -2.303642 & -0.367886 & 0.899185 \\
$\mathrm{C}$ & 0.508011 & 0.391101 & -0.000001 \\
$\mathrm{H}$ & 0.290558 & 1.464026 & 0.000001 \\
$\mathrm{C}$ & 1.880954 & -0.106905 & -0.000001 \\
$\mathrm{H}$ & 2.399898 & 0.303350 & -0.876092 \\
$\mathrm{H}$ & 1.918134 & -1.195109 & -0.000021 \\
$\mathrm{H}$ & 2.399880 & 0.303313 & 0.876120 \\
\multicolumn{3}{c}{$\mathrm{n}$} \\
$\mathrm{ethy}$ lmethylether \\
$\mathrm{H}-1.983147$ & 0.653601 & -0.891709 \\
$\mathrm{C}$ & -1.804474 & 0.036687 & 0.000000 \\
$\mathrm{H}$ & -1.983146 & 0.653601 & 0.891709 \\
$\mathrm{O}$ & -0.499323 & -0.508650 & 0.000000 \\
$\mathrm{H}$ & -2.499484 & -0.803576 & 0.000000 \\
$\mathrm{C}$ & 0.489902 & 0.508139 & 0.000000 \\
$\mathrm{H}$ & 0.371847 & 1.150689 & 0.886881 \\
$\mathrm{H}$ & 0.371847 & 1.150688 & -0.886881 \\
$\mathrm{C}$ & 1.843575 & -0.165641 & 0.000000 \\
$\mathrm{H}$ & 2.644418 & 0.578783 & 0.000000 \\
$\mathrm{H}$ & 1.949115 & -0.794848 & -0.885856 \\
$\mathrm{H}$ & 1.949115 & -0.794848 & 0.885856
\end{tabular}

dimethoxycarbene, singlet, $\mathrm{W}$ conformation
C 0.000000
0.360669
$-0.000002$
O 1.050414
$-0.455973$
$-0.000009$
C 2.312079
0.233178
0.000003
H 2.860584
$-0.071721$
$-0.891837$
H 2.860417
$-0.071408$
0.892055
O -1.050414
$-0.455974$
0.000009
C -2.312078
0.233178
$-0.000002$
$\mathrm{H}-2.860438$
$-0.071449$
$-0.892026$
$\mathrm{H}-2.142771$
1.309844
0.000147
$\mathrm{H}-2.860562$
$-0.071679$
0.891866

dimethoxycarbene, triplet
$\mathrm{H} 1.630072$
1.217069
0.932202
C 1.741426
0.745550
$-0.047244$
$\mathrm{H} 1.175193$
1.301438
$-0.799032$
O 1.288707
$-0.623681$
$-0.002890$
H 2.792512
0.705130
$-0.329058$
C 0.009502
$-0.770676$
0.388037
O -1.050776
$-0.469366$
C -1.924623
0.546935
$-0.405953$
$\mathrm{H}-1.378418$
1. 475850
0.119513
$\mathrm{H}-2.395597$
0.208506
0.303986
$\mathrm{H}-2.685037$
0.705529
1.044921
$-0.644114$ 


\begin{tabular}{lrr}
\multicolumn{4}{l}{ dimethoxycarbenium+, Z conformation } \\
C -0.032445 & 0.720838 & 0.000000 \\
O -0.744576 & -0.337063 & 0.000000 \\
C -2.222916 & -0.229684 & 0.000000 \\
H -2.515811 & 0.819417 & -0.000003 \\
H -2.550589 & -0.743249 & 0.899431 \\
H -2.550589 & -0.743255 & -0.899427 \\
O 1.235355 & 0.669629 & 0.000000 \\
C 1.913981 & -0.649937 & 0.000000 \\
H 1.621150 & -1.183213 & 0.901692 \\
H 2.967570 & -0.393881 & -0.000001 \\
H 1.621150 & -1.183214 & -0.901691 \\
H -0.470831 & 1.719565 & 0.000000
\end{tabular}

dimethoxymethane

$\begin{array}{ccrr}\mathrm{H} & 2.169498 & -0.446607 & 1.192188 \\ \mathrm{C} & 2.152221 & -0.256614 & 0.110151 \\ \mathrm{H} & 2.639830 & 0.708262 & -0.083841 \\ \mathrm{O} & 0.831814 & -0.284827 & -0.402936 \\ \mathrm{H} & 2.703767 & -1.044490 & -0.401732 \\ \mathrm{C} & 0.025229 & 0.706161 & 0.200563 \\ \mathrm{H} & 0.366583 & 1.711392 & -0.079849 \\ \mathrm{H} & 0.071571 & 0.593801 & 1.301430 \\ \mathrm{O} & -1.277013 & 0.589740 & -0.260514 \\ \mathrm{C} & -1.882664 & -0.638175 & 0.135107 \\ \mathrm{H} & -1.819510 & -0.769108 & 1.223490 \\ \mathrm{H} & -1.409903 & -1.490180 & -0.357796 \\ \mathrm{H} & -2.928968 & -0.570599 & -0.161213\end{array}$

fluorocarbene, singlet

$\begin{array}{cccc}\text { F } & 0.068512 & -0.591459 & 0.000000 \\ \mathrm{C} & 0.068512 & 0.727258 & 0.000000 \\ \mathrm{H}-1.027686 & 0.959582 & 0.000000\end{array}$

fluorocarbene, triplet

$\begin{array}{cccc}\text { F } & 0.057997 & -0.615966 & 0.000000 \\ \mathrm{C} & 0.057997 & 0.711544 & 0.000000 \\ \mathrm{H} & -0.869957 & 1.274428 & 0.000000\end{array}$

fluorocarbenium+
F $0.000000 \quad 0.000000$
0.642803
C $0.000000 \quad 0.000000$
$-0.599629$
$\mathrm{H} 0.000000$
0.972255
$-1.093724$
$\mathrm{H} 0.000000$
$-0.972255$
$-1.093724$

fluoromethane
F 0.000000
0.000000
0.754749
C 0.000000
0.000000
$-0.635465$ 


\begin{tabular}{|c|c|c|}
\hline $\mathrm{H} 0.000000$ & 1.031387 & -0.993319 \\
\hline $\mathrm{H}-0.893207$ & -0.515693 & -0.993319 \\
\hline $\mathrm{H} \quad 0.893207$ & -0.515693 & -0.993319 \\
\hline \multicolumn{3}{|c|}{ difluorocarbene, singlet } \\
\hline $\mathrm{F} 0.000000$ & 1.036211 & -0.201548 \\
\hline C 0.000000 & 0.000000 & 0.604644 \\
\hline F 0.000000 & -1.036211 & -0.201548 \\
\hline \multicolumn{3}{|c|}{ difluorocarbene, triplet } \\
\hline$F 0.000000$ & 1.148558 & -0.166287 \\
\hline C 0.000000 & 0.000000 & 0.498861 \\
\hline F 0.000000 & -1.148558 & -0.16628 \\
\hline \multicolumn{3}{|c|}{ difluorocarbenium+ } \\
\hline $\mathrm{H} 0.000000$ & 0.000000 & 1.508067 \\
\hline C 0.000000 & 0.000000 & 0.415503 \\
\hline F 0.000000 & 1.066609 & -0.222283 \\
\hline $\mathrm{F} 0.000000$ & -1.066609 & -0.222283 \\
\hline \multicolumn{3}{|c|}{ difluoromethane } \\
\hline Н 0.908454 & 0.000000 & 1.106712 \\
\hline C 0.000000 & 0.000000 & 0.502939 \\
\hline $\mathrm{F} 0.000000$ & 1.109672 & -0.290615 \\
\hline $\mathrm{H}-0.908454$ & 0.000000 & 1.106712 \\
\hline F 0.000000 & -1.109672 & -0.290615 \\
\hline \multicolumn{3}{|c|}{ silylcarbene, singlet } \\
\hline $\mathrm{H}-1.170529$ & -1.204592 & -0.527134 \\
\hline $\mathrm{Si}-0.505703$ & 0.011260 & -0.007137 \\
\hline C 1.358243 & -0.168369 & -0.040552 \\
\hline $\mathrm{H}-1.191378$ & 1.217986 & -0.549536 \\
\hline $\mathrm{H}-0.582404$ & 0.032688 & 1.486983 \\
\hline H 1.874695 & 0.806500 & -0.067083 \\
\hline \multicolumn{3}{|c|}{ silylcarbene, triplet } \\
\hline $\mathrm{H}-1.077696$ & 1.358443 & -0.000439 \\
\hline Si -0.507823 & -0.010146 & 0.000000 \\
\hline C 1.317672 & 0.109345 & 0.000002 \\
\hline $\mathrm{H}-0.991966$ & -0.731680 & 1.206248 \\
\hline $\mathrm{H}-0.991909$ & -0.732433 & -1.205821 \\
\hline $\mathrm{H} 2.265058$ & -0.408353 & 0.000001 \\
\hline \multicolumn{3}{|l|}{ methylsilyl+ } \\
\hline $\mathrm{H}-1.408616$ & 1.251228 & 0.017026 \\
\hline $\mathrm{Si}-0.640393$ & 0.000032 & -0.004755 \\
\hline $\mathrm{H} \quad 1.524051$ & -0.003132 & 1.039093 \\
\hline $\mathrm{H}-1.407677$ & -1.251749 & 0.016987 \\
\hline
\end{tabular}




\begin{tabular}{|c|c|c|}
\hline C 1.180442 & 0.000137 & -0.008004 \\
\hline H 1.587134 & -0.897244 & -0.481682 \\
\hline 1.587965 & 0.899626 & -0.476825 \\
\hline \multicolumn{3}{|l|}{ silylmethane } \\
\hline $\mathrm{H} \quad 1.158643$ & -1.077703 & -0.879842 \\
\hline Si 0.635703 & 0.000000 & 0.000000 \\
\hline H 1.158639 & -0.223115 & 1.373240 \\
\hline $\mathrm{H} 1.158640$ & 1.300819 & -0.493395 \\
\hline C -1.244482 & 0.000000 & 0.000000 \\
\hline $\mathrm{H}-1.636289$ & 0.790097 & 0.644756 \\
\hline $\mathrm{H}-1.636290$ & -0.953423 & 0.361867 \\
\hline $\mathrm{H}-1.636291$ & 0.163326 & -1.006622 \\
\hline \multicolumn{3}{|c|}{ disilylcarbene, singlet } \\
\hline Si 1.676741 & 0.088236 & -0.008601 \\
\hline C 0.000004 & -0.671104 & -0.086663 \\
\hline $\mathrm{Si}-1.676743$ & 0.088234 & -0.008601 \\
\hline $\mathrm{H} \quad 2.723404$ & -0.822281 & -0.531884 \\
\hline H 1.915426 & 1.448690 & -0.570076 \\
\hline $\mathrm{H} \quad 1.744478$ & 0.151565 & 1.482382 \\
\hline $\mathrm{H}-2.723456$ & -0.822363 & -0.531642 \\
\hline $\mathrm{H}-1.744413$ & 0.151855 & 1.482370 \\
\hline $\mathrm{H}-1.915437$ & 1.448575 & -0.570345 \\
\hline \multicolumn{3}{|c|}{ disilylcarbene, triplet } \\
\hline $\mathrm{Si}-1.831333$ & 0.000002 & 0.000001 \\
\hline C 0.000000 & -0.000017 & -0.000006 \\
\hline $\mathrm{Si} 1.831333$ & 0.000002 & 0.000001 \\
\hline $\mathrm{H}-2.344197$ & -0.304883 & 1.360695 \\
\hline $\mathrm{H}-2.344187$ & 1.330847 & -0.416300 \\
\hline H -2.344214 & -1.025941 & -0.944385 \\
\hline Н 2.344195 & -0.305370 & 1.360586 \\
\hline H 2.344215 & -1.025604 & -0.944751 \\
\hline H 2.344188 & 1.330996 & -0.415823 \\
\hline \multicolumn{2}{|c|}{$\mu$-hydridodisilylmethane+ } & $\left(C_{2 v}\right)$ \\
\hline $\mathrm{H}-0.000001$ & 1.765568 & -0.898291 \\
\hline C 0.000000 & 1.145421 & -0.000001 \\
\hline Si 1.249270 & -0.237187 & -0.000001 \\
\hline Si -1.249270 & -0.237187 & 0.000001 \\
\hline $\mathrm{H}-1.885770$ & -0.604579 & -1.270348 \\
\hline $\mathrm{H}-1.885768$ & -0.604578 & 1.270351 \\
\hline $\mathrm{H} \quad 0.000000$ & 1.765569 & 0.898289 \\
\hline $\mathrm{H} \quad 0.000002$ & -1.344119 & 0.000001 \\
\hline H $\quad 1.885770$ & -0.604576 & -1.270351 \\
\hline H 1.885772 & -0.604574 & 1.270349 \\
\hline
\end{tabular}




$\begin{array}{lcc}\text { silylmethylsilyliumt } & \left(\mathrm{H}_{3} \mathrm{Si}-\mathrm{CH}_{2}-\mathrm{SiH}_{2}{ }^{+}\right) \\ \mathrm{H} 2.159353 & -0.663982 & 1.252939 \\ \mathrm{Si} 1.571708 & -0.169711 & 0.000000 \\ \mathrm{C} 0.114592 & 0.859851 & 0.000001 \\ \mathrm{H} 2.159354 & -0.663976 & -1.252941 \\ \mathrm{Si}-1.534463 & -0.220710 & 0.000000 \\ \mathrm{H} 0.048125 & 1.478732 & -0.901156 \\ \mathrm{H} 0.048123 & 1.478732 & 0.901158 \\ \mathrm{H}-1.505679 & -1.051895 & 1.219959 \\ \mathrm{H}-2.612614 & 0.781117 & -0.000028 \\ \mathrm{H}-1.505656 & -1.051934 & -1.219932 \\ & & \\ \mathrm{disilylmethane} & & \\ \mathrm{H} 0.000000 & 1.485878 & 0.879004 \\ \mathrm{C} 0.000000 & 0.831113 & 0.000000 \\ \mathrm{H} 0.000000 & 1.485878 & -0.879004 \\ \mathrm{Si}-1.582276 & -0.183860 & 0.000000 \\ \mathrm{Si} 1.582276 & -0.183860 & 0.000000 \\ \mathrm{H} 1.636312 & -1.050568 & 1.206447 \\ \mathrm{H} 1.636313 & -1.050566 & -1.206448 \\ \mathrm{H} 2.780849 & 0.695955 & 0.000001 \\ \mathrm{H}-1.636313 & -1.050565 & 1.206448 \\ \mathrm{H}-2.780849 & 0.695955 & -0.000002 \\ \mathrm{H}-1.636311 & -1.050569 & -1.206446 \\ \mathrm{Silylethene} & & \\ \mathrm{H} 1.626487 & 1.366863 & -0.000308 \\ \mathrm{C} 1.741285 & 0.286016 & 0.000047 \\ \mathrm{H} 2.764359 & -0.082182 & 0.000826 \\ \mathrm{Si}-1.079564 & 0.077455 & 0.000067 \\ \mathrm{H}-1.057441 & 1.563029 & -0.000048 \\ \mathrm{H}-1.814872 & -0.399989 & -1.200744 \\ \mathrm{H}-1.813902 & -0.400407 & 1.201281 \\ \mathrm{C} 0.681306 & -0.539301 & -0.000382 \\ \mathrm{H} 0.873724 & -1.611971 & 0.000066 \\ & & \end{array}$

(silylmethyl)carbene, triplet

\begin{tabular}{|c|c|c|}
\hline $\mathrm{H}-0.815850$ & 1.365001 & 0.772224 \\
\hline-0.732794 & 0.631237 & -0.038202 \\
\hline-0.865188 & 1.191803 & -0.975791 \\
\hline Si 1.012027 & -0.115068 & 0.005923 \\
\hline 1.260885 & -0.738779 & 1.329414 \\
\hline 1.133675 & -1.154099 & -1.048742 \\
\hline 2.036503 & 0.936039 & -0.235884 \\
\hline-1.765776 & -0.406293 & 0.118704 \\
\hline-1.926976 & -1.338678 & -0.407158 \\
\hline
\end{tabular}

2-silylethyl+ (symmetrically silyl-bridged cation) 


$\begin{array}{lcc}\mathrm{H} 1.283884 & 1.242676 & -0.912884 \\ \mathrm{C} 1.136081 & 0.682468 & 0.006858 \\ \mathrm{H} 1.122999 & 1.247586 & 0.938204 \\ \mathrm{C} 1.136343 & -0.682333 & 0.006764 \\ \mathrm{Si}-1.034247 & -0.000030 & -0.005401 \\ \mathrm{H}-1.321015 & -1.237227 & -0.744044 \\ \mathrm{H}-1.327147 & -0.000815 & 1.431003 \\ \mathrm{H}-1.321411 & 1.237433 & -0.743421 \\ \mathrm{H} 1.123369 & -1.247589 & 0.938007 \\ \mathrm{H} 1.284231 & -1.242451 & -0.912977 \\ & & \\ \mathrm{Sily} & & \\ \mathrm{H} 0.647972 & 1.329132 & -0.875743 \\ \mathrm{C} 0.562740 & 0.675738 & -0.000001 \\ \mathrm{H} 0.647971 & 1.329132 & 0.875742 \\ \mathrm{Si}-1.144369 & -0.121714 & 0.000000 \\ \mathrm{H}-1.309079 & -0.978237 & -1.205556 \\ \mathrm{H}-1.309171 & -0.978021 & 1.205696 \\ \mathrm{H}-2.219132 & 0.906721 & -0.000132 \\ \mathrm{C} 1.700235 & -0.350984 & 0.000000 \\ \mathrm{H} 2.678761 & 0.137508 & 0.000007 \\ \mathrm{H} 1.653002 & -0.995378 & -0.882395 \\ \mathrm{p} \text { phosphinocarbene, singlet } & \\ \mathrm{H} 1.182818 & -1.149392 & 0.000138 \\ \mathrm{P} 0.423797 & 0.017392 & -0.000032 \\ \mathrm{H} 1.369941 & 1.077252 & 0.000174 \\ \mathrm{C}-1.187929 & -0.157964 & 0.000026 \\ \mathrm{H}-1.782135 & 0.759040 & 0.000007\end{array}$

phosphinocarbene, triplet
H 0.906967
1.047789
0.730730
P 0.511783
$-0.000015$
$-0.135444$
H 0.906998
$-1.047632$
0.730951
C -1.234059
0.000030
0.176628
$\mathrm{H}-2.086361$
$-0.000110$
$-0.489787$

phosphinocarbenium+

$\begin{array}{llcr}\mathrm{H} & 1.233881 & -1.188572 & 0.000023 \\ \mathrm{P} & 0.510119 & 0.000000 & -0.000015 \\ \mathrm{H} & 1.233881 & 1.188572 & 0.000028 \\ \mathrm{C} & -1.127876 & 0.000000 & 0.000021 \\ \mathrm{H} & -1.676147 & 0.938527 & 0.000028 \\ \mathrm{H}-1.676147 & -0.938527 & 0.000024\end{array}$

phosphinomethane
C -0.069213
1.185321
0.000000
$\mathrm{H}-0.607992$
1.540751
0.880674 


\begin{tabular}{|c|c|c|c|}
\hline $\mathrm{H}$ & -0.607992 & 1.540751 & -0.880674 \\
\hline $\mathrm{H}$ & 0.929050 & 1.624306 & 0.000000 \\
\hline & -0.069213 & -0.672138 & 0.000000 \\
\hline & 0.870206 & -0.867835 & -1.041984 \\
\hline & 0.870206 & -0.867835 & 1.041984 \\
\hline \multicolumn{2}{|c|}{ thiocarbene, } & \multicolumn{2}{|c|}{ singlet, anti } \\
\hline & 1.620043 & -0.774735 & 0.000010 \\
\hline & 1.151898 & 0.226239 & -0.000002 \\
\hline S & -0.471670 & -0.107486 & 0.000000 \\
\hline $\mathrm{H}$ & -0.984707 & 1.137075 & 0.000008 \\
\hline \multicolumn{2}{|c|}{ thiocarbene, } & \multicolumn{2}{|c|}{ singlet, syn } \\
\hline $\mathrm{H}$ & -1.602104 & 0.876956 & 0.000000 \\
\hline $\mathrm{C}$ & -1.158131 & -0.128687 & 0.000000 \\
\hline$S$ & 0.475736 & -0.082168 & 0.000000 \\
\hline $\mathrm{H}$ & 0.939121 & 1.209850 & 0.000000 \\
\hline \multicolumn{2}{|c|}{ thiocarbene, } & \multicolumn{2}{|l|}{ triplet } \\
\hline $\mathrm{H}$ & -1.952143 & -0.016014 & 0.610808 \\
\hline & -1.179107 & 0.041286 & -0.146667 \\
\hline S & 0.512679 & -0.090709 & 0.013461 \\
\hline $\mathrm{H}$ & 0.823929 & 1.219642 & 0.053827 \\
\hline \multicolumn{2}{|c|}{ thiocarbene } & \multicolumn{2}{|l|}{$\mathrm{CH}+$} \\
\hline$S$ & 0.548580 & -0.090533 & 0.000000 \\
\hline $\mathrm{H}$ & 0.846404 & 1.223378 & 0.000001 \\
\hline $\mathrm{C}$ & -1.065834 & 0.025095 & 0.000000 \\
\hline $\mathrm{H}$ & -1.635668 & -0.901304 & 0.000001 \\
\hline $\mathrm{H}$ & -1.593011 & 0.975882 & -0.000001 \\
\hline \multicolumn{2}{|c|}{ thiomethane } & \multicolumn{2}{|c|}{ (methanethiol) } \\
\hline S & 0.047943 & -0.664565 & 0.000000 \\
\hline $\mathrm{H}$ & -1.282097 & -0.825738 & 0.000000 \\
\hline $\mathrm{C}$ & 0.047943 & 1.149294 & 0.000000 \\
\hline $\mathrm{H}$ & 1.092126 & 1.461722 & 0.000000 \\
\hline $\mathrm{H}$ & -0.432384 & 1.550650 & 0.891949 \\
\hline $\mathrm{H}$ & -0.432384 & 1.550650 & -0.891949 \\
\hline
\end{tabular}

methylthiocarbene, singlet
S 0.214775
$-0.506590$
0.000000
C 1.283669
0.737760
$-0.000001$
H 2.277282
0.252894
0.000005
C -1.388021
0.325342
0.000000
$\mathrm{H}-1.950299$
0.037994
$-0.889864$
$\mathrm{H}-1.187073$
1. 397742
H -1.950199
0.038197
$-0.000131$
0.889993 
methylthiocarbene, triplet

\begin{tabular}{llrr} 
S & 0.179986 & -0.572563 & 0.016874 \\
$\mathrm{C}$ & 1.371444 & 0.619747 & \multicolumn{1}{c}{0.147563} \\
$\mathrm{H}$ & 1.953926 & 1.137012 & 0.607800 \\
$\mathrm{C}$ & -1.346715 & 0.418351 & 0.001990 \\
$\mathrm{H}$ & -2.180403 & -0.283798 & 0.056666 \\
$\mathrm{H}$ & -1.418907 & 0.990970 & -0.922183 \\
$\mathrm{H}$ & -1.382763 & 1.088238 & 0.861172
\end{tabular}

methanethiomethyl+

$\begin{array}{llcr}\text { S } & 0.137806 & -0.560202 & 0.000000 \\ \text { C } & -1.402576 & 0.379019 & 0.000000 \\ \text { H } & -1.963193 & 0.089760 & -0.891098 \\ \text { H } & -1.203183 & 1.450451 & -0.000019 \\ \text { H } & -1.963177 & 0.089788 & 0.891118 \\ \text { C } & 1.313280 & 0.543331 & 0.000000 \\ \text { H } & 1.120002 & 1.613855 & -0.000001 \\ \text { H } & 2.340437 & 0.185285 & 0.000001\end{array}$

methylthiomethane

$\begin{array}{llrr}\text { S } & 0.000000 & 0.663985 & 0.000000 \\ \mathrm{C} & -1.367122 & -0.513548 & 0.000000 \\ \mathrm{H} & -2.296668 & 0.058266 & -0.000001 \\ \mathrm{H} & -1.346723 & -1.144429 & 0.891644 \\ \mathrm{H} & -1.346723 & -1.144430 & -0.891643 \\ \mathrm{C} & 1.367122 & -0.513548 & 0.000000 \\ \mathrm{H} & 2.296668 & 0.058266 & -0.000003 \\ \mathrm{H} & 1.346721 & -1.144432 & -0.891642 \\ \mathrm{H} & 1.346724 & -1.144427 & 0.891645\end{array}$

chlorocarbene, singlet
C 0.045033
1.190167
0.000000
Cl $0.045033 \quad-0.504710$
0.000000
H $-1.035769 \quad 1.439066$
0.000000

chlorocarbene, triplet
C 0.036544
Cl 0.036544
1.157038
$\mathrm{H}-0.840523$
$-0.513669$
0.000000
0.000000
1.790143
0.000000

chlorocarbenium+
Cl 0.000000
0.000000
0.550610
C 0.000000
0.000000
$-1.036821$
$\mathrm{H} 0.000000$
0.949935
$-1.569720$
$\mathrm{H} 0.000000$
$-0.949935$
$-1.569720$
chloromethane
Cl $0.000000 \quad 0.000000 \quad 0.655761$ 


\begin{tabular}{|c|c|c|}
\hline C 0.000000 & 0.000000 & -1.121188 \\
\hline $\mathrm{H} 0.000000$ & 1.029068 & -1.473601 \\
\hline $\mathrm{H}-0.891199$ & -0.514534 & -1.473601 \\
\hline Н 0.891199 & -0.514534 & -1.473601 \\
\hline \multicolumn{3}{|c|}{ dichlorocarbene, singlet } \\
\hline $\mathrm{Cl} 0.000000$ & 1.405198 & -0.147622 \\
\hline C 0.000000 & 0.000000 & 0.836523 \\
\hline $\mathrm{Cl} 0.000000$ & -1.405198 & -0.147622 \\
\hline \multicolumn{3}{|c|}{ dichlorocarbene, triplet } \\
\hline $\mathrm{Cl} 0.000000$ & 1.506798 & -0.111246 \\
\hline C 0.000000 & 0.000000 & 0.630392 \\
\hline Cl 0.000000 & -1.506798 & -0.111246 \\
\hline \multicolumn{3}{|c|}{ dichlorocarbenium+ } \\
\hline $\mathrm{H} 0.000000$ & 0.000000 & 1.693371 \\
\hline C 0.000000 & 0.000000 & 0.601951 \\
\hline $\mathrm{Cl} 0.000000$ & 1.432493 & -0.156032 \\
\hline $\mathrm{Cl} 0.000000$ & -1.432493 & -0.156032 \\
\hline \multicolumn{3}{|c|}{ dichloromethane } \\
\hline $\mathrm{Cl} 0.000000$ & 1.473658 & -0.215165 \\
\hline C 0.000000 & 0.000000 & 0.760303 \\
\hline $\mathrm{H}-0.894257$ & 0.000000 & 1.376898 \\
\hline H 0.894257 & 0.000000 & 1.376898 \\
\hline $\mathrm{Cl} 0.000000$ & -1.473658 & -0.215165 \\
\hline \multicolumn{3}{|c|}{ "nitrosocarbene, singlet", (hydrogen cyanide } \\
\hline C 0.000000 & 0.000000 & -1.198167 \\
\hline $\mathrm{N} 0.000000$ & 0.000000 & -0.013964 \\
\hline 00.000000 & 0.000000 & 1.193461 \\
\hline $\mathrm{H} 0.000000$ & 0.000000 & -2.260936 \\
\hline \multicolumn{3}{|c|}{ nitrosocarbene, triplet (anti) } \\
\hline C 1.160552 & 0.036162 & 0.000000 \\
\hline H 1.627187 & -0.949427 & 0.000000 \\
\hline $\mathrm{N} 0.000000$ & 0.393593 & 0.000000 \\
\hline $0-1.073812$ & -0.252836 & 0.000000 \\
\hline \multicolumn{3}{|c|}{ nitrosomethyl+ $\left(\mathrm{H}_{2} \mathrm{C}=\mathrm{N}=\mathrm{O}\right)^{+}$} \\
\hline $\mathrm{H}-0.000025$ & -1.675339 & 0.973056 \\
\hline C -0.000026 & -1.176265 & 0.000000 \\
\hline $\mathrm{N} 0.000000$ & 0.077537 & 0.000000 \\
\hline $\mathrm{H}-0.000025$ & -1.675339 & -0.973056 \\
\hline 00.000025 & 1.233189 & 0.000000 \\
\hline
\end{tabular}




\begin{tabular}{|c|c|c|}
\hline $\mathrm{H}-1.636134$ & -0.193909 & -0.884678 \\
\hline$C-1.093627$ & 0.150355 & -0.000001 \\
\hline$H-0.965252$ & 1.234918 & -0.000025 \\
\hline $\mathrm{N} 0.214909$ & -0.539560 & 0.000000 \\
\hline $\mathrm{H}-1.636109$ & -0.193869 & 0.884708 \\
\hline O 1.161862 & 0.253456 & 0.000000 \\
\hline \multicolumn{3}{|c|}{ iminocarbene, singlet } \\
\hline $\mathrm{H} 0.000000$ & 0.000000 & 1.438998 \\
\hline $\mathrm{N} 0.000000$ & 0.000000 & 0.436514 \\
\hline C 0.000000 & 0.000000 & -0.749099 \\
\hline \multicolumn{3}{|c|}{ iminocarbene, triplet } \\
\hline C 0.067853 & 0.731546 & 0.000000 \\
\hline $\mathrm{N} 0.067853$ & -0.496928 & 0.000000 \\
\hline$C-0.882095$ & -0.910780 & 0.000000 \\
\hline \multicolumn{3}{|c|}{ iminocarbene, $\mathrm{CH}+$} \\
\hline $\mathrm{H} 0.000000$ & 0.000000 & 1.561926 \\
\hline $\mathrm{N} 0.000000$ & 0.000000 & 0.541752 \\
\hline C 0.000000 & 0.000000 & -0.610251 \\
\hline $\mathrm{H} \quad 0.000000$ & 0.000000 & -1.692687 \\
\hline \multicolumn{3}{|l|}{ formaldimine } \\
\hline C 0.056194 & 0.589905 & 0.000000 \\
\hline $\mathrm{H}-0.835006$ & 1.225617 & 0.000000 \\
\hline $\mathrm{H} 1.014426$ & 1.107643 & 0.000000 \\
\hline $\mathrm{N} 0.056194$ & -0.690739 & 0.000000 \\
\hline $\mathrm{H}-0.909939$ & -1.037522 & 0.000000 \\
\hline \multicolumn{3}{|c|}{ carbon monoxide, singlet } \\
\hline 00.000000 & 0.000000 & 0.492953 \\
\hline C 0.000000 & 0.000000 & -0.657271 \\
\hline \multicolumn{3}{|c|}{ carbon monoxide, triplet } \\
\hline 00.000000 & 0.000000 & 0.504219 \\
\hline C 0.000000 & 0.000000 & -0.672292 \\
\hline \multicolumn{3}{|c|}{ formylt (carbon monoxide, } \\
\hline 00.000000 & 0.000000 & 0.600565 \\
\hline C 0.000000 & 0.000000 & -0.529977 \\
\hline $\mathrm{H} 0.000000$ & 0.000000 & -1.624660 \\
\hline \multicolumn{3}{|l|}{ formaldehyde } \\
\hline 00.000000 & 0.000000 & 0.683479 \\
\hline C 0.000000 & 0.000000 & -0.536364 \\
\hline $\mathrm{H} 0.000000$ & 0.934472 & -1.124826 \\
\hline 0.000000 & -0.934472 & -1.124826 \\
\hline
\end{tabular}




\begin{tabular}{|c|c|c|c|}
\hline \multicolumn{4}{|c|}{ 2,2-diaminovinylt, singlet } \\
\hline $\mathrm{N}$ & -1.112067 & -0.671847 & -0.009730 \\
\hline $\mathrm{C}$ & -0.004022 & 0.063501 & 0.011955 \\
\hline $\mathrm{N}$ & 1.206526 & -0.484487 & 0.031598 \\
\hline 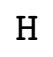 & -1.100857 & -1.637951 & -0.328188 \\
\hline 11 & -2.023579 & -0.261967 & 0.169050 \\
\hline $\mathrm{H}$ & 1.359770 & -1.441787 & 0.336484 \\
\hline $\mathrm{H}$ & 2.018609 & 0.070095 & -0.225326 \\
\hline 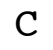 & -0.044820 & 1.496347 & -0.136912 \\
\hline $\mathrm{H}$ & -0.622103 & 2.006862 & 0.644648 \\
\hline & 2-diamino & nyl cation, & singlet ts \\
\hline $\mathrm{N}$ & 0.000000 & 1.172082 & -0.604429 \\
\hline 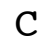 & 0.000000 & 0.000000 & 0.087590 \\
\hline C & 0.000000 & 0.000000 & 1.415472 \\
\hline $\mathrm{N}$ & 0.000000 & -1.172082 & -0.604429 \\
\hline $\mathrm{H}$ & 0.670693 & 1.265276 & -1.363092 \\
\hline $\mathrm{H}$ & -0.316407 & 2.028815 & -0.161952 \\
\hline $\mathrm{H}$ & -0.670693 & -1.265276 & -1.363092 \\
\hline & 0.316407 & -2.028815 & -0.161952 \\
\hline & 0.000000 & 0.000000 & 2.493725 \\
\hline
\end{tabular}

2,2-diaminovinyl+, triplet

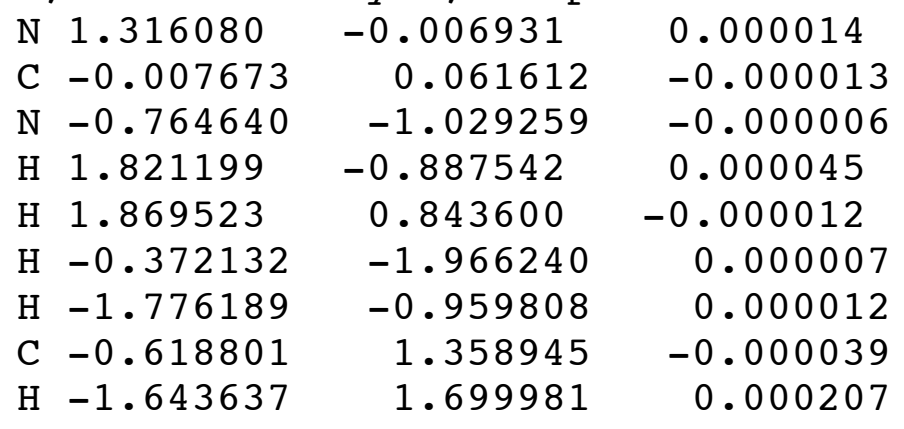

diaminomethyl-CH2 dication

$\begin{array}{cccc}\mathrm{N} & 1.181893 & 0.633591 & 0.000004 \\ \mathrm{C} & 0.000000 & 0.030597 & -0.000001 \\ \mathrm{~N} & -1.181891 & 0.633594 & -0.000004 \\ \mathrm{H} & 1.277451 & 1.657861 & 0.000015 \\ \mathrm{H} & 2.068520 & 0.119013 & -0.000023 \\ \mathrm{H} & -1.277446 & 1.657864 & -0.000008 \\ \mathrm{H} & -2.068520 & 0.119018 & 0.000020 \\ \mathrm{C} & -0.000002 & -1.433211 & 0.000000 \\ \mathrm{H} & 0.934982 & -2.004188 & -0.000003 \\ \mathrm{H} & -0.934987 & -2.004185 & 0.000006\end{array}$

1,1-diaminoethyl cation

$\mathrm{N} 0.705706 \quad-1.149481 \quad 0.000003$ 


$\begin{array}{llrr}\mathrm{C} & 0.057201 & 0.000000 & -0.015659 \\ \mathrm{~N} & 0.705666 & 1.149502 & 0.000005 \\ \mathrm{H} & 1.719023 & -1.213445 & 0.023439 \\ \mathrm{H} & 0.195808 & -2.025462 & -0.012296 \\ \mathrm{H} & 1.718980 & 1.213504 & 0.023445 \\ \mathrm{H} & 0.195738 & 2.025465 & -0.012306 \\ \mathrm{C} & -1.435616 & -0.000022 & 0.003578 \\ \mathrm{H} & -1.829573 & 0.885642 & -0.496125 \\ \mathrm{H} & -1.779545 & 0.000063 & 1.042247 \\ \mathrm{H} & -1.829545 & -0.885782 & -0.495974\end{array}$

\section{cyclic heteroatom carbene sets}

NOTE: where cis/trans isomerism exists, only the more stable of the two isomers is listed here.

\begin{tabular}{|c|c|c|c|}
\hline \multicolumn{4}{|c|}{ imidazol-2-ylidine, singlet } \\
\hline $\mathrm{H}$ & 0.000000 & 1.388832 & -1.751294 \\
\hline $\mathrm{C}$ & 0.000000 & 0.681319 & -0.935968 \\
\hline $\mathrm{C}$ & 0.000000 & -0.681319 & -0.935968 \\
\hline $\mathrm{N}$ & 0.000000 & 1.045771 & 0.398585 \\
\hline $\mathrm{H}$ & 0.000000 & -1.388832 & -1.751294 \\
\hline $\mathrm{N}$ & 0.000000 & -1.045771 & 0.398585 \\
\hline $\mathrm{H}$ & 0.000000 & 2.003972 & 0.720890 \\
\hline $\mathrm{H}$ & 0.000000 & -2.003972 & 0.720890 \\
\hline & 0.000000 & 0.000000 & 1.285373 \\
\hline \multicolumn{4}{|c|}{ imidazol-2-ylidene, triplet } \\
\hline $\mathrm{H}$ & 1.350537 & 1.749745 & -0.061926 \\
\hline $\mathrm{C}$ & 0.674185 & 0.907543 & -0.000895 \\
\hline $\mathrm{C}$ & -0.673803 & 0.907764 & -0.000956 \\
\hline $\mathrm{H}$ & -1.349839 & 1.750225 & -0.061932 \\
\hline $\mathrm{C}$ & -0.000200 & -1.196125 & -0.132075 \\
\hline $\mathrm{N}$ & 1.178448 & -0.422322 & -0.035947 \\
\hline $\mathrm{N}$ & -1.178662 & -0.421975 & -0.035941 \\
\hline $\mathrm{H}$ & 1.834407 & -0.652806 & 0.715267 \\
\hline$\Pi$ & -1.834699 & -0.652175 & 0.715356 \\
\hline \multicolumn{4}{|c|}{ imidazol-2-ylidene-2-CH+ } \\
\hline $\mathrm{H}$ & 0.000000 & 1.393514 & -1.788673 \\
\hline $\mathrm{C}$ & 0.000000 & 0.684743 & -0.974394 \\
\hline C & 0.000000 & -0.684743 & -0.974394 \\
\hline $\mathrm{N}$ & 0.000000 & 1.072375 & 0.344390 \\
\hline $\mathrm{H}$ & 0.000000 & -1.393514 & -1.788673 \\
\hline $\mathrm{N}$ & 0.000000 & -1.072375 & 0.344390 \\
\hline $\mathrm{H}$ & 0.000000 & 2.035160 & 0.676011 \\
\hline
\end{tabular}




$\begin{array}{rrrr}\mathrm{H} & 0.000000 & -2.035160 & 0.676011 \\ \mathrm{C} & 0.000000 & 0.000000 & 1.145212 \\ \mathrm{H} & 0.000000 & 0.000000 & 2.225317\end{array}$

$\begin{array}{lcr}2,2 \text {-dihydroimidazole, trans } \\ \text { H } 1.420422 & 1.783037 & 0.074281 \\ \text { C } 0.793727 & 0.902001 & 0.018585 \\ \text { C } 1.153271 & -0.388521 & 0.093841 \\ \text { H } 2.142558 & -0.814456 & 0.188723 \\ \text { C }-1.105911 & -0.300383 & 0.180529 \\ \text { H }-1.325486 & -0.294936 & 1.259659 \\ \text { H }-1.999843 & -0.595302 & -0.372990 \\ \mathrm{~N}-0.004643 & -1.240514 & -0.047944 \\ \mathrm{H}-0.050474 & -1.577463 & -1.013929 \\ \mathrm{~N}-0.594534 & 1.016555 & -0.256275 \\ \mathrm{H}-1.039454 & 1.788256 & 0.236058\end{array}$

\begin{tabular}{|c|c|c|c|}
\hline \multicolumn{4}{|c|}{ 4,5-dihydroimidazol-2-ylidene, singlet } \\
\hline $\mathrm{H}$ & 1.295023 & 1.579874 & -0.566742 \\
\hline $\mathrm{C}$ & 0.754707 & 0.922945 & 0.118831 \\
\hline $\mathrm{N}$ & 1.056400 & -0.495409 & -0.122095 \\
\hline $\mathrm{C}$ & -0.754708 & 0.922944 & -0.118830 \\
\hline $\mathrm{H}$ & 0.997271 & 1.207810 & 1.150693 \\
\hline $\mathrm{N}$ & -1.056399 & -0.495410 & 0.122095 \\
\hline $\mathrm{H}$ & -0.997273 & 1.207809 & -1.150692 \\
\hline $\mathrm{H}$ & -1.295025 & 1.579872 & 0.566742 \\
\hline $\mathrm{C}$ & 0.000001 & -1.334849 & 0.000000 \\
\hline $\mathrm{H}$ & 1.995972 & -0.852935 & -0.024466 \\
\hline $\mathrm{H}$ & -1.995971 & -0.852938 & 0.024467 \\
\hline \multicolumn{4}{|c|}{ 4,5-dihydroimidazol-2-ylidene, triplet } \\
\hline $\mathrm{H}$ & -1.388611 & 1.576188 & 0.295161 \\
\hline $\mathrm{C}$ & -0.767753 & 0.838703 & -0.218503 \\
\hline $\mathrm{C}$ & 0.718056 & 0.905346 & 0.172461 \\
\hline $\mathrm{H}$ & -0.880139 & 0.965216 & -1.297879 \\
\hline $\mathrm{N}$ & 1.186040 & -0.460058 & -0.155024 \\
\hline $\mathrm{H}$ & 0.817966 & 1.124759 & 1.244774 \\
\hline $\mathrm{H}$ & 1.275073 & 1.657977 & -0.392256 \\
\hline $\mathrm{C}$ & -0.001287 & -1.237285 & -0.164925 \\
\hline $\mathrm{H}$ & 1.899092 & -0.797121 & 0.490756 \\
\hline $\mathrm{N}$ & -1.185816 & -0.537755 & 0.126559 \\
\hline $\mathrm{H}$ & -1.419053 & -0.582911 & 1.124495 \\
\hline \multicolumn{4}{|c|}{$4,5-d i h y d r o i m i d a z o l-2-\mathrm{CH}+$} \\
\hline $\mathrm{H}$ & 1.110818 & 1.037248 & -1.332652 \\
\hline $\mathrm{C}$ & 0.114305 & 0.764279 & -0.977915 \\
\hline & 0.000000 & 1.092050 & 0.457619 \\
\hline
\end{tabular}




$\begin{array}{cccc}\mathrm{C} & -0.114305 & -0.764279 & -0.977915 \\ \mathrm{H} & -0.639601 & 1.302442 & -1.552476 \\ \mathrm{~N} & 0.000000 & -1.092050 & 0.457619 \\ \mathrm{H} & 0.639601 & -1.302442 & -1.552476 \\ \mathrm{H}-1.110818 & -1.037248 & -1.332652 \\ \mathrm{H} & 0.071613 & 2.029066 & 0.841298 \\ \mathrm{H} & -0.071613 & -2.029066 & 0.841298 \\ \mathrm{C} & 0.000000 & 0.000000 & 1.190223 \\ \mathrm{H} & 0.000000 & 0.000000 & 2.274632\end{array}$

\begin{tabular}{|c|c|c|c|}
\hline \multicolumn{4}{|c|}{$2,2,4,5$-tetrahydroimidazole cis } \\
\hline $\mathrm{H}$ & 0.940322 & 1.493238 & 1.214162 \\
\hline C & 0.081495 & 0.973160 & 0.781763 \\
\hline $\mathrm{N}$ & 0.081495 & -0.443505 & 1.189096 \\
\hline C & 0.081495 & 0.973160 & -0.781763 \\
\hline $\mathrm{H}$ & -0.824522 & 1.453598 & 1.162483 \\
\hline $\mathrm{N}$ & 0.081495 & -0.443505 & -1.189096 \\
\hline & 0.940322 & 1.493238 & -1.214162 \\
\hline & -0.824522 & 1.453598 & -1.162483 \\
\hline $\mathrm{H}$ & 1.052386 & -0.741083 & -1.296621 \\
\hline & -0.450674 & -1.116554 & 0.000000 \\
\hline & -0.211602 & -2.182554 & 0.000000 \\
\hline & -1.539598 & -0.998478 & 0.000000 \\
\hline & 1.052386 & -0.741083 & 1.296621 \\
\hline
\end{tabular}

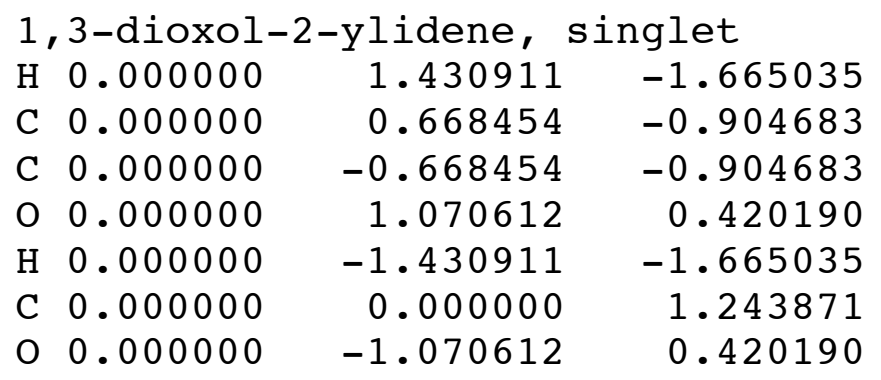

1,3-dioxol-2-ylidene, triplet
O 1.082269
$-0.429043$
0.002998
C 0.007142
$-1.230064$
0.044440
O -1.077129
$-0.441144$
0.006017
C -0.765327
0.930091
$-0.119408$
C 0.754856
0.939217
$-0.116348$
$\mathrm{H}-1.368035$
1.557031
0.532734
H 1.346884
1.568999
0.543046

\begin{tabular}{|c|c|c|}
\hline \multicolumn{3}{|c|}{1,3 -dioxole-2-CH+ } \\
\hline $\mathrm{H} 0.000000$ & 1.449351 & -1.701384 \\
\hline 0.000000 & 0.673562 & -0.950820 \\
\hline 0.000000 & -0.673562 & -0.950820 \\
\hline 0.000000 & 1.077635 & 0.378419 \\
\hline
\end{tabular}




\begin{tabular}{|c|c|c|c|}
\hline $\mathrm{H}$ & 0.000000 & -1.449351 & -1.701384 \\
\hline $\mathrm{O}$ & 0.000000 & -1.077635 & 0.378419 \\
\hline $\mathrm{C}$ & 0.000000 & 0.000000 & 1.096359 \\
\hline $\mathrm{H}$ & 0.000000 & 0.000000 & 2.179746 \\
\hline \multicolumn{4}{|c|}{1,3 -dioxole-2-CH2 } \\
\hline $\mathrm{H}$ & -1.767510 & 1.394782 & 0.099947 \\
\hline $\mathrm{C}$ & -0.973276 & 0.667673 & 0.043622 \\
\hline $\mathrm{C}$ & -0.973279 & -0.667670 & 0.043610 \\
\hline 0 & 0.319185 & 1.149243 & -0.139875 \\
\hline $\mathrm{H}$ & -1.767511 & -1.394779 & 0.099981 \\
\hline 0 & 0.319181 & -1.149245 & -0.139873 \\
\hline $\mathrm{C}$ & 1.119650 & -0.000002 & 0.136406 \\
\hline & 1.391490 & 0.000007 & 1.204109 \\
\hline & 1.998035 & -0.000008 & -0.507878 \\
\hline
\end{tabular}

$\begin{array}{lcc}\text { 4,5-dihydro-1,3-dioxole-2-ylidene, singlet } \\ \text { H }-0.893673 & 1.206100 & -1.340295 \\ \text { C } 0.000000 & 0.757126 & -0.906942 \\ \text { H } 0.893022 & 1.206166 & -1.341539 \\ \text { C } 0.000000 & -0.757126 & -0.906942 \\ \text { O }-0.000980 & -1.080943 & 0.528840 \\ \text { H } 0.893673 & -1.206100 & -1.340295 \\ \text { C } 0.000000 & 0.000000 & 1.297589 \\ \text { O } 0.000980 & 1.080943 & 0.528840 \\ \text { H }-0.893022 & -1.206166 & -1.341539\end{array}$

4,5-dihydro-1,3-dioxol-2-ylidene, triplet

$\mathrm{H}-0.842856 \quad 0.980189 \quad-1.286563$

C $-0.732949 \quad 0.843819 \quad-0.205459$

$\mathrm{H}-1.360556 \quad 1.551234 \quad 0.336736$

$\begin{array}{lll}\text { C } 0.733016 & 0.843410 & 0.206585\end{array}$

O $1.149253-0.488895 \quad-0.168533$

$\mathrm{H} 0.843161 \quad 0.976666 \quad 1.288060$

C $0.000657 \quad-1.226093 \quad 0.002453$

$\begin{array}{lll}0-1.149836 & -0.489485 & 0.165308\end{array}$

$\mathrm{H} 1.360572 \quad 1.552129 \quad-0.333909$

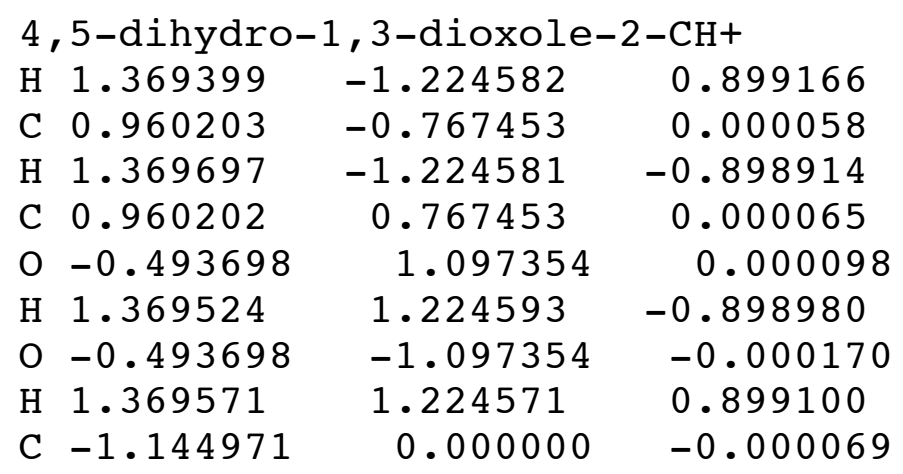




$$
\begin{aligned}
& \mathrm{H}-2.231624 \quad 0.000000 \quad-0.000124 \\
& 2,2,4,5 \text {-tetrahydro-1,3-dioxole } \\
& \mathrm{H}-0.106698 \quad 1.391528 \quad-1.659209 \\
& \text { C } 0.319000 \quad 0.686838 \quad-0.942815 \\
& \mathrm{H} 1.405324 \quad 0.617430 \quad-1.086649 \\
& \text { C }-0.319000 \quad-0.686838 \quad-0.942815 \\
& \begin{array}{llll}
0 & 0.000000 & 1.150418 & 0.370166
\end{array} \\
& \mathrm{H} 0.106698 \quad-1.391528-1.659209 \\
& \mathrm{H}-1.405324-0.617430 \quad-1.086649 \\
& \begin{array}{llll}
0 & 0.000000 & -1.150418 & 0.370166
\end{array} \\
& \text { C } 0.000000 \quad 0.000000 \quad 1.203980 \\
& \mathrm{H}-0.899646 \quad 0.044858 \quad 1.829485 \\
& \mathrm{H} \quad 0.899646 \quad-0.044858 \quad 1.829485
\end{aligned}
$$
$\mathrm{H}-1.957559$
1.026743
$-0.000005$
C -0.970264
0.593597
0.000007
C 0.276198
1.110141
0.000008
O -0.872662
$-0.776034$
0.000006
$\mathrm{H} 0.634033$
2.127483
$-0.000010$
N 1.103264
$-0.007713$
0.000004
C 0.438590
$-1.189622$
$-0.000028$
H 2.114827
0.023343
0.000019

oxazole-2-ylidene, triplet
H 1.247547
1.473720
0.791324
C 0.761948
0.946373
$-0.033055$
C -0.752526
0.942550
$-0.179258$
O 1.124929
$-0.418235$
$-0.075139$
$\mathrm{H}-1.399016$
1.697094
0.256199
C 0.041835
$-1.244384$
0.053404
$\mathrm{N}-1.027721 \quad-0.413212$
0.056654
$\mathrm{H}-1.961458$
$-0.799680$
0.110465

oxazole-2-CH+
$\mathrm{H} 2.150798$
$-0.646950$
0.000050
C 1.148823
$-0.246234$
0.000012
C 0.618023
1.003643
0.000008
O 0.118802
$-1.154157$
$-0.000037$
$\mathrm{H} 1.077585$
1.980810
0.000004
$\mathrm{N}-0.753508$
0.813238
0.000007
$\mathrm{H}-1.460913$
1.549589
0.000021
C -1.012829
$-0.482787$
0.000001
$\mathrm{H}-1.967430$
$-0.990595$
0.000045
oxazole-2-CH2
$\mathrm{H}-2.000543 \quad-1.043605 \quad 0.056673$ 


$\begin{array}{cccc}\mathrm{C} & -1.082295 & -0.475090 & 0.009408 \\ \mathrm{C} & -0.853920 & 0.842998 & 0.088090 \\ \mathrm{H} & -1.560633 & 1.651563 & 0.205594 \\ \mathrm{~N} & 0.562384 & 1.091750 & -0.057661 \\ \mathrm{H} & 0.754459 & 1.383350 & -1.019912 \\ \mathrm{O} & 0.075403 & -1.204260 & -0.174563 \\ \mathrm{C} & 1.099859 & -0.235289 & 0.184477 \\ \mathrm{H} & 2.000225 & -0.443206 & -0.393339 \\ \mathrm{H} & 1.284720 & -0.351984 & 1.259268\end{array}$

\begin{tabular}{|c|c|c|c|}
\hline \multicolumn{4}{|c|}{4,5 -dihydrooxazol-2-ylidene, singlet } \\
\hline $\mathrm{H}$ & 0.611696 & 1.586488 & -1.042163 \\
\hline $\mathrm{C}$ & 0.416068 & 1.124669 & -0.068952 \\
\hline $\mathrm{N}$ & 1.137564 & -0.146402 & 0.064207 \\
\hline C & -1.010933 & 0.607210 & 0.068938 \\
\hline $\mathrm{H}$ & 0.691806 & 1.827969 & 0.720213 \\
\hline $\mathrm{H}$ & -1.689086 & 0.955198 & -0.710136 \\
\hline $\mathrm{H}$ & -1.441392 & 0.812735 & 1.051695 \\
\hline O & -0.886412 & -0.849087 & -0.061746 \\
\hline C & 0.396307 & -1.258773 & -0.004108 \\
\hline $\mathrm{H}$ & 2.146673 & -0.203514 & 0.049647 \\
\hline
\end{tabular}

$\begin{array}{lcc}\text { 4,5-dihydrooxazol-2-ylidene, triplet } \\ \text { H }-1.193722 & 1.709357 & 0.298128 \\ \text { C }-0.656635 & 0.915734 & -0.225742 \\ \text { N }-1.212152 & -0.413244 & 0.109652 \\ \text { C } 0.819758 & 0.795956 & 0.176191 \\ \text { H }-0.752204 & 1.064959 & -1.303502 \\ \text { H } 0.970260 & 1.017390 & 1.239165 \\ \text { H } 1.501528 & 1.400868 & -0.424537 \\ \text { O } 1.132075 & -0.601504 & -0.053720 \\ \text { C }-0.099920 & -1.219166 & -0.158189 \\ \text { H }-1.476622 & -0.442987 & 1.099381\end{array}$

\begin{tabular}{|c|c|c|c|}
\hline \multicolumn{4}{|c|}{4,5 -dihydrooxazole-2-CH+ } \\
\hline $\mathrm{H}$ & 1.209213 & 1.426660 & 0.893415 \\
\hline $\mathrm{C}$ & 0.828678 & 0.929252 & 0.000072 \\
\hline $\mathrm{N}$ & -0.646797 & 0.963190 & 0.000065 \\
\hline $\mathrm{C}$ & 1.080016 & -0.592685 & 0.000015 \\
\hline $\mathrm{H}$ & 1.209217 & 1.426731 & -0.893230 \\
\hline $\mathrm{H}$ & 1.573916 & -0.963685 & 0.896207 \\
\hline $\mathrm{H}$ & 1.574051 & -0.963598 & -0.896138 \\
\hline 0 & -0.277112 & -1.198138 & -0.000113 \\
\hline $\mathrm{H}$ & -1.210233 & 1.811594 & 0.000118 \\
\hline $\mathrm{C}$ & -1.143793 & -0.238484 & -0.000055 \\
\hline $\mathrm{H}$ & -2.201095 & -0.483423 & -0.000109 \\
\hline
\end{tabular}

$2,2,4,5$-tetrahydrooxazole 


$\begin{array}{llcc}\mathrm{C} & -1.095823 & -0.577179 & -0.045108 \\ \mathrm{H}-1.636823 & -0.764760 & -0.978157 \\ \mathrm{H}-1.670373 & -1.003492 & 0.785251 \\ \mathrm{O} & 0.186482 & -1.212249 & -0.141137 \\ \mathrm{C} & 1.154047 & -0.214923 & 0.218910 \\ \mathrm{H} & 2.095099 & -0.458493 & -0.276943 \\ \mathrm{H} & 1.297278 & -0.203893 & 1.307830 \\ \mathrm{C} & -0.785404 & 0.921891 & 0.171010 \\ \mathrm{H} & -1.413818 & 1.590021 & -0.421565 \\ \mathrm{H} & -0.897522 & 1.192126 & 1.225056 \\ \mathrm{~N} & 0.629664 & 1.066110 & -0.195295 \\ \mathrm{H} & 0.689735 & 1.104975 & -1.214183\end{array}$

1,3-diphosphole-2-ylidene, singlet, trans

$\begin{array}{llcc}\mathrm{C} & -0.683384 & 1.318575 & -0.059436 \\ \mathrm{P} & -1.288554 & -0.346746 & 0.099323 \\ \mathrm{H} & -2.453930 & -0.595241 & -0.639949 \\ \mathrm{C} & -0.000151 & -1.441932 & -0.000510 \\ \mathrm{P} & 1.288537 & -0.346846 & -0.099041 \\ \mathrm{C} & 0.683569 & 1.318548 & 0.059198 \\ \mathrm{H} & -1.299409 & 2.211953 & -0.079244 \\ \mathrm{H} & 1.299692 & 2.211857 & 0.079011 \\ \mathrm{H} & 2.453693 & -0.595838 & 0.640447\end{array}$

1,3-diphosphole-2-ylidene, triplet, trans $\begin{array}{llll}C & -0.674133 & 1.243303 & -0.008597\end{array}$

$\begin{array}{llll}\mathrm{P}-1.557116 & -0.362206 & 0.108259\end{array}$

$\mathrm{H}-2.013790 \quad-0.475453 \quad-1.236499$

C $0.674138 \quad 1.243305 \quad 0.008589$

$\mathrm{H}-1.243524 \quad 2.170884 \quad-0.002870$

$\begin{array}{llll}\mathrm{H} & 1.243525 & 2.170888 & 0.002822\end{array}$

C $-0.000004 \quad-1.240713 \quad-0.000048$

$\begin{array}{llll}\mathrm{P} & 1.557124 & -0.362207 & -0.108237\end{array}$

$\mathrm{H} 2.013662-0.475489 \quad 1.236560$

1,3-diphosphole-2-CH+, trans

$\begin{array}{llcc}\text { H } & 1.289874 & 2.210957 & -0.083841 \\ \mathrm{C} & 0.683662 & 1.310601 & -0.061385 \\ \mathrm{C} & -0.683498 & 1.310632 & 0.061387 \\ \mathrm{H} & -1.289610 & 2.211058 & 0.083799 \\ \mathrm{C} & -0.000021 & -1.300595 & -0.000007 \\ \mathrm{H} & -0.000009 & -2.385922 & -0.000065 \\ \mathrm{P} & 1.380191 & -0.292028 & 0.102592 \\ \mathrm{H} & 2.478481 & -0.600214 & -0.711677 \\ \mathrm{P} & -1.380279 & -0.291951 & -0.102612 \\ \mathrm{H} & -2.478281 & -0.600020 & 0.712109\end{array}$

1,3-diphosphole-2-CH2, trans 


\begin{tabular}{|c|c|c|c|}
\hline $\mathrm{H}$ & 1.258905 & 2.233226 & 0.168859 \\
\hline $\mathrm{C}$ & 0.673954 & 1.317781 & 0.095978 \\
\hline $\mathrm{C}$ & -0.671483 & 1.329565 & 0.098195 \\
\hline $\mathrm{P}$ & 1.492876 & -0.276294 & -0.228245 \\
\hline $\mathrm{H}$ & -1.238736 & 2.251930 & 0.199912 \\
\hline $\mathrm{P}$ & -1.544318 & -0.275773 & -0.029184 \\
\hline $\mathrm{H}$ & 2.270153 & -0.363598 & 0.957108 \\
\hline $\mathrm{C}$ & 0.003238 & -1.269275 & 0.288404 \\
\hline $\mathrm{H}$ & 0.039186 & -1.508903 & 1.354134 \\
\hline $\mathrm{H}$ & -0.051077 & -2.209908 & -0.265264 \\
\hline $\mathrm{H}$ & -1.541067 & -0.390176 & -1.448771 \\
\hline & 5-dihydro- & , 3-diphosph & olane-2-ylidene, trans, singlet \\
\hline $\mathrm{H}$ & 0.678536 & 1.406083 & -1.377938 \\
\hline $\mathrm{C}$ & 0.710196 & 1.293683 & -0.288034 \\
\hline $\mathrm{P}$ & 1.340278 & -0.408444 & 0.180487 \\
\hline $\mathrm{C}$ & -0.710152 & 1.293688 & 0.288042 \\
\hline $\mathrm{H}$ & 1.326560 & 2.087590 & 0.138509 \\
\hline $\mathrm{P}$ & -1.340305 & -0.408419 & -0.180504 \\
\hline $\mathrm{H}$ & -1.326496 & 2.087618 & -0.138486 \\
\hline $\mathrm{H}$ & -0.678484 & 1.406070 & 1.377946 \\
\hline $\mathrm{C}$ & -0.000007 & -1.454352 & 0.000010 \\
\hline $\mathrm{H}$ & 2.388987 & -0.766298 & -0.690689 \\
\hline $\mathrm{H}$ & -2.388909 & -0.766231 & 0.690820 \\
\hline \multicolumn{4}{|c|}{ 4,5-dihydro-1,3-diphospholane, triplet, cis } \\
\hline $\mathrm{H}$ & 0.636207 & 1.282396 & -1.414834 \\
\hline $\mathrm{C}$ & 0.710842 & 1.187434 & -0.326115 \\
\hline $\mathrm{P}$ & 1.574151 & -0.438288 & 0.022874 \\
\hline $\mathrm{C}$ & -0.684289 & 1.213221 & 0.312936 \\
\hline $\mathrm{H}$ & 1.325844 & 2.020493 & 0.030434 \\
\hline $\mathrm{P}$ & -1.558718 & -0.385611 & -0.159602 \\
\hline $\mathrm{H}$ & -1.258859 & 2.075716 & -0.038702 \\
\hline $\mathrm{H}$ & -0.592236 & 1.284029 & 1.400587 \\
\hline $\mathrm{C}$ & -0.006424 & -1.284862 & -0.065214 \\
\hline $\mathrm{H}$ & -2.116834 & -0.724263 & 1.104492 \\
\hline $\mathrm{H}$ & 1.653612 & -0.274638 & 1.439307 \\
\hline \multicolumn{4}{|c|}{ 4,5-dihydro-1,3-diphospholane-2-C+, trans } \\
\hline $\mathrm{H}$ & 0.658078 & 1.359962 & -1.400984 \\
\hline $\mathrm{C}$ & 0.703664 & 1.284022 & -0.308269 \\
\hline $\mathrm{P}$ & 1.420606 & -0.350583 & 0.178866 \\
\hline $\mathrm{C}$ & -0.703539 & 1.283911 & 0.308217 \\
\hline $\mathrm{H}$ & 1.307513 & 2.103920 & 0.084051 \\
\hline$P$ & -1.420860 & -0.350583 & -0.178876 \\
\hline $\mathrm{H}$ & -1.307385 & 2.103882 & -0.083972 \\
\hline $\mathrm{H}$ & -0.657828 & 1.359824 & 1.400932 \\
\hline $\mathrm{H}$ & 2.420502 & -0.806994 & -0.701114 \\
\hline
\end{tabular}




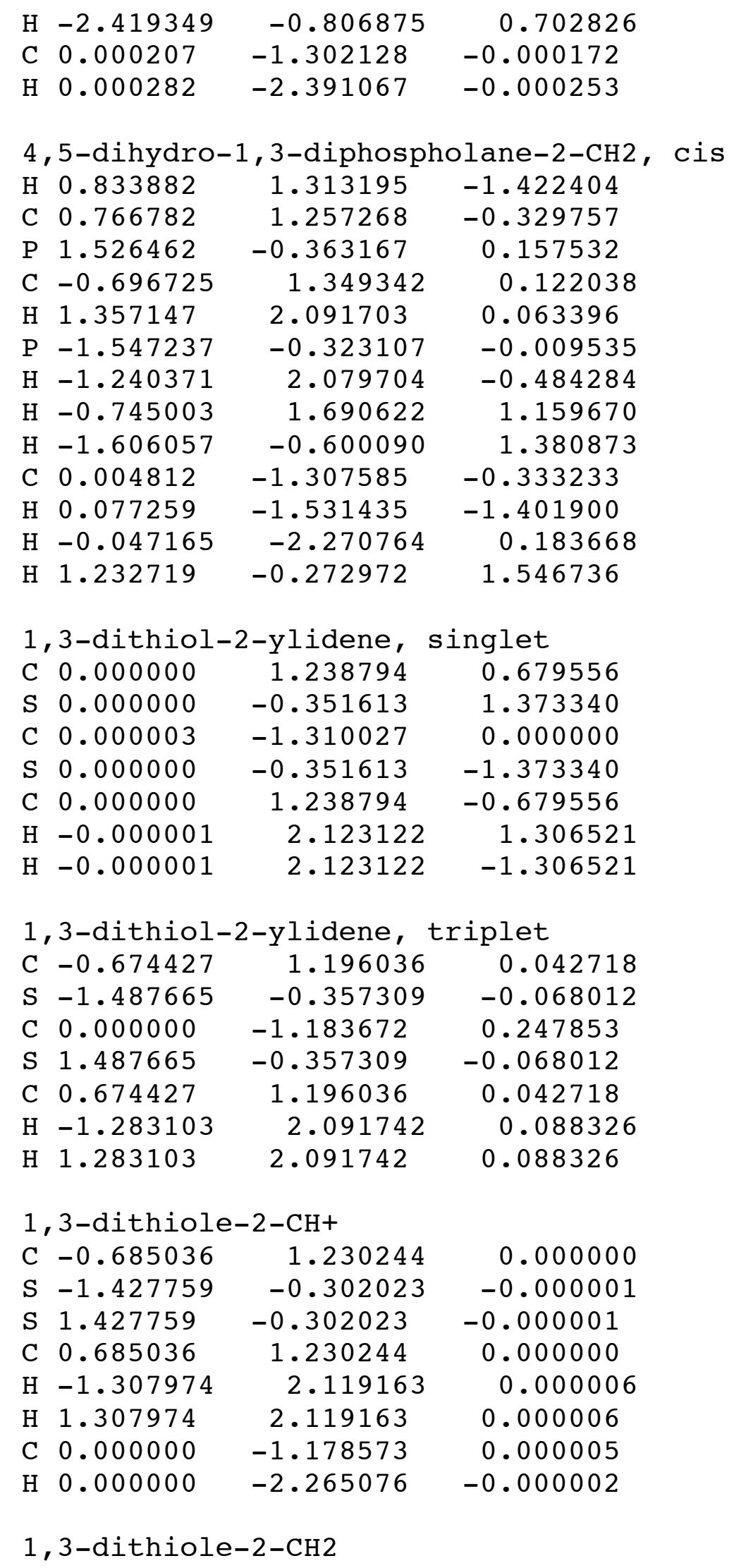




$\begin{array}{cccc}\mathrm{C} & 0.670913 & 1.270451 & 0.100786 \\ \mathrm{~S} & 1.484133 & -0.268918 & -0.140196 \\ \mathrm{~S}-1.484133 & -0.268919 & -0.140196 \\ \mathrm{C} & -0.670914 & 1.270450 & 0.100787 \\ \mathrm{H} & 1.279725 & 2.162275 & 0.195489 \\ \mathrm{H} & -1.279726 & 2.162275 & 0.195486 \\ \mathrm{C} & 0.000000 & -1.226760 & 0.297860 \\ \mathrm{H} & 0.000000 & -1.445410 & 1.368048 \\ \mathrm{H} & 0.000001 & -2.158584 & -0.269357\end{array}$

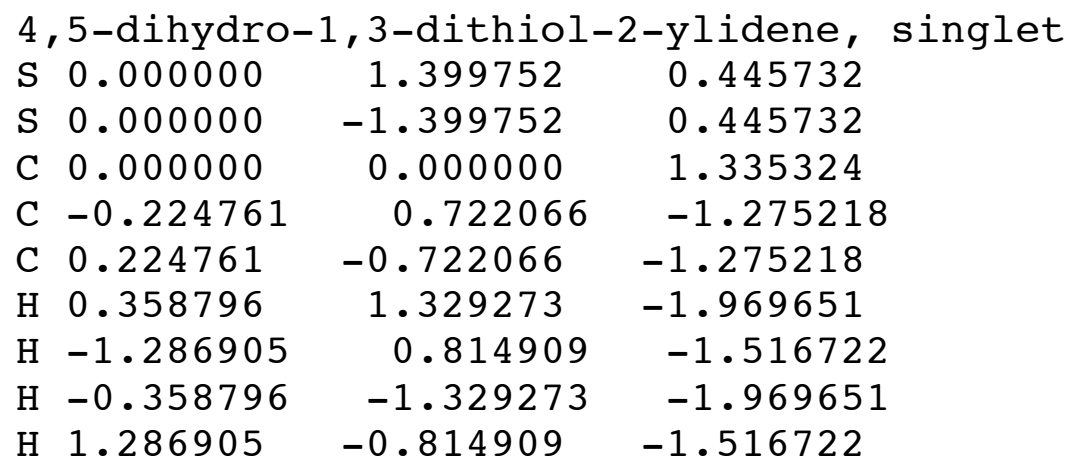

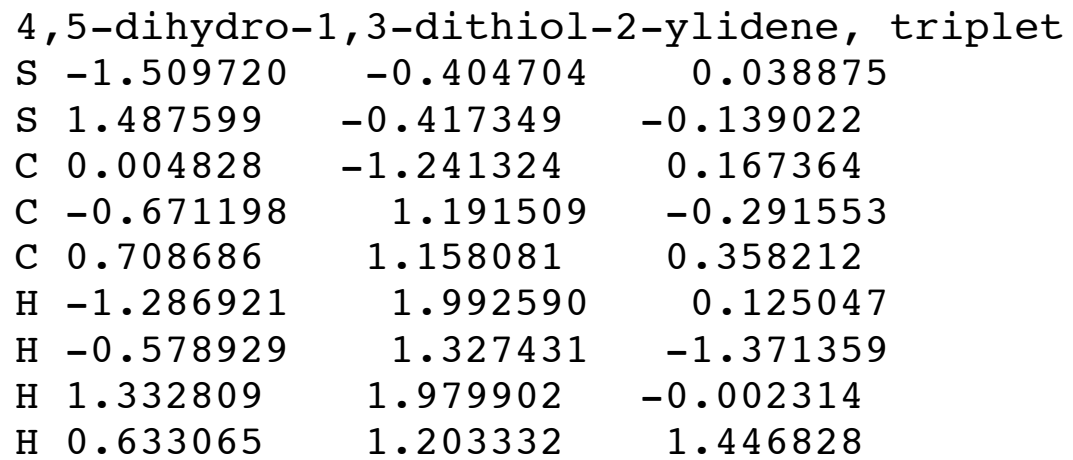

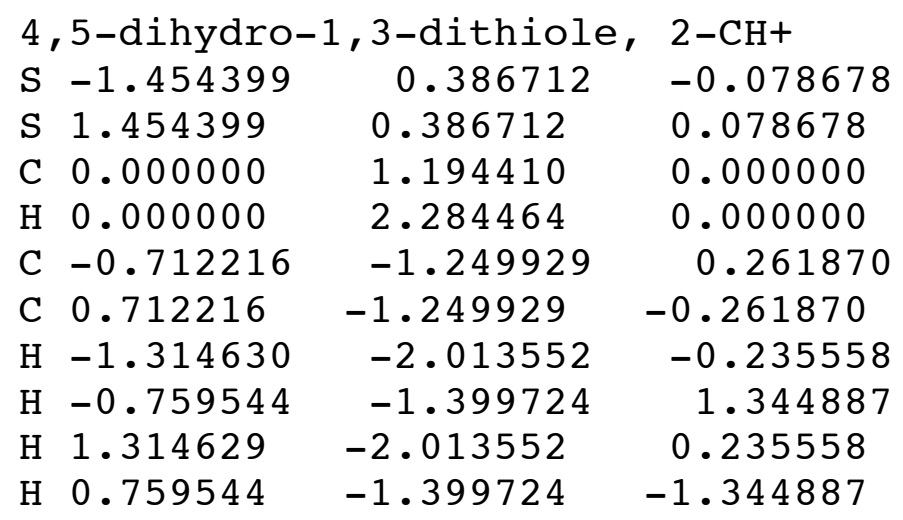

1,3-dithiolane, (4,5-dihydro-1.3-dithiole, 2-CH2)

$\begin{array}{llll}\mathrm{S} & 1.499419 & 0.305014 & 0.091478 \\ \mathrm{~S} & -1.499419 & 0.305014 & -0.091479 \\ \mathrm{C} & 0.000000 & 1.340778 & 0.000000\end{array}$ 


$\begin{array}{cccc}\mathrm{H} & 0.019258 & 1.973687 & -0.888495 \\ \mathrm{H} & -0.019258 & 1.973687 & 0.888496 \\ \mathrm{C} & 0.674824 & -1.246179 & -0.346406 \\ \mathrm{C} & -0.674824 & -1.246179 & 0.346406 \\ \mathrm{H} & 1.306661 & -2.064320 & 0.011929 \\ \mathrm{H} & 0.553408 & -1.334855 & -1.428484 \\ \mathrm{H} & -1.306661 & -2.064320 & -0.011929 \\ \mathrm{H} & -0.553407 & -1.334854 & 1.428484\end{array}$

$\mathrm{N}, \mathrm{N}^{\prime}$-dimethylimidazol-2-ylidene, singlet
$\mathrm{H}-1.389418$
2.030594
$-0.000003$
C -0.682355
1.213012
0.000007
C 0.682355
1.213012
0.000001
$\mathrm{N}-1.057934$
$-0.115809$
$-0.000002$
$\mathrm{H} 1.389418$
2.030594
$-0.000016$
N 1.057934
$-0.115808$
$-0.000015$
C -2.435371
$-0.571998$
0.000010
C 2.435371
$-0.571998$
0.000015
C 0.000000
$-0.990528$
$-0.000026$
$\mathrm{H}-2.406934$
$-1.660190$
$-0.000260$
H -2.959119
$-0.216894$
$-0.891189$
$\mathrm{H}-2.958956$
$-0.217351$
0.891487
H 2.958918
$-0.217439$
0.891549
H 2.959157
$-0.216805$
$-0.891127$
H 2.406934
$-1.660190$
$-0.000359$

\begin{tabular}{|c|c|c|c|}
\hline & $N^{\prime}-$ dimeth & midazol-2 & lidene, \\
\hline $\mathrm{H}$ & 1.355384 & 2.012971 & 0.313101 \\
\hline $\mathrm{C}$ & 0.675512 & 1.202140 & 0.082424 \\
\hline $\mathrm{C}$ & -0.675442 & 1.202142 & 0.082481 \\
\hline $\mathrm{H}$ & -1.355270 & 2.013015 & 0.313135 \\
\hline C & -0.000004 & -0.722402 & -0.751046 \\
\hline $\mathrm{N}$ & -1.177436 & -0.029230 & -0.400639 \\
\hline C & -2.194680 & -0.704991 & 0.407679 \\
\hline $\mathrm{H}$ & -2.543857 & -1.586817 & -0.131901 \\
\hline $\mathrm{H}$ & -3.039197 & -0.025883 & 0.545687 \\
\hline $\mathrm{H}$ & -1.809785 & -1.011112 & 1.389842 \\
\hline $\mathrm{N}$ & 1.177429 & -0.029257 & -0.400647 \\
\hline $\mathrm{C}$ & 2.194632 & -0.705028 & 0.407697 \\
\hline $\mathrm{H}$ & 1.809789 & -1.010840 & 1.389976 \\
\hline $\mathrm{H}$ & 3.039295 & -0.026046 & 0.545452 \\
\hline $\mathrm{H}$ & 2.543583 & -1.587050 & -0.131708 \\
\hline & $\mathrm{N}^{\prime}-$ dimeth & Limidazole- & $-\mathrm{CH}+$ \\
\hline $\mathrm{H}$ & 1.391350 & -2.049774 & 0.000009 \\
\hline $\mathrm{C}$ & 0.685079 & -1.232164 & 0.000016 \\
\hline $\mathrm{C}$ & -0.685079 & -1.232164 & -0.000015 \\
\hline $\mathrm{N}$ & 1.086971 & 0.081626 & 0.000017 \\
\hline
\end{tabular}




$\begin{array}{cccc}\mathrm{H}-1.391350 & -2.049774 & 0.000005 \\ \mathrm{~N}-1.086971 & 0.081626 & -0.000038 \\ \mathrm{C} & 2.478923 & 0.549246 & -0.000001 \\ \mathrm{C}-2.478923 & 0.549246 & 0.000022 \\ \mathrm{H} & 2.479756 & 1.638202 & 0.000344 \\ \mathrm{H} & 2.978641 & 0.180898 & -0.895564 \\ \mathrm{H} & 2.978833 & 0.180342 & 0.895224 \\ \mathrm{H} & -2.978621 & 0.180874 & 0.895586 \\ \mathrm{H}-2.978853 & 0.180365 & -0.895201 \\ \mathrm{H} & -2.479756 & 1.638202 & -0.000295 \\ \mathrm{C} & 0.000000 & 0.867462 & -0.000014 \\ \mathrm{H} & 0.000000 & 1.948149 & 0.000001 \\ & & & \\ \mathrm{~N}, \mathrm{~N}-\mathrm{dimethy} & \mathrm{M} \mathrm{i} \text { dazole-2 } & \mathrm{CH} 2 . \mathrm{Cis} \\ \mathrm{H}-1.353031 & 2.069093 & 0.155739 \\ \mathrm{C}-0.671849 & 1.235703 & 0.040505 \\ \mathrm{C} & 0.671848 & 1.235703 & 0.040504 \\ \mathrm{H} & 1.353032 & 2.069092 & 0.155742 \\ \mathrm{C} & -2.362050 & -0.513802 & 0.259719 \\ \mathrm{C} & 2.362050 & -0.513802 & 0.259719 \\ \mathrm{H} & -2.680773 & -1.441573 & -0.222927 \\ \mathrm{H} & -3.155618 & 0.225752 & 0.131907 \\ \mathrm{H} & -2.222663 & -0.706720 & 1.336511 \\ \mathrm{H} & 2.222664 & -0.706720 & 1.336511 \\ \mathrm{H} & 3.155618 & 0.225753 & 0.131906 \\ \mathrm{H} & 2.680773 & -1.441572 & -0.222928 \\ \mathrm{~N} & -1.151063 & -0.030785 & -0.380168 \\ \mathrm{~N} & 1.151063 & -0.030785 & -0.380167 \\ \mathrm{C} & 0.000000 & -0.910550 & -0.178539 \\ \mathrm{H} & 0.000000 & -1.309910 & 0.868008 \\ \mathrm{H} & 0.000000 & -1.751716 & -0.879579\end{array}$

\begin{tabular}{|c|c|c|c|}
\hline \multicolumn{4}{|c|}{ 4,5-dimethylimidazol-2-ylidene, singlet } \\
\hline $\mathrm{C}$ & 1.679858 & -1.371958 & -0.000049 \\
\hline $\mathrm{C}$ & 0.684422 & -0.264968 & 0.000055 \\
\hline $\mathrm{C}$ & -0.684421 & -0.264971 & -0.000123 \\
\hline $\mathrm{N}$ & 1.042775 & 1.076681 & 0.000055 \\
\hline $\mathrm{C}$ & -1.679852 & -1.371966 & 0.000076 \\
\hline $\mathrm{N}$ & -1.042780 & 1.076676 & -0.000056 \\
\hline $\mathrm{C}$ & -0.000004 & 1.965403 & 0.000021 \\
\hline $\mathrm{H}$ & 2.002912 & 1.396455 & 0.000158 \\
\hline $\mathrm{H}$ & -2.002918 & 1.396446 & -0.000138 \\
\hline $\mathrm{H}$ & 1.172329 & -2.339309 & -0.000140 \\
\hline $\mathrm{H}$ & 2.323749 & -1.334334 & 0.885072 \\
\hline $\mathrm{H}$ & 2.323783 & -1.334165 & -0.885136 \\
\hline $\mathrm{H}$ & -2.324125 & -1.334032 & -0.884753 \\
\hline $\mathrm{H}$ & -2.323396 & -1.334486 & 0.885455 \\
\hline $\mathrm{H}$ & -1.172318 & -2.339314 & -0.000395 \\
\hline
\end{tabular}




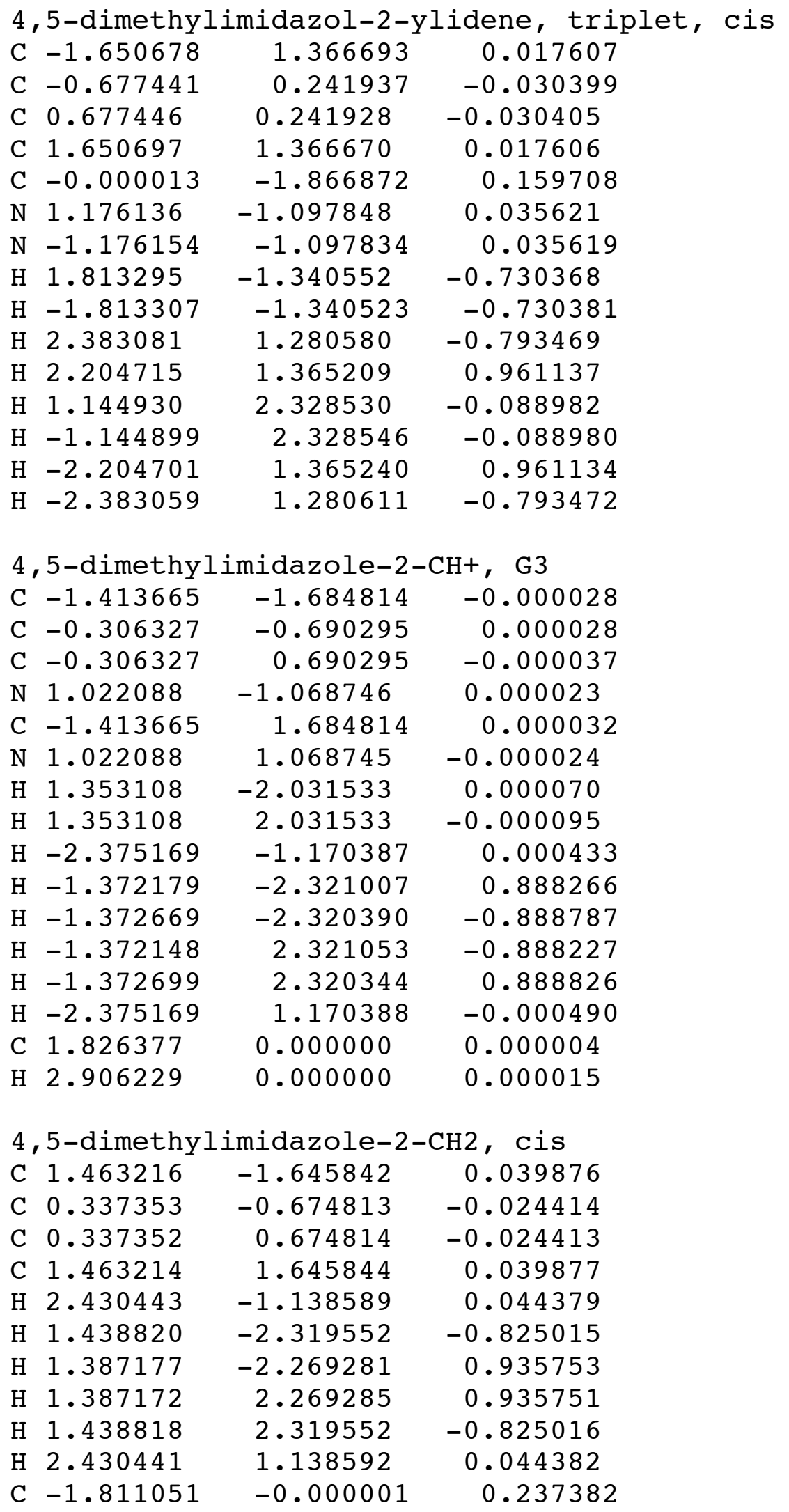




$\begin{array}{rrrr}\mathrm{H}-2.767203 & -0.000002 & -0.291694 \\ \mathrm{H}-1.996601 & -0.000001 & 1.316126 \\ \mathrm{~N}-1.005686 & 1.194263 & -0.067061 \\ \mathrm{~N}-1.005684 & -1.194265 & -0.067060 \\ \mathrm{H}-1.204989 & 1.562296 & -1.002837 \\ \mathrm{H}-1.204987 & -1.562298 & -1.002835\end{array}$

4,5-difluoroimidazol-2-ylidene, singlet
F 0.000000
1.556111
$-1.331894$
C 0.000000
0.678485
$-0.324793$
C 0.000000
$-0.678485$
$-0.324793$
F 0.000000
$-1.556111$
$-1.331894$
$\mathrm{N} 0.000000$
1.053010
0.997627
$\mathrm{N} 0.000000$
$-1.053010$
0.997627
C 0.000000
0.000000
1.882765
$\mathrm{H} 0.000000$
2.017662
1. 304117
$\mathrm{H} 0.000000$
$-2.017662$
1. 304117

\begin{tabular}{|c|c|c|c|}
\hline \multicolumn{4}{|c|}{ 4,5-difluoroimidazol-2-ylidene, triplet } \\
\hline $\mathrm{C}$ & 0.000002 & 1.774357 & 0.000000 \\
\hline $\mathrm{N}$ & -0.963208 & 0.915908 & 0.467418 \\
\hline $\mathrm{H}$ & -1.821275 & 1.261547 & 0.875188 \\
\hline $\mathrm{N}$ & 0.963209 & 0.915907 & -0.467418 \\
\hline $\mathrm{H}$ & 1.821279 & 1.261544 & -0.875184 \\
\hline $\mathrm{C}$ & 0.691286 & -0.442238 & -0.279537 \\
\hline $\mathrm{C}$ & -0.691287 & -0.442237 & 0.279537 \\
\hline $\mathrm{F}$ & 1.628202 & -1.149173 & 0.402963 \\
\hline $\mathrm{F}$ & -1.628204 & -1.149170 & -0.402962 \\
\hline
\end{tabular}

\begin{tabular}{|c|c|c|}
\hline \multicolumn{3}{|c|}{4,5 -difluoroimidazol-2-CH+ } \\
\hline $\mathrm{F} 0.000000$ & 1.550875 & -1.357285 \\
\hline 0.000000 & 0.685114 & -0.374505 \\
\hline 0.000000 & -0.685114 & -0.374505 \\
\hline 0.000000 & -1.550875 & -1.357285 \\
\hline 0.000000 & 1.075778 & 0.939572 \\
\hline 0.000000 & -1.075778 & 0.939572 \\
\hline 0.000000 & 2.047644 & 1.252244 \\
\hline 0.000000 & -2.047644 & 1.252244 \\
\hline 0.000000 & 0.000000 & 1.740978 \\
\hline 0.000000 & 0.000000 & 2.820832 \\
\hline
\end{tabular}
4,5-difluoroimidazole-2-CH2, cis
$\begin{array}{lll}\mathrm{F}-1.426490 & -1.497122 & 0.050627\end{array}$
$\begin{array}{lll}\text { C }-0.377677 & -0.669933 & -0.032878\end{array}$
C $-0.377673 \quad 0.669935 \quad-0.032873$
$\begin{array}{lll}\mathrm{F}-1.426481 & 1.497129 & 0.050627\end{array}$
C $1.718950 \quad-0.000004 \quad 0.263459$
$\mathrm{H} 2.692559-0.000008-0.227856$ 


$\begin{array}{rrrr}\mathrm{H} & 1.846718 & -0.000004 & 1.348229 \\ \mathrm{~N} & 0.932800 & 1.205099 & -0.083747 \\ \mathrm{~N} & 0.932793 & -1.205105 & -0.083745 \\ \mathrm{H} & 1.148352 & -1.554708 & -1.022737 \\ \mathrm{H} & 1.148361 & 1.554714 & -1.022733\end{array}$

$1,3,4,5$-tetramethylimidazol-2-ylidene, singlet

$\begin{array}{lll}\text { C }-1.668857 & 1.754568 & -0.000084\end{array}$

C $-0.685941 \quad 0.636139 \quad-0.000054$

$\begin{array}{lll}\text { C } 0.685941 & 0.636140 & 0.000020\end{array}$

$\begin{array}{lll}\mathrm{N}-1.055487 & -0.701370 & -0.000107\end{array}$

C $1.668856 \quad 1.754569 \quad 0.000100$

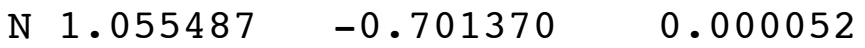

$\begin{array}{lll}\text { C } 0.000000 & -1.577088 & -0.000015\end{array}$

$\begin{array}{lll}\text { C }-2.429057 & -1.164788 & 0.000119\end{array}$

$\begin{array}{lll}\text { C } 2.429058 & -1.164788 & -0.000067\end{array}$

$\mathrm{H}-2.960670 \quad-0.815786 \quad-0.889749$

$\mathrm{H}-2.959838 \quad-0.817543 \quad 0.891185$

$\mathrm{H}-2.389470 \quad-2.252912 \quad-0.000994$

H $2.960488 \quad-0.816154 \quad 0.890057$

H $2.960020 \quad-0.817174 \quad-0.890878$

H $2.389470 \quad-2.252913 \quad 0.000579$

$\begin{array}{llll}\mathrm{H} & 2.315520 & 1.728725 & -0.883745\end{array}$

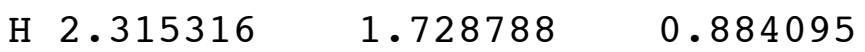

$\mathrm{H} 1.146132 \quad 2.714060 \quad 0.000007$

$\mathrm{H}-1.146133 \quad 2.714059 \quad 0.000055$

$\mathrm{H}-2.315529 \quad 1.728686 \quad 0.883754$

$\mathrm{H}-2.315309 \quad 1.728824 \quad-0.884086$

\begin{tabular}{|c|c|c|}
\hline C 1.638629 & 1.760922 & 0.083189 \\
\hline C 0.679232 & 0.635542 & -0.100047 \\
\hline$C-0.679252$ & 0.635532 & -0.100071 \\
\hline 1.176849 & -0.657536 & -0.436831 \\
\hline-1.638674 & 1.760891 & 0.083150 \\
\hline-1.176852 & -0.657568 & -0.436846 \\
\hline 0.000013 & -1.377175 & -0.715974 \\
\hline 2.155962 & -1.259812 & 0.473570 \\
\hline-2.155912 & -1.259851 & 0.473617 \\
\hline 1.760720 & -1.377906 & 1.492732 \\
\hline 3.053821 & -0.639213 & 0.501412 \\
\hline H 2.431116 & -2.240631 & 0.082402 \\
\hline-2.431050 & -2.240690 & 0.082489 \\
\hline-3.053789 & -0.639278 & 0.501475 \\
\hline $\mathrm{H}-1.760624$ & -1.377900 & 1.492766 \\
\hline-2.254254 & 1.630010 & 0.979593 \\
\hline-2.312815 & 1.833541 & -0.776158 \\
\hline
\end{tabular}




$\begin{array}{cccc}\mathrm{H} & -1.106570 & 2.708921 & 0.185429 \\ \mathrm{H} & 1.106500 & 2.708934 & 0.185503 \\ \mathrm{H} & 2.312756 & 1.833614 & -0.776127 \\ \mathrm{H} & 2.254224 & 1.630031 & 0.979621\end{array}$

$1,3,4,5$-tetramethylimidazole-2-CH+

C $1.673154 \quad-1.779256 \quad-0.000011$

$\begin{array}{llll}\text { C } 0.690756 & -0.661678 & -0.000008\end{array}$

$\begin{array}{lll}\mathrm{C}-0.690756 & -0.661678 & 0.000002\end{array}$

$\begin{array}{llll}\mathrm{N} & 1.083671 & 0.663031 & -0.000006\end{array}$

C $-1.673154 \quad-1.779256 \quad 0.000014$

$\begin{array}{lll}\mathrm{N}-1.083671 & 0.663031 & 0.000004\end{array}$

C $2.468523 \quad 1.139157 \quad 0.000012$

$\begin{array}{lll}\text { C }-2.468524 & 1.139157 & -0.000010\end{array}$

$\mathrm{H} \quad 2.977100 \quad 0.777345 \quad-0.893791$

H $2.977032 \quad 0.777473 \quad 0.893907$

$\begin{array}{llll}\mathrm{H} & 2.460257 & 2.228341 & -0.000072\end{array}$

$\mathrm{H}-2.977087 \quad 0.777376 \quad 0.893814$

$\mathrm{H}-2.977046 \quad 0.777442 \quad-0.893885$

$\mathrm{H}-2.460257 \quad 2.228341 \quad 0.000034$

$\mathrm{H}-2.312384 \quad-1.749868 \quad-0.887127$

$\mathrm{H}-2.312379 \quad-1.749852 \quad 0.887157$

$\mathrm{H}-1.146962 \quad-2.734534 \quad 0.000021$

$\mathrm{H} 1.146962-2.734533 \quad-0.000052$

H $2.312359-1.749884 \quad 0.887149$

H $2.312405 \quad-1.749835 \quad-0.887135$

C $0.000000 \quad 1.451499 \quad 0.000000$

$\mathrm{H} 0.000000 \quad 2.532081 \quad 0.000005$

$1,3,4,5$-tetramethylimidazol-2-CH2, cis

$\begin{array}{lll}\text { C } 1.879697 & 1.580861 & -0.435028\end{array}$

$\begin{array}{lll}\text { C } 0.806404 & 0.565846 & -0.256566\end{array}$

$\begin{array}{lll}C-0.486702 & 0.706740 & 0.111339\end{array}$

$\mathrm{N} 1.132168-0.836760 \quad-0.396306$

$\begin{array}{lll}\mathrm{C}-1.256984 & 1.939672 & 0.432987\end{array}$

$\mathrm{N}-1.092275 \quad-0.574120 \quad 0.266870$

C $1.846578 \quad-1.316994 \quad 0.797106$

$\begin{array}{llll}\text { C }-2.479709 & -0.704634 & -0.141555\end{array}$

$\mathrm{H} 1.290387 \quad-1.135438 \quad 1.728242$

H $2.813201 \quad-0.812486 \quad 0.858777$

H $2.035268 \quad-2.388487 \quad 0.691196$

$\mathrm{H}-2.629056-0.492534 \quad-1.214589$

$\mathrm{H}-3.111415 \quad-0.033637 \quad 0.443417$

$\mathrm{H}-2.807605 \quad-1.729014 \quad 0.054856$

$\mathrm{H}-1.674012 \quad 1.869976 \quad 1.443481$

$\mathrm{H}-2.091634 \quad 2.099276 \quad-0.258157$

$\mathrm{H}-0.612287 \quad 2.819492 \quad 0.391634$

$\begin{array}{llll}\mathrm{H} & 1.474213 & 2.594830 & -0.401318\end{array}$ 


$\begin{array}{llll}\mathrm{H} & 2.374300 & 1.437840 & -1.400722 \\ \mathrm{H} & 2.651301 & 1.505869 & 0.340444 \\ \mathrm{C}-0.192287 & -1.434655 & -0.514343 \\ \mathrm{H}-0.202785 & -2.468389 & -0.155208 \\ \mathrm{H}-0.491118 & -1.412163 & -1.579640\end{array}$

$1,3,4,5$-tetrasilylimidazol-2-ylidene singlet

Si $1.927033 \quad 1.983223 \quad 0.000081$

$\begin{array}{llrr}\text { C } & 0.692367 & 0.576637 & -0.000018 \\ \text { C }-0.692366 & 0.576637 & 0.000000\end{array}$

$\mathrm{N} 1.071641 \quad-0.778322 \quad-0.000054$

$\mathrm{Si}-1.927036 \quad 1.983220 \quad-0.000072$

$\mathrm{N}-1.071639 \quad-0.778324 \quad-0.000018$

C $0.000002 \quad-1.632465 \quad-0.000047$

Si $2.651469 \quad-1.610424 \quad-0.000034$

Si $-2.651468 \quad-1.610425 \quad 0.000072$

$\mathrm{H} 2.782279 \quad-2.433457 \quad 1.219227$

$\mathrm{H} 2.781791-2.434313-1.218759$

$\mathrm{H} 3.684488 \quad-0.547028-0.000600$

$\mathrm{H} 2.790470 \quad 1.905864 \quad-1.205324$

$\mathrm{H} 1.198245 \quad 3.270390 \quad 0.000567$

$\mathrm{H} 2.790899 \quad 1.905230 \quad 1.205138$

$\mathrm{H}-1.198253 \quad 3.270390 \quad-0.000464$

$\mathrm{H}-2.790859 \quad 1.905285-1.205164$

$\mathrm{H}-2.790515 \quad 1.905796 \quad 1.205299$

$\mathrm{H}-2.782215 \quad-2.433692 \quad-1.219034$

$\mathrm{H}-2.781850 \quad-2.434082 \quad 1.218951$

$\mathrm{H}-3.684487 \quad-0.547029 \quad 0.000397$

$1,3,4,5$-tetrasilylimidazol-2-ylidene, triplet, trans

$\begin{array}{lcc}\mathrm{Si}-1.948650 & 1.781125 & -0.888533 \\ \mathrm{C}-0.770194 & 0.601543 & -0.075814 \\ \mathrm{C} 0.738543 & 0.667477 & -0.084685 \\ \mathrm{~N}-1.019226 & -0.755611 & 0.252220 \\ \mathrm{Si} 1.741062 & 1.864957 & 0.936532 \\ \mathrm{~N} 1.103300 & -0.695600 & -0.303029 \\ \mathrm{C} 0.078888 & -1.559789 & 0.017863 \\ \mathrm{Si}-2.548447 & -1.592919 & 0.609360 \\ \mathrm{Si} 2.710208 & -1.402659 & -0.596510 \\ \mathrm{H}-2.311135 & -2.601285 & 1.660583 \\ \mathrm{H}-3.482384 & -0.547061 & 1.089814 \\ \mathrm{H}-3.100904 & -2.231958 & -0.607494 \\ \mathrm{H}-3.123442 & 1.031186 & -1.403558 \\ \mathrm{H}-2.438247 & 2.821616 & 0.054725 \\ \mathrm{H}-1.229995 & 2.465279 & -1.995136 \\ \mathrm{H} 1.496898 & 3.243805 & 0.446063 \\ \mathrm{H} 1.357036 & 1.811631 & 2.376408 \\ \mathrm{H} 3.179302 & 1.529307 & 0.792981\end{array}$




\begin{tabular}{|c|c|c|}
\hline 2.530481 & -2.666312 & -1.334849 \\
\hline $\mathrm{H} 3.468913$ & -0.417439 & -1.401539 \\
\hline & -1.642735 & \\
\hline \multicolumn{3}{|c|}{$1,3,4,5$-tetrasilylimidazole-2-CH+ } \\
\hline $\mathrm{Si} 1.874100$ & 1.998527 & 0.006952 \\
\hline 0.694458 & 0.511114 & 0.000979 \\
\hline-0.694460 & 0.511113 & -0.000980 \\
\hline 1.100064 & -0.825543 & 0.003260 \\
\hline$i-1.874100$ & 1.998525 & -0.006951 \\
\hline-1.100063 & -0.825545 & -0.003263 \\
\hline Si 2.809563 & -1.511209 & -0.005087 \\
\hline $\mathrm{Si}-2.809563$ & -1.511208 & 0.005089 \\
\hline Н 2.568617 & -2.947742 & 0.213589 \\
\hline $\mathrm{H} \quad 3.387456$ & -1.208058 & -1.323884 \\
\hline H 3.507168 & -0.849346 & 1.108641 \\
\hline H 3.127827 & 1.542515 & -0.630449 \\
\hline H 1.220587 & 3.059555 & -0.784088 \\
\hline Н 2.113374 & 2.422115 & 1.403124 \\
\hline $\mathrm{H}-1.220533$ & 3.059588 & 0.783998 \\
\hline-2.113456 & 2.422048 & -1.403130 \\
\hline $\mathrm{H}-3.127791$ & 1.542550 & 0.630545 \\
\hline-3.507174 & -0.849329 & -1.108627 \\
\hline-2.568624 & -2.947739 & -0.213605 \\
\hline-3.387447 & -1.208068 & 1.323892 \\
\hline 0.000000 & -1.596631 & -0.000001 \\
\hline $\mathrm{H} \quad 0.000001$ & -2.678947 & -0.000002 \\
\hline
\end{tabular}

$1,3,4,5$-tetrasilylimidazol-2-CH2, cis

$\begin{array}{lll}\text { Si }-2.147461 & 1.800921 & 0.087514\end{array}$

$\begin{array}{lll}\text { C }-0.798889 & 0.513269 & 0.206740\end{array}$

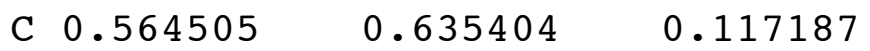

Si $1.622864 \quad 2.136599 \quad-0.236433$

Si $2.660074-1.280048 \quad-0.234224$

$\mathrm{H}-3.033998 \quad 1.700500 \quad 1.274267$

$\mathrm{H}-1.543862 \quad 3.152019 \quad 0.030047$

$\mathrm{H}-2.978090 \quad 1.594589 \quad-1.127747$

$\mathrm{H} \quad 0.807168 \quad 3.220040 \quad-0.827184$

H $2.296962 \quad 2.627326 \quad 0.993772$

$\mathrm{H} 2.674022 \quad 1.731987 \quad-1.205142$

H $3.838150 \quad-0.524797 \quad 0.261312$

H $2.704241 \quad-2.670707 \quad 0.278509$

$\mathrm{H} 2.726734-1.293812 \quad-1.720638$

C $0.115277 \quad-1.395540 \quad 1.064033$

$\mathrm{H} 0.148807 \quad-1.190034 \quad 2.147299$

$\mathrm{H} 0.215397 \quad-2.470769 \quad 0.895906$

$\mathrm{N} 1.168328 \quad-0.619760 \quad 0.395457$

$\mathrm{N}-1.129706 \quad-0.851501 \quad 0.524242$ 


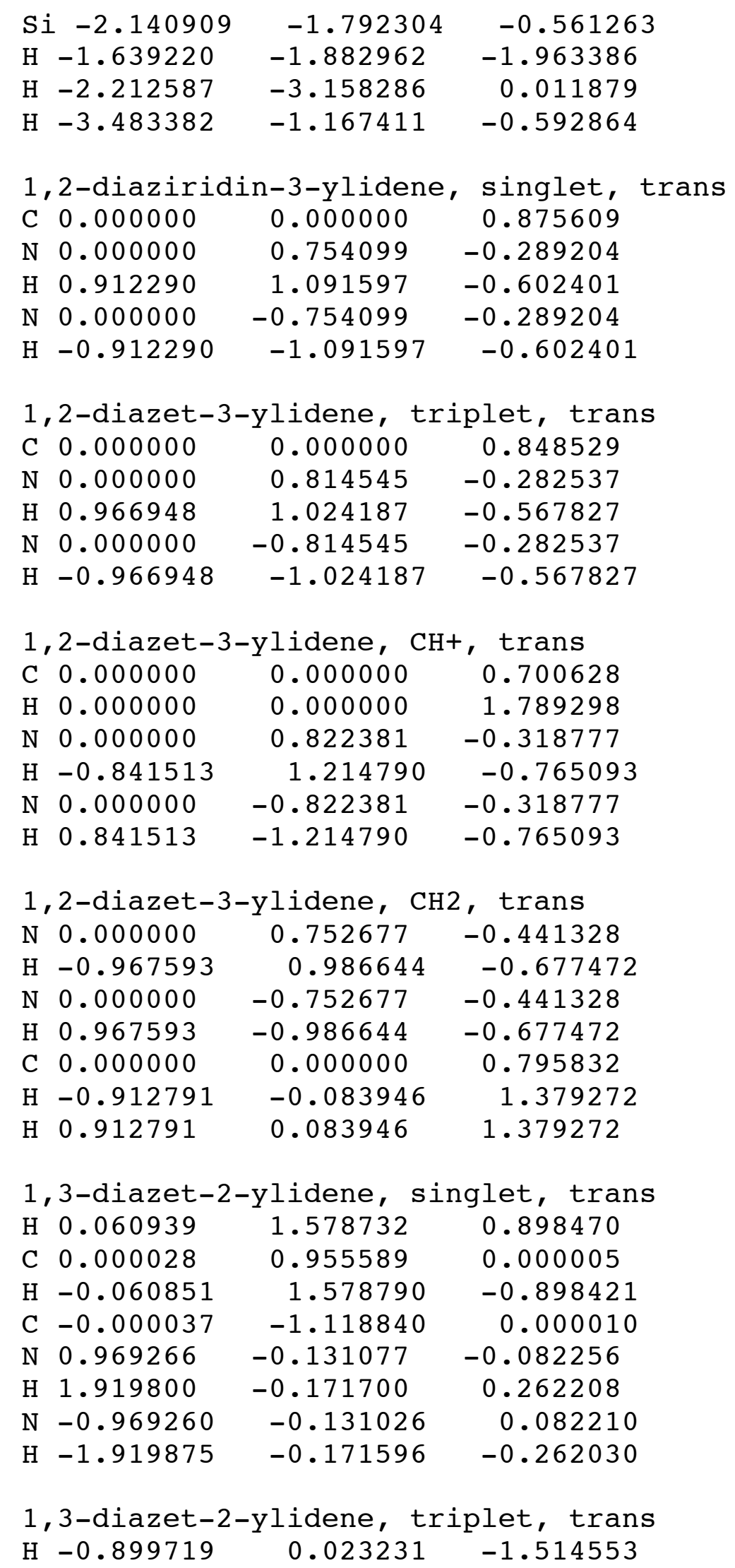




\begin{tabular}{|c|c|c|c|}
\hline C & 0.000000 & 0.000000 & -0.893989 \\
\hline $\mathrm{H}$ & 0.899719 & -0.023231 & -1.514553 \\
\hline $\mathrm{N}$ & 0.000000 & -1.087934 & 0.127885 \\
\hline $\mathrm{N}$ & 0.000000 & 1.087934 & 0.127885 \\
\hline $\mathrm{C}$ & 0.000000 & 0.000000 & 1.057982 \\
\hline $\mathrm{H}$ & 0.865981 & 1.630652 & 0.127383 \\
\hline $\mathrm{H}$ & -0.865981 & -1.630652 & 0.127383 \\
\hline \multicolumn{4}{|c|}{ 1,3-diazet-2-ylidene-2-CH+, trans } \\
\hline $\mathrm{H}$ & -0.042402 & 1.607280 & -0.912536 \\
\hline $\mathrm{C}$ & -0.000011 & 1.011860 & 0.000000 \\
\hline $\mathrm{H}$ & 0.042368 & 1.607278 & 0.912540 \\
\hline $\mathrm{C}$ & 0.000011 & -0.965884 & 0.000001 \\
\hline $\mathrm{H}$ & 0.000023 & -2.050840 & 0.000001 \\
\hline $\mathrm{N}$ & -0.991529 & -0.082715 & 0.052256 \\
\hline $\mathrm{H}$ & -1.982742 & -0.140880 & -0.163424 \\
\hline $\mathrm{N}$ & 0.991530 & -0.082693 & -0.052255 \\
\hline $\mathrm{H}$ & 1.982747 & -0.140839 & 0.163409 \\
\hline \multicolumn{3}{|c|}{1,3 -diazet-2-ylidene-CH2 } & cis-diequatorial \\
\hline $\mathrm{H}$ & 1.076326 & -0.285092 & 1.388763 \\
\hline $\mathrm{C}$ & 0.070814 & -0.018757 & 1.017336 \\
\hline $\mathrm{N}$ & 0.070814 & 1.052866 & 0.000000 \\
\hline $\mathrm{H}$ & -0.593137 & 0.157107 & 1.869805 \\
\hline $\mathrm{N}$ & -0.459663 & -0.949871 & 0.000000 \\
\hline $\mathrm{H}$ & -0.008893 & -1.863408 & 0.000000 \\
\hline $\mathrm{H}$ & 0.914690 & 1.623495 & 0.000000 \\
\hline $\mathrm{C}$ & 0.070814 & -0.018757 & -1.017336 \\
\hline $\mathrm{H}$ & 1.076326 & -0.285092 & -1.388763 \\
\hline $\mathrm{H}$ & -0.593137 & 0.157107 & -1.869805 \\
\hline \multicolumn{4}{|c|}{ azet-2-ylidene, singlet } \\
\hline $\mathrm{H}$ & 0.139954 & 1.615457 & 0.754038 \\
\hline $\mathrm{C}$ & 0.016277 & 0.811901 & 0.022354 \\
\hline $\mathrm{C}$ & -1.088577 & -0.027400 & -0.168332 \\
\hline $\mathrm{H}$ & -2.158506 & 0.059578 & -0.086090 \\
\hline $\mathrm{C}$ & -0.110262 & -0.918604 & 0.277861 \\
\hline $\mathrm{N}$ & 1.039998 & -0.113869 & -0.263964 \\
\hline $\mathrm{H}$ & 1.833936 & -0.073339 & 0.388501 \\
\hline \multicolumn{4}{|c|}{ azet-2-ylidene, triplet } \\
\hline $\mathrm{H}$ & 0.487873 & 1.969812 & 0.000335 \\
\hline $\mathrm{C}$ & 0.164899 & 0.936145 & -0.000058 \\
\hline $\mathrm{C}$ & -1.010071 & 0.130259 & 0.000030 \\
\hline $\mathrm{H}$ & -2.068561 & 0.358516 & -0.000097 \\
\hline $\mathrm{C}$ & -0.254491 & -1.137823 & 0.000052 \\
\hline $\mathrm{N}$ & 0.896889 & -0.215932 & -0.000098 \\
\hline $\mathrm{H}$ & 1.900446 & -0.388286 & 0.000302 \\
\hline
\end{tabular}




\begin{tabular}{|c|c|c|c|}
\hline \multicolumn{4}{|c|}{ azet-2-ylidene-2-CH+ } \\
\hline $\mathrm{H}$ & $1.3243<0$ & $-1.5 / 8279$ & 0.000002 \\
\hline & 0.612354 & -0.756075 & 0.000000 \\
\hline & -0.898828 & -0.577315 & 0.000000 \\
\hline 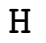 & -1.747960 & -1.244493 & 0.000000 \\
\hline $\mathrm{N}$ & 0.844157 & 0.509571 & -0.000002 \\
\hline $\mathrm{H}$ & 1.701696 & 1.065836 & 0.000005 \\
\hline 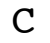 & -0.710138 & 0.747002 & 0.000001 \\
\hline & -1.207481 & 1.708264 & -0.000001 \\
\hline \multicolumn{4}{|c|}{ azet-2-ylidene-2-CH2 } \\
\hline $\mathrm{H}$ & 1.768147 & -1.099497 & 0.002840 \\
\hline & 0.886182 & -0.465444 & 0.012008 \\
\hline . & 0.621071 & 0.856629 & 0.039066 \\
\hline $\mathrm{H}$ & 1.203129 & 1.764038 & -0.046895 \\
\hline & -0.464380 & -0.910134 & -0.140574 \\
\hline & -0.812047 & -1.516080 & 0.606246 \\
\hline & -0.853697 & 0.538921 & 0.006291 \\
\hline & -1.404077 & 0.741338 & 0.930693 \\
\hline & -1.425824 & 0.900505 & -0.853055 \\
\hline
\end{tabular}

\begin{tabular}{|c|c|c|}
\hline \multicolumn{3}{|c|}{ azetidine-2-ylidene, singlet } \\
\hline H 0.023397 & 1.622731 & 0.894740 \\
\hline 0.040999 & 0.992312 & -0.000005 \\
\hline-0.960118 & -0.070598 & 0.000007 \\
\hline 1.035221 & -0.195795 & 0.000004 \\
\hline 0.023391 & 1.622705 & -0.894769 \\
\hline 1.665443 & -0.280562 & 0.889312 \\
\hline 1.665496 & -0.280552 & -0.889268 \\
\hline-0.189313 & -1.158112 & -0.000012 \\
\hline-1.978345 & -0.020568 & 0.000010 \\
\hline
\end{tabular}

\begin{tabular}{lcc}
\multicolumn{3}{l}{ azetidine-2-ylidene, triplet } \\
H -0.132611 & 1.449722 & 0.971071 \\
C -0.056220 & 0.920289 & 0.017372 \\
N -1.083925 & -0.156732 & -0.146713 \\
C 1.108005 & -0.107908 & -0.000290 \\
H -0.071710 & 1.630584 & -0.812451 \\
H 1.775258 & -0.072364 & 0.865769 \\
H 1.707587 & -0.110289 & -0.916009 \\
C -0.027889 & -1.086783 & 0.045154 \\
H -1.834424 & -0.154122 & 0.545195
\end{tabular}

$\begin{array}{lcc}\text { azetidine-2-ylidene-2-CH+ } \\ \text { H } 0.616504 & 1.534135 & 0.902418 \\ \mathrm{C} 0.432320 & 0.950697 & -0.000013 \\ \mathrm{~N}-0.928267 & 0.338869 & 0.000012\end{array}$




$\begin{array}{clcc}\mathrm{C} & 0.946111 & -0.518988 & 0.000010 \\ \mathrm{H} & 0.616468 & 1.534078 & -0.902489 \\ \mathrm{H} & 1.493622 & -0.831477 & 0.893048 \\ \mathrm{H} & 1.493724 & -0.831510 & -0.892952 \\ \mathrm{H} & -1.878115 & 0.723483 & 0.000040 \\ \mathrm{C} & -0.502663 & -0.883088 & -0.000015 \\ \mathrm{H} & -1.098945 & -1.792517 & -0.000044\end{array}$

\begin{tabular}{|c|c|c|}
\hline \multicolumn{3}{|c|}{ azetidine-2-ylidene-2-CH2 } \\
\hline H 1.932507 & -0.049373 & -0.522144 \\
\hline 1.042258 & -0.038288 & 0.114178 \\
\hline 0.000000 & -1.022700 & -0.261761 \\
\hline 0.000000 & 1.072675 & -0.097669 \\
\hline 1.354305 & -0.095081 & 1.167625 \\
\hline 0.000000 & 1.436478 & -1.126523 \\
\hline 0.000000 & 1.916237 & 0.594738 \\
\hline 0.000000 & -1.881497 & 0.289031 \\
\hline-1.042258 & -0.038288 & 0.114178 \\
\hline-1.932507 & -0.049373 & -0.522144 \\
\hline-1.354305 & -0.095081 & 1.167625 \\
\hline
\end{tabular}

\section{$\underline{\text { miscellaneous carbenes }}$}

diethynylcarbene, singlet
$\mathrm{H} 0.000000$
3.305911
$-0.818063$
C 0.000000
2.357864
$-0.327570$
C 0.000000
1.195025
0.082412
C 0.000000
0.000000
0.763005
C 0.000000
$-1.195025$
0.082412
C 0.000000
$-2.357864$
$-0.327570$
$\mathrm{H} 0.000000$
$-3.305911$
$-0.818063$

diethynylcarbene, triplet
$\mathrm{H} 0.000000$
3.598364
$-0.000593$
C 0.000000
2. 532986
$-0.000235$
C 0.000000
1. 314889
$-0.000031$
C 0.000000
0.000000
0.000731
C 0.000000
$-1.314889$
$-0.000031$
C 0.000000
$-2.532986$
$-0.000235$
$\mathrm{H} 0.000000$
$-3.598364$
$-0.000593$

diethynylmethyl+
$\mathrm{H} 0.000000$
3.267429
$-0.950295$
C 0.000000
2. 311702
$-0.457416$
C 0.000000
1.210040
0.087295
C 0.000000
0.000000
0.749594
C 0.000000
$-1.210040$
0.087295
$\mathrm{H} 0.000000$
0.000000
1.844477 


\begin{tabular}{|c|c|c|c|}
\hline C & 0.000000 & -2.311702 & -0.457416 \\
\hline $\mathrm{H}$ & 0.000000 & -3.267429 & -0.950295 \\
\hline & Lethynylmet & nane & \\
\hline $\mathrm{H}$ & 0.000000 & 3.148171 & -1.159204 \\
\hline C & 0.000000 & 2.261239 & -0.567661 \\
\hline $\mathrm{C}$ & 0.000000 & 1.220376 & 0.065414 \\
\hline $\mathrm{C}$ & 0.000000 & -1.220376 & 0.065414 \\
\hline C & 0.000000 & -2.261239 & -0.567661 \\
\hline $\mathrm{H}$ & 0.000000 & -3.148171 & -1.159204 \\
\hline C & 0.000000 & 0.000000 & 0.878667 \\
\hline $\mathrm{H}$ & 0.877665 & 0.000000 & 1.536681 \\
\hline $\mathrm{H}$ & -0.877665 & 0.000000 & 1.536681 \\
\hline & tanocarbene & singlet & \\
\hline $\mathrm{C}$ & 0.119376 & -1.300055 & 0.000000 \\
\hline C & 0.000000 & 0.098492 & 0.000000 \\
\hline $\mathrm{N}$ & -0.271702 & 1.254590 & 0.000000 \\
\hline $\mathrm{H}$ & 1.185657 & -1.572754 & 0.000000 \\
\hline & tanocarbene & triplet & \\
\hline $\mathrm{C}$ & 0.275735 & -1.227963 & 0.000000 \\
\hline $\mathrm{C}$ & 0.000000 & 0.119244 & 0.000000 \\
\hline $\mathrm{N}$ & -0.191487 & 1.254368 & 0.000000 \\
\hline $\mathrm{H}$ & -0.314002 & -2.128267 & 0.000000 \\
\hline & ranomethylt & & \\
\hline C & 0.000000 & 0.000000 & -1.201225 \\
\hline C & 0.000000 & 0.000000 & 0.185363 \\
\hline $\mathrm{N}$ & 0.000000 & 0.000000 & 1.370454 \\
\hline $\mathrm{H}$ & 0.000000 & 0.945880 & -1.749006 \\
\hline $\mathrm{H}$ & 0.000000 & -0.945880 & -1.749006 \\
\hline & vetonitrile & & \\
\hline $\mathrm{C}$ & 0.000000 & 0.273873 & 0.000000 \\
\hline $\mathrm{N}$ & -0.000438 & 1.452206 & 0.000000 \\
\hline C & 0.000314 & -1.186931 & 0.000000 \\
\hline $\mathrm{H}$ & -1.024593 & -1.562585 & 0.000000 \\
\hline $\mathrm{H}$ & 0.512888 & -1.562255 & 0.887664 \\
\hline $\mathrm{H}$ & 0.512888 & -1.562255 & -0.887664 \\
\hline & Lyanocarb & ne, singlet & \\
\hline $\mathrm{C}$ & 0.000000 & 0.000000 & 0.800428 \\
\hline $\mathrm{C}$ & 0.000000 & 1.188122 & 0.062996 \\
\hline $\mathrm{N}$ & 0.000000 & 2.282781 & -0.397037 \\
\hline $\mathrm{C}$ & 0.000000 & -1.188122 & 0.062996 \\
\hline $\mathrm{N}$ & 0.000000 & -2.282781 & -0.397037 \\
\hline
\end{tabular}




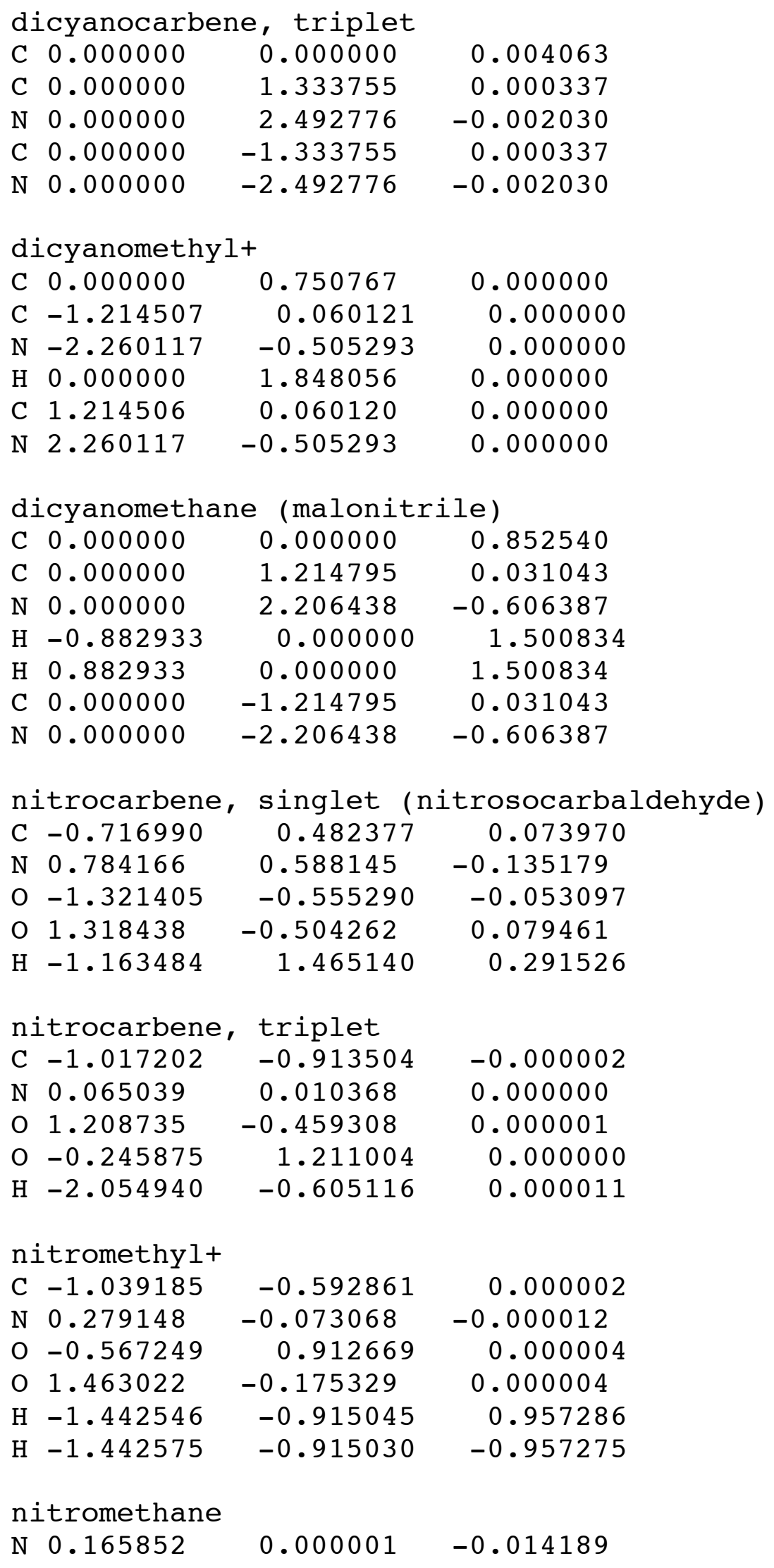




$\begin{array}{llcr}\mathrm{O} & 0.731199 & 1.103802 & 0.004135 \\ \mathrm{O} & 0.731330 & -1.103731 & 0.004136 \\ \mathrm{C}-1.319467 & -0.000060 & -0.001274 \\ \mathrm{H}-1.631786 & -0.000035 & 1.043198 \\ \mathrm{H}-1.656256 & -0.904822 & -0.501175 \\ \mathrm{H}-1.656352 & 0.904642 & -0.501221\end{array}$

dinitrocarbene, singlet

$\begin{array}{llcc}\mathrm{N} & 1.163768 & -0.106021 & 0.078099 \\ \mathrm{C} & 0.073744 & 0.060150 & 0.932104 \\ \mathrm{~N} & -1.125448 & -0.037340 & 0.048315 \\ \mathrm{O} & 1.901797 & -0.886865 & -0.480921 \\ \mathrm{O} & 1.152957 & 1.180101 & 0.121507 \\ \mathrm{O} & -1.599382 & -1.180294 & 0.078008 \\ \mathrm{O} & -1.544210 & 0.967386 & -0.528286\end{array}$

dinitrocarbene, triplet

$\begin{array}{cccc}\mathrm{N} & 1.265398 & -0.071175 & -0.083288 \\ \mathrm{C} & -0.000004 & -0.760969 & -0.000021 \\ \mathrm{~N} & -1.265394 & -0.071169 & 0.083288 \\ \mathrm{O} & 2.222649 & -0.665797 & 0.424044 \\ \mathrm{O} & 1.261784 & 1.013436 & -0.673857 \\ \mathrm{O} & -1.261784 & 1.013443 & 0.673852 \\ \mathrm{O} & -2.222649 & -0.665804 & -0.424023\end{array}$

dinitromethyl+

$\begin{array}{llcr}\mathrm{C} & -0.020072 & -0.705958 & 0.324133 \\ \mathrm{~N} & -1.254230 & -0.169621 & -0.116114 \\ \mathrm{O} & -1.052840 & 0.118080 & 1.142222 \\ \mathrm{O} & -2.092949 & 0.059843 & -0.924105 \\ \mathrm{~N} & 1.195234 & 0.077971 & -0.054236 \\ \mathrm{H} & 0.079192 & -1.760493 & 0.589380 \\ \mathrm{O} & 1.060172 & 1.300788 & -0.021637 \\ \mathrm{O} & 2.142394 & -0.648987 & -0.364196\end{array}$

dinitromethane

$\begin{array}{cccc}\mathrm{H}-0.160502 & -1.491638 & -0.909216 \\ \mathrm{C}-0.081809 & -0.898019 & -0.000581 \\ \mathrm{H}-0.160800 & -1.492823 & 0.907248 \\ \mathrm{~N}-1.205482 & 0.095745 & -0.000111 \\ \mathrm{~N} & 1.205815 & -0.169103 & 0.000101 \\ \mathrm{O} & 1.679043 & 0.116196 & -1.110394 \\ \mathrm{O} & 1.677933 & 0.115955 & 1.111125 \\ \mathrm{O}-2.328358 & -0.420799 & 0.000212 \\ \mathrm{O}-0.927389 & 1.299407 & -0.000252\end{array}$

aminocyanocarbene, singlet

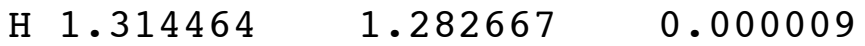




\begin{tabular}{|c|c|c|c|}
\hline $\mathrm{N}$ & 1.491487 & 0.275392 & -0.000002 \\
\hline $\mathrm{C}$ & 0.581514 & -0.670274 & -0.000002 \\
\hline & 2.470734 & 0.006761 & -0.000010 \\
\hline & -0.747017 & -0.104743 & 0.000012 \\
\hline & -1.890370 & 0.204704 & -0.000007 \\
\hline \multicolumn{4}{|c|}{ aminocyanocarbene, triplet } \\
\hline & -2.174715 & -0.237041 & -0.841504 \\
\hline & -1.606185 & -0.268852 & 0.000004 \\
\hline & -0.506172 & 0.540483 & -0.000022 \\
\hline & -2.174694 & -0.236981 & 0.841521 \\
\hline $\mathrm{C}$ & 0.842377 & 0.105156 & 0.000027 \\
\hline $\mathrm{N}$ & 1.939353 & -0.216836 & -0.000011 \\
\hline \multicolumn{4}{|c|}{ aminocyanomethyl+ } \\
\hline C & 0.000000 & 0.756867 & 0.000000 \\
\hline C & -0.628415 & -0.518874 & 0.000000 \\
\hline $\mathrm{N}$ & -1.180597 & -1.566476 & 0.000000 \\
\hline $\mathrm{H}$ & -0.602269 & 1.664559 & 0.000000 \\
\hline $\mathrm{N}$ & 1.285801 & 0.864052 & 0.000000 \\
\hline $\mathrm{H}$ & 1.737978 & 1.783453 & 0.000000 \\
\hline $\mathrm{H}$ & 1.898358 & 0.041005 & 0.000000 \\
\hline \multicolumn{4}{|c|}{ aminocyanomethane } \\
\hline $\mathrm{C}$ & -0.535877 & 0.608284 & 0.047280 \\
\hline C & 0.843394 & 0.105906 & -0.008849 \\
\hline $\mathrm{N}$ & 1.952701 & -0.292313 & -0.021251 \\
\hline $\mathrm{H}$ & -0.675779 & 1.306003 & -0.783619 \\
\hline $\mathrm{H}$ & -0.643583 & 1.187221 & 0.977292 \\
\hline $\mathrm{N}$ & -1.476613 & -0.499192 & -0.116282 \\
\hline $\mathrm{H}$ & -1.434482 & -1.109811 & 0.697364 \\
\hline $\mathrm{H}$ & -2.423878 & -0.128019 & -0.158887 \\
\hline
\end{tabular}

aminonitrocarbene, singlet
$\mathrm{N} 1.681316$
$-0.035037$
0.155349
$\begin{array}{llll}\mathrm{C} & 0.723825 & -0.787152 & -0.314477\end{array}$
$\mathrm{N}-0.539694 \quad-0.000482 \quad-0.056732$
H $1.519894 \quad 0.910643 \quad 0.518135$
$\mathrm{H} 2.637292-0.366750 \quad 0.087210$
$\begin{array}{llll}0 & -0.517157 & 1.247389 & -0.095098\end{array}$
$\begin{array}{llll}0-1.544279 & -0.693933 & 0.168999\end{array}$

aminonitrocarbene, triplet
N 1.844266
$-0.048576-0.102910$
C 0.663376
$-0.651386$
0.177794
$\mathrm{N}-0.598260$
0.000969
0.005017
H 2.178329
0.646110
0.560842
H 2.573463
$-0.656530$
$-0.457085$ 


\begin{tabular}{lcc} 
O -0.570933 & 1.244738 & -0.022263 \\
$\mathrm{O}-1.610828$ & -0.713240 & -0.038396 \\
\multicolumn{3}{c}{ aminonitrocarbenium ion } \\
$\mathrm{H}-1.650495$ & 1.034885 & -0.000177 \\
$\mathrm{~N}-1.738957$ & 0.005119 & -0.000059 \\
$\mathrm{C}-0.668802$ & -0.688295 & 0.000080 \\
$\mathrm{H}-2.670494$ & -0.424581 & -0.000043 \\
$\mathrm{~N} 0.602444$ & 0.052563 & 0.000018 \\
$\mathrm{H}-0.627303$ & -1.775380 & 0.000225 \\
$\mathrm{O} 1.607588$ & -0.674705 & -0.000073 \\
$\mathrm{O} 0.506999$ & 1.286088 & 0.000048
\end{tabular}

aminonitromethane

\begin{tabular}{|c|c|c|c|}
\hline C & -0.675032 & -0.752918 & 0.000038 \\
\hline $\mathrm{N}$ & 0.627080 & 0.058631 & 0.000004 \\
\hline 0 & 0.541500 & 1.295487 & 0.000016 \\
\hline 0 & 1.674814 & -0.600868 & -0.000029 \\
\hline $\mathrm{N}$ & -1.863178 & 0.013082 & -0.000029 \\
\hline $\mathrm{H}$ & -0.595791 & -1.381693 & 0.888158 \\
\hline $\mathrm{H}$ & -0.595782 & -1.381827 & -0.887984 \\
\hline $\mathrm{H}$ & -1.917997 & 0.611004 & -0.819861 \\
\hline $\mathrm{H}$ & -1.918051 & 0.611083 & 0.819741 \\
\hline \multicolumn{4}{|c|}{ imidazol-5-ylidene, singlet } \\
\hline $\mathrm{H}$ & -1.176374 & -1.774127 & 0.000000 \\
\hline $\mathrm{N}$ & -0.578247 & -0.955728 & 0.000000 \\
\hline $\mathrm{C}$ & -1.081510 & 0.295339 & 0.000000 \\
\hline $\mathrm{C}$ & 0.822384 & -1.052456 & 0.000000 \\
\hline $\mathrm{H}$ & -2.113068 & 0.613036 & 0.000000 \\
\hline $\mathrm{C}$ & 1.138380 & 0.306356 & 0.000000 \\
\hline $\mathrm{N}$ & 0.000000 & 1.094727 & 0.000000 \\
\hline $\mathrm{H}$ & 2.108069 & 0.784827 & 0.000000 \\
\hline $\mathrm{H}$ & -0.046420 & 2.107838 & 0.000000 \\
\hline
\end{tabular}

imidazol-5-ylidene, triplet

\begin{tabular}{|c|c|c|c|}
\hline C & -0.635633 & 0.948603 & -0.144906 \\
\hline $\mathrm{C}$ & -0.043421 & -1.205355 & -0.001496 \\
\hline $\mathrm{H}$ & -1.197470 & 1.700987 & 0.406854 \\
\hline $\mathrm{C}$ & 1.078972 & -0.546694 & 0.013916 \\
\hline $\mathrm{H}$ & 2.101421 & -0.887758 & -0.023929 \\
\hline $\mathrm{N}$ & -1.151011 & -0.391146 & -0.090241 \\
\hline $\mathrm{H}$ & -1.940951 & -0.577286 & 0.520616 \\
\hline $\mathrm{N}$ & 0.761000 & 0.833951 & 0.126602 \\
\hline $\mathrm{H}$ & 1.367572 & 1.485089 & -0.363149 \\
\hline & idazol-2 & idene-5-CH & (imidazolium+) \\
\hline & 0.000000 & 1.393514 & -1.788673 \\
\hline
\end{tabular}




\begin{tabular}{|c|c|c|c|}
\hline C & 0.000000 & 0.684743 & -0.974394 \\
\hline $\mathrm{C}$ & 0.000000 & -0.684743 & -0.974394 \\
\hline $\mathrm{N}$ & 0.000000 & 1.072375 & 0.344390 \\
\hline $\mathrm{H}$ & 0.000000 & -1.393514 & -1.788673 \\
\hline $\mathrm{N}$ & 0.000000 & -1.072375 & 0.344390 \\
\hline $\mathrm{H}$ & 0.000000 & 2.035160 & 0.676011 \\
\hline $\mathrm{H}$ & 0.000000 & -2.035160 & 0.676011 \\
\hline $\mathrm{C}$ & 0.000000 & 0.000000 & 1.145212 \\
\hline $\mathrm{H}$ & 0.000000 & 0.000000 & 2.225317 \\
\hline \multicolumn{4}{|c|}{ imidazol-5-ylidene, 5-CH2 } \\
\hline $\mathrm{C}$ & -0.750721 & -0.935822 & 0.166223 \\
\hline $\mathrm{N}$ & -1.085526 & 0.372294 & 0.042285 \\
\hline $\mathrm{H}$ & -2.052206 & 0.679061 & 0.024974 \\
\hline $\mathrm{H}$ & -1.445017 & -1.693009 & -0.190623 \\
\hline $\mathrm{N}$ & 0.616426 & -0.975950 & -0.238086 \\
\hline $\mathrm{H}$ & 1.130958 & -1.736576 & 0.200024 \\
\hline C & -0.032904 & 1.204753 & -0.200945 \\
\hline $\mathrm{H}$ & -0.125294 & 2.247975 & 0.081788 \\
\hline C & 1.163526 & 0.354351 & 0.121834 \\
\hline $\mathrm{H}$ & 2.053999 & 0.582515 & -0.470693 \\
\hline $\mathrm{H}$ & 1.441854 & 0.405927 & 1.202471 \\
\hline
\end{tabular}


S125 
Table S6. G3MP2 enthalpies of hydrogenation and singlet-triplet enthalpy gaps for a large range of singlet carbene types. HIA and $\Delta H_{\mathrm{ACID}}$ for the corresponding carbenium ions, $298 \mathrm{~K}, \mathrm{kcal} / \mathrm{mol}^{\mathrm{a}}$

Carbene

$\Delta \underline{H}_{\text {HYDROG(singlet) }}$

$\Delta \underline{H}_{\mathrm{ST}}$

HIA

$\Delta \underline{H}_{\mathrm{ACID}}$

$\underline{\text { Type 1. alkyl, alkenyl and alkynyl carbenes }}{ }^{\mathrm{b}}$

$\begin{array}{lllll}\mathrm{CH}_{2} & -118.1 & 9.4 & -314.5 & 205.2 \\ \mathrm{CH}_{3} \mathrm{CH} & -106.0 & 2.9 & -269.3 & 235.6 \\ \left(\mathrm{CH}_{3}\right)_{2} \mathrm{CH}-\mathrm{CH} & -104.9 & 0.6 & \text { NA } & \text { NA } \\ \left(\mathrm{CH}_{3}\right)_{2} \mathrm{C} & -97.1 & -1.9 & -250.2 & 245.9 \\ \mathrm{CH}_{2}=\mathrm{CH}-\mathrm{CH} & -100.1 & 8.2 & -256.3 & 242.8 \\ \mathrm{CH} \equiv \mathrm{C}-\mathrm{CH} & -96.1 & 11.4 & -272.7 & 222.3 \\ \text { diethynylcarbene } & -78.6 & 12.6 & -252.5 & 225.1 \\ \text { cyclopropylidene } & -97.1 & -16.7 & -245.3 & 250.7 \\ \text { cyclobutylidene } & -91.6 & -10.4 & -239.3 & 251.3 \\ \text { cyclopentylidene } & -90.5 & -9.9 & -243.6 & 245.8 \\ \text { cyclopropenylidene } & -49.0 & -71.7 & -222.5 & 225.4 \\ \text { cyclopropylcarbinylidene } & -95.1 & -6.5 & -241.2 & 252.9 \\ \text { cyclobut-1-en-3-ylidene } & -72.5 & -24.2 & -239.6 & 231.8 \\ \text { cyclopent-1-en-1-ylidene } & -86.6 & -7.6 & -226.3 & 259.2 \\ \text { 2,4-cyclohexadienylidene } & -83.4 & 3.2 & -215.0 & 267.3 \\ \text { 2,5-cyclohexadienylidene } & -85.7 & 3.2 & -215.1 & 269.5 \\ \text { 6,6-dimethylcyclohexa-2,4-dienylidene } & -87.4 & 5.3 & -214.6 & 271.9 \\ \text { spiro[2.5]octa-4,6-dien-8-ylidene } & -71.5 & -7.4 & -195.6 & 274.8 \\ \text { spiro[2.5]octa-4,6-dien-6-ylidene } & -81.1 & 0.8 & -196.5 & 283.6\end{array}$

Type 2. aryl carbenes ${ }^{\mathrm{c}}$

$\mathrm{PhCH}$

3-oxyphenyl ${ }^{-}$

3-aminophenyl

3-hydroxyphenyl

3-sulfhydrylphenyl

3-methylphenyl

3-fluorophenyl

3-chlorophenyl

3-formylphenyl

3-trifluoromethylphenyl

3-cyanophenyl

3-nitrophenyl

4-oxyphenyl ${ }^{-}$

4-aminophenyl

4-hydroxyphenyl

4-sulfhydrylphenyl

4-methylphenyl

4-fluoromethylphenyl
$-99.4$

$-87.2$

$-98.9$

$-99.1$

$-99.7$

$-99.6$

$-100.7$

$-100.8$

$-100.6$

$-101.4$

$-102.1$

$-102.4$

$-64.5$

$-92.5$

$-95.5$

$-97.8$

$-98.0$

$-99.1$
2.13

$-8.2$

1.53

1.70

1.63

1.63

2.72

2.47

2.24

3.02

3.05

3.38

$-24.3$

$-4.05$

$-1.70$

$-0.04$

0.78

1.49
$-240.5$

$-127.1$

$-231.5$

$-237.8$

$-239.4$

$-236.0$

$-244.6$

$-243.9$

$-245.6$

$-247.9$

$-252.1$

$-252.7$

$-87.5$

$-210.6$

$-224.8$

$-227.1$

0231.3

$-236.6$
259.9

359.0

266.4

260.3

259.2

262.6

255.1

255.8

254.0

252.5

249.0

248.6

376.0

280.9

269.6

269.7

265.6

261.5 
Table S6. G3MP2 enthalpies of hydrogenation and singlet-triplet enthalpy gaps for a large range of singlet carbene types. HIA and $\Delta H_{\mathrm{ACID}}$ for the corresponding carbenium ions, $298 \mathrm{~K}, \mathrm{kcal} / \mathrm{mol}^{\mathrm{a}}$

$\begin{array}{lllll}\text { Carbene } & \Delta H_{\underline{\text { HYROG(singlet) }}} & \Delta H_{\text {ST }} & \text { HIA } & \underline{H_{\text {ACID }}} \\ \text { 4-fluorophenyl } & & & & \\ \text { 4-chlorophenyl } & -98.2 & 0.22 & -237.5 & 259.6 \\ \text { 4-formylphenyl } & -99.3 & 1.40 & -237.4 & 260.5 \\ \text { 4-trifluoromethylphenyl } & -101.9 & 4.52 & -246.6 & 254.3 \\ \text { 4-cyanophenyl } & -192.6 & 3.79 & -249.0 & 252.5 \\ \text { 4-nitrophenyl } & -102.4 & 4.39 & -251.2 & 250.2 \\ \mathrm{PhCCH}_{3} & -103.0 & 4.98 & -253.6 & 248.4 \\ \mathrm{Ph}_{2} \mathrm{C} & -93.2 & 0.8 & -229.3 & 264.9 \\ \text { cycloheptatrienylcarbene }^{+} & -86.4 & -2.6 & -217.4 & 268.0 \\ \text { cyclopentadienylcarbene }^{-} & -108.2 & 9.2 & -369.9 & 137.2 \\ \text { cyclopropenylcarbene }^{+} & -59.8 & -26.4 & -70.0 & 388.7\end{array}$

Type 3, acyclic heteroatom and cyano carbenes ${ }^{\mathrm{c}}$

\begin{tabular}{|c|c|c|c|c|}
\hline $\mathrm{NH}_{2} \mathrm{CH}$ & -60.0 & -34.5 & -217.1 & 241.9 \\
\hline$\left(\mathrm{NH}_{2}\right)_{2} \mathrm{C}$ & -35.2 & -57.4 & -184.5 & 249.6 \\
\hline $\mathrm{HOCH}$ (anti) & -71.8 & -25.6 & -249.9 & 220.9 \\
\hline HOCH (syn) & -76.4 & -21.0 & -249.9 & 225.5 \\
\hline $\mathrm{CH}_{3} \mathrm{OCH}$ & -70.2 & -28.1 & -238.9 & 232.2 \\
\hline $\mathrm{CH}_{3} \mathrm{OCCH}_{3}$ & -64.6 & -32.7 & -221.1 & 242.5 \\
\hline$\left(\mathrm{CH}_{3} \mathrm{O}\right)_{2} \mathrm{C}$ & -40.8 & -59.8 & -206.2 & 235.6 \\
\hline $\mathrm{FCH}$ & -89.6 & -14.6 & -289.5 & 199.1 \\
\hline $\mathrm{F}_{2} \mathrm{C}$ & -59.5 & -56.3 & -282.7 & 175.7 \\
\hline $\mathrm{SiH}_{3} \mathrm{CH}$ & -117.4 & 18.1 & $-241.4^{\mathrm{d}}$ & $274.9^{d}$ \\
\hline$\left(\mathrm{SiH}_{3}\right)_{2} \mathrm{C}$ & -113.7 & 22.0 & $-226.4^{\mathrm{e}}$ & $281.7^{\mathrm{e}}$ \\
\hline $\mathrm{PH}_{2} \mathrm{CH}$ & -83.9 & -13.4 & -249.3 & 233.5 \\
\hline HSCH (anti) & -75.4 & -20.6 & -247.7 & 226.7 \\
\hline HSCH (syn) & -76.6 & -19.4 & -247.7 & 227.9 \\
\hline $\mathrm{CH}_{3} \mathrm{SCH}$ & -69.7 & -24.9 & -233.9 & 234.7 \\
\hline $\mathrm{ClCH}$ & -93.7 & -6.9 & -279.7 & 212.9 \\
\hline $\mathrm{Cl}_{2} \mathrm{C}$ & -74.4 & -21.8 & -266.2 & 207.1 \\
\hline$\left(\mathrm{NH}_{2}\right)_{2} \mathrm{CCH}^{+}$ & -120.1 & 12.1 & -428.3 & 90.7 \\
\hline cyanocarbene & -106.6 & 9.9 & -313.5 & 192.0 \\
\hline dicyanocarbene & -94.5 & 11.8 & -315.3 & 178.2 \\
\hline nitrocarbene & $\mathrm{NA}^{\mathrm{f}}$ & $\mathrm{NA}^{\mathrm{f}}$ & -293.6 & $\mathrm{NA}^{\mathrm{f}}$ \\
\hline dinitrocarbene & -93.1 & -19.7 & -309.0 & 183.0 \\
\hline aminocyanocarbene & -52.8 & -32.4 & -234.2 & 217.6 \\
\hline aminonitrocarbene & -58.0 & -41.5 & -245.8 & 211.2 \\
\hline $\mathrm{O}=\mathrm{NCH}$ & -21.7 & -60.4 & -255.9 & 164.8 \\
\hline $\mathrm{HN}=\mathrm{C}$ & -22.9 & -106.8 & -238.8 & 183.0 \\
\hline $\mathrm{O}=\mathrm{C}$ & 2.2 & -138.1 & -256.5 & 140.3 \\
\hline
\end{tabular}


Table S6. G3MP2 enthalpies of hydrogenation and singlet-triplet enthalpy gaps for a large range of singlet carbene types. HIA and $\Delta H_{\mathrm{ACID}}$ for the corresponding carbenium ions, $298 \mathrm{~K}, \mathrm{kcal} / \mathrm{mol}^{\mathrm{a}}{ }^{\mathrm{a}}$

Carbene

$\Delta H_{\text {HYDROG(singlet) }}$

$\Delta H_{\underline{S T}}$

HIA

$\Delta H_{\underline{A C I D}}$

Type 4. Five-membered cyclic 1,3-diheteroatom carbenes ${ }^{\mathrm{g}}$

$\begin{array}{lllll}\text { imidazol-2-ylidene } & -15.0 & -85.7 & -163.9 & 250.1 \\ \text { 4,5-dihydroimidazol-2-ylidene } & -30.7 & -72.3 & -176.9 & 252.8 \\ N, N \text { '-dimethylimidazol-2-ylidene } & -14.9 & -87.6 & -155.5 & 258.4 \\ \text { 4,5-dimethylimidazol-2-ylidene } & -16.7 & -83.6 & -158.9 & 257.6 \\ \text { 4,5,N,N'-tetramethylimidazol-2-ylidene -14.5 } & -86.3 & -148.4 & 265.0 \\ \text { imidazol-5-ylidene } & -3.8 & 65.3 & -133.3 & 269.5 \\ \text { 1,3-dioxol-2-ylidene } & -33.7 & -86.6 & -212.0 & 220.6 \\ \text { 1,3-dioxolane-2-ylidene } & -38.1 & -72.7 & -208.5 & 228.5 \\ \text { oxazol-2-ylidene } & -26.3 & -86.8 & -188.0 & 237.3 \\ \text { 4,5-dihydrooxazol-2-ylidene } & -36.2 & -70.2 & -192.5 & 242.7 \\ \text { 4,5-difluoroimidazol-2-ylidene } & -24.5 & -65.8 & -184.7 & 238.7 \\ \text { 4,5,N,N'-tetrasilylimidazol-2-ylidene } & -16.9 & -83.0 & -157.6 & 258.2 \\ \text { 1,3-diphosphole-2-ylidene } & -70.0 & -26.1 & -226.0 & 243.0 \\ \text { 1,3-diphospholan-2-ylidene } & -74.9 & -22.0 & -228.9 & 245.0 \\ \text { 1,3-dithiol-2-ylidene } & -35.6 & -56.9 & -200.3 & 234.3 \\ \text { 1,3-dithiolan-2-ylidene } & -46.9 & -47.2 & -210.6 & 235.3\end{array}$

Type 5. small-ring aza- and diazacarbenes ${ }^{\mathrm{g}}$

$\begin{array}{lllll}\text { diazirene-3-ylidene } & =61.6 & -30.5 & -288.2 & 172.4 \\ \text { diazirane-3-ylidene } & -64.5 & -55.0 & -260.5 & 203.0 \\ \text { 1,3-diazetidin-2-ylidene } & -38.7 & -62.8 & -191.3 & 246.4 \\ \text { azet-2-ylidene } & -71.3 & -39.3 & -241.9 & 228.4 \\ \text { azetidin-2-ylidene } & -53.8 & -46.9 & -203.2 & 249.6\end{array}$

${ }^{\mathrm{a}}$ Hydrogenation of the carbene occurs with the addition of two hydrogen atoms to the carbenic carbon. A negative value for $\Delta H_{\mathrm{ST}}$ indicates the singlet is more stable. HIA $=$ the reaction enthalpy for addition of hydride to the carbenium ion. $\Delta H_{\mathrm{ACID}}$ is the reaction enthalpy for loss of a proton from the $\alpha$-carbon of the carbenium ion. ${ }^{\mathrm{b}}$ See also Keeffe, J. R.; More O'Ferrall, R. A. ARKIVOC 2008, $183 .{ }^{\mathrm{c}} \Delta H_{\mathrm{ST}}$ and $\Delta H_{\text {HYDROG }}$ values for a number of examples of Types 1, 2 and 3 carbenes are given in Gronert, S.; Keeffe, J. R.; More O'Ferrall. R. A. J. Org. Chem. 2009, 74. 5250. ${ }^{\mathrm{d}}$ Protonation of singlet silylcarbene leads to rearrangement resulting in the methylsilylium ion. The listed values are obtained using the latter species. ${ }^{~}$ Protonation of singlet disilylcarbene leads to rearrangement resulting first in silylmethylsilylium ion, $\mathrm{H}_{3} \mathrm{Si}_{-} \mathrm{CH}_{2}-\mathrm{SIH}_{2}{ }^{+}$, then the symmetrical $\left(\mathrm{C}_{2 \mathrm{v}}\right)$ 1,3-bridged $\mu$-hydridodisilylmethane cation, $10.6 \mathrm{kcal} / \mathrm{mol}$ more stable. The listed values are obtained using the latter structure. ${ }^{\mathrm{f}}$ Singlet nitrocarbene shows a partially oxygen-bridged structure at $\mathrm{HF} / 3-21 \mathrm{G}$, and a $\mathrm{C}_{\mathrm{s}}$ structure at $\mathrm{HF} / 6-31 \mathrm{G}^{*}$ with the nitro group perpendicular to the $\mathrm{CH}$ moiety. However it rearranges at MP2/6-31G* by a 1,2-oxygen migration from nitrogen to carbon yielding "nitrosocarbaldehyde". See also Tao, Y.; Ding, Y.; Li, Z.; Huang, X.; Sun, C.-C. J. Phys. Chem. A 2001, 105, 3388. ${ }^{\mathrm{g}}$ Where cis and trans isomers exist, the more stable of the two is used for the $\Delta H$ calculations. 